\title{
Cobalt-Catalyzed Chemoselective Transfer Hydrogenative Cyclization Cascade of Enone-Tethered Aldehydes
}

\section{Supporting Information}

Shuang-Shuang Ma, a,b Biao-Ling Jiang, ${ }^{a}$ Zheng-Kun Yu, ${ }^{c}$ Suo-Jiang Zhang, and Bao-Hua Xu ${ }^{a, b, c *}$

a Beijing Key Laboratory of Ionic Liquids Clean Process, Key Laboratory of Green Process and Engineering, State Key Laboratory of Multiphase Complex Systems, Institution of Process Engineering, Chinese Academy of Sciences, Beijing 100190, China

${ }^{b}$ College of Chemistry and Chemical Engineering, University of Chinese Academy of Sciences, Beijing, 100049, China

${ }^{c}$ Innovation Academy for Green Manufacture, Chinese Academy of Sciences, Beijing, 100190, China

*Corresponding Authors: Bao-Hua Xu,Email: bhxu@ipe.ac.cn

\section{Table of Contents}

Supporting Information .1

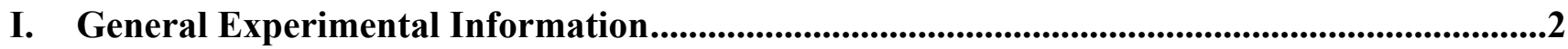

II. Synthesis of the Starting Substrates .......................................................................................

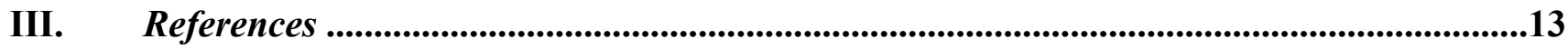

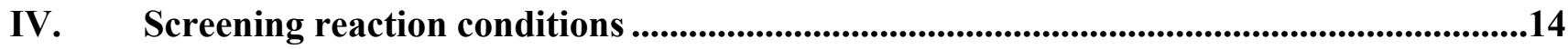

V. Cobalt-Catalyzed Cyclization Cascade of Enone-Tethered Aldehydes .....................................15

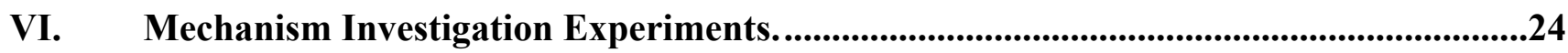

VII. Cobalt-Catalyzed Reductive Enone-Tethered Aldehydes Cyclization with TEMPO....30

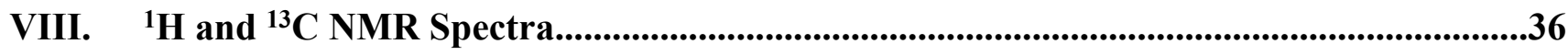




\section{General Experimental Information}

All reactions (except for the preparation of starting substrates) were prepared and sealed using a glass flask in a glove box filled with argon. Then the reactions were carried out on a multi-zone reaction platform which was purchased from Tokyo Rikakikai Co., LTD (CCX-1102). Solvents were distilled from sodium and benzophenone ketyl immediately prior to use. ESI-MS analysis was performed on a Bruker time of flight mass spectrometer microTOF-Q II using an electrospray ionization (ESI) source. NMR spectra were recorded on a Bruker ASCEND spectrometer $\left({ }^{1} \mathrm{H}, 600 \mathrm{MHz} ;{ }^{13} \mathrm{C}\left\{{ }^{1} \mathrm{H}\right\}, 151 \mathrm{MHz}\right) .{ }^{1} \mathrm{H}$ NMR and ${ }^{13} \mathrm{C}$ NMR, chemical shift $\delta$ is given relative to TMS and referenced to the solvent signal. Chemical shifts were reported in ppm with the internal TMS signal at $0.0 \mathrm{ppm}$ as a standard. The spectra are interpreted as: $\mathrm{s}=$ singlet, $\mathrm{d}=$ doublet, $\mathrm{t}=$ triplet, $\mathrm{m}=$ multiplet, $\mathrm{dd}=$ doublet of doublets, $\mathrm{dt}=$ doublet of triplets, ddd $=$ doublet of doublet of doublets, coupling constants $(J)$ are reported in $\mathrm{Hz}$ and relative integrations are reported. Column chromatography was performed using silica gel. Analytical TLC was done using pre-coated silica gel 60 F254 plates. GC-MS was performed using GCMS-QP2020 with Rtx$5 \mathrm{MS}(30 \mathrm{~m} \times 0.25 \mathrm{~mm})$ column.

\section{Synthesis of the Starting Substrates}

The general procedure-1: Synthesis of enone-tethered aldehydes (1a-z).$^{1-8}$

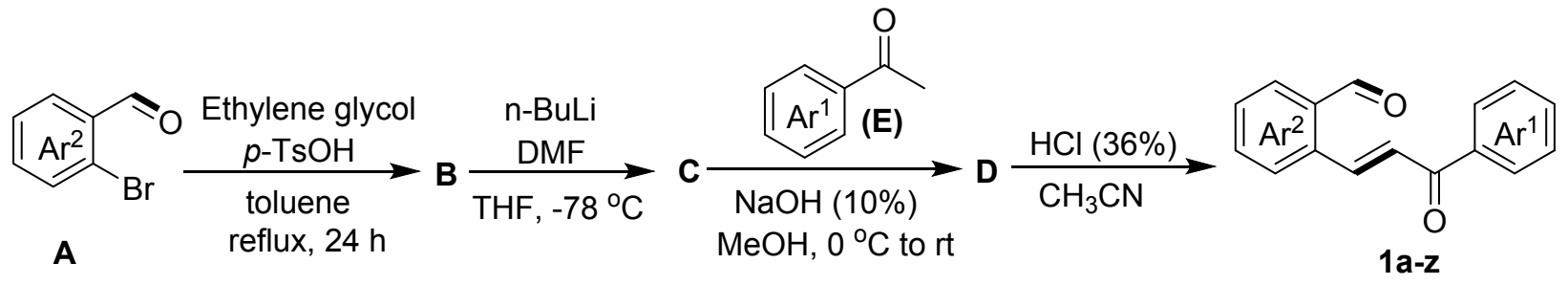

The 2-bromobenzaldehyde A (15 mmol), ethylene glycol $(75 \mathrm{mmol})$, and $p$-toluenesulfonic acid (0.45 mmol) were dissolved in $30 \mathrm{ml}$ of toluene. The mixture was refluxed in an oil bath for $24 \mathrm{~h}$ and then transferred to a separating funnel. Water $(50 \mathrm{~mL})$ was added and followed by extraction with ethyl acetate $(2 \times 50 \mathrm{~mL})$. The organic layer was dried over anhydrous sodium sulfate. The solvent was evaporated in vacuo and purified by column chromatography to afford 2-bromoacetals $\mathbf{B}^{\mathbf{1 , 2}}$ Then the obtained acetals B $(10 \mathrm{mmol})$ was dissolved in $30 \mathrm{ml}$ of dry THF and cooled to $-78{ }^{\circ} \mathrm{C}$ in a low temperature bath under argon. Then a solution of n-butyl lithium ( $15 \mathrm{mmol}, 2.5 \mathrm{M}$ solution in hexane) was added drop wise and stirred for $40 \mathrm{~min}$ at the same temperature. Immediately after DMF (20 mmol) was added drop wise, the 
reaction mixture was warmed up to $0{ }^{\circ} \mathrm{C}$ and stirrer in a low temperature bath for $6 \mathrm{~h}$. The reaction was quenched with a saturated solution of ammonium chloride and $20 \mathrm{~mL}$ of water, the mixture was extracted with ethyl acetate $(3 \times 15 \mathrm{~mL})$. The organic phase was subsequently washed with brine $(1 \times 10 \mathrm{~mL})$ and dried over anhydrous sodium sulfate. The solvent was evaporated in vacuo and the crude was purified by flash column chromatography to afford $\mathbf{C} .^{3-5}$ the $\mathbf{E}(1 \mathrm{mmol})$ was added in $3 \mathrm{~mL}$ ethanol, then the mixture was cooled to $0{ }^{\circ} \mathrm{C}$ in a low temperature bath. A solution of an aqueous solution of $10 \% \mathrm{NaOH}(2 \mathrm{~mL})$ was added drop wise at $0{ }^{\circ} \mathrm{C}$. After stirring for $15 \mathrm{~min}$, the aromatic aldehyde $\mathbf{C}(1 \mathrm{mmol})$ added and stirred at $0{ }^{\circ} \mathrm{C}$ for additional $30 \mathrm{~min}$. The mixture is then allowed to reach room temperature and stirrer for $6 \mathrm{~h}$. After completion, the reaction mixture was transferred into a separating funnel with $5 \mathrm{~mL}$ water and the mixture was extracted with ethyl acetate $(3 \times 10 \mathrm{~mL})$ and the organic phase was subsequently washed with brine $(1 \times 5 \mathrm{~mL})$, followed by drying over anhydrous sodium sulfate. The solvent was evaporated in vacuo and the crude product was purified by column chromatography to afford the desired acetals $\mathbf{D} .{ }^{6}$ To a solution of $\mathbf{D}(1.0 \mathrm{mmol})$ in acetonitrile $(3 \mathrm{~mL})$ was added drop wise aqueous $\mathrm{HCl}(1$ $\mathrm{mL}, 36 \%$ ) at $0{ }^{\circ} \mathrm{C}$. The reaction mixture was allowed to stir at the same temperature for $10 \mathrm{~min}$, and then neutralized with a saturated solution of $\mathrm{NaHCO}_{3}$. the mixture was extracted with ethyl acetate $(3 \times 10$ $\mathrm{mL})$ and the organic phase was subsequently washed with brine $(1 \times 5 \mathrm{~mL})$, followed by drying over anhydrous sodium sulfate. The solvent was evaporated in vacuo and the crude product was purified by column chromatography to afford the desired enone-tethered aldehydes 1a-z. ${ }^{7,8}$

\section{(2-bromophenyl)-1,3-dioxolane (representative data of B)}<smiles>Brc1ccccc1C1OCCO1</smiles>

Yield 3.043 g, 89\%; (petroleum ether/ethyl acetate $=5: 1)$; colourless oil; ${ }^{1}$ H NMR $(600$ $\left.\mathrm{MHz}, \mathrm{CDCl}_{3}, 298 \mathrm{~K}\right): \delta=7.61\left(\mathrm{dd},{ }^{3} J_{\mathrm{HH}}=7.8 \mathrm{~Hz},{ }^{4} J_{\mathrm{HH}}=1.8 \mathrm{~Hz}, 1 \mathrm{H}, \mathrm{Ph}\right), 7.56\left(\mathrm{dd},{ }^{3} J_{\mathrm{HH}}\right.$ $\left.=7.8 \mathrm{~Hz},{ }^{4} J_{\mathrm{HH}}=1.2 \mathrm{~Hz}, 1 \mathrm{H}, \mathrm{Ph}\right), 7.33\left(\mathrm{td},{ }^{3} J_{\mathrm{HH}}=7.2 \mathrm{~Hz},{ }^{4} J_{\mathrm{HH}}=0.6 \mathrm{~Hz}, 1 \mathrm{H}, \mathrm{Ph}\right), 7.21$ $\left(\mathrm{td},{ }^{3} J_{\mathrm{HH}}=7.8 \mathrm{~Hz},{ }^{4} J_{\mathrm{HH}}=1.8 \mathrm{~Hz}, 1 \mathrm{H}, \mathrm{Ph}\right), 6.10(\mathrm{~s}, 1 \mathrm{H}, \mathrm{CH}), 4.14\left(\mathrm{~m}, 2 \mathrm{H}, \mathrm{CH}_{2}\right), 4.05\left(\mathrm{~m}, 2 \mathrm{H}, \mathrm{CH}_{2}\right) \cdot{ }^{13} \mathbf{C}$ NMR (151 MHz, $\left.\mathrm{CDCl}_{3}, 298 \mathrm{~K}\right): \delta=136.6,132.9,130.6,127.8,127.4,122.9,102.6(\mathrm{CH}), 65.5\left(\mathrm{CH}_{2}\right)$. GC-MS ( $\mathrm{m} / \mathrm{z})$ : Calcd. 228; Found 228.

(1,3-dioxolan-2-yl)benzaldehyde (representative data of C)<smiles>O=Cc1ccccc1C1OCCO1</smiles>

Yield 1.638 g, 92\%; (petroleum ether/ethyl acetate = 10:1); colourless oil; ${ }^{1} \mathbf{H}$ NMR $(600$ $\left.\mathrm{MHz}, \mathrm{CDCl}_{3}, 298 \mathrm{~K}\right): \delta=10.36(\mathrm{~s}, 1 \mathrm{H}, \mathrm{CHO}), 7.88\left(\mathrm{dd},{ }^{3} J_{\mathrm{HH}}=7.8 \mathrm{~Hz},{ }^{4} J_{\mathrm{HH}}=1.2 \mathrm{~Hz}\right.$, $1 \mathrm{H}, \mathrm{Ph}), 7.68\left(\mathrm{~d},{ }^{3} J_{\mathrm{HH}}=7.8 \mathrm{~Hz}, 1 \mathrm{H}, \mathrm{Ph}\right), 7.55\left(\mathrm{td},{ }^{3} J_{\mathrm{HH}}=7.8 \mathrm{~Hz},{ }^{4} J_{\mathrm{HH}}=1.2 \mathrm{~Hz}, 1 \mathrm{H}, \mathrm{Ph}\right)$, 
$7.47\left(\mathrm{td},{ }^{3} J_{\mathrm{HH}}=7.8 \mathrm{~Hz},{ }^{4} J_{\mathrm{HH}}=0.6 \mathrm{~Hz}, 1 \mathrm{H}, \mathrm{Ph}\right), 6.34(\mathrm{~s}, 1 \mathrm{H}, \mathrm{CH}), 4.05\left(\mathrm{~m}, 4 \mathrm{H}, \mathrm{CH}_{2}\right) .{ }^{13} \mathbf{C}$ NMR $(151$ $\mathrm{MHz}, \mathrm{CDCl} 3,298 \mathrm{~K}): \delta=191.7(\mathrm{CHO}), 139.0,134.4,133.5,129.9,129.4,126.9,101.0(\mathrm{CH}), 65.3$ $\left(\mathrm{CH}_{2}\right)$. GC-MS (m/z): Calcd. 178; Found 178.

(E)-3-(2-(1,3-dioxolan-2-yl)phenyl)-1-phenylprop-2-en-1-one (representative data of D)<smiles>O=C(/C=C/c1ccccc1C1OCCO1)c1ccccc1</smiles>
Yield 3.043 g, 89\%; (petroleum ether/ethyl acetate = 5:1); colourless oil; ${ }^{1} \mathbf{H}$ NMR $\left(600 \mathrm{MHz}, \mathrm{CDCl}_{3}, 298 \mathrm{~K}\right): \delta=8.28\left(\mathrm{~d},{ }^{3} \mathrm{~J}_{\mathrm{HH}}=15.6 \mathrm{~Hz}, 1 \mathrm{H}\right), 8.03\left(\mathrm{~d},{ }^{3} J_{\mathrm{HH}}\right.$ $=7.2 \mathrm{~Hz}, 2 \mathrm{H}), 7.74(\mathrm{~m}, 1 \mathrm{H}), 7.65(\mathrm{~m}, 1 \mathrm{H}), 7.58\left(\mathrm{t},{ }^{3} J_{\mathrm{HH}}=7.8 \mathrm{~Hz}, 1 \mathrm{H}\right), 7.50(\mathrm{t}$, $\left.{ }^{3} J_{\mathrm{HH}}=7.8 \mathrm{~Hz}, 2 \mathrm{H}\right), 7.46\left(\mathrm{~d},{ }^{3} J_{\mathrm{HH}}=15.6 \mathrm{~Hz}, 1 \mathrm{H}\right), 7.42(\mathrm{~m}, 2 \mathrm{H}), 6.05(\mathrm{~s}, 1 \mathrm{H}, \mathrm{CH})$, $4.16\left(\mathrm{~m}, 2 \mathrm{H}, \mathrm{CH}_{2}\right), 4.06\left(\mathrm{~m}, 2 \mathrm{H}, \mathrm{CH}_{2}\right) .{ }^{13} \mathbf{C} \mathbf{N M R}\left(151 \mathrm{MHz}, \mathrm{CDCl}_{3}, 298 \mathrm{~K}\right): \delta=190.6(\mathrm{C}=\mathrm{O}), 142.0$, 138.1, 136.5, 134.3, 132.8, 130.0, 129.5, 128.6 (4C), 127.2, 127.1, 124.4, 102.1 (CH), 65.5 (2C). HRMS (ESI) $m / z$ calcd for $\mathrm{C}_{18} \mathrm{H}_{16} \mathrm{NaO}_{3}{ }^{+}(\mathrm{M}+\mathrm{Na})^{+}, 303.0992$; found: 303.0997 .

The general procedure-2: Synthesis of 2-(3-oxo-3-phenylpropyl)benzaldehyde (9a). ${ }^{9}$

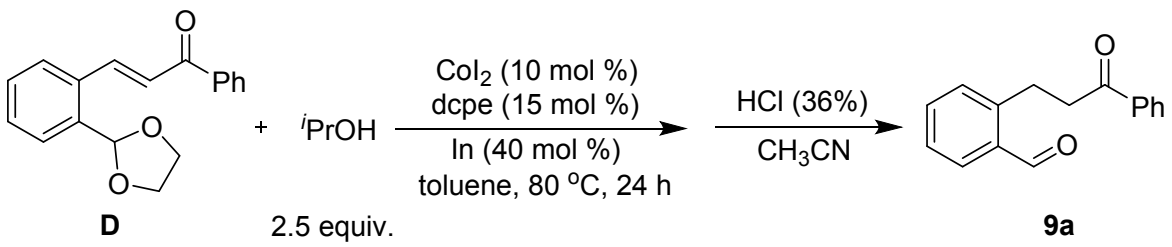

In an argon-filled glove box, To a dry flask containing a toluene $(1 \mathrm{~mL})$ solution of $\mathrm{CoI}_{2}(0.02 \mathrm{mmol}$, $10 \mathrm{~mol} \%)$, In (0.08 mmol, $40 \mathrm{~mol} \%)$, dcpe ( $0.03 \mathrm{mmol}, 15 \mathrm{~mol} \%)$ and ${ }^{i} \mathrm{PrOH}(0.5 \mathrm{mmol}, 2.5$ equiv. $)$ were sequentially added. The mixture was then stirred at $80^{\circ} \mathrm{C}$ in an oil bath for $24 \mathrm{~h}$. At the end of the reaction, the mixture was cooled to room temperature and quenched with saturated ammonium chloride solution $(5 \mathrm{~mL})$. The combined mixture was extracted with EtOAc $(3 \times 10 \mathrm{~mL})$. The organic extracts were collected and concentrated after filtering, then the crude product in acetonitrile $(3 \mathrm{~mL})$ was added drop wise aqueous $\mathrm{HCl}(1 \mathrm{~mL}, 36 \%)$ at $0{ }^{\circ} \mathrm{C}$. The reaction mixture was allowed to stir at the same temperature for $10 \mathrm{~min}$, and then neutralized with a saturated solution of $\mathrm{NaHCO}_{3}$. the mixture was extracted with ethyl acetate $(3 \times 10 \mathrm{~mL})$ and the organic phase was dried over anhydrous $\mathrm{Na}_{2} \mathrm{SO}_{4}$. The solvent was evaporated in vacuo and the crude product was purified by column chromatography to afford 9a in $89 \%$.

\section{2-(3-oxo-3-phenylpropyl)benzaldehyde (9a)}<smiles>O=Cc1ccccc1CCC(=O)c1ccccc1</smiles>

$9 a$
Yield 0.042 g, 89\%; (petroleum ether/ethyl acetate $=5: 1$ ); light yellow solid; ${ }^{1} \mathbf{H}$ NMR $\left(600 \mathrm{MHz}, \mathrm{CDCl}_{3}, 298 \mathrm{~K}\right): \delta=10.25(\mathrm{~s}, 1 \mathrm{H}, \mathrm{CHO}), 7.97\left(\mathrm{dd},{ }^{3} J_{\mathrm{HH}}=8.4 \mathrm{~Hz}\right.$, 
$\left.{ }^{4} J_{\mathrm{HH}}=1.2 \mathrm{~Hz}, 2 \mathrm{H}, \mathrm{Ph}\right), 7.82\left(\mathrm{dd},{ }^{3} J_{\mathrm{HH}}=7.8 \mathrm{~Hz},{ }^{4} J_{\mathrm{HH}}=1.2 \mathrm{~Hz}, 1 \mathrm{H}, \mathrm{Ph}\right), 7.53(\mathrm{~m}, 2 \mathrm{H}, \mathrm{Ph}), 7.45\left(\mathrm{t},{ }^{3} J_{\mathrm{HH}}\right.$ $=7.8 \mathrm{~Hz}, 2 \mathrm{H}, \mathrm{Ph}), 7.40\left(\mathrm{t},{ }^{3} J_{\mathrm{HH}}=8.4 \mathrm{~Hz}, 2 \mathrm{H}, \mathrm{Ph}\right), 3.47\left(\mathrm{t},{ }^{3} J_{\mathrm{HH}}=7.8 \mathrm{~Hz}, 2 \mathrm{H}, \mathrm{CH}_{2}\right), 3.32\left(\mathrm{t},{ }^{3} J_{\mathrm{HH}}=7.8\right.$ $\left.\mathrm{Hz}, 2 \mathrm{H}, \mathrm{CH}_{2}\right) .{ }^{13} \mathbf{C}$ NMR $\left(151 \mathrm{MHz}, \mathrm{CDCl}_{3}, 298 \mathrm{~K}\right): \delta=199.1(\mathrm{CHO}), 193.0(\mathrm{C}=\mathrm{O}), 143.9,136.9,134.1$ (2C), 133.8, 133.2, 131.8, 128.7 (2C), $128.2(2 \mathrm{C}), 127.1,40.3\left(\mathrm{CH}_{2}\right), 27.7\left(\mathrm{CH}_{2}\right)$. HRMS (ESI) $m / z$ calcd for $\mathrm{C}_{16} \mathrm{H}_{14} \mathrm{NaO}_{2}{ }^{+}(\mathrm{M}+\mathrm{Na})^{+}, 261.0886$; found: 261.0871 .

(E)-2-(3-oxo-3-phenylprop-1-en-1-yl)benzaldehyde (1a)

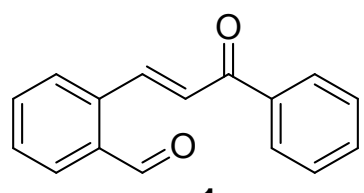

1a

Yield 0.200 g, 85\%; (petroleum ether/ethyl acetate $=5: 1$ ); light yellow solid; ${ }^{1} \mathbf{H}$

NMR (600 MHz, $\left.\mathrm{CDCl}_{3}, 298 \mathrm{~K}\right): \delta=10.34(\mathrm{~s}, 1 \mathrm{H}, \mathrm{CHO}), 8.56\left(\mathrm{~d},{ }^{3} J_{\mathrm{HH}}=16.2\right.$ $\mathrm{Hz}, 1 \mathrm{H}), 8.03\left(\mathrm{dd},{ }^{3} J_{\mathrm{HH}}=9.0 \mathrm{~Hz},{ }^{4} J_{\mathrm{HH}}=1.8 \mathrm{~Hz}, 2 \mathrm{H}\right), 7.91\left(\mathrm{dd},{ }^{3} J_{\mathrm{HH}}=7.8 \mathrm{~Hz}\right.$, $\left.{ }^{4} J_{\mathrm{HH}}=1.2 \mathrm{~Hz}, 1 \mathrm{H}\right), 7.75\left(\mathrm{~d},{ }^{3} J_{\mathrm{HH}}=7.8 \mathrm{~Hz}, 1 \mathrm{H}\right), 7.66\left(\mathrm{td},{ }^{3} J_{\mathrm{HH}}=7.2 \mathrm{~Hz},{ }^{4} J_{\mathrm{HH}}=1.2 \mathrm{~Hz} 1 \mathrm{H}\right), 7.60(\mathrm{~m}$, 2H), $7.52\left(\mathrm{t},{ }^{3} J_{\mathrm{HH}}=7.2 \mathrm{~Hz}, 2 \mathrm{H}\right), 7.37\left(\mathrm{~d},{ }^{3} J_{\mathrm{HH}}=15.6 \mathrm{~Hz}, 1 \mathrm{H}\right) .{ }^{13} \mathbf{C ~ N M R}\left(151 \mathrm{MHz}, \mathrm{CDCl}_{3}, 298 \mathrm{~K}\right): \delta=$ 191.8, 190.7, 141.4, 137.8, 137.4, 134.3, 134.0, 133.1, 132.3, 130.1, 128.9 (2C), 128.8(2C), 128.2, 127.5 . HRMS (ESI) $m / z$ calcd for $\mathrm{C}_{16} \mathrm{H}_{12} \mathrm{NaO}_{2}{ }^{+}(\mathrm{M}+\mathrm{Na})^{+}$, 259.0730; found: 259.0725.

(E)-2-(3-oxo-3-(o-tolyl)prop-1-en-1-yl)benzaldehyde (1b)<smiles>Cc1ccccc1C(=O)/C=C/c1ccccc1C=O</smiles>

$1 \mathrm{~b}$

Yield $0.177 \mathrm{~g}, 71 \%$; (petroleum ether/ethyl acetate = 2:1); light yellow solid; ${ }^{1} \mathbf{H}$ NMR $\left(600 \mathrm{MHz}, \mathrm{CDCl}_{3}, 298 \mathrm{~K}\right): \delta=10.24(\mathrm{~s}, 1 \mathrm{H}, \mathrm{CHO}), 8.29\left(\mathrm{~d},{ }^{3} J_{\mathrm{HH}}=16.2\right.$ $\mathrm{Hz}, 1 \mathrm{H}), 7.88\left(\mathrm{dd},{ }^{3} J_{\mathrm{HH}}=7.8 \mathrm{~Hz},{ }^{4} J_{\mathrm{HH}}=0.6 \mathrm{~Hz}, 1 \mathrm{H}\right), 7.72\left(\mathrm{~d},{ }^{3} J_{\mathrm{HH}}=7.8 \mathrm{~Hz}, 1 \mathrm{H}\right)$, $7.65\left(\mathrm{td},{ }^{3} J_{\mathrm{HH}}=7.2 \mathrm{~Hz},{ }^{4} J_{\mathrm{HH}}=0.6 \mathrm{~Hz}, 1 \mathrm{H}\right), 7.58\left(\mathrm{dd},{ }^{3} J_{\mathrm{HH}}=15.0 \mathrm{~Hz},{ }^{4} J_{\mathrm{HH}}=7.8\right.$ $\mathrm{Hz}, 1 \mathrm{H}), 7.41\left(\mathrm{td},{ }^{3} J_{\mathrm{HH}}=7.2 \mathrm{~Hz},{ }^{4} J_{\mathrm{HH}}=0.6 \mathrm{~Hz}, 1 \mathrm{H}\right), 7.31\left(\mathrm{t},{ }^{3} J_{\mathrm{HH}}=7.2 \mathrm{~Hz}, 2 \mathrm{H}\right), 7.03\left(\mathrm{~d},{ }^{3} J_{\mathrm{HH}}=16.2\right.$ $\mathrm{Hz}, 1 \mathrm{H}), 2.48$ (s, 3H, Me). ${ }^{13} \mathrm{C}$ NMR (151 MHz, $\left.\mathrm{CDCl}_{3}, 298 \mathrm{~K}\right): \delta=196.5,191.9,142.8,138.5,137.3$, 136.9, 134.1, 134.0, 132.8, 131.5, 131.4, 130.9, 130.2, 128.5, 128.1, 125.6, 20.5 (Me). HRMS (ESI) $m / z$ calcd for $\mathrm{C}_{17} \mathrm{H}_{15} \mathrm{O}_{2}{ }^{+}(\mathrm{M}+\mathrm{H})^{+}, 251.1067$; found: 251.1084 .

\section{(E)-2-(3-oxo-3-(m-tolyl)prop-1-en-1-yl)benzaldehyde (1c)}

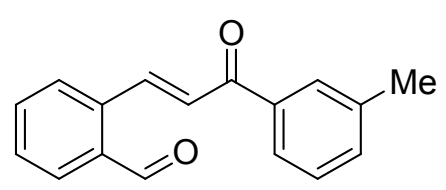

$1 \mathrm{c}$

Yield $0.187 \mathrm{~g}, 75 \%$; (petroleum ether/ethyl acetate $=4: 1$ ); light yellow solid; ${ }^{1} \mathbf{H}$ NMR $\left(600 \mathrm{MHz}, \mathrm{CDCl}_{3}, 298 \mathrm{~K}\right): \delta=10.35(\mathrm{~s}, 1 \mathrm{H}, \mathrm{CHO}), 8.54\left(\mathrm{~d},{ }^{3} J_{\mathrm{HH}}\right.$ $=15.6 \mathrm{~Hz}, 1 \mathrm{H}), 7.92\left(\mathrm{dd},{ }^{3} J_{\mathrm{HH}}=7.8, \mathrm{~Hz},{ }^{4} J_{\mathrm{HH}}=1.2 \mathrm{~Hz}, 1 \mathrm{H}\right), 7.84(\mathrm{~m}, 2 \mathrm{H})$, $7.75\left(\mathrm{~d},{ }^{3} J_{\mathrm{HH}}=7.8 \mathrm{~Hz}, 1 \mathrm{H}\right), 7.66\left(\mathrm{td},{ }^{3} J_{\mathrm{HH}}=7.2 \mathrm{~Hz},{ }^{4} J_{\mathrm{HH}}=1.2 \mathrm{~Hz}, 1 \mathrm{H}\right), 7.59$ $\left(\mathrm{t},{ }^{3} J_{\mathrm{HH}}=7.8 \mathrm{~Hz}, 1 \mathrm{H}\right), 7.40(\mathrm{~m}, 2 \mathrm{H}), 7.36\left(\mathrm{~d},{ }^{3} J_{\mathrm{HH}}=15.6 \mathrm{~Hz}, 1 \mathrm{H}\right), 2.45(\mathrm{~s}, 3 \mathrm{H}, \mathrm{Me}) .{ }^{13} \mathbf{C} \mathbf{~ N M R}(151$ $\left.\mathrm{MHz}, \mathrm{CDCl}_{3}, 298 \mathrm{~K}\right): \delta=191.8,190.9,141.2,138.6,137.8,137.5,134.4,134.0,133.9,132.2,130.1$, 
129.4, 128.6, 128.2, 127.7, 126.1, 21.5 (Me). HRMS (ESI) $m / z$ calcd for $\mathrm{C}_{17} \mathrm{H}_{15} \mathrm{O}_{2}{ }^{+}(\mathrm{M}+\mathrm{H})^{+}, 251.1067$; found: 251.1074 .

(E)-2-(3-oxo-3-(p-tolyl)prop-1-en-1-yl)benzaldehyde (1d)

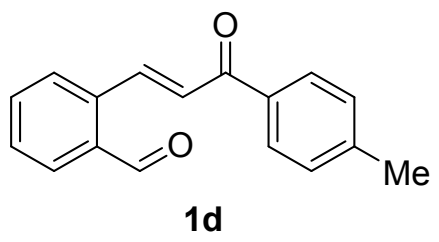

Yield $0.195 \mathrm{~g}, 78 \%$; (petroleum ether/ethyl acetate $=5: 1$ ); light yellow solid; ${ }^{1} \mathbf{H}$ NMR $\left(600 \mathrm{MHz}, \mathrm{CDCl}_{3}, 298 \mathrm{~K}\right): \delta=10.33(\mathrm{~s}, 1 \mathrm{H}, \mathrm{CHO}), 8.53\left(\mathrm{~d},{ }^{3} J_{\mathrm{HH}}\right.$

$=15.0 \mathrm{~Hz}, 1 \mathrm{H}), 7.94\left(\mathrm{~d},{ }^{3} J_{\mathrm{HH}}=7.8 \mathrm{~Hz}, 1 \mathrm{H}\right), 7.90\left(\mathrm{dd},{ }^{3} J_{\mathrm{HH}}=7.8 \mathrm{~Hz},{ }^{4} J_{\mathrm{HH}}=\right.$ $1.2 \mathrm{~Hz}, 1 \mathrm{H}), 7.74\left(\mathrm{~d},{ }^{3} J_{\mathrm{HH}}=7.8 \mathrm{~Hz}, 1 \mathrm{H}\right), 7.64\left(\mathrm{td},{ }^{3} J_{\mathrm{HH}}=7.8 \mathrm{~Hz},{ }^{4} J_{\mathrm{HH}}=1.2\right.$ $\mathrm{Hz}, 1 \mathrm{H}), 7.57\left(\mathrm{td},{ }^{3} J_{\mathrm{HH}}=7.8 \mathrm{~Hz},{ }^{4} J_{\mathrm{HH}}=1.2 \mathrm{~Hz} 1 \mathrm{H}\right), 7.37\left(\mathrm{~d},{ }^{3} J_{\mathrm{HH}}=15.6 \mathrm{~Hz}, 1 \mathrm{H}\right), 7.31\left(\mathrm{~d},{ }^{3} J_{\mathrm{HH}}=7.8\right.$ $\mathrm{Hz}, 2 \mathrm{H}), 2.43$ (s, 3H, Me). ${ }^{13} \mathrm{C}$ NMR (151 MHz, $\left.\mathrm{CDCl}_{3}, 298 \mathrm{~K}\right): \delta=191.8,190.0,144.0,140.7,137.5$, 135.2, 134.3, 134.0, 132.0, 130.0, 129.5 (2C), 129.0 (2C), 128.2, 127.5, 21.8 (Me). HRMS (ESI) $\mathrm{m} / z$ calcd for $\mathrm{C}_{17} \mathrm{H}_{15} \mathrm{O}_{2}{ }^{+}(\mathrm{M}+\mathrm{H})^{+}, 251.1067$; found: 251.1067 .

\section{(E)-2-(3-(4-methoxyphenyl)-3-oxoprop-1-en-1-yl)benzaldehyde (1e)}

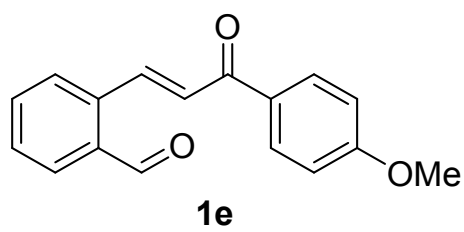

1e

Yield $0.239 \mathrm{~g}, 90 \%$; (petroleum ether/ethyl acetate = 5:1); light yellow solid; ${ }^{1} \mathbf{H}$ NMR $\left(600 \mathrm{MHz}, \mathrm{CDCl}_{3}, 298 \mathrm{~K}\right): \delta=10.35(\mathrm{~s}, 1 \mathrm{H}, \mathrm{CHO}), 8.52\left(\mathrm{~d},{ }^{3} J_{\mathrm{HH}}\right.$ $=15.6 \mathrm{~Hz}, 1 \mathrm{H}), 8.05(\mathrm{~m}, 2 \mathrm{H}), 7.91\left(\mathrm{dd},{ }^{3} J_{\mathrm{HH}}=7.8 \mathrm{~Hz},{ }^{4} J_{\mathrm{HH}}=1.2 \mathrm{~Hz}, 1 \mathrm{H}\right)$, $7.74\left(\mathrm{~d},{ }^{3} J_{\mathrm{HH}}=7.8 \mathrm{~Hz}, 1 \mathrm{H}\right), 7.65\left(\mathrm{td},{ }^{3} J_{\mathrm{HH}}=7.8 \mathrm{~Hz},{ }^{4} J_{\mathrm{HH}}=1.2 \mathrm{~Hz}, 1 \mathrm{H}\right)$, $7.57\left(\mathrm{td},{ }^{3} J_{\mathrm{HH}}=7.8 \mathrm{~Hz},{ }^{3} J_{\mathrm{HH}}=1.2 \mathrm{~Hz}, 1 \mathrm{H}\right), 7.37\left(\mathrm{~d},{ }^{3} J_{\mathrm{HH}}=15.6 \mathrm{~Hz}, 1 \mathrm{H}\right), 6.99(\mathrm{~m}, 2 \mathrm{H}), 3.89(\mathrm{~s}, 3 \mathrm{H}$, OMe). ${ }^{13} \mathrm{C}$ NMR (151 MHz, $\left.\mathrm{CDCl}_{3}, 298 \mathrm{~K}\right): \delta=191.8,188.7,163.7,140.3,137.7,134.3,134.0,131.9$, 131.2 (2C), 130.7, 129.9, 128.2, 127.5, 114.0 (2C), 55.6 (OMe). HRMS (ESI) $\mathrm{m} / z$ calcd for $\mathrm{C}_{17} \mathrm{H}_{15} \mathrm{O}_{3}{ }^{+}$ $(\mathrm{M}+\mathrm{H})^{+}, 267.1016$; found: 267.1021 .

(E)-2-(3-(2-chlorophenyl)-3-oxoprop-1-en-1-yl)benzaldehyde (1f)<smiles>O=Cc1ccccc1/C=C/C(=O)c1ccccc1Cl</smiles>

$1 f$

Yield 0.202 g, 75\%; (petroleum ether/ethyl acetate $=4: 1$ ); light yellow solid; ${ }^{1} \mathbf{H}$ NMR (600 MHz, $\left.\mathrm{CDCl}_{3}, 298 \mathrm{~K}\right): \delta=10.21(\mathrm{~s}, 1 \mathrm{H}, \mathrm{CHO}), 8.32\left(\mathrm{~d},{ }^{3} J_{\mathrm{HH}}=16.2\right.$ $\mathrm{Hz}, 1 \mathrm{H}), 7.86\left(\mathrm{dd},{ }^{3} J_{\mathrm{HH}}=7.8 \mathrm{~Hz},{ }^{4} J_{\mathrm{HH}}=1.2 \mathrm{~Hz}, 1 \mathrm{H}\right), 7.73\left(\mathrm{~d},{ }^{3} J_{\mathrm{HH}}=7.8 \mathrm{~Hz}, 1 \mathrm{H}\right)$, $7.64\left(\mathrm{td},{ }^{3} J_{\mathrm{HH}}=7.2 \mathrm{~Hz},{ }^{4} J_{\mathrm{HH}}=1.2 \mathrm{~Hz}, 1 \mathrm{H}\right), 7.59\left(\mathrm{td},{ }^{3} J_{\mathrm{HH}}=7.8,1.2 \mathrm{~Hz}, 1 \mathrm{H}\right), 7.54$ $\left(\mathrm{dd},{ }^{3} J_{\mathrm{HH}}=7.2 \mathrm{~Hz},{ }^{4} J_{\mathrm{HH}}=1.8 \mathrm{~Hz}, 1 \mathrm{H}\right), 7.45(\mathrm{~m}, 2 \mathrm{H}), 7.39\left(\mathrm{td},{ }^{3} J_{\mathrm{HH}}=7.2 \mathrm{~Hz},{ }^{4} J_{\mathrm{HH}}=1.8 \mathrm{~Hz}, 1 \mathrm{H}\right), 7.02$ $\left(\mathrm{d},{ }^{3} J_{\mathrm{HH}}=16.2 \mathrm{~Hz}, 1 \mathrm{H}\right) .{ }^{13} \mathbf{C}$ NMR $\left(151 \mathrm{MHz}, \mathrm{CDCl}_{3}, 298 \mathrm{~K}\right): \delta=193.9,192.0,143.2,138.6,136.5$, 134.1, 134.0, 132.9, 131.7, 131.4, 130.7 130.5, 134.3, 129.6, 128.1, 127.0. HRMS (ESI) $\mathrm{m} / z$ calcd for $\mathrm{C}_{16} \mathrm{H}_{12} \mathrm{ClO}_{2}^{+}(\mathrm{M}+\mathrm{H})^{+}, 271.0520$; found: 271.0524 . 
(E)-2-(3-(3-chlorophenyl)-3-oxoprop-1-en-1-yl)benzaldehyde (1g)<smiles>O=Cc1ccccc1/C=C/C(=O)c1cccc(Cl)c1</smiles>

$1 \mathrm{~g}$

Yield 0.189 g, 70\%; (petroleum ether/ethyl acetate $=5: 1$ ); light yellow solid;

${ }^{1} \mathbf{H}$ NMR $\left(600 \mathrm{MHz}, \mathrm{CDCl}_{3}, 298 \mathrm{~K}\right): \delta=10.30(\mathrm{~s}, 1 \mathrm{H}, \mathrm{CHO}), 8.58\left(\mathrm{~d},{ }^{3} J_{\mathrm{HH}}=\right.$ $16.2 \mathrm{~Hz}, 1 \mathrm{H}), 7.99\left(\mathrm{t},{ }^{3} J_{\mathrm{HH}}=1.8 \mathrm{~Hz}, 1 \mathrm{H}\right), 7.91\left(\mathrm{~d},{ }^{3} J_{\mathrm{HH}}=7.8 \mathrm{~Hz}, 2 \mathrm{H}\right), 7.75$ $\left(\mathrm{d},{ }^{3} J_{\mathrm{HH}}=7.2 \mathrm{~Hz}, 1 \mathrm{H}\right), 7.67\left(\mathrm{t},{ }^{3} J_{\mathrm{HH}}=7.2 \mathrm{~Hz}, 1 \mathrm{H}\right), 7.61\left(\mathrm{t},{ }^{3} J_{\mathrm{HH}}=7.8 \mathrm{~Hz}\right.$, $1 \mathrm{H}), 7.57\left(\mathrm{~d},{ }^{3} J_{\mathrm{HH}}=7.8 \mathrm{~Hz}, 1 \mathrm{H}\right), 7.46\left(\mathrm{t},{ }^{3} J_{\mathrm{HH}}=7.8 \mathrm{~Hz}, 1 \mathrm{H}\right), 7.29\left(\mathrm{~d},{ }^{3} J_{\mathrm{HH}}=16.2 \mathrm{~Hz}, 1 \mathrm{H}\right) .{ }^{13} \mathbf{C ~ N M R}$ $\left(151 \mathrm{MHz}, \mathrm{CDCl}_{3}, 298 \mathrm{~K}\right): \delta=192.0,189.5,142.5,139.3,136.9,135.1,134.4,134.0,133.0,132.8,130.3$, 130.1, 128.9, 128.2, 126.9, 126.8. HRMS (ESI) $\mathrm{m} / z$ calcd for $\mathrm{C}_{16} \mathrm{H}_{12} \mathrm{ClO}_{2}{ }^{+}(\mathrm{M}+\mathrm{H})^{+}, 271.0520$; found: 271.0514 .

(E)-2-(3-(4-chlorophenyl)-3-oxoprop-1-en-1-yl)benzaldehyde (1h)<smiles>O=Cc1ccccc1/C=C/C(=O)c1ccc(Cl)cc1</smiles>

$1 \mathrm{~h}$

Yield $0.216 \mathrm{~g}, 80 \%$; (petroleum ether/ethyl acetate $=5: 1$ ); light yellow solid; ${ }^{1} \mathbf{H}$ NMR $\left(600 \mathrm{MHz}, \mathrm{CDCl}_{3}, 298 \mathrm{~K}\right): \delta=10.25(\mathrm{~s}, 1 \mathrm{H}, \mathrm{CHO}), 8.49\left(\mathrm{~d},{ }^{3} \mathrm{JHH}_{\mathrm{HH}}=\right.$ $15.6 \mathrm{~Hz}, 1 \mathrm{H}), 7.92\left(\mathrm{~d},{ }^{3} J_{\mathrm{HH}}=8.4 \mathrm{~Hz}, 1 \mathrm{H}\right), 7.84\left(\mathrm{~d},{ }^{3} J_{\mathrm{HH}}=7.8 \mathrm{~Hz}, 1 \mathrm{H}\right), 7.67$ $\left(\mathrm{d},{ }^{3} J_{\mathrm{HH}}=7.8 \mathrm{~Hz}, 1 \mathrm{H}\right), 7.60\left(\mathrm{t},{ }^{3} J_{\mathrm{HH}}=7.8 \mathrm{~Hz}, 1 \mathrm{H}\right), 7.54\left(\mathrm{t},{ }^{3} J_{\mathrm{HH}}=7.2 \mathrm{~Hz}\right.$, $1 \mathrm{H}), 7.43\left(\mathrm{~d},{ }^{3} J_{\mathrm{HH}}=8.4 \mathrm{~Hz}, 2 \mathrm{H}\right), 7.23\left(\mathrm{~d},{ }^{3} \mathrm{JHH}_{\mathrm{HH}}=16.2 \mathrm{~Hz}, 1 \mathrm{H}\right) .{ }^{13} \mathbf{C ~ N M R}\left(151 \mathrm{MHz}, \mathrm{CDCl}_{3}, 298 \mathrm{~K}\right): \delta=$ 192.0, 189.6, 142.1, 139.6, 137.1, 136.1, 134.4, 134.0, 132.7, 130.3 (2C), 130.3, 129.1 (2C), 128.2, 127.0. HRMS (ESI) $m / z$ calcd for $\mathrm{C}_{16} \mathrm{H}_{12} \mathrm{ClO}_{2}{ }^{+}(\mathrm{M}+\mathrm{H})^{+}, 271.0520$; found: 271.0520 .

(E)-2-(3-(4-bromophenyl)-3-oxoprop-1-en-1-yl)benzaldehyde (1i)<smiles>O=Cc1ccccc1/C=C/C(=O)c1ccc(Br)cc1</smiles>

$1 \mathrm{i}$

Yield $0.282 \mathrm{~g}, 90 \%$; (petroleum ether/ethyl acetate $=5: 1$ ); lightyellow solid; ${ }^{1} \mathbf{H}$ NMR $\left(600 \mathrm{MHz}, \mathrm{CDCl}_{3}, 298 \mathrm{~K}\right): \delta=10.30(\mathrm{~s}, 1 \mathrm{H}, \mathrm{CHO}), 8.56\left(\mathrm{~d},{ }^{3} J_{\mathrm{HH}}\right.$ $=15.6 \mathrm{~Hz}, 1 \mathrm{H}), 7.90\left(\mathrm{~d},{ }^{3} J_{\mathrm{HH}}=9.1 \mathrm{~Hz}, 3 \mathrm{H}\right), 7.74\left(\mathrm{~d},{ }^{3} J_{\mathrm{HH}}=7.2 \mathrm{~Hz}, 1 \mathrm{H}\right), 7.66$ $(\mathrm{m}, 3 \mathrm{H}), 7.60\left(\mathrm{t},{ }^{3} J_{\mathrm{HH}}=6.6 \mathrm{~Hz}, 1 \mathrm{H}\right), 7.29\left(\mathrm{~d},{ }^{3} J_{\mathrm{HH}}=8.4 \mathrm{~Hz}, 2 \mathrm{H}\right), 7.23\left(\mathrm{~d},{ }^{3} J_{\mathrm{HH}}\right.$ $=15.6 \mathrm{~Hz}, 1 \mathrm{H}) .{ }^{13} \mathbf{C} \mathbf{N M R}\left(151 \mathrm{MHz}, \mathrm{CDCl}_{3}, 298 \mathrm{~K}\right): \delta=192.0,189.8,142.2,137.0,136.5,134.4,134.0$, 132.8, 132.1 (2C), 130.4 (2C), 130.3, 128.3, 128.2, 126.9. HRMS (ESI) $\mathrm{m} / z$ calcd for $\mathrm{C}_{16} \mathrm{H}_{12} \mathrm{BrO}_{2}{ }^{+}$ $(\mathrm{M}+\mathrm{H})^{+}, 315.0015$; found: 315.0015 .

\section{(E)-2-(3-(2-iodophenyl)-3-oxoprop-1-en-1-yl)benzaldehyde (1j)}<smiles>O=Cc1ccccc1/C=C/C(=O)c1ccccc1I</smiles>

$1 \mathbf{j}$

Yield 0.308 g, 85\%; (petroleum ether/ethyl acetate = 10:1); light yellow solid; ${ }^{1} \mathbf{H}$ NMR (600 MHz, $\left.\mathrm{CDCl}_{3}, 298 \mathrm{~K}\right): \delta=10.20(\mathrm{~s}, 1 \mathrm{H}, \mathrm{CHO}), 8.26\left(\mathrm{~d},{ }^{3} J_{\mathrm{HH}}=16.2\right.$ $\mathrm{Hz}, 1 \mathrm{H}), 7.39\left(\mathrm{~d},{ }^{3} J_{\mathrm{HH}}=8.4 \mathrm{~Hz}, 1 \mathrm{H}\right), 7.86\left(\mathrm{dd},{ }^{3} J_{\mathrm{HH}}=7.8 \mathrm{~Hz},{ }^{4} J_{\mathrm{HH}}=1.2 \mathrm{~Hz}, 1 \mathrm{H}\right)$, 
$7.73\left(\mathrm{~d},{ }^{3} J_{\mathrm{HH}}=7.8 \mathrm{~Hz}, 1 \mathrm{H}\right), 7.65\left(\mathrm{td},{ }^{3} J_{\mathrm{HH}}=7.8 \mathrm{~Hz},{ }^{4} J_{\mathrm{HH}}=1.2 \mathrm{~Hz}, 1 \mathrm{H}\right), 7.59\left(\mathrm{td},{ }^{3} J_{\mathrm{HH}}=7.2 \mathrm{~Hz},{ }^{4} J_{\mathrm{HH}}=\right.$ $1.2 \mathrm{~Hz}, 1 \mathrm{H}), 7.47(\mathrm{~m}, 2 \mathrm{H}), 7.18(\mathrm{~m}, 1 \mathrm{H}), 6.97\left(\mathrm{~d},{ }^{3} J_{\mathrm{HH}}=16.2 \mathrm{~Hz}, 1 \mathrm{H}\right) .{ }^{13} \mathbf{C} \mathbf{N M R}\left(151 \mathrm{MHz}, \mathrm{CDCl}_{3}, 298\right.$ $\mathrm{K}): \delta=196.2,192.0,144.2,144.0,140.1,136.5,134.1,134.0,133.1,131.7,130.5,130.1,128.9,128.2$, 128.1, 92.3. HRMS (ESI) $m / z$ calcd for $\mathrm{C}_{16} \mathrm{H}_{12} \mathrm{IO}_{2}{ }^{+}(\mathrm{M}+\mathrm{H})^{+}, 362.9876$; found: 362.9866 .

(E)-2-(3-(3-iodophenyl)-3-oxoprop-1-en-1-yl)benzaldehyde (1k)<smiles>O=Cc1ccccc1/C=C/C(=O)c1cccc(I)c1</smiles>

$1 \mathrm{k}$

Yield $0.253 \mathrm{~g}, 70 \%$; (petroleum ether/ethyl acetate $=10: 1$ ); light yellow solid; ${ }^{1} \mathbf{H}$ NMR $\left(600 \mathrm{MHz}, \mathrm{CDCl}_{3}, 298 \mathrm{~K}\right): \delta=10.32(\mathrm{~s}, 1 \mathrm{H}, \mathrm{CHO}), 8.58\left(\mathrm{~d},{ }^{3} J_{\mathrm{HH}}=\right.$ $15.6 \mathrm{~Hz}, 1 \mathrm{H}), 8.35\left(\mathrm{t},{ }^{3} J_{\mathrm{HH}}=1.8 \mathrm{~Hz}, 1 \mathrm{H}\right), 8.00(\mathrm{~m}, 1 \mathrm{H}), 7.93(\mathrm{~m}, 2 \mathrm{H}), 7.76(\mathrm{~d}$, $\left.{ }^{3} J_{\mathrm{HH}}=7.8 \mathrm{~Hz}, 1 \mathrm{H}\right), 7.68\left(\mathrm{td},{ }^{3} J_{\mathrm{HH}}=7.2 \mathrm{~Hz},{ }^{4} J_{\mathrm{HH}}=1.2 \mathrm{~Hz}, 1 \mathrm{H}\right), 7.62\left(\mathrm{td},{ }^{3} J_{\mathrm{HH}}\right.$ $\left.=7.8 \mathrm{~Hz},{ }^{4} J_{\mathrm{HH}}=1.2 \mathrm{~Hz}, 1 \mathrm{H}\right), 7.29(\mathrm{~m}, 2 \mathrm{H}) .{ }^{13} \mathbf{C ~ N M R}\left(151 \mathrm{MHz}, \mathrm{CDCl}_{3}, 298 \mathrm{~K}\right): \delta=191.9,189.3$, $142.5,141.8,139.6,137.7,136.9,134.4,134.0,132.8,130.5,130.4,128.2,127.9,126.7,94.6$. HRMS (ESI) $m / z$ calcd for $\mathrm{C}_{16} \mathrm{H}_{12} \mathrm{IO}_{2}{ }^{+}(\mathrm{M}+\mathrm{H})^{+}, 362.9876$; found: 362.9856 .

(E)-2-(3-(4-iodophenyl)-3-oxoprop-1-en-1-yl)benzaldehyde (11)<smiles>O=Cc1ccccc1/C=C/C(=O)c1ccc(I)cc1</smiles>

11

Yield $0.322 \mathrm{~g}, 89 \%$; (petroleum ether/ethyl acetate =10:1); light yellow solid; ${ }^{1} \mathbf{H}$ NMR $\left(600 \mathrm{MHz}, \mathrm{CDCl}_{3}, 298 \mathrm{~K}\right): \delta=10.25(\mathrm{~s}, 1 \mathrm{H}, \mathrm{CHO}), 8.49\left(\mathrm{~d},{ }^{3} J_{\mathrm{HH}}=\right.$ $15.6 \mathrm{~Hz}, 1 \mathrm{H}), 7.83(\mathrm{~m}, 3 \mathrm{H}), 7.68(\mathrm{~m}, 3 \mathrm{H}), 7.59\left(\mathrm{t},{ }^{3} J_{\mathrm{HH}}=7.2 \mathrm{~Hz}, 1 \mathrm{H}\right), 7.54(\mathrm{t}$, $\left.{ }^{3} J_{\mathrm{HH}}=7.8 \mathrm{~Hz}, 1 \mathrm{H}\right), 7.21\left(\mathrm{~d},{ }^{3} J_{\mathrm{HH}}=15.6 \mathrm{~Hz}, 1 \mathrm{H}\right), 7.29(\mathrm{~m}, 1 \mathrm{H}) .{ }^{13} \mathbf{C ~ N M R}(151$ $\left.\mathrm{MHz}, \mathrm{CDCl}_{3}, 298 \mathrm{~K}\right): \delta=192.0,190.2,142.3,138.1$ (2C), 137.1, 137.1, 134.4, 134.1, 132.8, 130.4 (2C), 130.3, 128.3, 126.9, 101.1. HRMS (ESI) $\mathrm{m} / z$ calcd for $\mathrm{C}_{16} \mathrm{H}_{12} \mathrm{IO}_{2}{ }^{+}(\mathrm{M}+\mathrm{H})^{+}, 362.9876$; found: 362.9896 . (E)-2-(3-oxo-3-(4-(trifluoromethyl)phenyl)prop-1-en-1-yl)benzaldehyde (1 m)

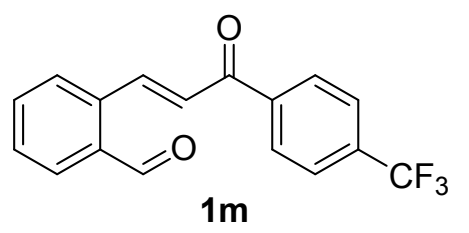

Yield $0.197 \mathrm{~g}, 65 \%$; (petroleum ether/ethyl acetate $=10: 1$ ); light yellow solid; ${ }^{1} \mathbf{H}$ NMR (600 MHz, $\left.\mathrm{CDCl}_{3}, 298 \mathrm{~K}\right): \delta=10.29$ (s, 1H, CHO), 8.59 $\left(\mathrm{d},{ }^{3} J_{\mathrm{HH}}=15.0 \mathrm{~Hz}, 1 \mathrm{H}\right), 8.13\left(\mathrm{~d},{ }^{3} J_{\mathrm{HH}}=8.4 \mathrm{~Hz}, 1 \mathrm{H}\right), 7.91\left(\mathrm{dd},{ }^{3} J_{\mathrm{HH}}=7.2\right.$ $\left.\mathrm{Hz},{ }^{4} J_{\mathrm{HH}}=1.2 \mathrm{~Hz}, 1 \mathrm{H}\right), 7.79\left(\mathrm{~d},{ }^{3} \mathrm{~J}_{\mathrm{HH}}=7.8 \mathrm{~Hz}, 1 \mathrm{H}\right), 7.75\left(\mathrm{~d},{ }^{3} \mathrm{~J}_{\mathrm{HH}}=7.8 \mathrm{~Hz}\right.$, $1 \mathrm{H}), 7.68\left(\mathrm{td},{ }^{3} J_{\mathrm{HH}}=7.2 \mathrm{~Hz},{ }^{4} J_{\mathrm{HH}}=1.2 \mathrm{~Hz}, 1 \mathrm{H}\right), 7.63\left(\mathrm{~d},{ }^{3} J_{\mathrm{HH}}=7.8 \mathrm{~Hz},{ }^{4} J_{\mathrm{HH}}=1.2 \mathrm{~Hz}, 1 \mathrm{H}\right), 7.29\left(\mathrm{~d},{ }^{3} J_{\mathrm{HH}}\right.$ $=15.6 \mathrm{~Hz}, 1 \mathrm{H}) .{ }^{13} \mathbf{C} \mathbf{N M R}\left(151 \mathrm{MHz}, \mathrm{CDCl}_{3}, 298 \mathrm{~K}\right): \delta=192.1,190.2,143.2,140.6,136.8,134.4,134.2$, 134.1, 133.2, 130.4, 129.2 (2C), 128.2, 126.9, 125.8 (q, $\left.{ }^{3} J_{\mathrm{CF}}=3.5 \mathrm{~Hz}, 1 \mathrm{C}\right), 124.6,122.8$. HRMS (ESI) $m / z$ calcd for $\mathrm{C}_{17} \mathrm{H}_{12} \mathrm{~F}_{3} \mathrm{O}_{2}{ }^{+}(\mathrm{M}+\mathrm{H})^{+}, 305.0784$; found: 305.0765 .

(E)-2-(3-(4-fluoro-3-methylphenyl)-3-oxoprop-1-en-1-yl)benzaldehyde (1n) 


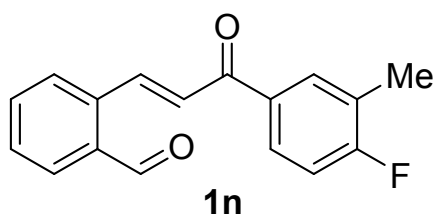

Yield $0.242 \mathrm{~g}, 90 \%$; (petroleum ether/ethyl acetate $=4: 1$ ); light yellow solid; ${ }^{1} \mathbf{H}$ NMR $\left(600 \mathrm{MHz}, \mathrm{CDCl}_{3}, 298 \mathrm{~K}\right): \delta=10.33(\mathrm{~s}, 1 \mathrm{H}, \mathrm{CHO}), 8.54\left(\mathrm{~d},{ }^{3} J_{\mathrm{HH}}\right.$ $=15.6 \mathrm{~Hz}, 1 \mathrm{H}), 7.92(\mathrm{~m}, 2 \mathrm{H}), 7.88(\mathrm{~m}, 1 \mathrm{H}), 7.74\left(\mathrm{~d},{ }^{3} J_{\mathrm{HH}}=7.8 \mathrm{~Hz}, 1 \mathrm{H}\right)$, $7.66\left(\mathrm{t},{ }^{3} J_{\mathrm{HH}}=8.4 \mathrm{~Hz}, 1 \mathrm{H}\right), 7.60\left(\mathrm{t},{ }^{3} J_{\mathrm{HH}}=7.8 \mathrm{~Hz}, 1 \mathrm{H}\right), 7.31\left(\mathrm{~d},{ }^{3} J_{\mathrm{HH}}=15.6 \mathrm{~Hz}, 1 \mathrm{H}\right), 7.13\left(\mathrm{t},{ }^{3} J_{\mathrm{HH}}=9.0\right.$ $\mathrm{Hz}, 1 \mathrm{H}), 2.37$ (m, 3H, Me). ${ }^{13} \mathbf{C}$ NMR (151 MHz, $\left.\mathrm{CDCl}_{3}, 298 \mathrm{~K}\right): \delta=191.9,189.5,165.4,163.7,141.6$, 137.4, 134.4, 134.1, 132.9, 132.5, 130.2, 128.9, 128.3, 127.4, 125.8, 115.6, 14.8 (Me). HRMS (ESI) $m / z$ calcd for $\mathrm{C}_{17} \mathrm{H}_{14} \mathrm{FO}_{2}{ }^{+}(\mathrm{M}+\mathrm{H})^{+}, 269.0972$; found: 269.0954 .

(E)-2-(3-oxo-3-(5,6,7,8-tetrahydronaphthalen-2-yl)prop-1-en-1-yl)benzaldehyde (10)

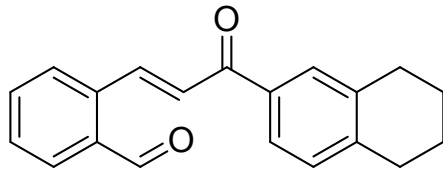

10 Yield $0.261 \mathrm{~g}, 90 \%$; (petroleum ether/ethyl acetate =4:1); light yellow solid; ${ }^{1} \mathbf{H}$ NMR $\left(600 \mathrm{MHz}, \mathrm{CDCl}_{3}, 298 \mathrm{~K}\right): \delta=10.38(\mathrm{~s}, 1 \mathrm{H}, \mathrm{CHO}), 8.55\left(\mathrm{~d},{ }^{3} J_{\mathrm{HH}}\right.$ $=15.6 \mathrm{~Hz}, 1 \mathrm{H}), 7.94\left(\mathrm{dd},{ }^{3} J_{\mathrm{HH}}=7.6 \mathrm{~Hz},{ }^{4} J_{\mathrm{HH}}=1.2 \mathrm{~Hz}, 1 \mathrm{H}\right), 7.78\left(\mathrm{~d},{ }^{3} J_{\mathrm{HH}}\right.$ $=8.4 \mathrm{~Hz}, 3 \mathrm{H}), 7.68\left(\mathrm{td},{ }^{3} J_{\mathrm{HH}}=7.2 \mathrm{~Hz},{ }^{4} J_{\mathrm{HH}}=1.2 \mathrm{~Hz}, 1 \mathrm{H}\right), 7.60\left(\mathrm{td},{ }^{3} J_{\mathrm{HH}}=\right.$

$\left.7.6 \mathrm{~Hz},{ }^{4} J_{\mathrm{HH}}=1.2 \mathrm{~Hz}, 1 \mathrm{H}\right), 7.38\left(\mathrm{~d},{ }^{3} J_{\mathrm{HH}}=15.6 \mathrm{~Hz}, 1 \mathrm{H}\right), 7.22\left(\mathrm{td},{ }^{3} J_{\mathrm{HH}}=8.4 \mathrm{~Hz},{ }^{4} J_{\mathrm{HH}}=1.2 \mathrm{~Hz}, 1 \mathrm{H}\right)$, $2.87\left(\mathrm{~m}, 4 \mathrm{H}, \mathrm{CH}_{2}\right), 1.86$ (m, 4H, $\left.\mathrm{CH}_{2}\right) .{ }^{13} \mathbf{C} \mathbf{N M R}\left(151 \mathrm{MHz}, \mathrm{CDCl}_{3}, 298 \mathrm{~K}\right): \delta=191.8,190.5,143.5$, $140.7,137.8,137.7,135.2$, 134.4, 134.1, 131.9, 129.9, 129.8, 129.6, 128.3, 127.9, 126.0, 29.8, 29.5, 23.1, 22.9. HRMS (ESI) $m / z$ calcd for $\mathrm{C}_{20} \mathrm{H}_{19} \mathrm{O}_{2}{ }^{+}(\mathrm{M}+\mathrm{H})^{+}, 291.1380$; found: 291.1360.

(E)-2-(3-(naphthalen-2-yl)-3-oxoprop-1-en-1-yl)benzaldehyde (1p)

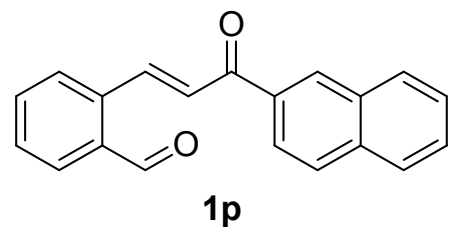

Yield $0.243 \mathrm{~g}, 85 \%$; (petroleum ether/ethyl acetate $=5: 1$ ); light yellow solid; ${ }^{1} \mathbf{H}$ NMR $\left(600 \mathrm{MHz}, \mathrm{CDCl}_{3}, 298 \mathrm{~K}\right): \delta=10.37(\mathrm{~s}, 1 \mathrm{H}, \mathrm{CHO}), 8.63\left(\mathrm{~d},{ }^{3} J_{\mathrm{HH}}\right.$ $=15.6 \mathrm{~Hz}, 1 \mathrm{H}), 8.60\left(\mathrm{~d},{ }^{3} J_{\mathrm{HH}}=0.6 \mathrm{~Hz}, 1 \mathrm{H}\right), 8.11\left(\mathrm{dd},{ }^{3} J_{\mathrm{HH}}=8.4 \mathrm{~Hz},{ }^{4} J_{\mathrm{HH}}\right.$ $=1.8 \mathrm{~Hz}, 1 \mathrm{H}), 8.03\left(\mathrm{dd},{ }^{3} J_{\mathrm{HH}}=7.8 \mathrm{~Hz},{ }^{4} J_{\mathrm{HH}}=0.6 \mathrm{~Hz}, 1 \mathrm{H}\right), 7.94(\mathrm{~m}, 3 \mathrm{H})$, $7.81\left(\mathrm{~d},{ }^{3} J_{\mathrm{HH}}=7.8 \mathrm{~Hz}, 1 \mathrm{H}\right), 7.69\left(\mathrm{td},{ }^{3} J_{\mathrm{HH}}=7.2 \mathrm{~Hz},{ }^{4} J_{\mathrm{HH}}=1.2 \mathrm{~Hz}, 1 \mathrm{H}\right), 7.63(\mathrm{~m}, 2 \mathrm{H}), 7.59(\mathrm{~m}, 1 \mathrm{H}), 7.51$ $\left(\mathrm{d},{ }^{3} J_{\mathrm{HH}}=15.6 \mathrm{~Hz}, 1 \mathrm{H}\right) .{ }^{13} \mathbf{C} \mathbf{N M R}\left(151 \mathrm{MHz}, \mathrm{CDCl}_{3}, 298 \mathrm{~K}\right): \delta=191.9,190.5,141.4,137.5,135.7$, 135.0, 134.4, 134.0, 132.6, 132.4, 130.6, 130.1, 129.7, 128.8, 128.6, 128.3, 127.9, 127.5, 127.0, 124.6. HRMS (ESI) $m / z$ calcd for $\mathrm{C}_{20} \mathrm{H}_{15} \mathrm{O}_{2}^{+}(\mathrm{M}+\mathrm{H})^{+}, 287.1067$; found: 287.1083 .

\section{(E)-2-(3-(3,4-dimethoxyphenyl)-3-oxoprop-1-en-1-yl)benzaldehyde (1q)}<smiles>COc1ccc(C(=O)/C=C/c2ccccc2C=O)cc1OC</smiles>

19
Yield 0.219 g, 74\%; (petroleum ether/ethyl acetate =4:1); light yellow solid; ${ }^{1} \mathbf{H}$ NMR $\left(600 \mathrm{MHz}, \mathrm{CDCl}_{3}, 298 \mathrm{~K}\right): \delta=10.27(\mathrm{~s}, 1 \mathrm{H}, \mathrm{CHO}), 8.48\left(\mathrm{~d},{ }^{3} J_{\mathrm{HH}}\right.$ $=15.0 \mathrm{~Hz}, 1 \mathrm{H}), 7.84(\mathrm{~m}, 1 \mathrm{H}), 7.69\left(\mathrm{~d},{ }^{3} J_{\mathrm{HH}}=7.8 \mathrm{~Hz}, 1 \mathrm{H}\right), 7.66\left(\mathrm{dd},{ }^{3} J_{\mathrm{HH}}\right.$ 
$\left.=8.4 \mathrm{~Hz},{ }^{4} J_{\mathrm{HH}}=1.8 \mathrm{~Hz}, 1 \mathrm{H}\right), 7.59(\mathrm{~m}, 2 \mathrm{H}), 7.52(\mathrm{~m}, 1 \mathrm{H}), 7.34\left(\mathrm{~d},{ }^{3} J_{\mathrm{HH}}=15.6 \mathrm{~Hz}, 1 \mathrm{H}\right), 6.89\left(\mathrm{~d},{ }^{3} J_{\mathrm{HH}}=\right.$ $8.4 \mathrm{~Hz}, 1 \mathrm{H}), 3.92(\mathrm{~m}, 6 \mathrm{H}, \mathrm{OMe}) .{ }^{13} \mathbf{C}$ NMR (151 MHz, $\left.\mathrm{CDCl}_{3}, 298 \mathrm{~K}\right): \delta=191.8,188.6,153.4,149.2$, $140.2,137.4,134.1,133.8,132.0,130.7,129.8,128.0,127.0,123.4,110.8,110.04,56.1,56.0$. HRMS (ESI) $m / z$ calcd for $\mathrm{C}_{18} \mathrm{H}_{17} \mathrm{O}_{4}{ }^{+}(\mathrm{M}+\mathrm{H})^{+}, 297.1121$; found: 297.1131 .

\section{(E)-2-(3-(3,4-dimethylphenyl)-3-oxoprop-1-en-1-yl)benzaldehyde (1r)}

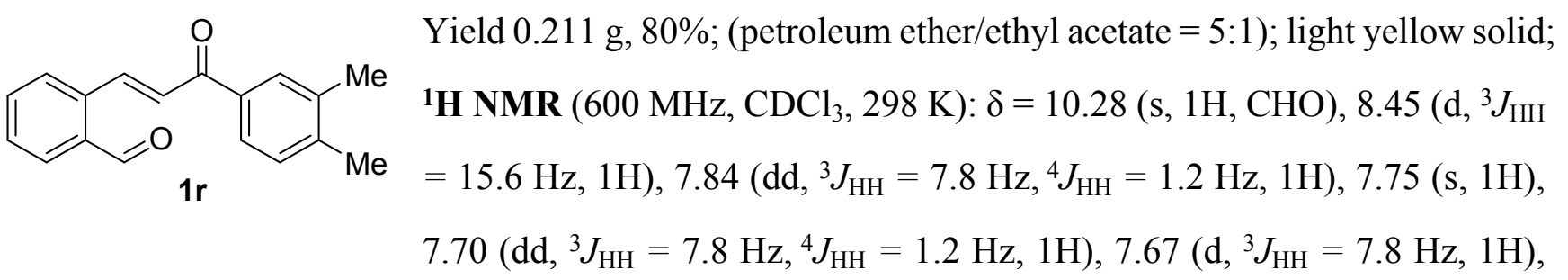

$7.58\left(\mathrm{td},{ }^{3} J_{\mathrm{HH}}=7.2 \mathrm{~Hz},{ }^{4} J_{\mathrm{HH}}=0.6 \mathrm{~Hz}, 1 \mathrm{H}\right), 7.50\left(\mathrm{t},{ }^{3} J_{\mathrm{HH}}=7.8 \mathrm{~Hz}, 1 \mathrm{H}\right), 7.29\left(\mathrm{~d},{ }^{3} J_{\mathrm{HH}}=15.6 \mathrm{~Hz}, 1 \mathrm{H}\right)$, $7.19\left(\mathrm{~d},{ }^{3} J_{\mathrm{HH}}=7.2 \mathrm{~Hz}, 1 \mathrm{H}\right), 2.28\left(\mathrm{~d},{ }^{3} J_{\mathrm{HH}}=6.0 \mathrm{~Hz}, 6 \mathrm{H}, \mathrm{Me}\right) .{ }^{13} \mathbf{C} \mathbf{N M R}\left(151 \mathrm{MHz}, \mathrm{CDCl}_{3}, 298 \mathrm{~K}\right): \delta=$ 191.8, 190.3, 142.8, 140.6, 137.7, 137.2, 135.6, 134.3, 134.0, 131.9, 130.1, 130.0, 129.9, 128.2, 127.7, 126.6, $20.2(\mathrm{Me}), 19.9$ (Me). HRMS (ESI) $\mathrm{m} / z$ calcd for $\mathrm{C}_{18} \mathrm{H}_{17} \mathrm{O}_{2}{ }^{+}(\mathrm{M}+\mathrm{H})^{+}, 265.1223$; found: 265.1203.

(E)-2-(3-(2,4-dimethylphenyl)-3-oxoprop-1-en-1-yl)benzaldehyde (1s)

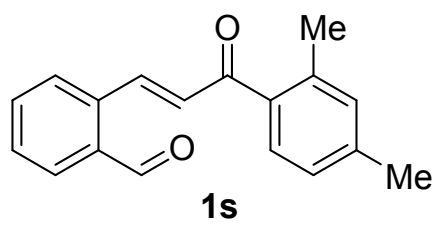

Yield $0.147 \mathrm{~g}, 60 \%$; (petroleum ether/ethyl acetate $=4: 1$ ); light yellow solid; ${ }^{1} \mathbf{H}$ NMR $\left(600 \mathrm{MHz}, \mathrm{CDCl}_{3}, 298 \mathrm{~K}\right): \delta=10.17(\mathrm{~s}, 1 \mathrm{H}, \mathrm{CHO}), 8.22\left(\mathrm{~d},{ }^{3} J_{\mathrm{HH}}\right.$ $=15.6 \mathrm{~Hz}, 1 \mathrm{H}), 7.78\left(\mathrm{~d},{ }^{3} J_{\mathrm{HH}}=7.8 \mathrm{~Hz}, 1 \mathrm{H}\right), 7.62\left(\mathrm{~d},{ }^{3} J_{\mathrm{HH}}=7.8 \mathrm{~Hz}, 1 \mathrm{H}\right)$, $7.55\left(\mathrm{t},{ }^{3} J_{\mathrm{HH}}=7.8 \mathrm{~Hz}, 1 \mathrm{H}\right), 7.48\left(\mathrm{t},{ }^{3} J_{\mathrm{HH}}=7.8 \mathrm{~Hz}, 1 \mathrm{H}\right), 7.44\left(\mathrm{~d},{ }^{3} J_{\mathrm{HH}}=8.4\right.$

$\mathrm{Hz}, 1 \mathrm{H}), 7.02\left(\mathrm{~d},{ }^{3} J_{\mathrm{HH}}=6.0 \mathrm{~Hz}, 2 \mathrm{H}\right), 6.97\left(\mathrm{~d},{ }^{3} J_{\mathrm{HH}}=15.6 \mathrm{~Hz}, 1 \mathrm{H}\right), 2.38(\mathrm{~s}, 3 \mathrm{H}, \mathrm{Me}), 2.28(\mathrm{~s}, 3 \mathrm{H}, \mathrm{Me})$.

${ }^{13} \mathrm{C}$ NMR (151 MHz, $\left.\mathrm{CDCl}_{3}, 298 \mathrm{~K}\right): \delta=195.6,191.9,141.8,141.5,138.0,137.2,135.5,134.1,134.0$, 132.5, 132.5, 131.4, 130.0, 129.1, 128.1, 126.2, 21.5 (Me), 20.6 (Me). HRMS (ESI) $m / z$ calcd for $\mathrm{C}_{18} \mathrm{H}_{17} \mathrm{O}_{2}^{+}(\mathrm{M}+\mathrm{H})^{+}, 265.1223$; found: 265.1223.

\section{(E)-2-(3-oxo-3-(thiophen-2-yl)prop-1-en-1-yl)benzaldehyde (1t)}

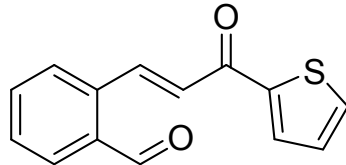

$1 \mathrm{t}$

Yield 0.169 g, 70\%; (petroleum ether/ethyl acetate $=2: 1$ ); light yellow solid; ${ }^{1} \mathbf{H}$ NMR $\left(600 \mathrm{MHz}, \mathrm{CDCl}_{3}, 298 \mathrm{~K}\right): \delta=10.35(\mathrm{~s}, 1 \mathrm{H}, \mathrm{CHO}), 8.61\left(\mathrm{~d},{ }^{3} J_{\mathrm{HH}}=15.6\right.$ $\mathrm{Hz}, 1 \mathrm{H}), 7.92(\mathrm{~m}, 2 \mathrm{H}), 7.74\left(\mathrm{~d},{ }^{3} J_{\mathrm{HH}}=7.8 \mathrm{~Hz}, 1 \mathrm{H}\right), 7.71\left(\mathrm{dd},{ }^{3} J_{\mathrm{HH}}=4.8 \mathrm{~Hz},{ }^{4} J_{\mathrm{HH}}\right.$ $=1.2 \mathrm{~Hz}, 1 \mathrm{H}), 7.66\left(\mathrm{td},{ }^{3} J_{\mathrm{HH}}=7.8 \mathrm{~Hz},{ }^{4} J_{\mathrm{HH}}=0.6 \mathrm{~Hz}, 1 \mathrm{H}\right), 7.59\left(\mathrm{t},{ }^{3} J_{\mathrm{HH}}=7.8 \mathrm{~Hz}\right.$,

$1 \mathrm{H}), 7.27\left(\mathrm{~d},{ }^{3} J_{\mathrm{HH}}=15.6 \mathrm{~Hz}, 1 \mathrm{H}\right), 7.20\left(\mathrm{dd},{ }^{3} J_{\mathrm{HH}}=4.8 \mathrm{~Hz},{ }^{4} J_{\mathrm{HH}}=4.2 \mathrm{~Hz}, 1 \mathrm{H}\right) .{ }^{13} \mathbf{C} \mathbf{N M R}(151 \mathrm{MHz}$, $\left.\mathrm{CDCl}_{3}, 298 \mathrm{~K}\right): \delta=191.8,182.1,145.0,140.5,137.2,134.4,134.4,134.0,132.5,132.2,130.1,128.4$, 
128.2, 127.1. HRMS (ESI) $m / z$ calcd for $\mathrm{C}_{14} \mathrm{H}_{11} \mathrm{O}_{2} \mathrm{~S}^{+}(\mathrm{M}+\mathrm{H})^{+}$, 243.0474; found: 243.0480.

(E)-5-fluoro-2-(3-oxo-3-phenylprop-1-en-1-yl)benzaldehyde (1u)<smiles>O=Cc1cc(F)ccc1/C=C/C(=O)c1ccccc1</smiles>

Yield $0.152 \mathrm{~g}, 60 \%$; (petroleum ether/ethyl acetate $=10: 1$ ); light yellow solid; ${ }^{1} \mathbf{H}$ NMR $\left(600 \mathrm{MHz}, \mathrm{CDCl}_{3}, 298 \mathrm{~K}\right): \delta=10.35(\mathrm{~s}, 1 \mathrm{H}, \mathrm{CHO}), 8.47\left(\mathrm{~d},{ }^{3} J_{\mathrm{HH}}=\right.$ $15.6 \mathrm{~Hz}, 1 \mathrm{H}), 8.02\left(\mathrm{~d},{ }^{3} J_{\mathrm{HH}}=7.2 \mathrm{~Hz}, 2 \mathrm{H}\right), 7.76\left(\mathrm{q},{ }^{3} J_{\mathrm{HH}}=4.8 \mathrm{~Hz}, 1 \mathrm{H}\right), 7.61$ $(\mathrm{m}, 2 \mathrm{H}), 7.52\left(\mathrm{t},{ }^{3} J_{\mathrm{HH}}=7.8 \mathrm{~Hz}, 2 \mathrm{H}\right), 7.38\left(\mathrm{t},{ }^{3} J_{\mathrm{HH}}=4.8 \mathrm{~Hz}, 1 \mathrm{H}\right), 7.36\left(\mathrm{~d},{ }^{3} J_{\mathrm{HH}}\right.$

$=15.6 \mathrm{~Hz}, 1 \mathrm{H}) .{ }^{13} \mathbf{C} \mathbf{N M R}\left(151 \mathrm{MHz}, \mathrm{CDCl}_{3}, 298 \mathrm{~K}\right): \delta=190.1,189.9,164.4,162.8,139.5,137.6,136.1$ $\left(\mathrm{d},{ }^{3} J_{\mathrm{CF}}=6.4 \mathrm{~Hz}, 1 \mathrm{C}\right), 133.3,130.4\left(\mathrm{~d},{ }^{3} J_{\mathrm{CF}}=8.0 \mathrm{~Hz}, 1 \mathrm{C}\right), 128.9(2 \mathrm{C}), 128.8(2 \mathrm{C}) 127.4\left(\mathrm{~d},{ }^{3} J_{\mathrm{CF}}=1.5\right.$ $\mathrm{Hz}, 1 \mathrm{C}), 121.4\left(\mathrm{~d},{ }^{3} J_{\mathrm{CF}}=21.9 \mathrm{~Hz}, 1 \mathrm{C}\right), 117.6,\left(\mathrm{~d},{ }^{3} J_{\mathrm{CF}}=22.7 \mathrm{~Hz}, 1 \mathrm{C}\right)$. HRMS (ESI) $\mathrm{m} / z$ calcd for $\mathrm{C}_{16} \mathrm{H}_{12} \mathrm{FO}_{2}^{+}(\mathrm{M}+\mathrm{H})^{+}, 255.0816$; found: 255.0806.

(E)-2-fluoro-6-(3-oxo-3-phenylprop-1-en-1-yl)benzaldehyde (1v)

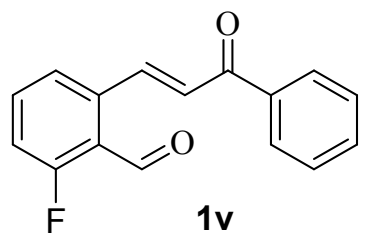

Yield 0.165 g, 65\%; (petroleum ether/ethyl acetate = 10:1); light yellow solid; ${ }^{1} \mathbf{H}$ NMR $\left(600 \mathrm{MHz}, \mathrm{CDCl}_{3}, 298 \mathrm{~K}\right): \delta=10.46(\mathrm{~s}, 1 \mathrm{H}, \mathrm{CHO}), 8.32\left(\mathrm{~d},{ }^{3} J_{\mathrm{HH}}=15.6\right.$ $\mathrm{Hz}, 1 \mathrm{H}), 7.94(\mathrm{~m}, 2 \mathrm{H}), 7.52(\mathrm{~m}, 2 \mathrm{H}), 7.42\left(\mathrm{q},{ }^{3} J_{\mathrm{HH}}=7.8 \mathrm{~Hz}, 3 \mathrm{H}\right), 7.18\left(\mathrm{~d},{ }^{3} J_{\mathrm{HH}}=\right.$ $15.6 \mathrm{~Hz}, 1 \mathrm{H}), 7.15(\mathrm{~m}, 1 \mathrm{H}) .{ }^{13} \mathbf{C}$ NMR $\left(151 \mathrm{MHz}, \mathrm{CDCl}_{3}, 298 \mathrm{~K}\right): \delta=191.1$, $188.3\left(\mathrm{~d},{ }^{3} J_{\mathrm{CF}}=10.4 \mathrm{~Hz}, 1 \mathrm{C}\right), 166.7,165.0,142.4\left(\mathrm{~d},{ }^{3} J_{\mathrm{CF}}=2.8 \mathrm{~Hz}, 1 \mathrm{C}\right), 138.7,137.6,135.5\left(\mathrm{~d},{ }^{3} J_{\mathrm{CF}}=\right.$ $10.2 \mathrm{~Hz}, 1 \mathrm{C}), 133.0(2 \mathrm{C}), 128.9\left(\mathrm{~d},{ }^{3} J_{\mathrm{CF}}=31.7 \mathrm{~Hz}, 1 \mathrm{C}\right), 127.7(2 \mathrm{C}), 124.1\left(\mathrm{~d},{ }^{3} J_{\mathrm{CF}}=3.2 \mathrm{~Hz}, 1 \mathrm{C}\right), 122.4$ $\left(\mathrm{d},{ }^{3} J_{\mathrm{CF}}=6.5 \mathrm{~Hz}, 1 \mathrm{C}\right), 117.5\left(\mathrm{~d},{ }^{3} J_{\mathrm{CF}}=21.7 \mathrm{~Hz}, 1 \mathrm{C}\right)$. HRMS (ESI) $m / z$ calcd for $\mathrm{C}_{16} \mathrm{H}_{12} \mathrm{FO}_{2}{ }^{+}(\mathrm{M}+\mathrm{H})^{+}$, 255.0816; found: 255.0816 .

\section{(E)-4-methyl-2-(3-oxo-3-phenylprop-1-en-1-yl)benzaldehyde (1w)}<smiles>Cc1ccc(C=O)c(/C=C/C(=O)c2ccccc2)c1</smiles>

$1 w$

Yield 0.202 g, 81\%; (petroleum ether/ethyl acetate = 5:1); light yellow solid;

${ }^{1} \mathbf{H}$ NMR $\left(600 \mathrm{MHz}, \mathrm{CDCl}_{3}, 298 \mathrm{~K}\right): \delta=10.28(\mathrm{~s}, 1 \mathrm{H}, \mathrm{CHO}), 8.55\left(\mathrm{~d},{ }^{3} J_{\mathrm{HH}}\right.$ $=15.6 \mathrm{~Hz}, 1 \mathrm{H}), 8.04(\mathrm{~m}, 2 \mathrm{H}), 7.81\left(\mathrm{~d},{ }^{3} J_{\mathrm{HH}}=7.8 \mathrm{~Hz}, 1 \mathrm{H}\right), 7.60\left(\mathrm{t},{ }^{3} J_{\mathrm{HH}}=\right.$ $7.2 \mathrm{~Hz}, 1 \mathrm{H}), 7.53(\mathrm{~m}, 3 \mathrm{H}), 7.39\left(\mathrm{~d},{ }^{3} J_{\mathrm{HH}}=7.8 \mathrm{~Hz}, 1 \mathrm{H}\right), 7.35\left(\mathrm{~d},{ }^{3} J_{\mathrm{HH}}=15.6\right.$ $\mathrm{Hz}, 1 \mathrm{H}), 2.48$ (s, 3H, Me). ${ }^{13} \mathbf{C}$ NMR (151 MHz, $\left.\mathrm{CDCl}_{3}, 298 \mathrm{~K}\right): \delta=191.5,190.8,145.1,141.7,137.8$, 137.4, 133.1, 132.6, 132.2, 130.9, 128.9, 128.8 (2C), 128.2 (2C), 127.2, 21.97 (Me). HRMS (ESI) $\mathrm{m} / \mathrm{z}$ calcd for $\mathrm{C}_{17} \mathrm{H}_{15} \mathrm{O}_{2}{ }^{+}(\mathrm{M}+\mathrm{H})^{+}, 251.1067$; found: 251.1084.

\section{(E)-5-methoxy-2-(3-oxo-3-phenylprop-1-en-1-yl)benzaldehyde (1x)}<smiles>[R]Cc1cc(OC)ccc1/C=C/C(=O)c1ccccc1</smiles>

Yield $0.212 \mathrm{~g}, 80 \%$; (petroleum ether/ethyl acetate $=5: 1$ ); light yellow solid; 
${ }^{1} \mathbf{H}$ NMR $\left(600 \mathrm{MHz}, \mathrm{CDCl}_{3}, 298 \mathrm{~K}\right): \delta=10.39(\mathrm{~s}, 1 \mathrm{H}, \mathrm{CHO}), 8.52\left(\mathrm{~d},{ }^{3} J_{\mathrm{HH}}=15.6 \mathrm{~Hz}, 1 \mathrm{H}\right), 8.02\left(\mathrm{~d},{ }^{3} J_{\mathrm{HH}}\right.$ $=7.2 \mathrm{~Hz}, 2 \mathrm{H}), 7.74\left(\mathrm{dd},{ }^{3} J_{\mathrm{HH}}=9.0 \mathrm{~Hz}, 1 \mathrm{H}\right), 7.59\left(\mathrm{t},{ }^{3} J_{\mathrm{HH}}=7.2 \mathrm{~Hz}, 1 \mathrm{H}\right), 7.50\left(\mathrm{t},{ }^{3} J_{\mathrm{HH}}=7.8 \mathrm{~Hz}, 2 \mathrm{H}\right), 7.41$ $\left(\mathrm{d},{ }^{3} J_{\mathrm{HH}}=3.0 \mathrm{~Hz}, 1 \mathrm{H}\right), 7.35\left(\mathrm{~d},{ }^{3} J_{\mathrm{HH}}=15.6 \mathrm{~Hz}, 1 \mathrm{H}\right), 7.18\left(\mathrm{dd},{ }^{3} J_{\mathrm{HH}}=8.4 \mathrm{~Hz},{ }^{4} J_{\mathrm{HH}}=3.0 \mathrm{~Hz}, 1 \mathrm{H}\right), 3.90$ (s, 3H, OMe). ${ }^{13} \mathbf{C}$ NMR (151 MHz, $\left.\mathrm{CDCl}_{3}, 298 \mathrm{~K}\right): \delta=191.0,190.4,161.3,140.1,138.0,135.7,133.0$, 130.0, 129.6, 128.8 (2C), 128.7 (2C), 125.4, 120.9, 114.5, 55.8 (OMe). HRMS (ESI) $\mathrm{m} / z$ calcd for $\mathrm{C}_{17} \mathrm{H}_{15} \mathrm{O}_{3}{ }^{+}(\mathrm{M}+\mathrm{H})^{+}, 267.1016$; found: 267.1025.

(E)-2-(3-oxo-3-phenylprop-1-en-1-yl)-5-(trifluoromethyl)benzaldehyde (1y)<smiles>O=Cc1cc(C(F)(F)F)ccc1/C=C/C(=O)c1ccccc1</smiles>

Yield $0.218 \mathrm{~g}, 72 \%$; (petroleum ether/ethyl acetate $=4: 1$ ); light yellow solid; ${ }^{1} \mathbf{H}$ NMR $\left(600 \mathrm{MHz}, \mathrm{CDCl}_{3}, 298 \mathrm{~K}\right): \delta=10.35(\mathrm{~s}, 1 \mathrm{H}, \mathrm{CHO}), 8.50\left(\mathrm{~d},{ }^{3} J_{\mathrm{HH}}\right.$ $=15.6 \mathrm{~Hz}, 1 \mathrm{H}), 8.15(\mathrm{~s}, 1 \mathrm{H}), 8.01\left(\mathrm{~d},{ }^{3} \mathrm{~J}_{\mathrm{HH}}=7.2 \mathrm{~Hz}, 2 \mathrm{H}\right), 7.86(\mathrm{~m}, 2 \mathrm{H}), 7.60$ $\left(\mathrm{t},{ }^{3} J_{\mathrm{HH}}=7.2 \mathrm{~Hz}, 1 \mathrm{H}\right), 7.50\left(\mathrm{t},{ }^{3} J_{\mathrm{HH}}=7.8 \mathrm{~Hz}, 1 \mathrm{H}\right), 7.44\left(\mathrm{~d},{ }^{3} J_{\mathrm{HH}}=15.6 \mathrm{~Hz}\right.$, 1H). ${ }^{13} \mathrm{C}$ NMR (151 MHz, $\left.\mathrm{CDCl}_{3}, 298 \mathrm{~K}\right): \delta=190.2,189.8,140.7,139.4$ (2C), 137.4, 134.4, 133.4 (2C), $132.08\left(\mathrm{q},{ }^{3} J_{\mathrm{CF}}=33.9 \mathrm{~Hz}, 1 \mathrm{C}\right), 130.32\left(\mathrm{q},{ }^{3} J_{\mathrm{CF}}=3.2 \mathrm{~Hz}, 1 \mathrm{C}\right), 129.05\left(\mathrm{~d},{ }^{3} J_{\mathrm{CF}}=18.6 \mathrm{~Hz}, 1 \mathrm{C}\right), 128.8(\mathrm{~d}$, $\left.{ }^{3} J_{\mathrm{CF}}=5.7 \mathrm{~Hz}, 1 \mathrm{C}\right), 128.6\left(\mathrm{q},{ }^{3} J_{\mathrm{CF}}=3.6 \mathrm{~Hz}, 1 \mathrm{C}\right), 126.1,124.2,122.4$. HRMS (ESI) $\mathrm{m} / \mathrm{z}$ calcd for $\mathrm{C}_{17} \mathrm{H}_{12} \mathrm{~F}_{3} \mathrm{O}_{2}^{+}(\mathrm{M}+\mathrm{H})^{+}, 305.0784$; found: 305.0786.

\section{(E)-1-(3-0xo-3-phenylprop-1-en-1-yl)-2-naphthaldehyde (1z)}

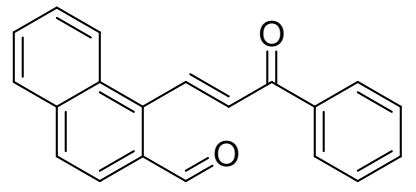

$1 z$

Yield $0.257 \mathrm{~g}, 90 \%$; (petroleum ether/ethyl acetate $=4: 1$ ); light yellow solid; ${ }^{1} \mathbf{H}$ NMR $\left(600 \mathrm{MHz}, \mathrm{CDCl}_{3}, 298 \mathrm{~K}\right): \delta=10.45(\mathrm{~s}, 1 \mathrm{H}, \mathrm{CHO}), 8.60\left(\mathrm{~d},{ }^{3} J_{\mathrm{HH}}=\right.$ $15.6 \mathrm{~Hz}, 1 \mathrm{H}), 8.21\left(\mathrm{~d},{ }^{3} J_{\mathrm{HH}}=8.4 \mathrm{~Hz}, 1 \mathrm{H}\right), 8.05(\mathrm{~m}, 3 \mathrm{H}), 7.95\left(\mathrm{t},{ }^{3} J_{\mathrm{HH}}=7.2 \mathrm{~Hz}\right.$, $1 \mathrm{H}), 7.69\left(\mathrm{td},{ }^{3} J_{\mathrm{HH}}=6.6 \mathrm{~Hz},{ }^{4} J_{\mathrm{HH}}=1.2 \mathrm{~Hz}, 1 \mathrm{H}\right), 7.63(\mathrm{~m}, 2 \mathrm{H}), 7.53\left(\mathrm{t},{ }^{3} J_{\mathrm{HH}}=\right.$ $7.8 \mathrm{~Hz}, 2 \mathrm{H}), 7.29\left(\mathrm{~d},{ }^{3} J_{\mathrm{HH}}=15.6 \mathrm{~Hz}, 1 \mathrm{H}\right) .{ }^{13} \mathbf{C} \mathbf{N M R}\left(151 \mathrm{MHz}, \mathrm{CDCl}_{3}, 298 \mathrm{~K}\right): \delta=191.7,188.6,140.0$, 138.7, 137.3, 135.8, 133.6, 133.5, 132.2, 131.6, 129.6, 129.3, 129.0 (2C), 128.9 (2C), 128.8, 127.7, 126.1, 123.3. HRMS (ESI) $m / z$ calcd for $\mathrm{C}_{20} \mathrm{H}_{14} \mathrm{NaO}_{2}{ }^{+}(\mathrm{M}+\mathrm{Na})^{+}, 309.0886$; found: 309.0874 .

\section{References}

[1] Maity, S.; Saha, M.; Hazra, G.; Ghorai, P. Switchable Chemoselectivity for Organocatalytic, Asymmetric Malononitrile Addition to ortho-Formyl Chalcones. Org. Lett. 2017, 19, 5872-5875.

[2] Lim, H. S.; Choi, Y. L.; Heo, J. N. Synthesis of Dibenzoxepine Lactams via a Cu-Catalyzed OnePot Etherification/Aldol Condensation Cascade Reaction: Application toward the Total Synthesis of 
Aristoyagonine. Org. Lett. 2013, 15, 4718-4721.

[3] Te Grotenhuis, C.; Das, B. G.; Kuijpers, P. F.; Hageman, W.; Trouwborst, M.; de Bruin, B. Catalytic 1,2-dihydronaphthalene and E-aryl-diene Synthesis via Co(III)-Carbene Radical and o-quinodimethane intermediates. Chem. Sci. 2017, 8, 8221-8230.

[4] Mondal, A.; Hazra, R.; Grover, J.; Raghu, M.; Ramasastry, S. S. V. Organophosphine-Catalyzed Intramolecular Hydroacylation of Activated Alkynes. ACS. Catal. 2018, 8, 2748-2753.

[5] Mishra, U. K.; Patel, K.; Ramasastry, S. S. V. Ring-Opening/Recyclization Cascades of Monoactivated Cyclopropanes. Org. Lett. 2020, 22, 3815-3819.

[6] Ravindra, B.; Das, B. G.; Ghorai, P. Organocatalytic, Enantioselective, Intramolecular oxa-Michael Reaction of Alkoxyboronate: A new Strategy for Enantioenriched 1-substituted 1,3dihydroisobenzofurans. Org. Lett. 2014, 16, 5580-5583.

[7] Youn, S. W.; Song, H. S.; Park, J. H. NHC-catalyzed Oxidative Cyclization Reaction for the Synthesis of 3-substituted Phthalides. Org. Biomol. Chem. 2014, 12, 2388-2393.

[8] Singha, S.; Kim, D.; Moon, H.; Wang, T.; Kim, K. H.; Shin, Y. H.; Jung, J.; Seo, E.; Lee, S. J.; Ahn, K. H. Toward a Selective, Sensitive, Fast-Responsive, and Biocompatible Two-Photon Probe for Hydrogen Sulfide in Live Cells. Anal. Chem. 2015, 87, 1188-1195.

[9] Jiang, B. L.; Ma, S. S.; Wang, M. L.; Liu, D. S.; Xu, B. H.; Zhang, S. J. Cobalt-Catalyzed Chemoselective Transfer Hydrogenation of $\mathrm{C}=\mathrm{C}$ and $\mathrm{C}=\mathrm{O}$ Bonds with Alkanols. ChemCatChem. 2019, $11,1701-1706$.

\section{Screening reaction conditions}

Table S1: Condition Optimization of the Reductive Enone-Tethered Aldehydes Cyclization. ${ }^{\mathrm{a}}$ 


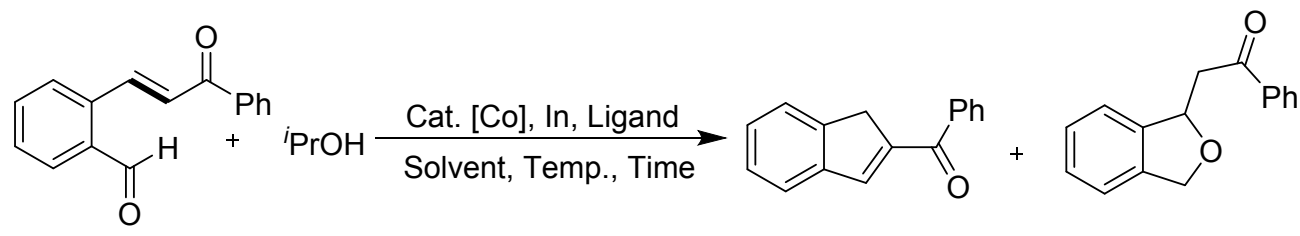

$1 \mathrm{a}$

$2 a$

3a

$\overbrace{\mathrm{R}}^{\mathrm{R}} \overbrace{\mathrm{R}}^{\mathrm{R}}$

$\operatorname{dcpp}(\mathrm{R}=\mathrm{Cy}, \mathrm{n}=1)$ dppp $(R=P h, n=1)$ dcpe $(R=C y, n=0)$<smiles>c1ccc(P(c2ccccc2)c2ccccc2P(c2ccccc2)c2ccccc2)cc1</smiles>

dppb<smiles>CC1(C)c2cccc(P(c3ccccc3)c3ccccc3)c2Oc2c(P(c3ccccc3)c3ccccc3)cccc21</smiles>

\begin{tabular}{|c|c|c|c|c|c|c|}
\hline Entry & {$[\mathrm{Co}]$} & $\begin{array}{l}\text { Ligand } \\
(\mathrm{mol} \%)\end{array}$ & $\begin{array}{l}i-\mathrm{PrOH} \\
\text { (equiv.) }\end{array}$ & Solvent & $\begin{array}{l}\text { Conv. } \\
(\%)^{b}\end{array}$ & 2a: $3 \mathbf{a}$ \\
\hline 1 & $\mathrm{CoI}_{2}$ & dcpp (15) & $(10)$ & toluene & 67 & $2: 1$ \\
\hline 2 & $\mathrm{CoI}_{2}$ & dcpp (15) & (15) & toluene & 72 & $2: 1$ \\
\hline 3 & $\mathrm{CoI}_{2}$ & dcpp (15) & (20) & toluene & 65 & $2: 1$ \\
\hline 4 & $\mathrm{CoI}_{2}$ & dcpp (20) & (15) & toluene & 69 & $2: 1$ \\
\hline $5^{c}$ & $\mathrm{CoI}_{2}$ & dcpp (15) & (15) & toluene & 60 & $2: 1$ \\
\hline $6^{d}$ & $\mathrm{CoI}_{2}$ & dcpp (15) & (15) & toluene & 43 & $2: 1$ \\
\hline 7 & $\mathrm{CoBr}_{2}$ & dcpp (15) & (15) & toluene & 46 & $3: 1$ \\
\hline 8 & $\mathrm{CoCl}_{2}$ & dcpp (15) & (15) & toluene & 55 & $2: 1$ \\
\hline 9 & $\mathrm{CoI}_{2}$ & dcpp (15) & (15) & dioxane & 43 & $3: 1$ \\
\hline 10 & $\mathrm{CoI}_{2}$ & dcpp (15) & (15) & $\mathrm{CH}_{3} \mathrm{CN}$ & 56 & $4: 1$ \\
\hline 11 & $\mathrm{CoI}_{2}$ & dcpp (15) & (15) & $\mathrm{THF}$ & 68 & $3: 1$ \\
\hline 12 & $\mathrm{CoI}_{2}$ & dcpp (15) & (15) & $\mathrm{DCE}$ & 43 & $2: 1$ \\
\hline 13 & $\mathrm{CoI}_{2}$ & dcpp (15) & (15) & DMSO & 48 & $2: 1$ \\
\hline 14 & $\mathrm{CoI}_{2}$ & dcpp (15) & (15) & DMF & 54 & $3: 1$ \\
\hline 15 & $\mathrm{CoI}_{2}$ & dppp (15) & (15) & toluene & 64 & $2: 1$ \\
\hline 16 & $\mathrm{CoI}_{2}$ & dppb (15) & (15) & toluene & 70 & $6: 1$ \\
\hline 17 & $\mathrm{CoI}_{2}$ & dcpe (15) & (15) & toluene & 37 & $1: 1$ \\
\hline 18 & $\mathrm{CoI}_{2}$ & xtanphos (15) & (15) & toluene & 31 & $3: 4$ \\
\hline 19 & $\mathrm{CoI}_{2}$ & dtpbx (15) & (15) & toluene & 91 & $4: 1$ \\
\hline $20^{e}$ & $\mathrm{CoI}_{2}$ & - & $(15)$ & toluene & 93 & $8: 1$ \\
\hline $21^{e}$ & $\mathrm{CoI}_{2}$ & dtpbx (15) & (15) & toluene & 0 & - \\
\hline $22^{g}$ & - & - & $(15)$ & toluene & 0 & - \\
\hline
\end{tabular}

${ }^{a}$ Reaction condition: $1 \mathrm{a}(0.1 \mathrm{mmol})$, [Co] $(10 \mathrm{~mol} \%)$, In $(40 \mathrm{~mol} \%)$, Solvent $(0.5 \mathrm{~mL}), 80^{\circ} \mathrm{C}$, 
$24 \mathrm{~h}$, under Ar. ${ }^{b}$ Conversions were determined by ${ }^{1} \mathrm{H}$ NMR using 2-methylindole as the internal standard. ${ }^{c} 100{ }^{\circ} \mathrm{C} .{ }^{d} 120{ }^{\circ} \mathrm{C} .{ }^{e} \mathrm{In}(0 \mathrm{~mol} \%) .{ }^{g} \mathrm{InI}_{3}(10 \mathrm{~mol} \%)$. THF $=$ Tetrahydrofuran; DCE $=$ 1,2-dichloroethane; DMSO = Dimethyl sulfoxide; $\mathrm{DMF}=N, N$-dimethylformamide.

General Procedure : Cobalt metal precursor [Co] $(0 \sim 10 \mathrm{mmol} \%)$ and Ligand (0 20 mmol \%) were added in a $25 \mathrm{~mL}$ pressure tube containing $0.5 \mathrm{~mL}$ degassed anhydrous toluene under argon. The mixture was stirred at room temperature for $15 \mathrm{~min}$ to form a dark blue solution. Then the indium powder (0 40 $\mathrm{mmol} \%$ ) was added and the mixture was stirred for $30 \mathrm{~min}$ to form an orange solution. To the resulting mixture were added enone-tethered aldehydes $(0.1 \mathrm{mmol})$ and $i$ - $\mathrm{PrOH}(0 \sim 20$ equiv $)$. The tube was closed and removed from the glove box and the mixture was stirred at $\mathrm{T}^{\circ} \mathrm{C}$ in a metal bath for $\mathrm{t} \mathrm{h}$. After completion of the reaction, 2-methylindole as an internal standard was added and filtered through a short pad of silica gel and concentrated. The residue was monitored by ${ }^{1} \mathrm{H}$ NMR for conversions and then purified by preparative thin layer chromatography (PTLC) with petroleum ether/ethyl acetate to afford the desired product.

\section{Cobalt-Catalyzed Cyclization Cascade of Enone-Tethered Aldehydes}

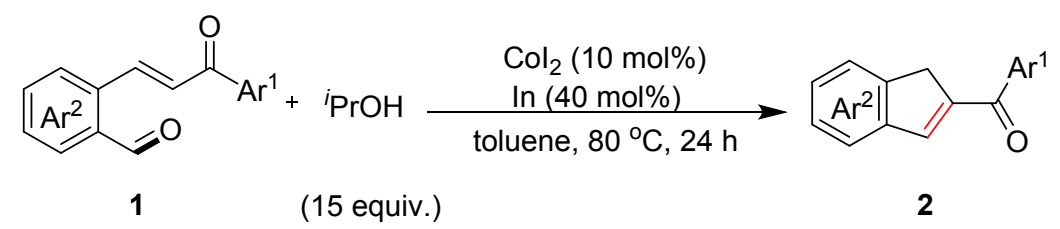

General Procedure : $\mathrm{CoI}_{2}(0.01 \mathrm{mmol})$ and enone-tethered aldehydes $(0.1 \mathrm{mmol})$ were added in a 25 $\mathrm{mL}$ pressure tube containing $0.5 \mathrm{~mL}$ degassed anhydrous toluene under argon. The mixture was stirred at room temperature for $15 \mathrm{~min}$ to form a dark blue solution. Then the indium powder $(0.04 \mathrm{mmol})$ was added and the mixture was stirred for $30 \mathrm{~min}$ to form an orange solution. To the resulting mixture was added $i$-PrOH (15 equiv.). The tube was closed and removed from the glove box and the mixture was stirred at $80{ }^{\circ} \mathrm{C}$ in a metal bath for $24 \mathrm{~h}$. After completion of the reaction, the solvent was evaporated to afford the crude product. The residue was purified by preparative thin layer chromatography (PTLC) with petroleum ether/ethyl acetate to afford the desired product.

\section{Analytical Data for Cyclization Products}




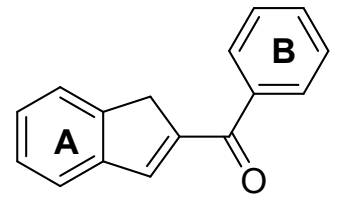

$2 \mathbf{a}$

Yield $17.9 \mathrm{mg}, 81 \%$; (petroleum ether/ethyl acetate = 5:1); Yellow solid; ${ }^{1} \mathbf{H}$ NMR $\left(600 \mathrm{MHz}, \mathrm{CDCl}_{3}, 298 \mathrm{~K}\right): \delta=7.85\left(\mathrm{dm}, 2 \mathrm{H},{ }^{3} J_{\mathrm{HH}}=8.5 \mathrm{~Hz}, o-\mathrm{Ph}^{\mathrm{B}}\right), 7.58(\mathrm{~m}, 1 \mathrm{H}$, $\left.\mathrm{Ph}^{\mathrm{A}}\right), 7.56\left(\mathrm{~m}, 1 \mathrm{H}, \mathrm{Ph}^{\mathrm{A}}\right), 7.54\left(\mathrm{~m}, 1 \mathrm{H}, \mathrm{Ph}^{\mathrm{A}}\right), 7.49(4)\left(\mathrm{m}, 2 \mathrm{H}, m-\mathrm{Ph}^{\mathrm{B}}\right), 7.49(1)(\mathrm{m}$, 1H, CH), $7.38\left(\mathrm{~m}, 1 \mathrm{H}, \mathrm{Ph}^{\mathrm{A}}\right), 7.35\left(\mathrm{~m}, 1 \mathrm{H}, p-\mathrm{Ph}^{\mathrm{B}}\right), 3.88\left(\mathrm{~m}, 2 \mathrm{H}, \mathrm{CH}_{2}\right) .{ }^{13} \mathbf{C}$ NMR $\left(151 \mathrm{MHz}, \mathrm{CDCl}_{3}, 298 \mathrm{~K}\right): \delta=192.8(\mathrm{C}=\mathrm{O}), 145.1\left(i-\mathrm{Ph}^{\mathrm{A}}\right), 145.0\left(i-\mathrm{Ph}^{\mathrm{A}}\right), 143.7\left(\mathrm{Ph}^{\mathrm{A}}\right), 143.0(\mathrm{CH}), 139.0$ (i-Cp), $131.9\left(i-\mathrm{Ph}^{\mathrm{B}}\right), 128.9\left(o-\mathrm{Ph}^{\mathrm{B}}\right), 128.4\left(m-\mathrm{Ph}^{\mathrm{B}}\right), 128.2\left(\mathrm{Ph}^{\mathrm{A}}\right), 127.0\left(p-\mathrm{Ph}^{\mathrm{B}}\right), 124.5\left(\mathrm{Ph}^{\mathrm{A}}\right), 124.0\left(\mathrm{Ph}^{\mathrm{A}}\right)$, $38.5\left(\mathrm{CH}_{2}\right)$. HRMS (ESI) $\mathrm{m} / z$ calcd for $\mathrm{C}_{16} \mathrm{H}_{12} \mathrm{NaO}^{+}(\mathrm{M}+\mathrm{Na})^{+}, 243.0780$; found: 243.0786

(1H-inden-2-yl)(o-tolyl)methanone (2b)

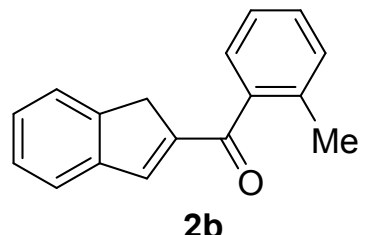

Yield $19.1 \mathrm{mg}, 82 \%$; (petroleum ether/ethyl acetate $=5: 1$ ); Yellow solid; ${ }^{1} \mathbf{H}$ $\operatorname{NMR}\left(600 \mathrm{MHz}, \mathrm{CDCl}_{3}, 298 \mathrm{~K}\right): \delta=7.50\left(\mathrm{~d},{ }^{3} J_{\mathrm{HH}}=7.8 \mathrm{~Hz}, 1 \mathrm{H}\right), 7.41\left(\mathrm{~d},{ }^{3} J_{\mathrm{HH}}\right.$ $=7.2 \mathrm{~Hz}, 1 \mathrm{H}), 7.31(\mathrm{~m}, 3 \mathrm{H}), 7.26\left(\mathrm{t},{ }^{3} J_{\mathrm{HH}}=7.2 \mathrm{~Hz}, 1 \mathrm{H}\right), 7.21(\mathrm{~m}, 2 \mathrm{H}), 7.18(\mathrm{~m}$, 1H), 3.79 (s, 2H, $\left.\mathrm{CH}_{2}\right), 2.30$ (s, 3H, Me). ${ }^{13} \mathrm{C} \mathrm{NMR}\left(151 \mathrm{MHz}, \mathrm{CDCl}_{3}, 298 \mathrm{~K}\right)$ : $\delta=195.6(\mathrm{C}=\mathrm{O}), 146.7,145.5,145.3,143.0,139.7,136.2,131.1,129.9,128.6,127.9,127.2,125.3,124.8$, 124.3, $37.8\left(\mathrm{CH}_{2}\right), 19.8(\mathrm{Me})$. HRMS (ESI) $\mathrm{m} / z$ calcd for $\mathrm{C}_{17} \mathrm{H}_{14} \mathrm{NaO}^{+}(\mathrm{M}+\mathrm{Na})^{+}, 257.0937$; found: 257.0929 .

(1H-inden-2-yl)(m-tolyl)methanone (2c)

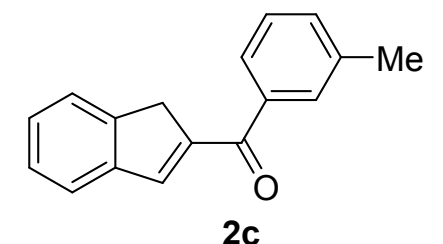

Yield $17.6 \mathrm{mg}, 75 \%$; (petroleum ether/ethyl acetate $=5: 1$ ); Yellow solid; ${ }^{\mathbf{1}} \mathbf{H}$ NMR $\left(600 \mathrm{MHz}, \mathrm{CDCl}_{3}, 298 \mathrm{~K}\right): \delta=7.63(\mathrm{~m}, 2 \mathrm{H}), 7.56\left(\mathrm{dd},{ }^{3} J_{\mathrm{HH}}=16.2 \mathrm{~Hz}\right.$, $\left.{ }^{4} J_{\mathrm{HH}}=7.2 \mathrm{~Hz}, 2 \mathrm{H}\right), 7.50(\mathrm{~s}, 1 \mathrm{H}), 7.38(\mathrm{~m}, 4 \mathrm{H}), 3.89\left(\mathrm{~s}, 2 \mathrm{H}, \mathrm{CH}_{2}\right), 2.45(\mathrm{~s}, 3 \mathrm{H}$, Me). ${ }^{13} \mathbf{C}$ NMR (151 MHz, $\left.\mathrm{CDCl}_{3}, 298 \mathrm{~K}\right): \delta=193.3(\mathrm{C}=\mathrm{O}), 145.4,145.2$, 143.7, 143.2, 139.2, 138.4, 132.8, 129.5, 128.3, 128.3, 127.2, 126.3, 124.7, 124.1, $38.7\left(\mathrm{CH}_{2}\right), 21.6(\mathrm{Me})$. HRMS (ESI) $m / z$ calcd for $\mathrm{C}_{17} \mathrm{H}_{14} \mathrm{NaO}^{+}(\mathrm{M}+\mathrm{Na})^{+}, 257.0937$; found: 257.0925.

(1H-inden-2-yl)(p-tolyl)methanone (2d)

Yield $19.5 \mathrm{mg}, 83 \%$; (petroleum ether/ethyl acetate $=5: 1$ ); Yellow solid; ${ }^{1} \mathbf{H}$ NMR $\left(600 \mathrm{MHz}, \mathrm{CDCl}_{3}, 298 \mathrm{~K}\right): \delta=7.77\left(\mathrm{~d},{ }^{3} J_{\mathrm{HH}}=7.8 \mathrm{~Hz}, 2 \mathrm{H}\right), 7.57\left(\mathrm{~d},{ }^{3} J_{\mathrm{HH}}\right.$ $=7.8 \mathrm{~Hz}, 1 \mathrm{H}), 7.54\left(\mathrm{~d},{ }^{3} J_{\mathrm{HH}}=7.2 \mathrm{~Hz}, 1 \mathrm{H}\right), 7.49(\mathrm{~s}, 1 \mathrm{H}), 7.37(\mathrm{~m}, 2 \mathrm{H}), 7.30(\mathrm{~d}$, $\left.{ }^{3} J_{\mathrm{HH}}=7.8 \mathrm{~Hz}, 2 \mathrm{H}\right), 3.89\left(\mathrm{~s}, 2 \mathrm{H}, \mathrm{CH}_{2}\right), 2.45$ (s, 3H, Me). ${ }^{13} \mathbf{C}$ NMR $(151 \mathrm{MHz}$, $\left.\mathrm{CDCl}_{3}, 298 \mathrm{~K}\right): \delta=192.8(\mathrm{C}=\mathrm{O}), 145.5,145.1,143.3,143.2,142.7,136.4,129.2$

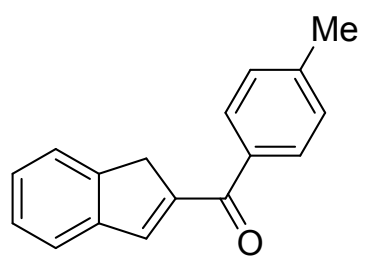

2d 
(2C), 129.1 (2C), 128.2, 127.1, 124.6, 123.9, $38.8\left(\mathrm{CH}_{2}\right), 21.7(\mathrm{Me})$. HRMS (ESI) $\mathrm{m} / \mathrm{z}$ calcd for $\mathrm{C}_{17} \mathrm{H}_{14} \mathrm{NaO}^{+}(\mathrm{M}+\mathrm{Na})^{+}, 257.0937$; found: 257.0938 .

(1H-inden-2-yl)(4-methoxyphenyl)methanone (2e)

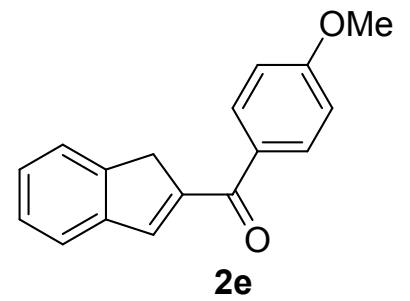

Yield $20.1 \mathrm{mg}, 80 \%$; (petroleum ether/ethyl acetate $=5: 1$ ); Yellow solid; ${ }^{1} \mathbf{H}$ NMR (600 MHz, $\left.\mathrm{CDCl}_{3}, 298 \mathrm{~K}\right): \delta=7.88\left(\mathrm{~d},{ }^{3} \mathrm{~J}_{\mathrm{HH}}=7.8 \mathrm{~Hz}, 2 \mathrm{H}\right), 7.55(\mathrm{dd}$, $\left.{ }^{3} J_{\mathrm{HH}}=15.6 \mathrm{~Hz},{ }^{4} J_{\mathrm{HH}}=7.2 \mathrm{~Hz}, 2 \mathrm{H}\right), 7.47(\mathrm{~s}, 1 \mathrm{H}), 7.37(\mathrm{~s}, 2 \mathrm{H}), 7.37(\mathrm{~m}, 2 \mathrm{H})$, $6.99\left(\mathrm{~d},{ }^{3} J_{\mathrm{HH}}=9.0 \mathrm{~Hz}, 2 \mathrm{H}\right), 3.90(\mathrm{~s}, 3 \mathrm{H}, \mathrm{OMe}), 3.88\left(\mathrm{t},{ }^{3} J_{\mathrm{HH}}=9.6 \mathrm{~Hz}, 2 \mathrm{H}\right.$,

$\left.\mathrm{CH}_{2}\right) .{ }^{13} \mathrm{C}$ NMR $\left(151 \mathrm{MHz}, \mathrm{CDCl}_{3}, 298 \mathrm{~K}\right): \delta=191.8(\mathrm{C}=\mathrm{O}), 163.0,145.6,145.0,143.3,142.5,131.7$, 131.4 (2C), 128.0, 127.1, 124.6, 123.9, 113.8 (2C), $55.6(\mathrm{OMe}), 38.9\left(\mathrm{CH}_{2}\right)$. HRMS (ESI) $\mathrm{m} / z$ calcd for $\mathrm{C}_{17} \mathrm{H}_{14} \mathrm{NaO}_{2}^{+}(\mathrm{M}+\mathrm{Na})^{+}, 273.0886$; found: 273.0894 .

\section{(2-chlorophenyl)(1H-inden-2-yl)methanone (2f)}

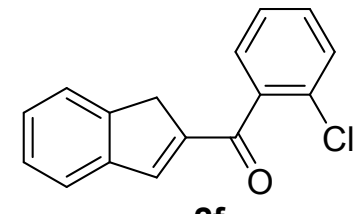

$2 f$

Yield $17.7 \mathrm{mg}, 70 \%$; (petroleum ether/ethyl acetate $=5: 1$ ); Yellow solid; ${ }^{1} \mathbf{H}$ NMR $\left(600 \mathrm{MHz}, \mathrm{CDCl}_{3}, 298 \mathrm{~K}\right): \delta=7.50\left(\mathrm{~d},{ }^{3} \mathrm{~J}_{\mathrm{HH}}=7.8 \mathrm{~Hz}, 1 \mathrm{H}\right), 7.41\left(\mathrm{dd},{ }^{3} J_{\mathrm{HH}}\right.$ $\left.=15.6 \mathrm{~Hz},{ }^{4} J_{\mathrm{HH}}=7.2 \mathrm{~Hz}, 2 \mathrm{H}\right), 7.34\left(\mathrm{td},{ }^{3} J_{\mathrm{HH}}=7.2 \mathrm{~Hz},{ }^{4} J_{\mathrm{HH}}=1.8 \mathrm{~Hz}, 2 \mathrm{H}\right), 7.31$ $(\mathrm{m}, 1 \mathrm{H}), 7.27\left(\mathrm{td},{ }^{3} J_{\mathrm{HH}}=7.8 \mathrm{~Hz},{ }^{4} J_{\mathrm{HH}}=1.8 \mathrm{~Hz}, 2 \mathrm{H}\right), 7.23(\mathrm{~s}, 1 \mathrm{H}), 3.79(\mathrm{~s}, 2 \mathrm{H}$, $\left.\mathrm{CH}_{2}\right) .{ }^{13} \mathrm{C}$ NMR $\left(151 \mathrm{MHz}, \mathrm{CDCl}_{3}, 298 \mathrm{~K}\right): \delta=190.9(\mathrm{C}=\mathrm{O}), 144.8,144.5,144.4,141.7,138.3,130.0$, 129.7, 129.1, 127.7, 127.6, 126.1, $125.5,123.7,123.3,36.3\left(\mathrm{CH}_{2}\right)$. HRMS (ESI) $\mathrm{m} / z$ calcd for $\mathrm{C}_{16} \mathrm{H}_{11} \mathrm{ClNaO}^{+}(\mathrm{M}+\mathrm{Na})^{+}, 277.0391$; found: 277.0356.

\section{(3-chlorophenyl)(1H-inden-2-yl)methanone (2g)}

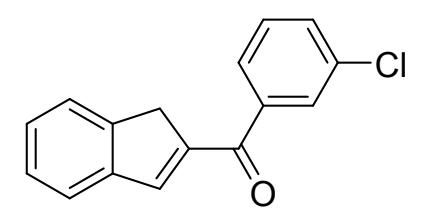

$2 \mathrm{~g}$

Yield $15.6 \mathrm{mg}, 61 \%$; (petroleum ether/ethyl acetate $=5: 1$ ); Yellow solid; ${ }^{1} \mathbf{H}$ NMR $\left(600 \mathrm{MHz}, \mathrm{CDCl}_{3}, 298 \mathrm{~K}\right): \delta=7.80\left(\mathrm{t},{ }^{3} J_{\mathrm{HH}}=1.8 \mathrm{~Hz}, 1 \mathrm{H}\right), 7.71(\mathrm{dt}$, $\left.{ }^{3} J_{\mathrm{HH}}=7.8 \mathrm{~Hz},{ }^{4} J_{\mathrm{HH}}=1.2 \mathrm{~Hz}, 1 \mathrm{H}\right), 7.56(\mathrm{~m}, 3 \mathrm{H}), 7.51(\mathrm{~s}, 1 \mathrm{H}), 7.44\left(\mathrm{t},{ }^{3} J_{\mathrm{HH}}=\right.$ $7.8 \mathrm{~Hz}, 1 \mathrm{H}), 7.40\left(\mathrm{td},{ }^{3} J_{\mathrm{HH}}=7.8 \mathrm{~Hz},{ }^{4} J_{\mathrm{HH}}=1.2 \mathrm{~Hz}, 1 \mathrm{H}\right), 7.37\left(\mathrm{t},{ }^{3} J_{\mathrm{HH}}=7.8\right.$

$\mathrm{Hz}, 1 \mathrm{H}), 3.88\left(\mathrm{~d},{ }^{3} J_{\mathrm{HH}}=1.2 \mathrm{~Hz}, 2 \mathrm{H}, \mathrm{CH}_{2}\right) .{ }^{13} \mathbf{C} \mathbf{N M R}\left(151 \mathrm{MHz}, \mathrm{CDCl}_{3}, 298 \mathrm{~K}\right): \delta=190.3(\mathrm{C}=\mathrm{O}), 144.1$, $143.6,143.3,141.8,139.6,133.6,130.8,128.7,127.9,127.5,126.1,125.9,123.6,123.2,37.5\left(\mathrm{CH}_{2}\right)$. HRMS (ESI) $m / z$ calcd for $\mathrm{C}_{16} \mathrm{H}_{11} \mathrm{ClNaO}^{+}(\mathrm{M}+\mathrm{Na})^{+}, 277.0391$; found: 277.0376 .

\section{(4-chlorophenyl)(1H-inden-2-yl)methanone (2h)}

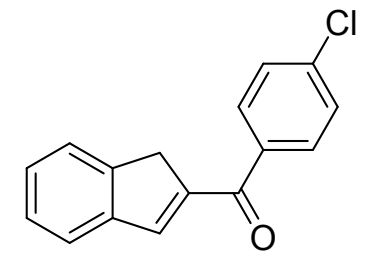

Yield $19.1 \mathrm{mg}, 75 \%$; (petroleum ether/ethyl acetate $=5: 1$ ); Yellow solid; ${ }^{1} \mathbf{H}$ NMR $\left(600 \mathrm{MHz}, \mathrm{CDCl}_{3}, 298 \mathrm{~K}\right): \delta=7.71\left(\mathrm{~d},{ }^{3} \mathrm{~J}_{\mathrm{HH}}=8.4 \mathrm{~Hz}, 2 \mathrm{H}\right), 7.49\left(\mathrm{dd},{ }^{3} \mathrm{~J}_{\mathrm{HH}}\right.$ 
$\left.=15.6 \mathrm{~Hz},{ }^{4} J_{\mathrm{HH}}=7.2 \mathrm{~Hz}, 2 \mathrm{H}\right), 7.40\left(\mathrm{~d},{ }^{3} J_{\mathrm{HH}}=8.4 \mathrm{~Hz}, 3 \mathrm{H}\right), 7.32\left(\mathrm{td},{ }^{3} J_{\mathrm{HH}}=7.2 \mathrm{~Hz},{ }^{4} J_{\mathrm{HH}}=1.2 \mathrm{~Hz}, 1 \mathrm{H}\right)$, $7.29\left(\mathrm{t},{ }^{3} J_{\mathrm{HH}}=7.2 \mathrm{~Hz}, 1 \mathrm{H}\right), 3.81\left(\mathrm{~s}, 2 \mathrm{H}, \mathrm{CH}_{2}\right) \cdot{ }^{13} \mathbf{C ~ N M R}\left(151 \mathrm{MHz}, \mathrm{CDCl}_{3}, 298 \mathrm{~K}\right): \delta=190.5(\mathrm{C}=\mathrm{O})$, 144.0, 143.8, 142.8, 141.8, 137.3, 136.2, 129.3 (2C), 127.7 (2C), 127.4, 126.1, 123.5, 123.0, $37.6\left(\mathrm{CH}_{2}\right)$.

HRMS (ESI) $m / z$ calcd for $\mathrm{C}_{16} \mathrm{H}_{11} \mathrm{ClNaO}^{+}(\mathrm{M}+\mathrm{Na})^{+}, 277.0391$; found: 277.0391 .

\section{(4-bromophenyl)(1H-inden-2-yl)methanone (2i)}

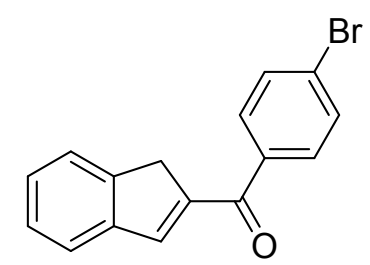

$2 \mathbf{i}$

Yield $24.2 \mathrm{mg}$, 81\%; (petroleum ether/ethyl acetate $=5: 1$ ); Yellow solid; ${ }^{1} \mathbf{H}$ $\operatorname{NMR}\left(600 \mathrm{MHz}, \mathrm{CDCl}_{3}, 298 \mathrm{~K}\right): \delta=7.71\left(\mathrm{dt},{ }^{3} \mathrm{~J}_{\mathrm{HH}}=8.4 \mathrm{~Hz},{ }^{4} J_{\mathrm{HH}}=1.8 \mathrm{~Hz}, 2 \mathrm{H}\right)$, $7.64\left(\mathrm{~d},{ }^{3} J_{\mathrm{HH}}=8.4 \mathrm{~Hz},{ }^{4} J_{\mathrm{HH}}=2.4 \mathrm{~Hz}, 2 \mathrm{H}\right), 7.57\left(\mathrm{dd},{ }^{3} J_{\mathrm{HH}}=16.2 \mathrm{~Hz},{ }^{4} J_{\mathrm{HH}}=7.2\right.$ $\mathrm{Hz}, 2 \mathrm{H}), 7.48(\mathrm{~s}, 1 \mathrm{H}), 7.40\left(\mathrm{td},{ }^{3} J_{\mathrm{HH}}=7.2 \mathrm{~Hz},{ }^{4} J_{\mathrm{HH}}=1.2 \mathrm{~Hz}, 1 \mathrm{H}\right), 7.36\left(\mathrm{t},{ }^{3} J_{\mathrm{HH}}\right.$ $=7.2 \mathrm{~Hz}, 1 \mathrm{H}), 3.88\left(\mathrm{~d},{ }^{3} J_{\mathrm{HH}}=1.2 \mathrm{~Hz}, 2 \mathrm{H}, \mathrm{CH}_{2}\right) \cdot{ }^{13} \mathbf{C ~ N M R}\left(151 \mathrm{MHz}, \mathrm{CDCl}_{3}, 298 \mathrm{~K}\right): \delta=190.7(\mathrm{C}=\mathrm{O})$, 144.0, 143.8, 142.9, 141.8, 136.6, 130.7 (2C), 129.4 (2C), 127.4, 126.1, 125.8, 123.5, 123.0, $37.5\left(\mathrm{CH}_{2}\right)$.

HRMS (ESI) $m / z$ calcd for $\mathrm{C}_{16} \mathrm{H}_{11} \mathrm{BrNaO}^{+}(\mathrm{M}+\mathrm{Na})^{+}, 320.9885$; found: 320.9858 .

(1H-inden-2-yl)(2-iodophenyl)methanone (2j)

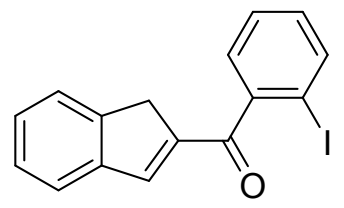

2j

Yield $24.2 \mathrm{mg}$, 70\%; (petroleum ether/ethyl acetate = 5:1); Yellow solid; ${ }^{1} \mathbf{H}$ NMR $\left(600 \mathrm{MHz}, \mathrm{CDCl}_{3}, 298 \mathrm{~K}\right): \delta=7.91\left(\mathrm{dd},{ }^{3} J_{\mathrm{HH}}=7.8 \mathrm{~Hz},{ }^{4} J_{\mathrm{HH}}=1.2 \mathrm{~Hz}, 1 \mathrm{H}\right), 7.57$ $\left(\mathrm{d},{ }^{3} J_{\mathrm{HH}}=7.8 \mathrm{~Hz}, 1 \mathrm{H}\right), 7.49\left(\mathrm{~d},{ }^{3} J_{\mathrm{HH}}=7.8 \mathrm{~Hz}, 1 \mathrm{H}\right), 7.44\left(\mathrm{td},{ }^{3} J_{\mathrm{HH}}=7.8 \mathrm{~Hz},{ }^{4} J_{\mathrm{HH}}\right.$ $=1.2 \mathrm{~Hz}, 1 \mathrm{H}), 7.39\left(\mathrm{td},{ }^{3} J_{\mathrm{HH}}=7.8 \mathrm{~Hz},{ }^{4} J_{\mathrm{HH}}=1.2 \mathrm{~Hz}, 1 \mathrm{H}\right), 7.35(\mathrm{~m}, 2 \mathrm{H}), 7.26(\mathrm{~s}$, $1 \mathrm{H}), 7.17\left(\mathrm{td},{ }^{3} J_{\mathrm{HH}}=7.8 \mathrm{~Hz},{ }^{4} J_{\mathrm{HH}}=1.8 \mathrm{~Hz}, 1 \mathrm{H}\right), 3.87\left(\mathrm{~s}, 2 \mathrm{H}, \mathrm{CH}_{2}\right) \cdot{ }^{13} \mathbf{C} \mathbf{~ N M R}\left(151 \mathrm{MHz}, \mathrm{CDCl}_{3}, 298\right.$ $\mathrm{K}): \delta=194.3(\mathrm{C}=\mathrm{O}), 146.3,145.7,145.2,144.9,142.8,139.8,131.0,128.9,128.0,127.9,127.3,124.8$, 124.5, 92.4, 37.6 $\left(\mathrm{CH}_{2}\right)$. HRMS (ESI) $m / z$ calcd for $\mathrm{C}_{16} \mathrm{H}_{11} \mathrm{INaO}^{+}(\mathrm{M}+\mathrm{Na})^{+}, 368.9747$; found: 368.9741 . (1H-inden-2-yl)(3-iodophenyl)methanone (2k)

Yield $23.9 \mathrm{mg}$, 69\%; (petroleum ether/ethyl acetate $=5: 1$ ); Yellow solid; ${ }^{1} \mathbf{H}$ NMR $\left(600 \mathrm{MHz}, \mathrm{CDCl}_{3}, 298 \mathrm{~K}\right): \delta=8.14\left(\mathrm{t},{ }^{3} J_{\mathrm{HH}}=1.8 \mathrm{~Hz}, 1 \mathrm{H}\right), 7.89\left(\mathrm{~d},{ }^{3} J_{\mathrm{HH}}\right.$ $=7.8 \mathrm{~Hz}, 1 \mathrm{H}), 7.77\left(\mathrm{~d},{ }^{3} J_{\mathrm{HH}}=7.8 \mathrm{~Hz}, 1 \mathrm{H}\right), 7.56(\mathrm{~m}, 2 \mathrm{H}), 7.49(\mathrm{~s}, 1 \mathrm{H}), 7.38(\mathrm{~m}$, $2 \mathrm{H}), 7.24\left(\mathrm{~d},{ }^{3} \mathrm{JHH}_{\mathrm{HH}}=7.8 \mathrm{~Hz}, 1 \mathrm{H}\right), 3.86\left(\mathrm{~s}, 2 \mathrm{H}, \mathrm{CH}_{2}\right) .{ }^{13} \mathbf{C ~ N M R}\left(151 \mathrm{MHz}, \mathrm{CDCl}_{3}\right.$,

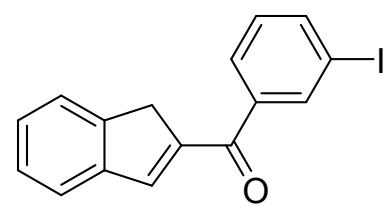

$2 \mathbf{k}$ $298 \mathrm{~K}): \delta=191.2(\mathrm{C}=\mathrm{O}), 145.2,144.7,144.4,142.9,140.9,140.8,137.7,130.2,128.6,128.1,127.3$, 124.7, 124.3, 94.3, $38.6\left(\mathrm{CH}_{2}\right)$. HRMS (ESI) $\mathrm{m} / z$ calcd for $\mathrm{C}_{16} \mathrm{H}_{11} \mathrm{INaO}^{+}(\mathrm{M}+\mathrm{Na})^{+}, 368.9747$; found: 368.9789 .

(1H-inden-2-yl)(4-iodophenyl)methanone (2l) 


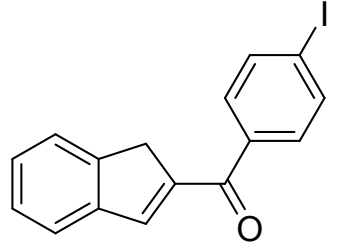

2I

Yield $24.6 \mathrm{mg}, 71 \%$; (petroleum ether/ethyl acetate $=5: 1$ ); Yellow solid; ${ }^{1} \mathbf{H}$ NMR $\left(600 \mathrm{MHz}, \mathrm{CDCl}_{3}, 298 \mathrm{~K}\right): \delta=7.78\left(\mathrm{~d},{ }^{3} \mathrm{~J}_{\mathrm{HH}}=8.4 \mathrm{~Hz}, 2 \mathrm{H}\right), 7.49(\mathrm{~m}, 4 \mathrm{H}), 7.41(\mathrm{~s}$, $1 \mathrm{H}), 7.32\left(\mathrm{td},{ }^{3} J_{\mathrm{HH}}=7.2 \mathrm{~Hz},{ }^{4} J_{\mathrm{HH}}=1.2 \mathrm{~Hz}, 1 \mathrm{H}\right), 7.29\left(\mathrm{t},{ }^{3} J_{\mathrm{HH}}=7.2 \mathrm{~Hz}, 1 \mathrm{H}\right), 3.80$ (s, $\left.2 \mathrm{H}, \mathrm{CH}_{2}\right) .{ }^{13} \mathbf{C}$ NMR $\left(151 \mathrm{MHz}, \mathrm{CDCl}_{3}, 298 \mathrm{~K}\right): \delta=192.0(\mathrm{C}=\mathrm{O}), 145.1,144.8$, 143.9, 142.8, 138.2, 137.7 (2C), 130.4 (2C), 128.4, 127.2, 124.6, 124.1, 99.2, $38.5\left(\mathrm{CH}_{2}\right)$. HRMS (ESI) $m / z$ calcd for $\mathrm{C}_{16} \mathrm{H}_{11} \mathrm{INaO}^{+}(\mathrm{M}+\mathrm{Na})^{+}, 368.9747$; found: 368.9756 .

\section{(1H-inden-2-yl)(4-(trifluoromethyl)phenyl)methanone (2m)}

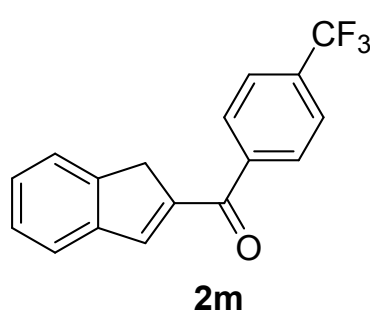

Yield $20.5 \mathrm{mg}, 71 \%$; (petroleum ether/ethyl acetate $=5: 1$ ); Yellow solid; ${ }^{1} \mathbf{H}$ NMR $\left(600 \mathrm{MHz}, \mathrm{CDCl}_{3}, 298 \mathrm{~K}\right): \delta=7.84\left(\mathrm{~d},{ }^{3} J_{\mathrm{HH}}=8.4 \mathrm{~Hz}, 2 \mathrm{H}\right), 7.69\left(\mathrm{~d},{ }^{3} J_{\mathrm{HH}}\right.$ $=8.4 \mathrm{~Hz}, 2 \mathrm{H}), 7.51\left(\mathrm{~d},{ }^{3} J_{\mathrm{HH}}=7.2 \mathrm{~Hz}, 1 \mathrm{H}\right), 7.48\left(\mathrm{~d},{ }^{3} J_{\mathrm{HH}}=7.2 \mathrm{~Hz}, 1 \mathrm{H}\right), 7.43$ $(\mathrm{s}, 1 \mathrm{H}), 7.34\left(\mathrm{td},{ }^{3} J_{\mathrm{HH}}=7.2,1.2 \mathrm{~Hz}, 1 \mathrm{H}\right), 7.29\left(\mathrm{~d},{ }^{3} J_{\mathrm{HH}}=7.2 \mathrm{~Hz}, 1 \mathrm{H}\right), 3.83(\mathrm{~d}$, $\left.{ }^{3} J_{\mathrm{HH}}=1.2 \mathrm{~Hz}, 2 \mathrm{H}, \mathrm{CH}_{2}\right) \cdot{ }^{13} \mathbf{C ~ N M R}\left(151 \mathrm{MHz}, \mathrm{CDCl}_{3}, 298 \mathrm{~K}\right): \delta=191.7(\mathrm{C}=\mathrm{O})$, $145.1,144.7\left(\mathrm{~d},{ }^{2} J_{\mathrm{CF}}=12.4 \mathrm{~Hz}\right), 142.7,142.1,133.5,133.2,129.1(2 \mathrm{C}), 128.7,127.2,125.5\left(\mathrm{q},{ }^{3} J_{\mathrm{CF}}=\right.$ $3.8 \mathrm{~Hz}, 2 \mathrm{C}), 124.6\left(\mathrm{q},{ }^{1} J_{\mathrm{CF}}=3.3 \mathrm{~Hz}, \mathrm{CF}_{3}\right), 124.2,122.9,38.4\left(\mathrm{CH}_{2}\right)$. HRMS (ESI) $\mathrm{m} / z$ calcd for $\mathrm{C}_{17} \mathrm{H}_{11} \mathrm{~F}_{3} \mathrm{NaO}^{+}(\mathrm{M}+\mathrm{Na})^{+}, 311.0654$; found: 311.0682 .

\section{(4-fluoro-3-methylphenyl)(1H-inden-2-yl)methanone (2n)}

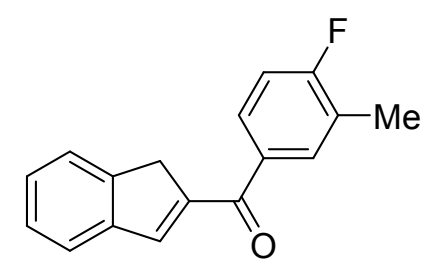

2n

Yield $18.1 \mathrm{mg}, 72 \%$; (petroleum ether/ethyl acetate = 5:1); Yellow solid; ${ }^{1} \mathbf{H}$ NMR $\left(600 \mathrm{MHz}, \mathrm{CDCl}_{3}, 298 \mathrm{~K}\right): \delta=7.72\left(\mathrm{dd},{ }^{3} J_{\mathrm{HH}}=7.8 \mathrm{~Hz},{ }^{4} J_{\mathrm{HH}}=1.2 \mathrm{~Hz}\right.$, 1H), $7.68(\mathrm{~m}, 1 \mathrm{H}), 7.56\left(\mathrm{t},{ }^{3} J_{\mathrm{HH}}=8.4 \mathrm{~Hz}, 2 \mathrm{H}\right), 7.47(\mathrm{~s}, 1 \mathrm{H}), 7.37(\mathrm{~m}, 2 \mathrm{H})$, $7.10\left(\mathrm{t},{ }^{3} \mathrm{HH}_{\mathrm{HH}}=9.0 \mathrm{~Hz}, 1 \mathrm{H}\right), 3.88\left(\mathrm{~s}, 2 \mathrm{H}, \mathrm{CH}_{2}\right), 2.36$ (s, 3H, Me). ${ }^{13} \mathbf{C} \mathbf{~ N M R}$ $\left(151 \mathrm{MHz}, \mathrm{CDCl}_{3}, 298 \mathrm{~K}\right): \delta=191.8(\mathrm{C}=\mathrm{O}), 164.7,163.0,145.2,143.4$, 143.0, 135.0, 132.7, 128.9, 128.3, 127.2, 125.4, 124.6, 124.0, 115.1, $38.8\left(\mathrm{CH}_{2}\right), 14.7(\mathrm{Me})$. HRMS (ESI) $m / z$ calcd for $\mathrm{C}_{17} \mathrm{H}_{13} \mathrm{FNaO}^{+}(\mathrm{M}+\mathrm{Na})^{+}, 275.0843$; found: 275.0872 .

(1H-inden-2-yl)(5,6,7,8-tetrahydronaphthalen-2-yl)methanone (2o)

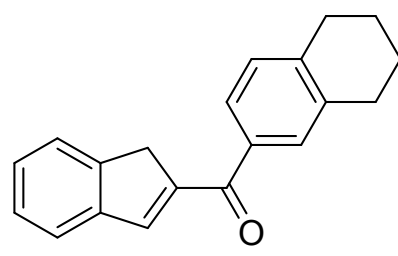

20

Yield $21.6 \mathrm{mg}$, 79\%; (petroleum ether/ethyl acetate $=5: 1$ ); Yellow solid; ${ }^{1} \mathbf{H}$ NMR $\left(600 \mathrm{MHz}, \mathrm{CDCl}_{3}, 298 \mathrm{~K}\right): \delta=7.49\left(\mathrm{~d},{ }^{3} J_{\mathrm{HH}}=6.6 \mathrm{~Hz}, 3 \mathrm{H}\right), 7.46(\mathrm{dd}$, $\left.{ }^{3} J_{\mathrm{HH}}=6.6,1.2 \mathrm{~Hz}, 1 \mathrm{H}\right), 7.41(\mathrm{~s}, 1 \mathrm{H}), 7.29(\mathrm{~m}, 2 \mathrm{H}), 7.09\left(\mathrm{~d},{ }^{3} J_{\mathrm{HH}}=8.4 \mathrm{~Hz}\right.$, 1H), $3.80\left(\mathrm{~s}, 2 \mathrm{H}, \mathrm{CH}_{2}\right), 2.77$ (s, 4H), $1.77(\mathrm{~m}, 4 \mathrm{H}) .{ }^{13} \mathbf{C ~ N M R}\left(151 \mathrm{MHz}, \mathrm{CDCl}_{3}\right.$, $298 \mathrm{~K}): \delta=193.0(\mathrm{C}=\mathrm{O}), 145.5,145.0,143.2,143.0,141.9,137.4,136.3,129.8,129.0,128.0,127.0$, 
126.2, 124.5, 123.8, $38.7\left(\mathrm{CH}_{2}\right), 29.6,29.5,23.1,22.9$. HRMS (ESI) $m / z$ calcd for $\mathrm{C}_{20} \mathrm{H}_{18} \mathrm{NaO}^{+}(\mathrm{M}+\mathrm{Na})^{+}$, 297.1250; found: 297.1254 .

\section{(1H-inden-2-yl)(naphthalen-2-yl)methanone (2p)}

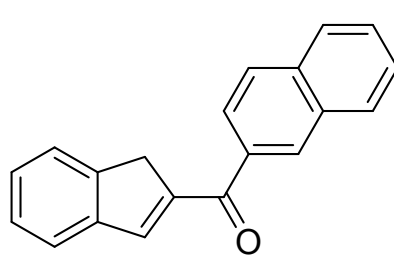

$2 p$

Yield $19.5 \mathrm{mg}, 72 \%$; (petroleum ether/ethyl acetate = 5:1); Yellow solid; ${ }^{1} \mathbf{H}$ NMR $\left(600 \mathrm{MHz}, \mathrm{CDCl}_{3}, 298 \mathrm{~K}\right): \delta=8.37(\mathrm{~s}, 1 \mathrm{H}), 7.95(\mathrm{~m}, 4 \mathrm{H}), 7.60(\mathrm{~m}, 5 \mathrm{H})$, $7.41\left(\mathrm{td},{ }^{3} J_{\mathrm{HH}}=7.8,1.2 \mathrm{~Hz}, 1 \mathrm{H}\right), 7.38\left(\mathrm{dd},{ }^{3} J_{\mathrm{HH}}=7.8,0.6 \mathrm{~Hz}, 1 \mathrm{H}\right), 3.96(\mathrm{~s}$, $\left.2 \mathrm{H}, \mathrm{CH}_{2}\right) .{ }^{13} \mathbf{C} \mathbf{N M R}\left(151 \mathrm{MHz}, \mathrm{CDCl}_{3}, 298 \mathrm{~K}\right): \delta=193.0(\mathrm{C}=\mathrm{O}), 145.5,145.2$, $143.8,143.2,136.3,135.2,132.5,130.1,129.4,128.5,128.3,128.1,127.9$, 127.2, 126.9, 125.4, 124.7, 124.1, $38.80\left(\mathrm{CH}_{2}\right)$. HRMS (ESI) $\mathrm{m} / z$ calcd for $\mathrm{C}_{20} \mathrm{H}_{14} \mathrm{NaO}^{+}(\mathrm{M}+\mathrm{Na})^{+}$, 293.0937; found: 293.0929 .

\section{(3,4-dimethoxyphenyl)(1H-inden-2-yl)methanone (2q)}

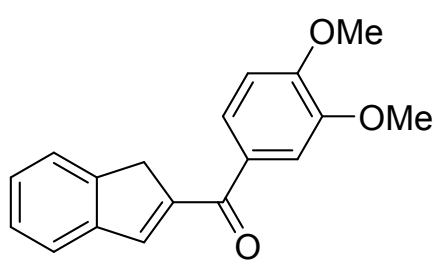

$2 q$

Yield $20.7 \mathrm{mg}, 74 \%$; (petroleum ether/ethyl acetate = 5:1); Yellow solid; ${ }^{1} \mathbf{H}$ NMR $\left(600 \mathrm{MHz}, \mathrm{CDCl}_{3}, 298 \mathrm{~K}\right): \delta=7.50\left(\mathrm{~d},{ }^{3} J_{\mathrm{HH}}=6.6 \mathrm{~Hz}, 1 \mathrm{H}\right), 7.47(\mathrm{dt}$, $\left.{ }^{3} J_{\mathrm{HH}}=7.2 \mathrm{~Hz},{ }^{4} J_{\mathrm{HH}}=1.8 \mathrm{~Hz}, 2 \mathrm{H}\right), 7.42(\mathrm{~s}, 1 \mathrm{H}), 7.38\left(\mathrm{~d},{ }^{3} J_{\mathrm{HH}}=1.8 \mathrm{~Hz}, 1 \mathrm{H}\right)$, $7.30(\mathrm{~m}, 2 \mathrm{H}), 6.86\left(\mathrm{~d},{ }^{3} J_{\mathrm{HH}}=8.4 \mathrm{~Hz}, 1 \mathrm{H}\right), 3.89\left(\mathrm{~d},{ }^{3} J_{\mathrm{HH}}=9.0 \mathrm{~Hz}, 6 \mathrm{H}, \mathrm{OMe}\right)$, $3.82(\mathrm{~s}, 2 \mathrm{H}) .{ }^{13} \mathrm{C}$ NMR $\left(151 \mathrm{MHz}, \mathrm{CDCl}_{3}, 298 \mathrm{~K}\right): \delta=190.6(\mathrm{C}=\mathrm{O}), 151.7$, 148.1 , 144.3, 143.8, 142.1, 141.3, 130.6, 126.9, 125.9, 123.5, 122.7, 122.6, 110.5, 108.9, 55.1 (OMe), 55.0 (OMe), $37.9\left(\mathrm{CH}_{2}\right)$. HRMS (ESI) $\mathrm{m} / z$ calcd for $\mathrm{C}_{18} \mathrm{H}_{16} \mathrm{NaO}_{3}{ }^{+}(\mathrm{M}+\mathrm{Na})^{+}, 303.0992$; found: 303.0990 . (3,4-dimethylphenyl)(1H-inden-2-yl)methanone (2r)

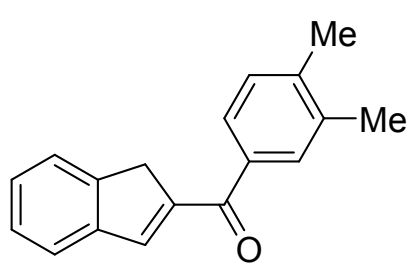

$2 r$

Yield $20.4 \mathrm{mg}, 82 \%$; (petroleum ether/ethyl acetate = 5:1); Yellow solid; ${ }^{1} \mathbf{H}$ NMR $\left(600 \mathrm{MHz}, \mathrm{CDCl}_{3}, 298 \mathrm{~K}\right): \delta=7.63(\mathrm{~s}, 1 \mathrm{H}), 7.58\left(\mathrm{dd},{ }^{3} \mathrm{~J}_{\mathrm{HH}}=13.2 \mathrm{~Hz}\right.$, $\left.{ }^{3} J_{\mathrm{HH}}=8.4 \mathrm{~Hz}, 2 \mathrm{H}\right), 7.54\left(\mathrm{~d},{ }^{3} J_{\mathrm{HH}}=6.6 \mathrm{~Hz}, 1 \mathrm{H}\right), 7.49(\mathrm{~s}, 1 \mathrm{H}), 7.37(\mathrm{~m}, 2 \mathrm{H})$, $7.25\left(\mathrm{~d},{ }^{3} J_{\mathrm{HH}}=7.8 \mathrm{~Hz}, 1 \mathrm{H}\right), 3.88\left(\mathrm{~s}, 2 \mathrm{H}, \mathrm{CH}_{2}\right), 2.35\left(\mathrm{~d},{ }^{3} J_{\mathrm{HH}}=3.6 \mathrm{~Hz}, 6 \mathrm{H}\right.$, Me). ${ }^{13}$ C NMR (151 MHz, $\left.\mathrm{CDCl}_{3}, 298 \mathrm{~K}\right): \delta=193.1(\mathrm{C}=\mathrm{O}), 145.6,145.1$, 143.3, 143.2, 141.5, 136.9, 136.8, 130.2, 129.6, 128.1, 127.1, 126.9, 124.6, 124.0, $38.8\left(\mathrm{CH}_{2}\right), 20.1(\mathrm{Me})$, 19.9 (Me). HRMS (ESI) $m / z$ calcd for $\mathrm{C}_{18} \mathrm{H}_{16} \mathrm{NaO}^{+}(\mathrm{M}+\mathrm{Na})^{+}, 271.1093$; found: 271.1057

\section{(2,4-dimethylphenyl)(1H-inden-2-yl)methanone (2s)}

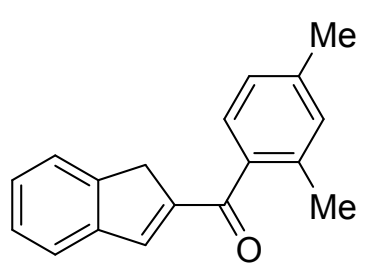

$2 s$ 
$=7.8 \mathrm{~Hz}, 1 \mathrm{H}), 7.30\left(\mathrm{t},{ }^{3} J_{\mathrm{HH}}=7.2 \mathrm{~Hz}, 1 \mathrm{H}\right), 7.26\left(\mathrm{~d},{ }^{3} J_{\mathrm{HH}}=7.8 \mathrm{~Hz}, 2 \mathrm{H}\right), 7.22(\mathrm{~s}, 1 \mathrm{H}), 7.03(\mathrm{~s}, 1 \mathrm{H}), 6.99$ $\left(\mathrm{d},{ }^{3} J_{\mathrm{HH}}=7.8 \mathrm{~Hz}, 1 \mathrm{H}\right), 3.78\left(\mathrm{~s}, 2 \mathrm{H}, \mathrm{CH}_{2}\right), 2.31(\mathrm{~s}, 3 \mathrm{H}, \mathrm{Me}), 2.28(\mathrm{~s}, 3 \mathrm{H}, \mathrm{Me}) .{ }^{13} \mathbf{C} \mathbf{N M R}(151 \mathrm{MHz}$, $\left.\mathrm{CDCl}_{3}, 298 \mathrm{~K}\right): \delta=195.4(\mathrm{C}=\mathrm{O}), 146.8,145.3,144.6,143.0,140.1,136.6,136.4,131.9,128.3,128.3$,

127.0, 125.7, 124.6, 124.0, $37.7\left(\mathrm{CH}_{2}\right), 21.4(\mathrm{Me}), 19.8$ (Me). HRMS (ESI) $m / z$ calcd for $\mathrm{C}_{18} \mathrm{H}_{16} \mathrm{NaO}^{+}$ $(\mathrm{M}+\mathrm{Na})^{+}, 271.1093$; found: 271.1097.

(1H-inden-2-yl)(thiophen-2-yl)methanone (2t)

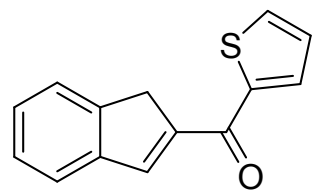

$2 t$

Yield $16.1 \mathrm{mg}, 71 \%$; (petroleum ether/ethyl acetate $=5: 1$ ); Yellow solid; ${ }^{1} \mathbf{H}$ NMR $\left(600 \mathrm{MHz}, \mathrm{CDCl}_{3}, 298 \mathrm{~K}\right): \delta=7.85\left(\mathrm{dd},{ }^{3} J_{\mathrm{HH}}=3.6 \mathrm{~Hz},{ }^{4} J_{\mathrm{HH}}=0.6 \mathrm{~Hz}, 1 \mathrm{H}\right), 7.78(\mathrm{~s}$, $1 \mathrm{H}), 7.67\left(\mathrm{dd},{ }^{3} J_{\mathrm{HH}}=4.8 \mathrm{~Hz},{ }^{4} J_{\mathrm{HH}}=0.6 \mathrm{~Hz}, 1 \mathrm{H}\right), 7.58(\mathrm{~m}, 2 \mathrm{H}), 7.38(\mathrm{~m}, 2 \mathrm{H}), 7.18$ $\left(\mathrm{dd},{ }^{3} J_{\mathrm{HH}}=4.8 \mathrm{~Hz},{ }^{4} J_{\mathrm{HH}}=3.6 \mathrm{~Hz}, 1 \mathrm{H}\right), 3.90\left(\mathrm{~s}, 2 \mathrm{H}, \mathrm{CH}_{2}\right) \cdot{ }^{13} \mathbf{C ~ N M R}(151 \mathrm{MHz}$, $\left.\mathrm{CDCl}_{3}, 298 \mathrm{~K}\right): \delta=183.6(\mathrm{C}=\mathrm{O}), 145.2,144.9,144.1,143.1,141.6,133.0,132.5,128.2,127.9,127.2$, 124.6, 123.9, $39.06\left(\mathrm{CH}_{2}\right)$. HRMS (ESI) $\mathrm{m} / z$ calcd for $\mathrm{C}_{14} \mathrm{H}_{10} \mathrm{NaOS}^{+}(\mathrm{M}+\mathrm{Na})^{+}, 249.0345$; found: 249.0365 .

\section{(5-fluoro-1H-inden-2-yl)(phenyl)methanone (2u)}

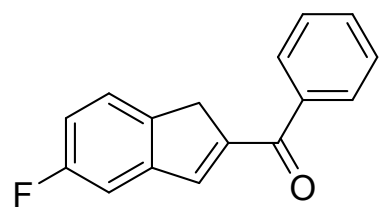

$2 u$

Yield $14.0 \mathrm{mg}, 59 \%$; (petroleum ether/ethyl acetate $=5: 1$ ); Yellow solid; ${ }^{1} \mathbf{H}$ $\operatorname{NMR}\left(600 \mathrm{MHz}, \mathrm{CDCl}_{3}, 298 \mathrm{~K}\right): \delta=7.83\left(\mathrm{t},{ }^{3} J_{\mathrm{HH}}=6.6 \mathrm{~Hz}, 2 \mathrm{H}\right), 7.59\left(\mathrm{dd},{ }^{3} J_{\mathrm{HH}}\right.$ $\left.=13.2 \mathrm{~Hz},{ }^{4} J_{\mathrm{HH}}=7.2 \mathrm{~Hz}, 1 \mathrm{H}\right), 7.49(\mathrm{~m}, 3 \mathrm{H}), 7.45\left(\mathrm{~d},{ }^{3} J_{\mathrm{HH}}=13.2 \mathrm{~Hz}, 1 \mathrm{H}\right), 7.25$ $\left(\mathrm{ddd},{ }^{3} J_{\mathrm{HH}}=8.4 \mathrm{~Hz},{ }^{4} J_{\mathrm{HH}}=1.8 \mathrm{~Hz}, 1 \mathrm{H}\right), 7.07\left(\mathrm{ddd},{ }^{3} J_{\mathrm{HH}}=8.4,{ }^{4} J_{\mathrm{HH}}=2.4 \mathrm{~Hz}\right.$, 1H), $3.88\left(\mathrm{~s}, 2 \mathrm{H}, \mathrm{CH}_{2}\right) .{ }^{13} \mathbf{C}$ NMR (151 MHz, $\left.\mathrm{CDCl}_{3}, 298 \mathrm{~K}\right): \delta=192.6(\mathrm{C}=\mathrm{O}), 162.5,147.3,144.8$, 142.6, 140.3, 138.9, 132.6, 128.9 (2C), 128.4 (2C), 125.2, 114.9, 111.3, $38.5\left(\mathrm{CH}_{2}\right)$. HRMS (ESI) $m / z$ calcd for $\mathrm{C}_{16} \mathrm{H}_{11} \mathrm{FNaO}^{+}(\mathrm{M}+\mathrm{Na})^{+}$, 261.0686; found: 261.0671.

\section{(3-fluoro-1H-inden-2-yl)(phenyl)methanone (2v)}

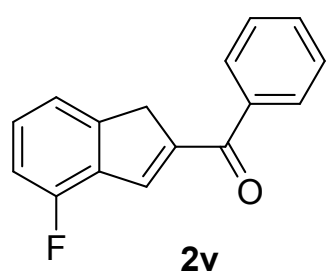

Yield $14.3 \mathrm{mg}, 60 \%$; (petroleum ether/ethyl acetate $=5: 1$ ); Yellow solid; ${ }^{1} \mathbf{H}$ NMR $\left(600 \mathrm{MHz}, \mathrm{CDCl}_{3}, 298 \mathrm{~K}\right): \delta=7.77\left(\mathrm{~d},{ }^{3} J_{\mathrm{HH}}=7.2 \mathrm{~Hz}, 2 \mathrm{H}\right), 7.51(\mathrm{~m}, 1 \mathrm{H}), 7.43(\mathrm{~m}$, 2H), $7.39(\mathrm{~m}, 1 \mathrm{H}), 7.27(\mathrm{~m}, 2 \mathrm{H}), 7.00(\mathrm{~m}, 1 \mathrm{H}), 3.86\left(\mathrm{~m}, 2 \mathrm{H}, \mathrm{CH}_{2}\right) .{ }^{13} \mathbf{C}$ NMR (151 $\left.\mathrm{MHz}, \mathrm{CDCl}_{3}, 298 \mathrm{~K}\right): \delta=192.6(\mathrm{C}=\mathrm{O}), 159.8,158.1,145.2,145.7,142.5,138.7$, 132.2, 129.2, 128.9 (2C), 128.5 (2C), 120.0, 115.0, $35.4\left(\mathrm{CH}_{2}\right)$. HRMS (ESI) $\mathrm{m} / z$ calcd for $\mathrm{C}_{16} \mathrm{H}_{11} \mathrm{FNaO}^{+}$ $(\mathrm{M}+\mathrm{Na})^{+}, 261.0686$; found: 261.0653 . 
(6-methyl-1H-inden-2-yl)(phenyl)methanone (2w)

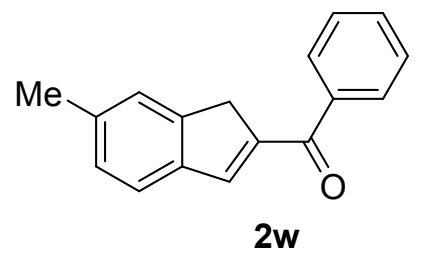

Yield $14.8 \mathrm{mg}$, 63\%; (petroleum ether/ethyl acetate $=5: 1$ ); Yellow solid; ${ }^{1} \mathbf{H}$ $\operatorname{NMR}\left(600 \mathrm{MHz}, \mathrm{CDCl}_{3}, 298 \mathrm{~K}\right): \delta=7.74(\mathrm{~m}, 2 \mathrm{H}), 7.49(\mathrm{~m}, 1 \mathrm{H}), 7.41\left(\mathrm{t},{ }^{3} J_{\mathrm{HH}}\right.$ $=7.2 \mathrm{~Hz}, 2 \mathrm{H}), 7.38(\mathrm{~m}, 1 \mathrm{H}), 7.35\left(\mathrm{~d},{ }^{3} J_{\mathrm{HH}}=7.8 \mathrm{~Hz}, 1 \mathrm{H}\right), 7.29(\mathrm{~s}, 1 \mathrm{H}), 7.11$ (m, 1H), 3.77 (s, 2H, $\left.\mathrm{CH}_{2}\right), 2.35$ (m, 3H, Me). ${ }^{13} \mathbf{C}$ NMR (151 MHz, $\mathrm{CDCl}_{3}$, $298 \mathrm{~K}): \delta=193.0(\mathrm{C}=\mathrm{O}), 145.4,144.1,143.3,142.2,140.5,139.0,136.8,131.9,129.0(2 \mathrm{C}), 128.2(2 \mathrm{C})$, 125.4, 124.1, $38.3\left(\mathrm{CH}_{2}\right), 21.8(\mathrm{Me})$. HRMS (ESI) $\mathrm{m} / z$ calcd for $\mathrm{C}_{17} \mathrm{H}_{14} \mathrm{NaO}^{+}(\mathrm{M}+\mathrm{Na})^{+}, 257.0937$; found: 257.0939 .

(5-methoxy-1H-inden-2-yl)(phenyl)methanone (2x)

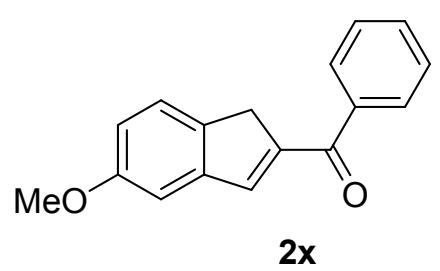

Yield $18.6 \mathrm{mg}, 74 \%$; (petroleum ether/ethyl acetate $=5: 1$ ); Yellow solid; ${ }^{1} \mathbf{H}$ NMR $\left(600 \mathrm{MHz}, \mathrm{CDCl}_{3}, 298 \mathrm{~K}\right): \delta=7.73\left(\mathrm{~d},{ }^{3} \mathrm{~J}_{\mathrm{HH}}=7.2 \mathrm{~Hz}, 2 \mathrm{H}\right), 7.49(\mathrm{t}$, $\left.{ }^{3} J_{\mathrm{HH}}=7.2 \mathrm{~Hz}, 1 \mathrm{H}\right), 7.41\left(\mathrm{t},{ }^{3} J_{\mathrm{HH}}=7.8 \mathrm{~Hz}, 2 \mathrm{H}\right), 7.39(\mathrm{~s}, 1 \mathrm{H}), 7.36\left(\mathrm{~d},{ }^{3} J_{\mathrm{HH}}=\right.$ $8.4 \mathrm{~Hz}, 1 \mathrm{H}), 7.06(\mathrm{~s}, 1 \mathrm{H}), 6.84\left(\mathrm{dd},{ }^{3} J_{\mathrm{HH}}=8.4 \mathrm{~Hz},{ }^{4} J_{\mathrm{HH}}=2.4 \mathrm{~Hz}, 1 \mathrm{H}\right), 3.80$ (s, 3H, OMe), $3.78\left(\mathrm{~s}, 2 \mathrm{H}, \mathrm{CH}_{2}\right) \cdot{ }^{13} \mathbf{C}$ NMR (151 MHz, $\left.\mathrm{CDCl}_{3}, 298 \mathrm{~K}\right): \delta=191.6(\mathrm{C}=\mathrm{O}), 159.7,146.6$, 143.2, 142.2, 138.3, 135.1, 130.6, $127.8(2 \mathrm{C}), 127.3(2 \mathrm{C}), 123.8,112.7,109.0,54.6(\mathrm{OMe}), 37.5\left(\mathrm{CH}_{2}\right)$. HRMS (ESI) $m / z$ calcd for $\mathrm{C}_{17} \mathrm{H}_{14} \mathrm{NaO}_{2}^{+}(\mathrm{M}+\mathrm{Na})^{+}, 273.0886$; found: 273.0880 .

phenyl(5-(trifluoromethyl)-1 $H$-inden-2-yl)methanone (2y)

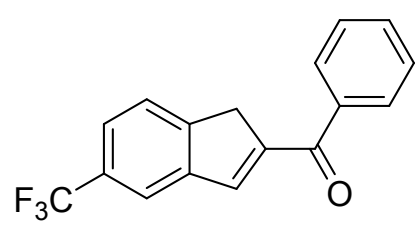

$2 y$

Yield $22.7 \mathrm{mg}, 79 \%$; (petroleum ether/ethyl acetate $=5: 1$ ); Yellow solid; ${ }^{1} \mathbf{H}$ NMR $\left(600 \mathrm{MHz}, \mathrm{CDCl}_{3}, 298 \mathrm{~K}\right): \delta=7.85(\mathrm{~m}, 2 \mathrm{H}), 7.81(\mathrm{~m}, 1 \mathrm{H}), 7.68(\mathrm{~d}$, $\left.{ }^{3} J_{\mathrm{HH}}=7.8 \mathrm{~Hz}, 1 \mathrm{H}\right), 7.64\left(\mathrm{~d},{ }^{3} J_{\mathrm{HH}}=9.0 \mathrm{~Hz}, 1 \mathrm{H}\right), 7.61\left(\mathrm{t},{ }^{3} J_{\mathrm{HH}}=7.8 \mathrm{~Hz}, 1 \mathrm{H}\right)$, $7.52\left(\mathrm{t},{ }^{3} J_{\mathrm{HH}}=7.8 \mathrm{~Hz}, 3 \mathrm{H}\right), 3.96\left(\mathrm{~s}, 2 \mathrm{H}, \mathrm{CH}_{2}\right) .{ }^{13} \mathbf{C ~ N M R}\left(151 \mathrm{MHz}, \mathrm{CDCl}_{3}\right.$, $298 \mathrm{~K}): \delta=192.6(\mathrm{C}=\mathrm{O}), 148.3,147.6,146.7,146.2,143.4,141.9,141.7,138.4,132.4,129.0(2 \mathrm{C}), 128.5$ (2C), 124.8, 123.9, $38.9\left(\mathrm{CH}_{2}\right)$. HRMS (ESI) $m / z$ calcd for $\mathrm{C}_{17} \mathrm{H}_{11} \mathrm{~F}_{3} \mathrm{NaO}^{+}(\mathrm{M}+\mathrm{Na})^{+}, 311.0654$; found: 311.0658 .

(1H-cyclopenta[a]naphthalen-2-yl)(phenyl)methanone (2z)

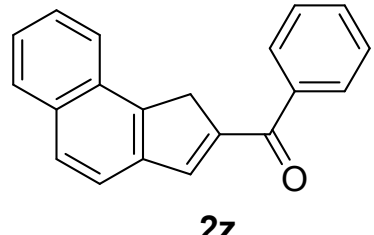

Yield $20.3 \mathrm{mg}$, 75\%; (petroleum ether/ethyl acetate $=5: 1$ ); Yellow solid; ${ }^{1} \mathbf{H}$ $\operatorname{NMR}\left(600 \mathrm{MHz}, \mathrm{CDCl}_{3}, 298 \mathrm{~K}\right): \delta=8.08\left(\mathrm{~d},{ }^{3} \mathrm{~J}_{\mathrm{HH}}=8.4 \mathrm{~Hz}, 1 \mathrm{H}\right), 7.89(\mathrm{~m}, 3 \mathrm{H})$, $7.83\left(\mathrm{~d},{ }^{3} J_{\mathrm{HH}}=8.4 \mathrm{~Hz}, 1 \mathrm{H}\right), 7.63(\mathrm{~m}, 2 \mathrm{H}), 7.58(\mathrm{~m}, 2 \mathrm{H}), 7.52(\mathrm{~m}, 3 \mathrm{H}), 4.25(\mathrm{~s}$, 2H, $\left.\mathrm{CH}_{2}\right) .{ }^{13} \mathbf{C}$ NMR $\left(151 \mathrm{MHz}, \mathrm{CDCl}_{3}, 298 \mathrm{~K}\right): \delta=192.7(\mathrm{C}=\mathrm{O}), 145.0,144.9$, 
143.5, 140.5, 139.3, 133.3, 132.0, 130.2, 129.1, 129.0 (2C), 128.5 (2C), 128.3, 127.1, 126.6, 124.5, 121.8, $37.8\left(\mathrm{CH}_{2}\right)$. HRMS (ESI) $\mathrm{m} / z$ calcd for $\mathrm{C}_{20} \mathrm{H}_{14} \mathrm{NaO}^{+}(\mathrm{M}+\mathrm{Na})^{+}, 293.0937$; found: 293.0926 .

\section{Mechanism Investigation Experiments.}

General Procedure of Cross Experiments : $\mathrm{CoI}_{2}(10 \mathrm{mmol} \%)$ and Ligand (z mmol \%) were added in a $25 \mathrm{~mL}$ pressure tube containing $0.5 \mathrm{~mL}$ degassed anhydrous toluene under argon. The mixture was stirred at room temperature for $15 \mathrm{~min}$. Then In (40 mmol \%) was added and the mixture was stirred for $30 \mathrm{~min}$. To the resulting mixture were added 4 (x mmol), 5 (y mmol) and $i-\mathrm{PrOH}$ (15 equiv.). the mixture was stirred at $80{ }^{\circ} \mathrm{C}$ in a metal bath for $24 \mathrm{~h}$. The solvent was removed in vacuo and the residue was monitored by ${ }^{1} \mathrm{H}$ NMR for yields using $\mathrm{CH}_{2} \mathrm{Br}_{2}$ as the internal standard. 
Table S2: Cross Experiments on the Intermolecular Enone-Aldehyde Coupling. ${ }^{\text {a }}$

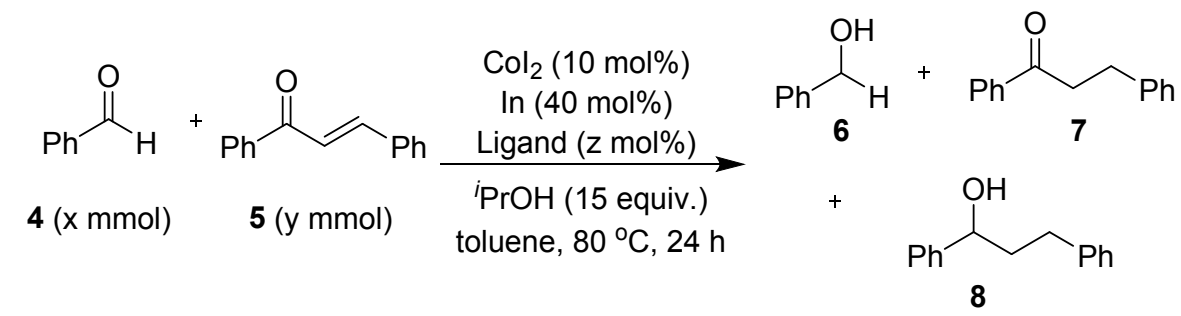

\begin{tabular}{|c|c|c|c|c|c|c|}
\hline \multirow{2}{*}{ Entry } & \multirow{2}{*}{$4(\mathrm{x})$} & \multirow{2}{*}{$5(y)$} & \multirow{2}{*}{ Ligand (z) } & \multicolumn{3}{|c|}{ Yield $(\%)^{b}$} \\
\hline & & & & 6 & 7 & 8 \\
\hline 1 & $(0.1)$ & $(0.1)$ & $(0)$ & 44 & 0 & 0 \\
\hline 2 & $(0.1)$ & $(0.02)$ & $(0)$ & 38 & 0 & 0 \\
\hline 3 & $(0.1)$ & $(0)$ & $(0)$ & 6 & - & - \\
\hline 4 & $(0.1)$ & $(0.1)$ & dtbpx (15) & 58 & 0 & 0 \\
\hline 5 & $(0.1)$ & $(0.02)$ & dtbpx (15) & 8 & 0 & 0 \\
\hline 6 & $(0)$ & $(0.1)$ & $(0)$ & - & 0 & 0 \\
\hline 7 & $(0)$ & $(0.1)$ & dtbpx (15) & - & trace & 0 \\
\hline 8 & $(0)$ & $(0.1)$ & $\mathrm{dppb}(15)$ & - & 17 & 0 \\
\hline 9 & $(0)$ & $(0.1)$ & xtanphos (15) & - & 7 & 0 \\
\hline 10 & $(0)$ & $(0.1)$ & dppp (15) & - & 18 & 0 \\
\hline 11 & $(0)$ & $(0.1)$ & dcpp (15) & - & 14 & 84 \\
\hline 12 & $(0)$ & $(0.1)$ & dcpe (15) & - & 0 & 98 \\
\hline 13 & $(0.1)$ & $(0.1)$ & dcpp (15) & 49 & 95 & 0 \\
\hline${ }^{a}$ React & ondit & oluene & $5 \mathrm{~mL})$, under & Yie & e det & \\
\hline
\end{tabular}

\section{General procedure for deuterium-labeling experiments}

\section{H/D exchange experiments}



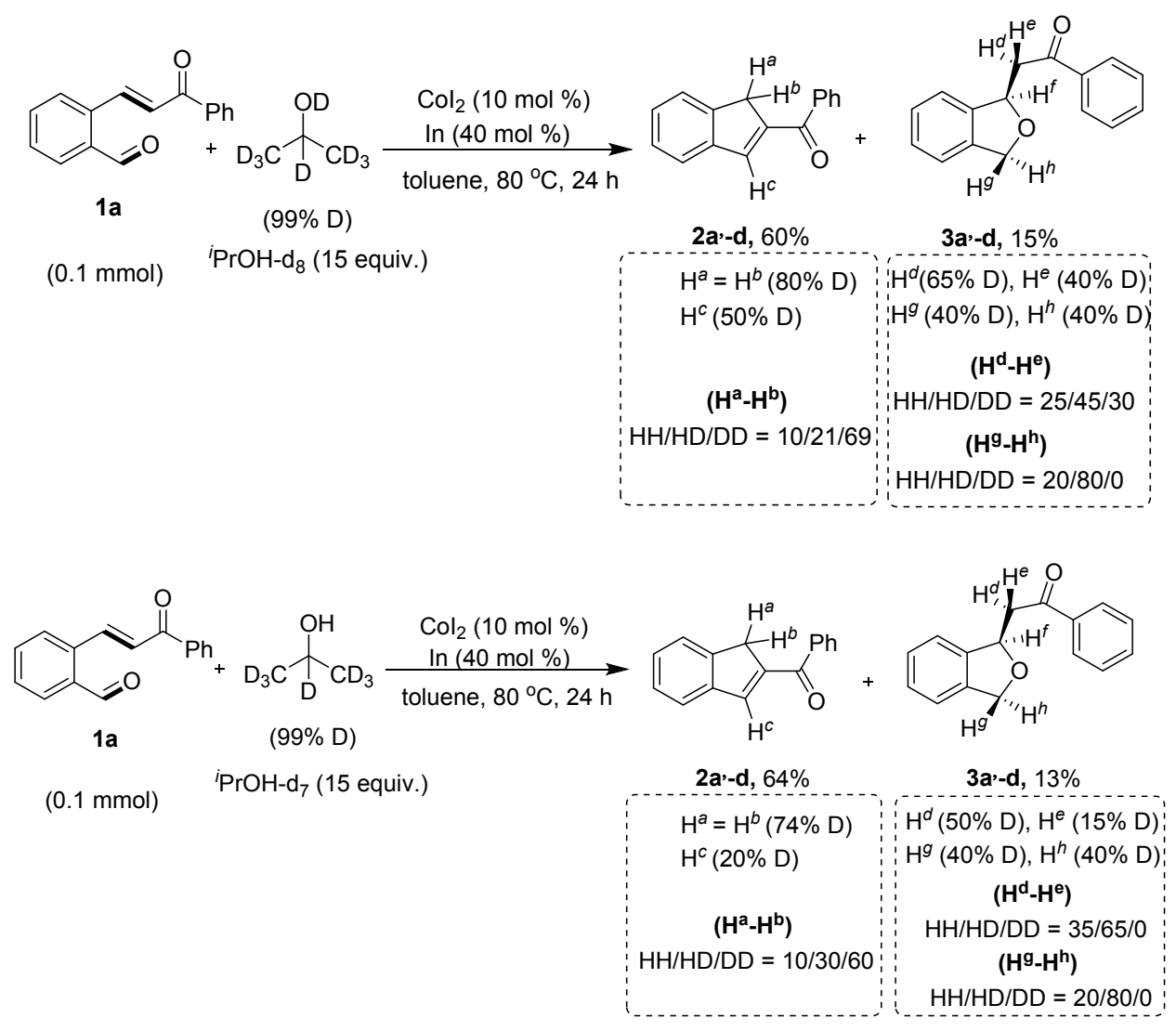

Experiment Procedure : In an argon-filled glove box, 1 a $\left(0.1 \mathrm{mmol}, 1\right.$ equiv.) and $\mathrm{CoI}_{2}(10 \mathrm{~mol} \%)$ were added in a dry flask containing $0.5 \mathrm{~mL}$ degassed anhydrous toluene. The mixture was stirred at room temperature for $15 \mathrm{~min}$. Then the indium powder (40 mol\%) was added and the mixture was stirred for $30 \mathrm{~min}$. To the resulting mixture was added ${ }^{i} \mathrm{PrOH}-\mathrm{d}_{8}$ or ${ }^{i} \mathrm{PrOH}-\mathrm{d}_{7}$ (15 equiv.). The mixture was stirred at $80^{\circ} \mathrm{C}$ in a metal bath for $24 \mathrm{~h}$. After the completion of the reaction, the mixture was concentrated under vacuum and purified by preparative thin layer chromatography (PTLC) with petroleum ether/ethyl acetate to afford the desired product.

\section{Deuterium-Labelling Experiments $\left({ }^{i} \mathrm{PrOH}-\mathrm{d}_{8}\right)$}




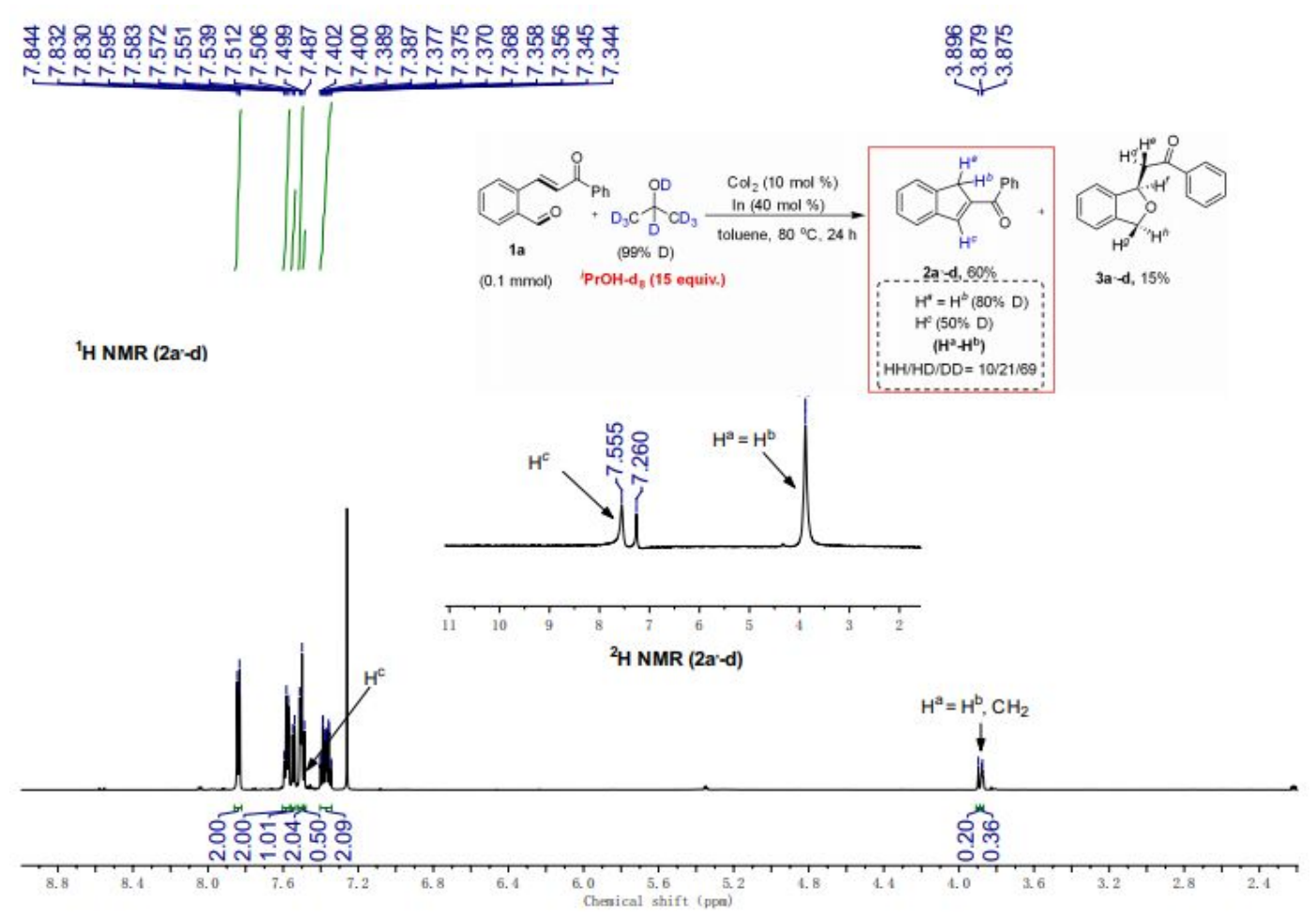

Figure. S1 ${ }^{2} \mathbf{H}$ NMR and ${ }^{\mathbf{1}} \mathbf{H}$ NMR (600 MHz, $\left.\mathrm{CDCl}_{3}, 298 \mathrm{~K}\right)$ of $\mathbf{2} \mathbf{a}$--d $\left({ }^{i} \mathrm{PrOH}-\mathrm{d}_{8}\right)$

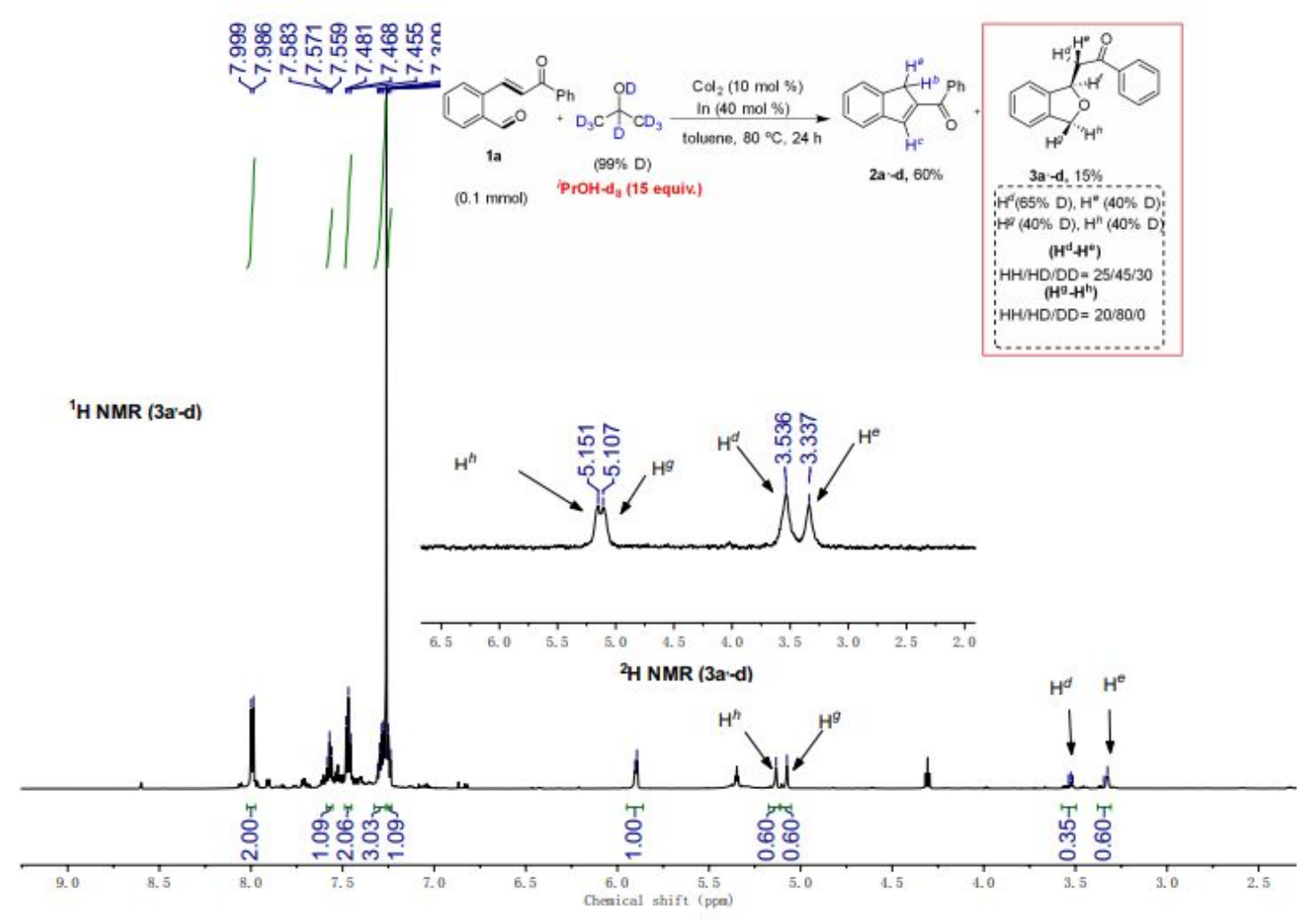

Figure. S2 ${ }^{2} \mathbf{H}$ NMR and ${ }^{\mathbf{1}} \mathbf{H}$ NMR $\left(600 \mathrm{MHz}, \mathrm{CDCl}_{3}, 298 \mathrm{~K}\right)$ of $\mathbf{3 a}-\mathbf{- d}\left({ }^{i} \mathrm{PrOH}-\mathrm{d}_{8}\right)$

\section{Deuterium-Labelling Experiments ( $\left.{ }^{(} \mathrm{PrOH}-\mathrm{d}_{7}\right)$}




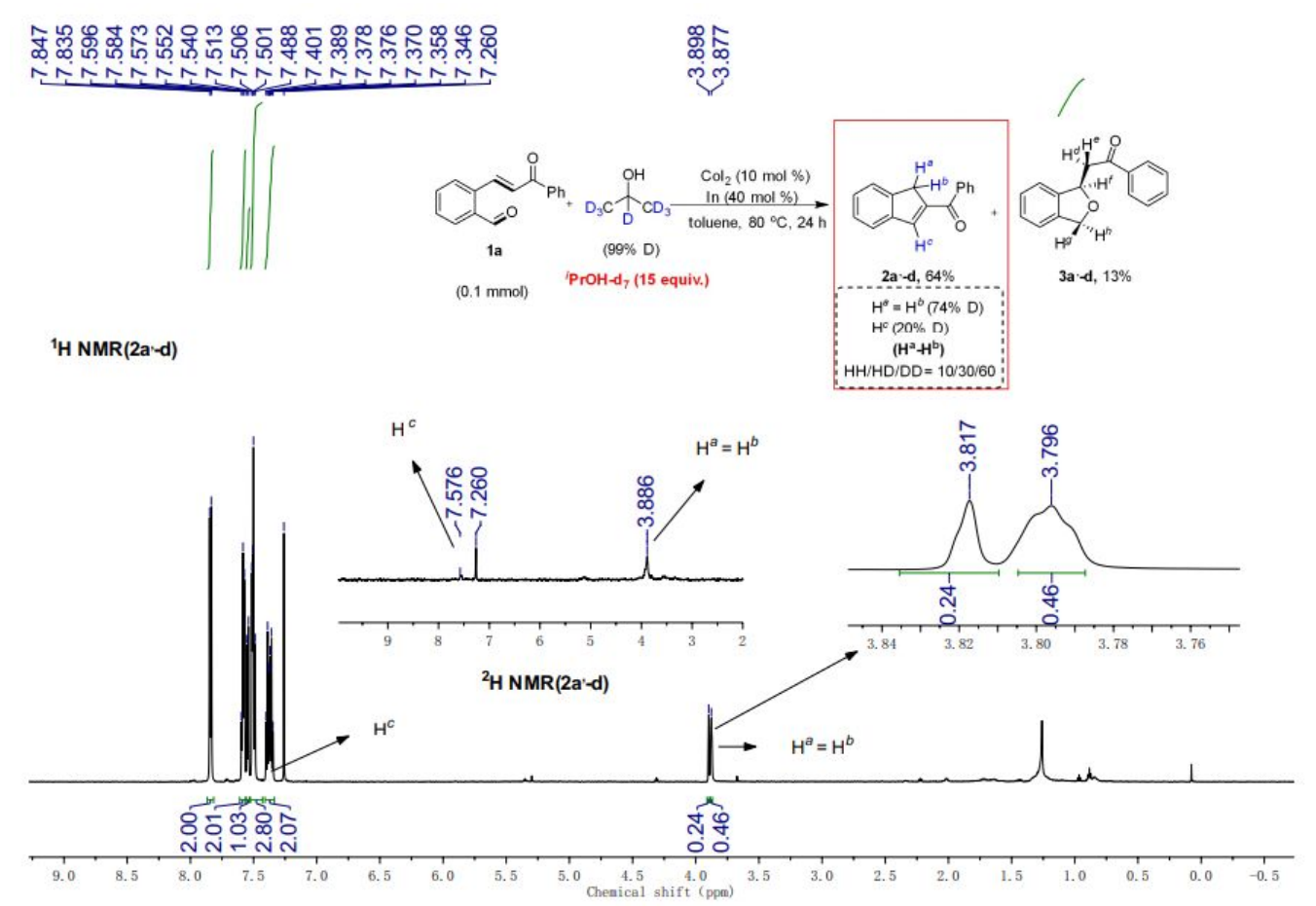

Figure. $\mathbf{S 3}{ }^{2} \mathbf{H}$ NMR and ${ }^{\mathbf{1}} \mathbf{H}$ NMR $\left(600 \mathrm{MHz}, \mathrm{CDCl}_{3}, 298 \mathrm{~K}\right)$ of $\mathbf{2 a}$--d $\left({ }^{i} \mathrm{PrOH}-\mathrm{d}_{7}\right)$

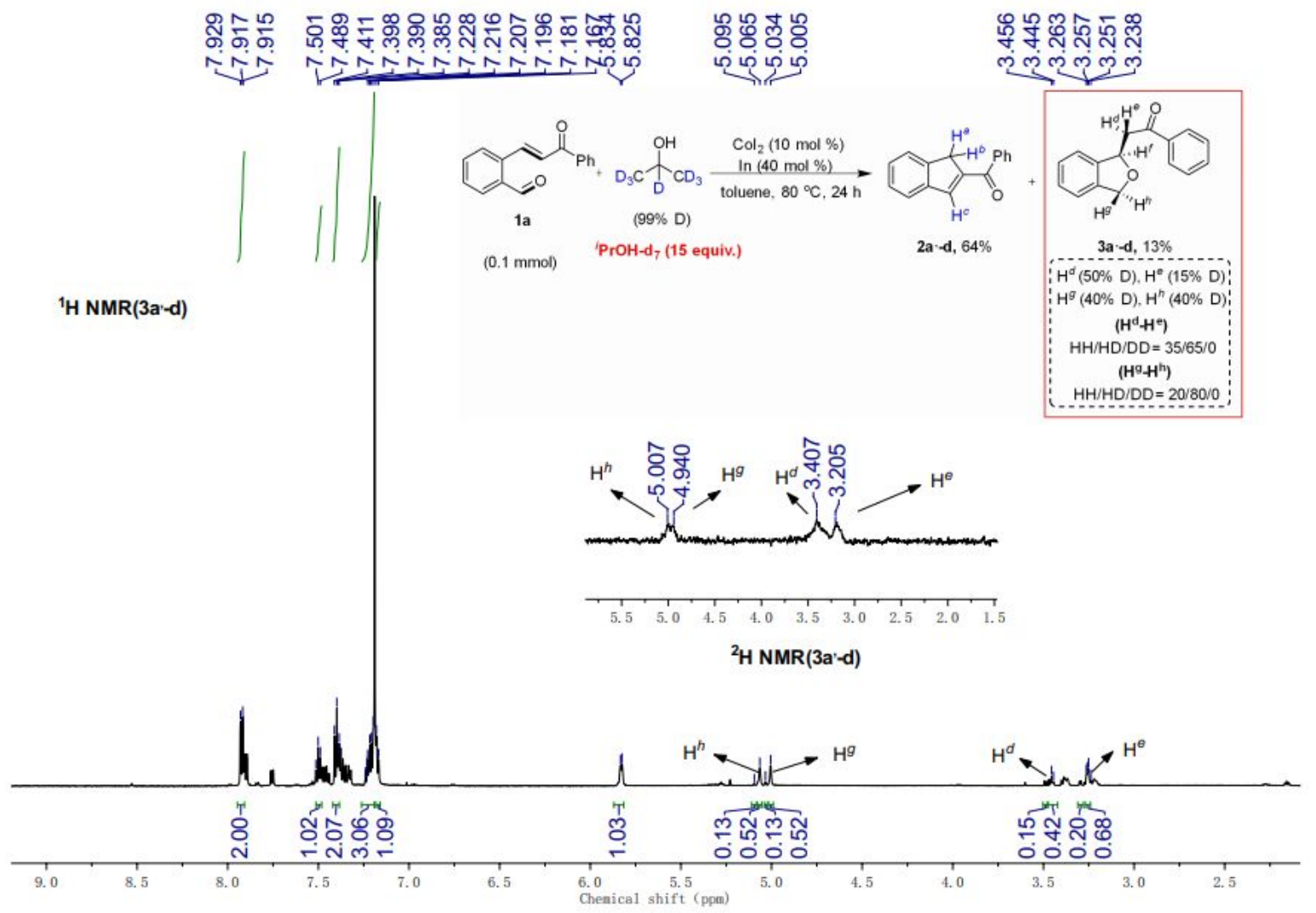

Figure. S4 ${ }^{2} \mathbf{H}$ NMR and ${ }^{1} \mathbf{H}$ NMR $\left(600 \mathrm{MHz}, \mathrm{CDCl}_{3}, 298 \mathrm{~K}\right)$ of $\mathbf{3 a}$--d $\left({ }^{\mathrm{i} P r O H}-\mathrm{d}_{7}\right)$ 
Table S3: Radical-Trapping Experiments<smiles>O=Cc1ccccc1/C=C/C(=O)c1ccccc1</smiles>

1a $(0.1 \mathrm{mmol})$

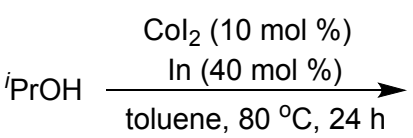

TEMPO (x equiv.)

$\begin{array}{ccccc}\text { Entry } & \begin{array}{c}\text { TEMPO } \\ \text { (x equiv.) }\end{array} & \text { Conv.\% } & \text { Yield }(\%)^{\mathrm{b}} & \mathbf{3 a} \\ 1 & 2.0 & 85 & 25 & 60 \\ 2 & 0.2 & 85 & 46 & 39 \\ 3 & 0.0 & 93 & 83 & 10\end{array}$

$a$ Reaction condition: toluene $(0.5 \mathrm{~mL})$, under Ar. ${ }^{b}$ Conversions and yeilds were determined by ${ }^{1} \mathrm{H}$ NMR using 2-methylindole as the internal standard..

Experiment Procedure : In an argon-filled glove box, $1 \mathrm{a}\left(0.1 \mathrm{mmol}, 1\right.$ equiv.) and $\mathrm{CoI}_{2}(10 \mathrm{~mol} \%)$ were added in a dry flask containing $0.5 \mathrm{~mL}$ degassed anhydrous toluene. The mixture was stirred at room temperature for $15 \mathrm{~min}$. Then the indium powder $(40 \mathrm{~mol} \%)$ was added and the mixture was stirred for $30 \mathrm{~min}$. To the resulting mixture were added ${ }^{i} \mathrm{PrOH}$ (15 equiv.) and Tempo. (x equiv.). The mixture was stirred at $80{ }^{\circ} \mathrm{C}$ in a metal bath for $24 \mathrm{~h}$. After the completion of the reaction, 2methylindole as an internal standard was added and filtered through a short pad of silica gel and concentrated. The residue was monitored by ${ }^{1} \mathrm{H}$ NMR for conversions and yields.

\section{Additional Information}

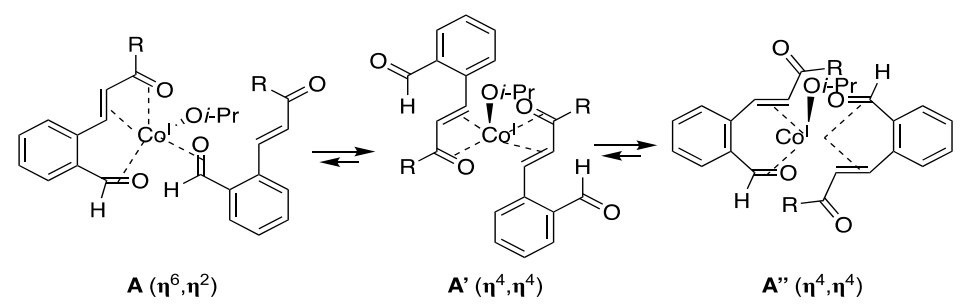

Scheme. S1. Potential Coordination Modes.<smiles>O=Cc1ccccc1CCC(=O)c1ccccc1</smiles>

9a

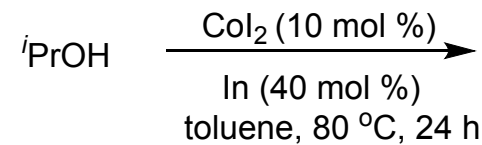

15 equiv.<smiles>O=C(C1=Cc2ccccc2C1)c1ccccc1</smiles>

2a $(33 \%)$

Scheme S2. Control Experiments. 
Experiment Procedure : In an argon-filled glove box, saturated ketone-tethered 9a $(0.1 \mathrm{mmol}, 1$ equiv.) and $\mathrm{CoI}_{2}(10 \mathrm{~mol} \%)$ were added in a dry flask containing $0.5 \mathrm{~mL}$ degassed anhydrous toluene. The mixture was stirred at room temperature for $15 \mathrm{~min}$. Then the indium powder $(40 \mathrm{~mol} \%)$ was added and the mixture was stirred for $30 \mathrm{~min}$. To the resulting mixture were added $i$-PrOH (15 equiv.). The mixture was stirred at $80{ }^{\circ} \mathrm{C}$ in a metal bath for $24 \mathrm{~h}$. After the completion of the reaction, The mixture was concentrated under vacuum and purified by preparative thin layer chromatography (PTLC) with petroleum ether/ethyl acetate (5: 1) to afford the desired product 2a in 33\% yield.

Table S4: Optimization of the lewis basic amines (additives)
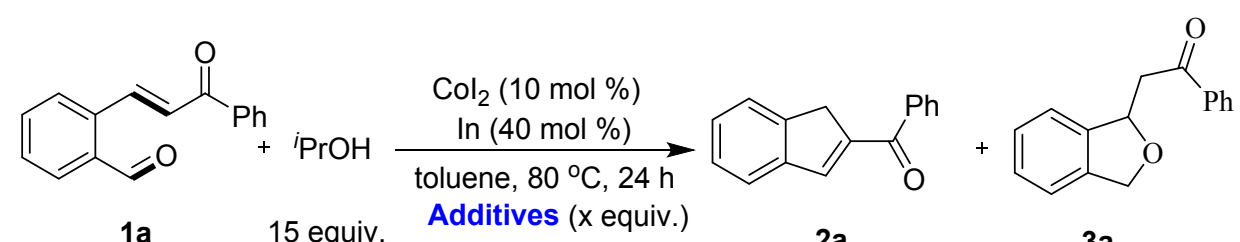

\begin{tabular}{cccc} 
Entry & Additives (x equiv.) & Conv.\% & $\mathbf{2 a : ~} \mathbf{3 a}$ \\
\hline 1 & $\operatorname{NEt}_{3}(2.0)$ & 85 & $1: 2$ \\
2 & $\operatorname{DABCO}(2.0)$ & 82 & $1: 3$ \\
3 & $\operatorname{DBU}(2.0)$ & 76 & $1: 2$ \\
4 & Morpholine (2.0) & 79 & $1: 2$ \\
5 & TEMPO $(0.2)$ & 85 & $5: 4$ \\
6 & TEMPO $(2.0)$ & 85 & $2: 5$ \\
7 & TEMPO (4.0) & $\mathbf{9 2}$ & $\mathbf{1 : 1 0}$ \\
$\mathbf{8}$ & DABCO (4.0) & $\mathbf{9 0}$ & $\mathbf{1 : 9}$
\end{tabular}

${ }^{a}$ Reaction condition: toluene $(0.5 \mathrm{~mL})$, additives (x equiv.), under Ar. ${ }^{b}$ Conversions and yeilds were determined by ${ }^{1} \mathrm{H}$ NMR using 2 -methylindole as the internal standard..

\section{Gram-Scale Reaction}

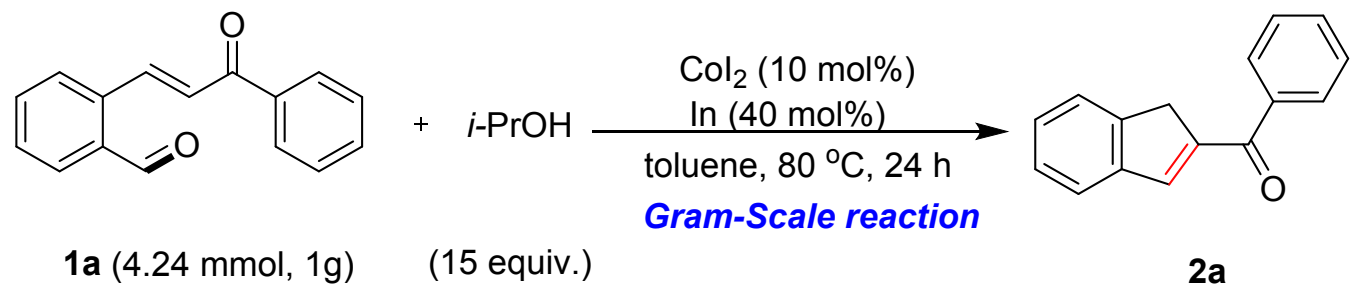

Scheme S3. Gram-scale Preparation of 2a. 
General Procedure : Gram-Scale reaction test was done that a mixture of (E)-2-(3-oxo-3phenylprop-1-en-1-yl)benzaldehyde $1 \mathrm{a}(1 \mathrm{~g}, 4.24 \mathrm{mmol}), \mathrm{CoI}_{2}(0.42 \mathrm{mmol})$, the indium powder $(1.68$ $\mathrm{mmol}), i$-PrOH $(63.60 \mathrm{mmol})$ and toluene $(20 \mathrm{~mL})$ was performed in a $100 \mathrm{~mL}$ pressure tube under argon. The reaction mixture was heated to $80^{\circ} \mathrm{C}$ in an oil bath for 24 hours. After completion of the reaction, the solvent was evaporated to afford the crude product. The residue was purified by column chromatography [silica gel, PE/EtOAc (5:1)] to afford $\mathbf{7 1 \%}$ yield of the desired product $\mathbf{2 a}$

\section{Cobalt-Catalyzed Reductive Enone-Tethered Aldehydes Cyclization with TEMPO.}

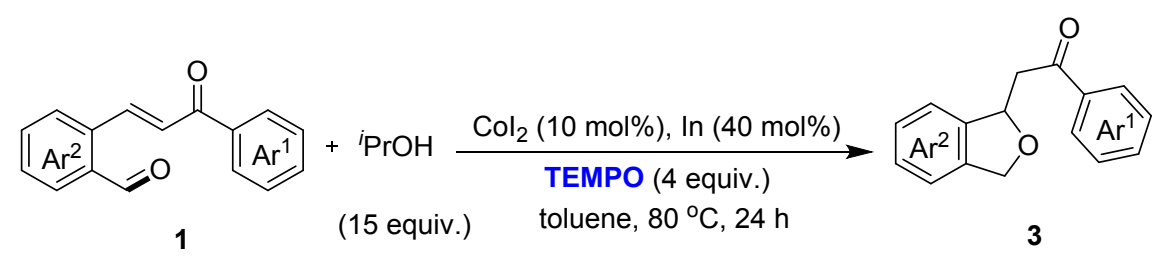

General Procedure : $\mathrm{CoI}_{2}(0.01 \mathrm{mmol})$ and Enone-Tethered Aldehydes $1(0.1 \mathrm{mmol})$ were added in a $25 \mathrm{~mL}$ pressure tube containing $0.5 \mathrm{~mL}$ degassed anhydrous toluene under argon. The mixture was stirred at room temperature for $15 \mathrm{~min}$. Then the indium powder $(0.04 \mathrm{mmol})$ was added and the mixture was stirred for $30 \mathrm{~min}$. To the resulting mixture were added $i$-PrOH (15 equiv) and Tempo. (4 equiv.). The tube was closed and removed from the glove box and the mixture was stirred at $80{ }^{\circ} \mathrm{C}$ in a metal bath for $24 \mathrm{~h}$. After completion of the reaction, the solvent was evaporated to afford the crude product. The residue was purified by preparative thin layer chromatography (PTLC) with petroleum ether/ethyl acetate to afford the desired product.

\section{Analytical Data for Cyclization Products}

\section{2-(1,3-dihydroisobenzofuran-1-yl)-1-phenylethan-1-one (3a)}

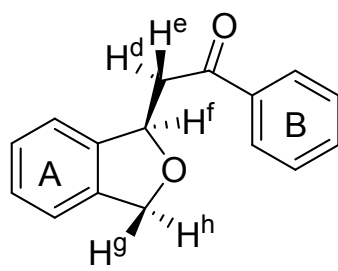

$3 a$

Yield $19.4 \mathrm{mg}, 82 \%$; (petroleum ether/ethyl acetate $=5: 1$ ); Yellow solid; ${ }^{1} \mathbf{H}$ NMR $\left(600 \mathrm{MHz}, \mathrm{CDCl}_{3}, 298 \mathrm{~K}\right): \delta=8.02\left(\mathrm{dm}, 2 \mathrm{H},{ }^{3} J_{\mathrm{HH}}=8.7 \mathrm{~Hz}, o-\mathrm{Ph}^{\mathrm{B}}\right), 7.59(\mathrm{~m}, 1 \mathrm{H}$, $\left.o-\mathrm{Ph}^{\mathrm{B}}\right), 7.49\left(\mathrm{~m}, 2 \mathrm{H}, m-\mathrm{Ph}^{\mathrm{B}}\right), 7.29\left(\mathrm{~m}, 4 \mathrm{H}, \mathrm{Ph}^{\mathrm{A}}\right), 5.93(\mathrm{~m}, 1 \mathrm{H}, \mathrm{CH} f), 5.18\left(\mathrm{dd},{ }^{3} J_{\mathrm{HH}}\right.$ $\left.=12.0 \mathrm{~Hz},{ }^{3} J_{\mathrm{HH}}=2.5 \mathrm{~Hz}, \mathrm{H}^{h}\right), 5.12\left(\mathrm{dm},{ }^{3} J_{\mathrm{HH}}=12.0 \mathrm{~Hz}, \mathrm{H}^{g}\right), 3.57\left(\mathrm{dd},{ }^{2} J_{\mathrm{HH}}=16.6\right.$ 
$\left.\mathrm{Hz},{ }^{3} J_{\mathrm{HH}}=7.3 \mathrm{~Hz}, \mathrm{H}^{d}\right), 3.38\left(\mathrm{dd},{ }^{2} J_{\mathrm{HH}}=16.6 \mathrm{~Hz},{ }^{3} J_{\mathrm{HH}}=5.1 \mathrm{~Hz}, \mathrm{H}^{e}\right) .{ }^{13} \mathbf{C} \mathbf{N M R}\left(151 \mathrm{MHz}, \mathrm{CDCl}_{3}, 298\right.$ $\mathrm{K}): \delta=197.9(\mathrm{C}=\mathrm{O}), 141.6\left(i-\mathrm{Ph}^{\mathrm{A}}\left(\mathrm{CH}_{2}\right)\right), 139.4\left(i-\mathrm{Ph}^{\mathrm{A}}(\mathrm{CH})\right), 137.2\left(i-\mathrm{Ph}^{\mathrm{B}}\right), 133.4\left(p-\mathrm{Ph}^{\mathrm{B}}\right), 128.7(m-$ $\left.\mathrm{Ph}^{\mathrm{B}}\right), 128.4\left(o-\mathrm{Ph}^{\mathrm{B}}\right), 127.9\left(\mathrm{Ph}^{\mathrm{A}}\right), 127.6\left(\mathrm{Ph}^{\mathrm{A}}\right), 121.6\left(\mathrm{Ph}^{\mathrm{A}}\right), 121.1\left(\mathrm{Ph}^{\mathrm{A}}\right), 80.3(\mathrm{CH}), 72.8\left(\mathrm{CH}_{2}\right), 45.7$ $\left(\mathrm{CH}_{2}\right)$. HRMS (ESI) $\mathrm{m} / z$ calcd for $\mathrm{C}_{16} \mathrm{H}_{14} \mathrm{NaO}_{2}{ }^{+}(\mathrm{M}+\mathrm{Na})^{+}, 261.0886$; found: 261.0891 .

\section{2-(1,3-dihydroisobenzofuran-1-yl)-1-(o-tolyl)ethan-1-one (3b)}

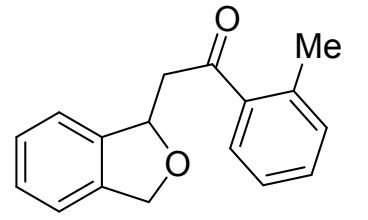

$3 b$

Yield $20.2 \mathrm{mg}, 80 \%$; (petroleum ether/ethyl acetate = 5:1); Yellow solid; ${ }^{1} \mathbf{H}$ NMR $\left(600 \mathrm{MHz}, \mathrm{CDCl}_{3}, 298 \mathrm{~K}\right): \delta=7.60\left(\mathrm{~d},{ }^{3} J_{\mathrm{HH}}=8.4 \mathrm{~Hz}, 1 \mathrm{H}\right), 7.30\left(\mathrm{t},{ }^{3} J_{\mathrm{HH}}=7.8 \mathrm{~Hz}\right.$, 1H), $7.21(\mathrm{~m}, 2 \mathrm{H}), 7.16(\mathrm{~m}, 4 \mathrm{H}), 5.79(\mathrm{~m}, 1 \mathrm{H}), 5.05\left(\mathrm{dd},{ }^{3} J_{\mathrm{HH}}=12.0 \mathrm{~Hz},{ }^{3} J_{\mathrm{HH}}=\right.$ $2.4 \mathrm{~Hz}, 1 \mathrm{H}), 5.02\left(\mathrm{~d},{ }^{3} J_{\mathrm{HH}}=12.0 \mathrm{~Hz}, 1 \mathrm{H}\right), 3.36\left(\mathrm{dd},{ }^{2} J_{\mathrm{HH}}=16.8 \mathrm{~Hz},{ }^{3} J_{\mathrm{HH}}=7.8\right.$

$\mathrm{Hz}, 1 \mathrm{H}), 3.24\left(\mathrm{dd},{ }^{2} J_{\mathrm{HH}}=16.8 \mathrm{~Hz},{ }^{3} J_{\mathrm{HH}}=5.4 \mathrm{~Hz}, 1 \mathrm{H}\right), 2.47(\mathrm{~s}, 3 \mathrm{H}, \mathrm{Me}) .{ }^{13} \mathbf{C} \mathbf{~ N M R}\left(151 \mathrm{MHz}, \mathrm{CDCl}_{3}\right.$, $298 \mathrm{~K}): \delta=201.8(\mathrm{C}=\mathrm{O}), 141.5,139.4,138.6,137.9,132.1,131.6,129.0,127.9,127.6,125.8,121.5$, 121.2, 80.5, 72.8, $48.4\left(\mathrm{CH}_{2}\right), 21.5(\mathrm{Me})$. HRMS (ESI) $m / z$ calcd for $\mathrm{C}_{17} \mathrm{H}_{16} \mathrm{NaO}_{2}{ }^{+}(\mathrm{M}+\mathrm{Na})^{+}, 275.1043$; found: 275.1040 .

\section{2-(1,3-dihydroisobenzofuran-1-yl)-1-(m-tolyl)ethan-1-one (3c)}

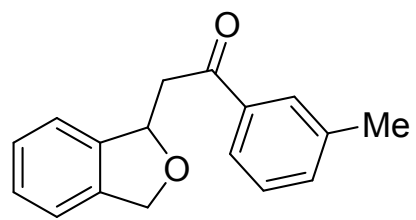

$3 c$

Yield $21.6 \mathrm{mg}, 85 \%$; (petroleum ether/ethyl acetate $=5: 1$ ); Yellow solid; ${ }^{1} \mathbf{H}$ NMR $\left(600 \mathrm{MHz}, \mathrm{CDCl}_{3}, 298 \mathrm{~K}\right): \delta=7.80(\mathrm{~s}, 1 \mathrm{H}), 7.78\left(\mathrm{~d},{ }^{3} J_{\mathrm{HH}}=7.8 \mathrm{~Hz}\right.$, $1 \mathrm{H}), 7.36(\mathrm{~m}, 3 \mathrm{H}), 7.29(\mathrm{~m}, 2 \mathrm{H}), 7.24\left(\mathrm{~d},{ }^{3} J_{\mathrm{HH}}=7.8 \mathrm{~Hz}, 1 \mathrm{H}\right), 5.90(\mathrm{~m}, 1 \mathrm{H})$, $5.15\left(\mathrm{dd},{ }^{3} J_{\mathrm{HH}}=12.0 \mathrm{~Hz},{ }^{3} J_{\mathrm{HH}}=2.4 \mathrm{~Hz}, 1 \mathrm{H}\right), 5.09\left(\mathrm{~d},{ }^{3} J_{\mathrm{HH}}=12.0 \mathrm{~Hz}, 1 \mathrm{H}\right)$, $3.53\left(\mathrm{dd},{ }^{2} J_{\mathrm{HH}}=16.2 \mathrm{~Hz},{ }^{3} J_{\mathrm{HH}}=7.8 \mathrm{~Hz}, 1 \mathrm{H}\right), 3.34\left(\mathrm{dd},{ }^{2} J_{\mathrm{HH}}=16.8 \mathrm{~Hz},{ }^{3} J_{\mathrm{HH}}=4.8 \mathrm{~Hz}, 1 \mathrm{H}\right), 2.47(\mathrm{~s}, 3 \mathrm{H}$, Me). ${ }^{13} \mathbf{C}$ NMR (151 MHz, $\left.\mathrm{CDCl}_{3}, 298 \mathrm{~K}\right): \delta=197.0(\mathrm{C}=\mathrm{O}), 140.5,138.2,137.4,136.1,133.0,127.8$, 127.5, 126.7, 126.4 124.5, 120.5, 119.9, 79.2, 71.6, $44.7\left(\mathrm{CH}_{2}\right), 20.3(\mathrm{Me})$. HRMS (ESI) $\mathrm{m} / z$ calcd for $\mathrm{C}_{17} \mathrm{H}_{16} \mathrm{NaO}_{2}^{+}(\mathrm{M}+\mathrm{H})^{+}, 253.1223$; found: 253.1211.

\section{2-(1,3-dihydroisobenzofuran-1-yl)-1-(p-tolyl)ethan-1-one (3d)}

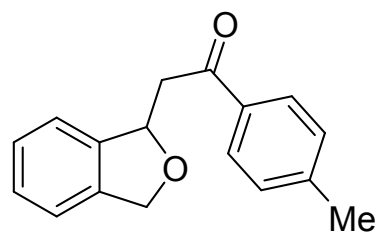

$3 d$

Yield $19.9 \mathrm{mg}, 79 \%$; (petroleum ether/ethyl acetate $=5: 1$ ); Yellow solid; ${ }^{1} \mathbf{H}$ NMR $\left(600 \mathrm{MHz}, \mathrm{CDCl}_{3}, 298 \mathrm{~K}\right): \delta=7.82\left(\mathrm{~d},{ }^{3} \mathrm{~J}_{\mathrm{HH}}=8.4 \mathrm{~Hz}, 2 \mathrm{H}\right), 7.23(\mathrm{~m}, 3 \mathrm{H})$, $7.17\left(\mathrm{t},{ }^{3} J_{\mathrm{HH}}=7.2 \mathrm{~Hz}, 3 \mathrm{H}\right), 5.83(\mathrm{~m}, 1 \mathrm{H}), 5.08\left(\mathrm{dd},{ }^{3} J_{\mathrm{HH}}=12.6 \mathrm{~Hz},{ }^{3} J_{\mathrm{HH}}=1.8\right.$ $\mathrm{Hz}, 1 \mathrm{H}), 5.02\left(\mathrm{~d},{ }^{3} J_{\mathrm{HH}}=12.6 \mathrm{~Hz}, 1 \mathrm{H}\right), 3.45\left(\mathrm{dd},{ }^{2} J_{\mathrm{HH}}=16.8 \mathrm{~Hz},{ }^{3} J_{\mathrm{HH}}=7.2 \mathrm{~Hz}\right.$, $1 \mathrm{H}), 3.25\left(\mathrm{dd},{ }^{2} J_{\mathrm{HH}}=16.8 \mathrm{~Hz},{ }^{3} J_{\mathrm{HH}}=4.8 \mathrm{~Hz}, 1 \mathrm{H}\right), 2.34(\mathrm{~s}, 3 \mathrm{H}, \mathrm{Me}) .{ }^{13} \mathbf{C} \mathbf{N M R}\left(151 \mathrm{MHz}, \mathrm{CDCl}_{3}, 298\right.$ $\mathrm{K}): \delta=196.4(\mathrm{C}=\mathrm{O}), 143.1,140.5,138.3,133.7,128.3(2 \mathrm{C}), 127.4(2 \mathrm{C}), 126.7,126.4,120.5,119.9,79.2$, 
71.6, $44.5\left(\mathrm{CH}_{2}\right), 20.6(\mathrm{Me})$. HRMS (ESI) $\mathrm{m} / z$ calcd for $\mathrm{C}_{17} \mathrm{H}_{16} \mathrm{NaO}_{2}{ }^{+}(\mathrm{M}+\mathrm{H})^{+}, 253.1223$; found: 253.1131 .

\section{1-(4-chlorophenyl)-2-(1,3-dihydroisobenzofuran-1-yl)ethan-1-one (3h)}

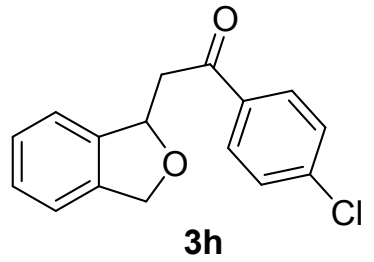

Yield $22.6 \mathrm{mg}, 83 \%$; (petroleum ether/ethyl acetate $=5: 1$ ); Yellow solid; ${ }^{1} \mathbf{H}$ NMR $\left(600 \mathrm{MHz}, \mathrm{CDCl}_{3}, 298 \mathrm{~K}\right): \delta=7.93\left(\mathrm{~d},{ }^{3} J_{\mathrm{HH}}=8.4 \mathrm{~Hz}, 2 \mathrm{H}\right), 7.44\left(\mathrm{~d},{ }^{3} J_{\mathrm{HH}}\right.$ $=8.4 \mathrm{~Hz}, 2 \mathrm{H}), 7.30(\mathrm{~m}, 2 \mathrm{H}), 7.24\left(\mathrm{~d},{ }^{3} J_{\mathrm{HH}}=8.4 \mathrm{~Hz}, 2 \mathrm{H}\right), 5.88(\mathrm{~m}, 1 \mathrm{H}), 5.14(\mathrm{dd}$, $\left.{ }^{3} J_{\mathrm{HH}}=12.0 \mathrm{~Hz},{ }^{3} J_{\mathrm{HH}}=2.4 \mathrm{~Hz}, 1 \mathrm{H}\right), 5.09\left(\mathrm{~d},{ }^{3} J_{\mathrm{HH}}=12.0 \mathrm{~Hz}, 1 \mathrm{H}\right), 3.50\left(\mathrm{dd},{ }^{2} J_{\mathrm{HH}}\right.$ $\left.=16.8 \mathrm{~Hz},{ }^{3} J_{\mathrm{HH}}=7.8 \mathrm{~Hz}, 1 \mathrm{H}\right), 3.31\left(\mathrm{dd},{ }^{2} J_{\mathrm{HH}}=16.8 \mathrm{~Hz},{ }^{3} J_{\mathrm{HH}}=4.8 \mathrm{~Hz}, 1 \mathrm{H}\right) .{ }^{13} \mathbf{C ~ N M R}(151 \mathrm{MHz}$, $\left.\mathrm{CDCl}_{3}, 298 \mathrm{~K}\right): \delta=196.8(\mathrm{C}=\mathrm{O}), 141.3,139.9,139.4,135.6,129.9(2 \mathrm{C}), 129.1(2 \mathrm{C}), 128.0,127.7,121.6$, 121.2, 80.3, 72.8, $45.7\left(\mathrm{CH}_{2}\right)$. HRMS (ESI) $\mathrm{m} / z$ calcd for $\mathrm{C}_{16} \mathrm{H}_{13} \mathrm{ClNaO}_{2}{ }^{+}(\mathrm{M}+\mathrm{Na})^{+}, 295.0496$; found: 295.0517 .

1-(4-bromophenyl)-2-(1,3-dihydroisobenzofuran-1-yl)ethan-1-one (3i)

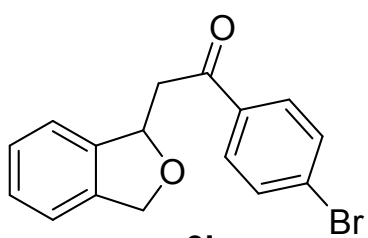

$3 \mathbf{i}$

Yield $23.9 \mathrm{mg}$, 75\%; (petroleum ether/ethyl acetate $=5: 1$ ); Yellow solid; ${ }^{1} \mathbf{H}$ NMR $\left(600 \mathrm{MHz}, \mathrm{CDCl}_{3}, 298 \mathrm{~K}\right): \delta=7.85\left(\mathrm{~d},{ }^{3} J_{\mathrm{HH}}=8.4 \mathrm{~Hz}, 2 \mathrm{H}\right), 7.61\left(\mathrm{~d},{ }^{3} J_{\mathrm{HH}}\right.$ $=8.4 \mathrm{~Hz}, 2 \mathrm{H}), 7.29(\mathrm{~m}, 2 \mathrm{H}), 7.24\left(\mathrm{t},{ }^{3} \mathrm{~J}_{\mathrm{HH}}=7.2 \mathrm{~Hz}, 2 \mathrm{H}\right), 5.87(\mathrm{~m}, 1 \mathrm{H}), 5.14(\mathrm{dd}$, $\left.{ }^{3} J_{\mathrm{HH}}=12.0 \mathrm{~Hz},{ }^{3} J_{\mathrm{HH}}=2.4 \mathrm{~Hz}, 1 \mathrm{H}\right), 5.08\left(\mathrm{~d},{ }^{3} J_{\mathrm{HH}}=12.0 \mathrm{~Hz}, 1 \mathrm{H}\right), 3.49\left(\mathrm{dd},{ }^{2} J_{\mathrm{HH}}\right.$ $\left.=16.2 \mathrm{~Hz},{ }^{3} J_{\mathrm{HH}}=7.8 \mathrm{~Hz}, 1 \mathrm{H}\right), 3.31\left(\mathrm{dd},{ }^{2} J_{\mathrm{HH}}=16.8 \mathrm{~Hz},{ }^{3} J_{\mathrm{HH}}=4.8 \mathrm{~Hz}, 1 \mathrm{H}\right) .{ }^{13} \mathbf{C ~ N M R}(151 \mathrm{MHz}$, $\left.\mathrm{CDCl}_{3}, 298 \mathrm{~K}\right): \delta=197.1(\mathrm{C}=\mathrm{O}), 141.3,139.3,135.9,132.1(2 \mathrm{C}), 129.9(2 \mathrm{C}), 128.7,128.0,127.7,121.6$, 121.2, 80.3, 72.8, $45.7\left(\mathrm{CH}_{2}\right)$. HRMS (ESI) $m / z$ calcd for $\mathrm{C}_{16} \mathrm{H}_{13} \mathrm{BrNaO}_{2}{ }^{+}(\mathrm{M}+\mathrm{Na})^{+}, 338.9991$; found: 338.9983 .

\section{2-(1,3-dihydroisobenzofuran-1-yl)-1-(2-iodophenyl)ethan-1-one (3j)}

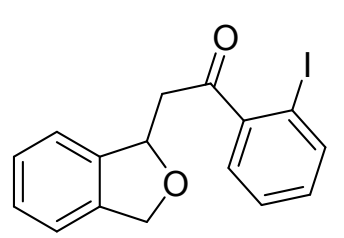

3j

Yield $29.5 \mathrm{mg}, 81 \%$; (petroleum ether/ethyl acetate = 5:1); Yellow solid; ${ }^{1} \mathbf{H}$ NMR $\left(600 \mathrm{MHz}, \mathrm{CDCl}_{3}, 298 \mathrm{~K}\right): \delta=7.92\left(\mathrm{~d},{ }^{3} J_{\mathrm{HH}}=7.8 \mathrm{~Hz}, 1 \mathrm{H}\right), 7.46\left(\mathrm{~d},{ }^{3} J_{\mathrm{HH}}=7.8 \mathrm{~Hz}\right.$, $1 \mathrm{H}), 7.39(\mathrm{~m}, 2 \mathrm{H}), 7.29(\mathrm{~m}, 3 \mathrm{H}), 7.23(\mathrm{~m}, 1 \mathrm{H}), 7.12\left(\mathrm{t},{ }^{3} J_{\mathrm{HH}}=7.8 \mathrm{~Hz}, 1 \mathrm{H}\right), 5.84(\mathrm{~m}$, $1 \mathrm{H}), 5.13\left(\mathrm{dd},{ }^{3} J_{\mathrm{HH}}=12.0 \mathrm{~Hz},{ }^{3} J_{\mathrm{HH}}=2.4 \mathrm{~Hz}, 1 \mathrm{H}\right), 5.08\left(\mathrm{~d},{ }^{3} J_{\mathrm{HH}}=12.0 \mathrm{~Hz}, 1 \mathrm{H}\right)$, $3.39\left(\mathrm{~m}, 2 \mathrm{H}, \mathrm{CH}_{2}\right) .{ }^{13} \mathbf{C}$ NMR $\left(151 \mathrm{MHz}, \mathrm{CDCl}_{3}, 298 \mathrm{~K}\right): \delta=202.2(\mathrm{C}=\mathrm{O}), 144.3,141.1,140.8,139.3$, 131.9, 128.6, 128.2, 127.9, 127.6, 121.6, 121.2, 91.2, 80.3, 72.8, 48.9. HRMS (ESI) $\mathrm{m} / z$ calcd for $\mathrm{C}_{16} \mathrm{H}_{14} \mathrm{IO}_{2}^{+}(\mathrm{M}+\mathrm{H})^{+}, 365.0033$; found: 364.9958 .

2-(1,3-dihydroisobenzofuran-1-yl)-1-(4-iodophenyl)ethan-1-one (3I) 


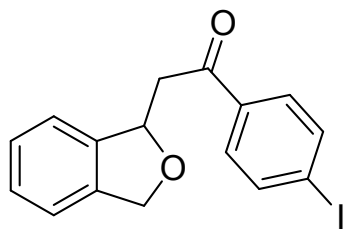

3!

Yield $26.5 \mathrm{mg}, 73 \%$; (petroleum ether/ethyl acetate $=5: 1$ ); Yellow solid; ${ }^{1} \mathbf{H}$ NMR $\left(600 \mathrm{MHz}, \mathrm{CDCl}_{3}, 298 \mathrm{~K}\right): \delta=7.76\left(\mathrm{~d},{ }^{3} J_{\mathrm{HH}}=8.4 \mathrm{~Hz}, 2 \mathrm{H}\right), 7.62\left(\mathrm{~d},{ }^{3} J_{\mathrm{HH}}=8.4\right.$ $\mathrm{Hz}, 2 \mathrm{H}), 7.22(\mathrm{~m}, 2 \mathrm{H}), 7.17\left(\mathrm{t},{ }^{3} J_{\mathrm{HH}}=7.2 \mathrm{~Hz}, 2 \mathrm{H}\right), 5.80(\mathrm{~m}, 1 \mathrm{H}), 5.07\left(\mathrm{dd},{ }^{3} J_{\mathrm{HH}}=\right.$ $\left.12.6 \mathrm{~Hz},{ }^{3} J_{\mathrm{HH}}=2.4 \mathrm{~Hz}, 1 \mathrm{H}\right), 5.01\left(\mathrm{~d},{ }^{3} J_{\mathrm{HH}}=12.0 \mathrm{~Hz}, 1 \mathrm{H}\right), 3.41\left(\mathrm{dd},{ }^{2} J_{\mathrm{HH}}=16.8\right.$ $\left.\mathrm{Hz},{ }^{3} J_{\mathrm{HH}}=7.2 \mathrm{~Hz}, 1 \mathrm{H}\right), 3.23\left(\mathrm{dd},{ }^{2} J_{\mathrm{HH}}=15.6 \mathrm{~Hz},{ }^{3} J_{\mathrm{HH}}=5.4 \mathrm{~Hz}, 1 \mathrm{H}\right) .{ }^{13} \mathbf{C ~ N M R}\left(151 \mathrm{MHz}, \mathrm{CDCl}_{3}, 298\right.$ $\mathrm{K}): \delta=197.2(\mathrm{C}=\mathrm{O}), 141.2,139.2,137.9(2 \mathrm{C}), 136.4,129.7(2 \mathrm{C}), 127.9,127.5,121.4,121.1,101.3,80.1$, 72.7, 45.5. HRMS (ESI) $m / z$ calcd for $\mathrm{C}_{16} \mathrm{H}_{13} \mathrm{INaO}_{2}{ }^{+}(\mathrm{M}+\mathrm{Na})^{+}, 386.9852$; found: 386.9725 .

\section{2-(1,3-dihydroisobenzofuran-1-yl)-1-(4-(trifluoromethyl)phenyl)ethan-1-one (3m)}

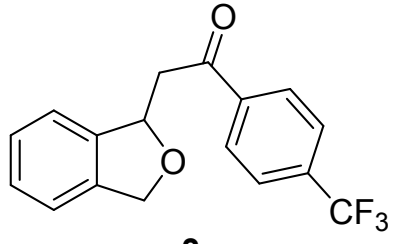

$3 m$

Yield $25.2 \mathrm{mg}, 82 \%$; (petroleum ether/ethyl acetate $=5: 1$ ); Yellow solid; ${ }^{1} \mathbf{H}$ NMR $\left(600 \mathrm{MHz}, \mathrm{CDCl}_{3}, 298 \mathrm{~K}\right): \delta=8.02\left(\mathrm{~d},{ }^{3} J_{\mathrm{HH}}=8.4 \mathrm{~Hz}, 2 \mathrm{H}\right), 7.66\left(\mathrm{~d},{ }^{3} J_{\mathrm{HH}}\right.$ $=8.4 \mathrm{~Hz}, 2 \mathrm{H}), 7.23(\mathrm{~m}, 2 \mathrm{H}), 7.18\left(\mathrm{~d},{ }^{3} J_{\mathrm{HH}}=1.2 \mathrm{~Hz}, 2 \mathrm{H}\right), 5.82(\mathrm{~m}, 1 \mathrm{H}), 5.08$ $\left(\mathrm{dd},{ }^{3} J_{\mathrm{HH}}=12.0 \mathrm{~Hz},{ }^{3} J_{\mathrm{HH}}=2.4 \mathrm{~Hz}, 1 \mathrm{H}\right), 5.02\left(\mathrm{~d},{ }^{3} J_{\mathrm{HH}}=12.0 \mathrm{~Hz}, 1 \mathrm{H}\right), 3.48(\mathrm{dd}$, $\left.{ }^{2} J_{\mathrm{HH}}=16.2 \mathrm{~Hz},{ }^{3} J_{\mathrm{HH}}=7.8 \mathrm{~Hz}, 1 \mathrm{H}\right), 3.30\left(\mathrm{dd},{ }^{2} J_{\mathrm{HH}}=16.8 \mathrm{~Hz},{ }^{3} J_{\mathrm{HH}}=4.8 \mathrm{~Hz}, 1 \mathrm{H}\right) .{ }^{13} \mathbf{C ~ N M R}(151 \mathrm{MHz}$, $\left.\mathrm{CDCl}_{3}, 298 \mathrm{~K}\right): \delta=197.0(\mathrm{C}=\mathrm{O}), 141.1,139.7,139.2,128.7(4 \mathrm{C}), 128.1,127.9,127.6,125.7,121.4$, 121.1, 80.1, 72.6, 45.9. HRMS (ESI) $m / z$ calcd for $\mathrm{C}_{17} \mathrm{H}_{13} \mathrm{~F}_{3} \mathrm{NaO}_{2}{ }^{+}(\mathrm{M}+\mathrm{Na})^{+}, 329.0760$; found: 329.0765 .

\section{2-(1,3-dihydroisobenzofuran-1-yl)-1-(5,6,7,8-tetrahydronaphthalen-2-yl)ethan-1-one (30)}

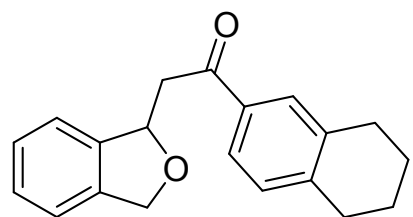

30

Yield $20.1 \mathrm{mg}$, 69\%; (petroleum ether/ethyl acetate = 5:1); Yellow solid; ${ }^{1} \mathbf{H}$ NMR $\left(600 \mathrm{MHz}, \mathrm{CDCl}_{3}, 298 \mathrm{~K}\right): \delta=7.76(\mathrm{~m}, 2 \mathrm{H}), 7.22(\mathrm{~m}, 2 \mathrm{H}), 7.16\left(\mathrm{t},{ }^{3} \mathrm{~J}_{\mathrm{HH}}\right.$ $=7.2 \mathrm{~Hz}, 2 \mathrm{H}), 7.06\left(\mathrm{~d},{ }^{3} J_{\mathrm{HH}}=9.0 \mathrm{~Hz}, 1 \mathrm{H}\right) 5.82(\mathrm{~m}, 1 \mathrm{H}), 5.08\left(\mathrm{dd},{ }^{3} J_{\mathrm{HH}}=12.0\right.$ $\left.\mathrm{Hz},{ }^{3} J_{\mathrm{HH}}=2.4 \mathrm{~Hz}, 1 \mathrm{H}\right), 5.01\left(\mathrm{~d},{ }^{3} J_{\mathrm{HH}}=12.0 \mathrm{~Hz}, 1 \mathrm{H}\right), 3.44\left(\mathrm{dd},{ }^{2} J_{\mathrm{HH}}=16.2\right.$ $\left.\mathrm{Hz},{ }^{3} J_{\mathrm{HH}}=7.2 \mathrm{~Hz}, 1 \mathrm{H}\right), 3.23\left(\mathrm{dd},{ }^{2} J_{\mathrm{HH}}=15.2 \mathrm{~Hz},{ }^{3} J_{\mathrm{HH}}=5.4 \mathrm{~Hz}, 1 \mathrm{H}\right), 2.73(\mathrm{~s}, 4 \mathrm{H}), 1.74(\mathrm{~m}, 4 \mathrm{H}) .{ }^{13} \mathbf{C}$ NMR $\left(151 \mathrm{MHz}, \mathrm{CDCl}_{3}, 298 \mathrm{~K}\right): \delta=197.9(\mathrm{C}=\mathrm{O}), 143.6,141.8,139.4,137.6,134.8,129.5,129.4,127.8$, 127.6, 125.5, 121.7, 121.1, 80.4, 72.7, 45.7, 29.8, 29.5, 23.1, 22.9. HRMS (ESI) $\mathrm{m} / z$ calcd for $\mathrm{C}_{20} \mathrm{H}_{20} \mathrm{NaO}_{2}^{+}(\mathrm{M}+\mathrm{Na})^{+}, 315.1356$; found: 315.1299.

\section{2-(1,3-dihydroisobenzofuran-1-yl)-1-(naphthalen-2-yl)ethan-1-one (3p)}

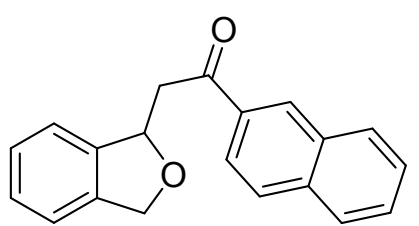

$3 p$
Yield $23.0 \mathrm{mg}$, 80\%; (petroleum ether/ethyl acetate $=5: 1$ ); Yellow solid; ${ }^{1} \mathbf{H}$ NMR $\left(600 \mathrm{MHz}, \mathrm{CDCl}_{3}, 298 \mathrm{~K}\right): \delta=8.42(\mathrm{~s}, 1 \mathrm{H}), 8.01\left(\mathrm{~d},{ }^{3} J_{\mathrm{HH}}=8.4 \mathrm{~Hz}\right.$, $1 \mathrm{H}), 7.87\left(\mathrm{~d},{ }^{3} J_{\mathrm{HH}}=8.4 \mathrm{~Hz}, 1 \mathrm{H}\right), 7.82\left(\mathrm{q},{ }^{3} J_{\mathrm{HH}}=7.2 \mathrm{~Hz}, 2 \mathrm{H}\right), 7.53\left(\mathrm{t},{ }^{3} J_{\mathrm{HH}}=\right.$ $7.2 \mathrm{~Hz}, 1 \mathrm{H}), 7.48\left(\mathrm{t},{ }^{3} J_{\mathrm{HH}}=7.2 \mathrm{~Hz}, 1 \mathrm{H}\right), 7.23(\mathrm{~m}, 2 \mathrm{H}), 7.18(\mathrm{~s}, 2 \mathrm{H}), 5.90(\mathrm{~m}$, 
$1 \mathrm{H}), 5.11\left(\mathrm{dd},{ }^{3} J_{\mathrm{HH}}=12.6 \mathrm{~Hz},{ }^{3} J_{\mathrm{HH}}=2.4 \mathrm{~Hz}, 1 \mathrm{H}\right), 5.04\left(\mathrm{t},{ }^{3} J_{\mathrm{HH}}=12.0 \mathrm{~Hz}, 1 \mathrm{H}\right), 3.62\left(\mathrm{dd},{ }^{2} J_{\mathrm{HH}}=16.2\right.$ $\left.\mathrm{Hz},{ }^{3} J_{\mathrm{HH}}=7.2 \mathrm{~Hz}, 1 \mathrm{H}\right), 3.41\left(\mathrm{t},{ }^{2} J_{\mathrm{HH}}=16.2 \mathrm{~Hz},{ }^{3} J_{\mathrm{HH}}=4.8 \mathrm{~Hz}, 1 \mathrm{H}\right) .{ }^{13} \mathbf{C ~ N M R}\left(151 \mathrm{MHz}, \mathrm{CDCl}_{3}, 298\right.$ $\mathrm{K}): \delta=197.9(\mathrm{C}=\mathrm{O}), 141.6,139.4,135.8,134.6,132.7,130.3,129.8,128.7,128.6,127.9,127.9,127.6$, $126.9,124.1,121.7,121.2,80.4,72.8,45.8$. HRMS (ESI) $m / z$ calcd for $\mathrm{C}_{20} \mathrm{H}_{16} \mathrm{NaO}_{2}{ }^{+}(\mathrm{M}+\mathrm{Na})^{+}, 311.1043$; found: 311.1056 .

\section{2-(1,3-dihydroisobenzofuran-1-yl)-1-(3,4-dimethoxyphenyl)ethan-1-one (3q)}

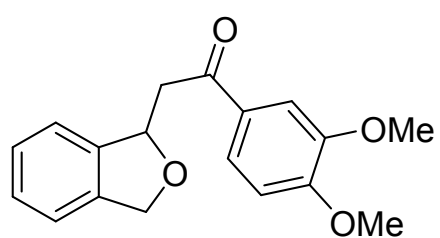

$3 q$

Yield $22.2 \mathrm{mg}, 75 \%$; (petroleum ether/ethyl acetate = 5:1); Yellow solid; ${ }^{1} \mathbf{H}$ NMR (600 MHz, $\left.\mathrm{CDCl}_{3}, 298 \mathrm{~K}\right): \delta=7.52(\mathrm{~m}, 2 \mathrm{H}), 7.22(\mathrm{~m}, 3 \mathrm{H}), 7.17(\mathrm{t}$, $\left.{ }^{3} J_{\mathrm{HH}}=7.2 \mathrm{~Hz}, 1 \mathrm{H}\right), 6.81\left(\mathrm{~d},{ }^{3} J_{\mathrm{HH}}=7.8 \mathrm{~Hz}, 1 \mathrm{H}\right) 5.82(\mathrm{~m}, 1 \mathrm{H}), 5.08\left(\mathrm{dd},{ }^{3} J_{\mathrm{HH}}\right.$ $\left.=12.6 \mathrm{~Hz},{ }^{3} J_{\mathrm{HH}}=2.4 \mathrm{~Hz}, 1 \mathrm{H}\right), 5.02\left(\mathrm{~d},{ }^{3} J_{\mathrm{HH}}=12.0 \mathrm{~Hz}, 1 \mathrm{H}\right), 3.87\left(\mathrm{~d},{ }^{3} J_{\mathrm{HH}}=\right.$ $3.0 \mathrm{~Hz}, 6 \mathrm{H}, \mathrm{OMe}), 3.44\left(\mathrm{dd},{ }^{2} J_{\mathrm{HH}}=16.8 \mathrm{~Hz},{ }^{3} J_{\mathrm{HH}}=7.2 \mathrm{~Hz}, 1 \mathrm{H}\right), 3.23\left(\mathrm{dd},{ }^{2} J_{\mathrm{HH}}=15.2 \mathrm{~Hz},{ }^{3} J_{\mathrm{HH}}=5.4\right.$ $\mathrm{Hz}, 1 \mathrm{H}) .{ }^{13} \mathbf{C}$ NMR $\left(151 \mathrm{MHz}, \mathrm{CDCl}_{3}, 298 \mathrm{~K}\right): \delta=196.4(\mathrm{C}=\mathrm{O}), 153.5,149.0,141.5,139.3,130.4,127.8$, 127.5, 123.2, 121.6, 121.0, 110.4, 110.0, 80.5, 72.6, 56.1 (OMe), $45.2\left(\mathrm{CH}_{2}\right)$. HRMS (ESI) $\mathrm{m} / z$ calcd for $\mathrm{C}_{18} \mathrm{H}_{18} \mathrm{NaO}_{4}{ }^{+}(\mathrm{M}+\mathrm{Na})^{+}, 321.1097$; found: 321.1069 .

\section{2-(1,3-dihydroisobenzofuran-1-yl)-1-(thiophen-2-yl)ethan-1-one (3t)}

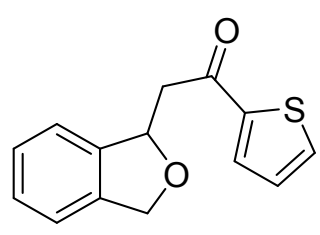

$3 \mathbf{t}$

Yield $15.9 \mathrm{mg}, 65 \%$; (petroleum ether/ethyl acetate $=5: 1$ ); Yellow solid; ${ }^{1} \mathbf{H}$ NMR $\left(600 \mathrm{MHz}, \mathrm{CDCl}_{3}, 298 \mathrm{~K}\right): \delta=7.73\left(\mathrm{~d},{ }^{3} J_{\mathrm{HH}}=3.0 \mathrm{~Hz}, 1 \mathrm{H}\right), 7.66\left(\mathrm{~d},{ }^{3} J_{\mathrm{HH}}=4.2 \mathrm{~Hz}\right.$, 1H), $7.29(\mathrm{~m}, 2 \mathrm{H}), 7.24(\mathrm{~m}, 2 \mathrm{H}), 7.13\left(\mathrm{t},{ }^{3} J_{\mathrm{HH}}=4.8 \mathrm{~Hz}, 1 \mathrm{H}\right), 5.87(\mathrm{~m}, 1 \mathrm{H}), 5.16(\mathrm{dd}$, $\left.{ }^{3} J_{\mathrm{HH}}=12.0 \mathrm{~Hz},{ }^{3} J_{\mathrm{HH}}=2.4 \mathrm{~Hz}, 1 \mathrm{H}\right), 5.09\left(\mathrm{~d},{ }^{3} J_{\mathrm{HH}}=12.0 \mathrm{~Hz}, 1 \mathrm{H}\right), 3.46\left(\mathrm{dd},{ }^{2} J_{\mathrm{HH}}=\right.$ $\left.15.6 \mathrm{~Hz},{ }^{3} J_{\mathrm{HH}}=7.8 \mathrm{~Hz}, 1 \mathrm{H}\right), 3.27\left(\mathrm{dd},{ }^{2} J_{\mathrm{HH}}=15.6 \mathrm{~Hz},{ }^{3} J_{\mathrm{HH}}=4.8 \mathrm{~Hz}, 1 \mathrm{H}\right) .{ }^{13} \mathbf{C} \mathbf{~ N M R}\left(151 \mathrm{MHz}, \mathrm{CDCl}_{3}\right.$, $298 \mathrm{~K}): \delta=190.7(\mathrm{C}=\mathrm{O}), 144.7,141.3,139.3,134.2,132.7,128.3,127.9,127.6,121.6,121.2,80.5,72.8$, 46.5. HRMS (ESI) $m / z$ calcd for $\mathrm{C}_{14} \mathrm{H}_{13} \mathrm{O}_{2} \mathrm{~S}^{+}(\mathrm{M}+\mathrm{H})^{+}, 245.0631$; found: 245.0588 .

\section{2-(5-methoxy-1,3-dihydroisobenzofuran-1-yl)-1-phenylethan-1-one (3x)}

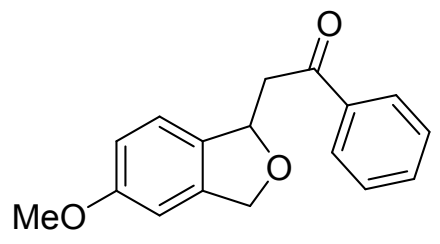

$3 \mathbf{x}$

Yield $18.8 \mathrm{mg}, 70 \%$; (petroleum ether/ethyl acetate $=5: 1$ ); Yellow solid; ${ }^{1} \mathbf{H}$ NMR (600 MHz, $\left.\mathrm{CDCl}_{3}, 298 \mathrm{~K}\right): \delta=7.92\left(\mathrm{~d},{ }^{3} J_{\mathrm{HH}}=8.4 \mathrm{~Hz}, 2 \mathrm{H}\right), 7.50(\mathrm{~m}$, $1 \mathrm{H}), 7.39\left(\mathrm{t},{ }^{3} J_{\mathrm{HH}}=7.8 \mathrm{~Hz}, 2 \mathrm{H}\right), 7.08\left(\mathrm{~d},{ }^{3} J_{\mathrm{HH}}=8.4 \mathrm{~Hz}, 1 \mathrm{H}\right), 6.74\left(\mathrm{~d},{ }^{3} J_{\mathrm{HH}}=\right.$ $8.4 \mathrm{~Hz}, 1 \mathrm{H}), 6.69\left(\mathrm{~d},{ }^{3} J_{\mathrm{HH}}=1.8 \mathrm{~Hz}, 1 \mathrm{H}\right), 5.77(\mathrm{~m}, 1 \mathrm{H}), 5.04\left(\mathrm{dd},{ }^{3} J_{\mathrm{HH}}=12.6\right.$ $\left.\mathrm{Hz},{ }^{3} J_{\mathrm{HH}}=2.4 \mathrm{~Hz}, 1 \mathrm{H}\right), 4.98\left(\mathrm{~d},{ }^{3} J_{\mathrm{HH}}=12.0 \mathrm{~Hz}, 1 \mathrm{H}\right), 3.74(\mathrm{~s}, 3 \mathrm{H}, \mathrm{OMe}), 3.44\left(\mathrm{dd},{ }^{2} J_{\mathrm{HH}}=15.8 \mathrm{~Hz},{ }^{3} J_{\mathrm{HH}}\right.$ $=7.2 \mathrm{~Hz}, 1 \mathrm{H}), 3.25\left(\mathrm{dd},{ }^{2} J_{\mathrm{HH}}=15.6 \mathrm{~Hz},{ }^{3} J_{\mathrm{HH}}=5.4 \mathrm{~Hz}, 1 \mathrm{H}\right) \cdot{ }^{13} \mathbf{C ~ N M R}\left(151 \mathrm{MHz}, \mathrm{CDCl}_{3}, 298 \mathrm{~K}\right): \delta=$ 
$196.9(\mathrm{C}=\mathrm{O}), 158.8,139.9,136.1,132.5,132.2,127.6,127.3,121.3,112.6,105.2,78.9(\mathrm{CHOH}), 71.5$, $54.5(\mathrm{OMe}), 44.9\left(\mathrm{CH}_{2}\right)$. HRMS (ESI) $\mathrm{m} / z$ calcd for $\mathrm{C}_{17} \mathrm{H}_{16} \mathrm{NaO}_{3}{ }^{+}(\mathrm{M}+\mathrm{Na})^{+}$, 291.0992; found: 291.0787 . 1-phenyl-2-(5-(trifluoromethyl)-1,3-dihydroisobenzofuran-1-yl)ethan-1-one (3y)

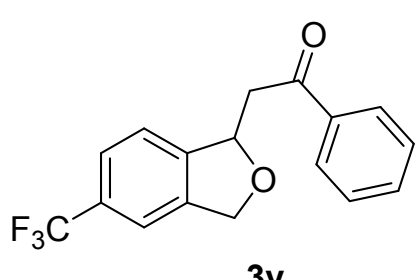

$3 y$ Yield $26.6 \mathrm{mg}, 87 \%$; (petroleum ether/ethyl acetate = 5:1); Yellow solid; ${ }^{1} \mathbf{H}$ NMR $\left(600 \mathrm{MHz}, \mathrm{CDCl}_{3}, 298 \mathrm{~K}\right): \delta=7.91\left(\mathrm{~d},{ }^{3} J_{\mathrm{HH}}=7.8 \mathrm{~Hz}, 2 \mathrm{H}\right), 7.52(\mathrm{t}$, $\left.{ }^{3} J_{\mathrm{HH}}=7.8 \mathrm{~Hz}, 1 \mathrm{H}\right), 7.47\left(\mathrm{t},{ }^{3} J_{\mathrm{HH}}=8.4 \mathrm{~Hz}, 2 \mathrm{H}\right), 7.41(\mathrm{~m}, 3 \mathrm{H}), 7.32\left(\mathrm{~d},{ }^{3} J_{\mathrm{HH}}=\right.$ $7.8 \mathrm{~Hz}, 1 \mathrm{H}), 5.87(\mathrm{~m}, 1 \mathrm{H}), 5.12\left(\mathrm{dd},{ }^{3} J_{\mathrm{HH}}=12.6 \mathrm{~Hz},{ }^{3} \mathrm{~J}_{\mathrm{HH}}=2.4 \mathrm{~Hz}, 1 \mathrm{H}\right), 5.05$ $\left(\mathrm{d},{ }^{3} J_{\mathrm{HH}}=12.6 \mathrm{~Hz}, 1 \mathrm{H}\right), 3.52\left(\mathrm{dd},{ }^{2} J_{\mathrm{HH}}=16.8 \mathrm{~Hz},{ }^{3} J_{\mathrm{HH}}=6.6 \mathrm{~Hz}, 1 \mathrm{H}\right), 3.30\left(\mathrm{dd},{ }^{2} J_{\mathrm{HH}}=16.8 \mathrm{~Hz},{ }^{3} J_{\mathrm{HH}}=\right.$ $6.0 \mathrm{~Hz}, 1 \mathrm{H}) .{ }^{13} \mathrm{C}$ NMR $\left(151 \mathrm{MHz}, \mathrm{CDCl}_{3}, 298 \mathrm{~K}\right): \delta=197.5(\mathrm{C}=\mathrm{O}), 145.7,140.4,136.9,133.6,128.9$ (2C), 128.4 (2C), 127.7, 125.0, 122.3, 118.4, 111.7, 80.1, 72.5, $45.3\left(\mathrm{CH}_{2}\right)$. HRMS (ESI) $\mathrm{m} / z$ calcd for $\mathrm{C}_{17} \mathrm{H}_{13} \mathrm{~F}_{3} \mathrm{NaO}_{2}^{+}(\mathrm{M}+\mathrm{Na})^{+}, 329.0760$; found: 329.0737 . 


\section{VIII. ${ }^{1} \mathrm{H}$ and ${ }^{13} \mathrm{C}$ NMR Spectra}

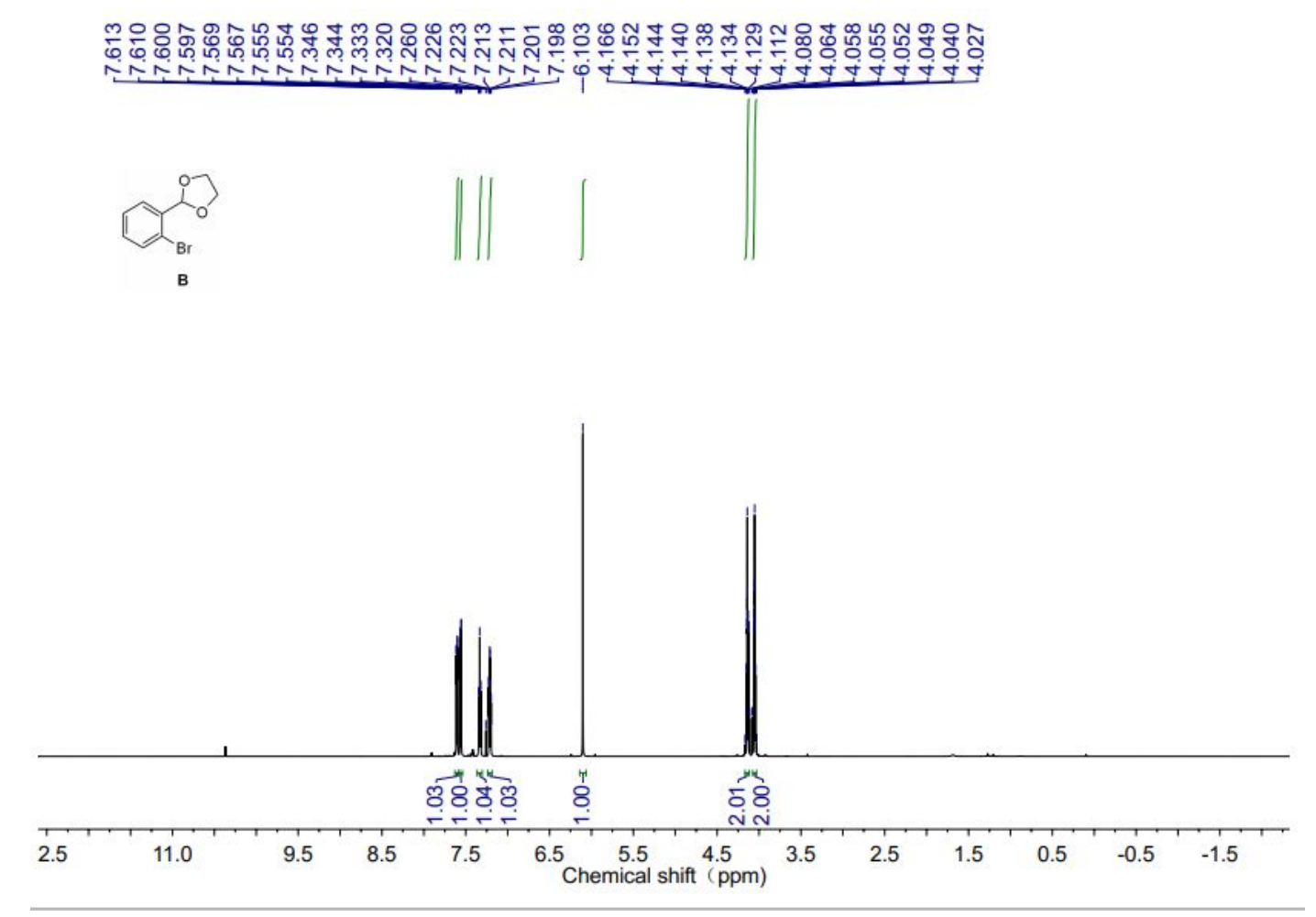

${ }^{\mathbf{1}} \mathbf{H}$ NMR $\left(600 \mathrm{MHz}, \mathrm{CDCl}_{3}, 298 \mathrm{~K}\right)$ of $\mathbf{B}$
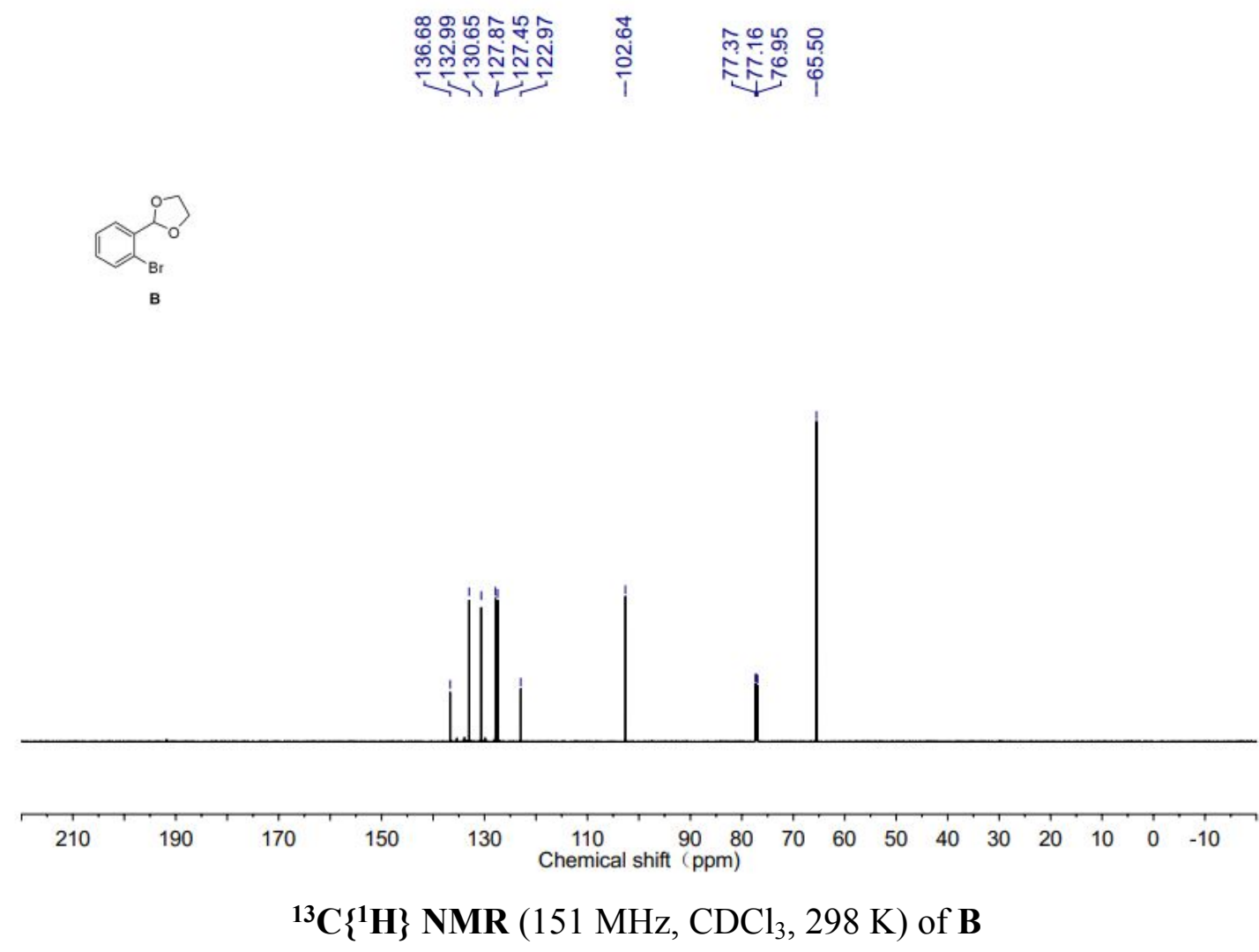

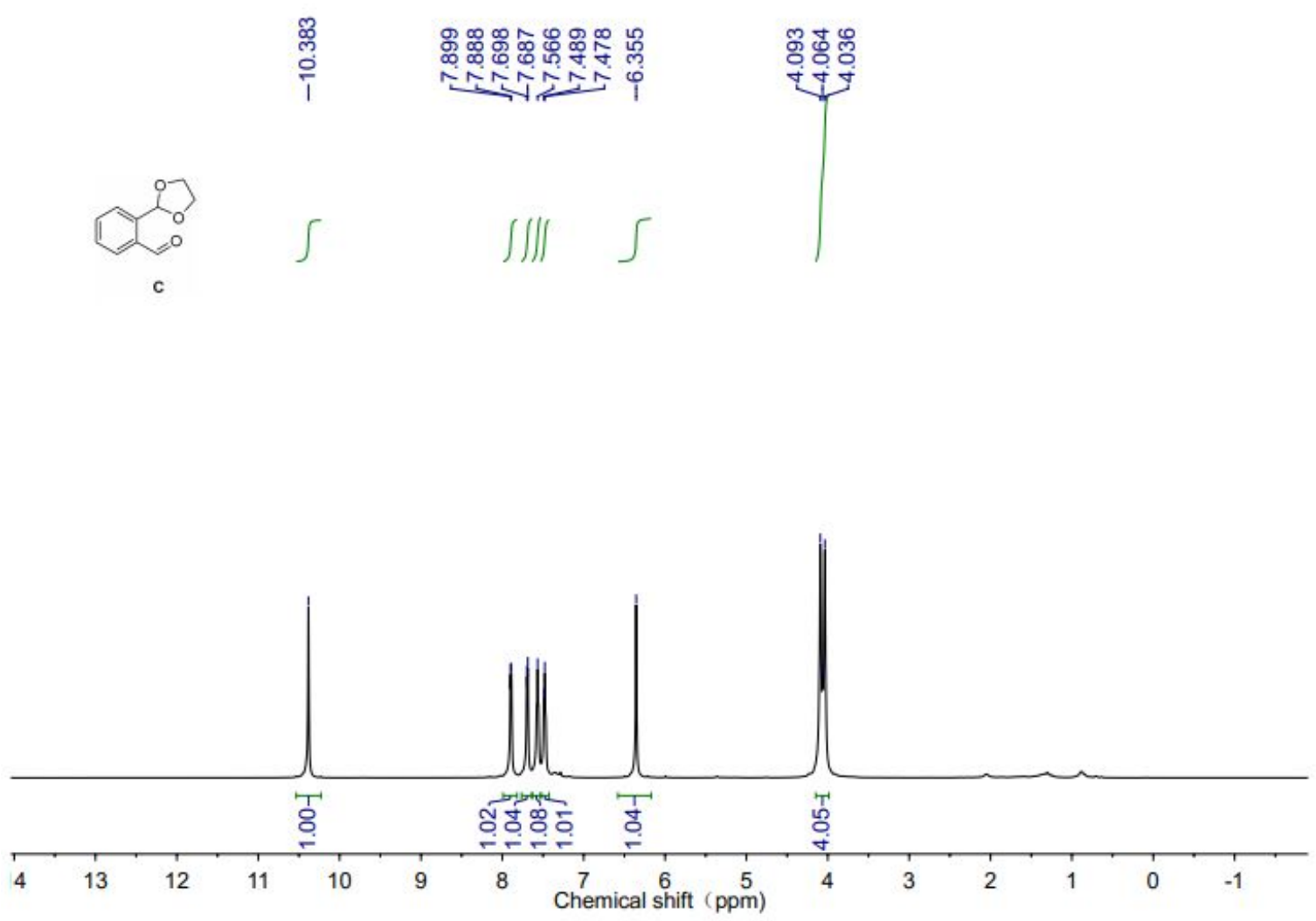

${ }^{1} \mathbf{H}$ NMR $\left(600 \mathrm{MHz}, \mathrm{CDCl}_{3}, 298 \mathrm{~K}\right)$ of $\mathbf{C}$

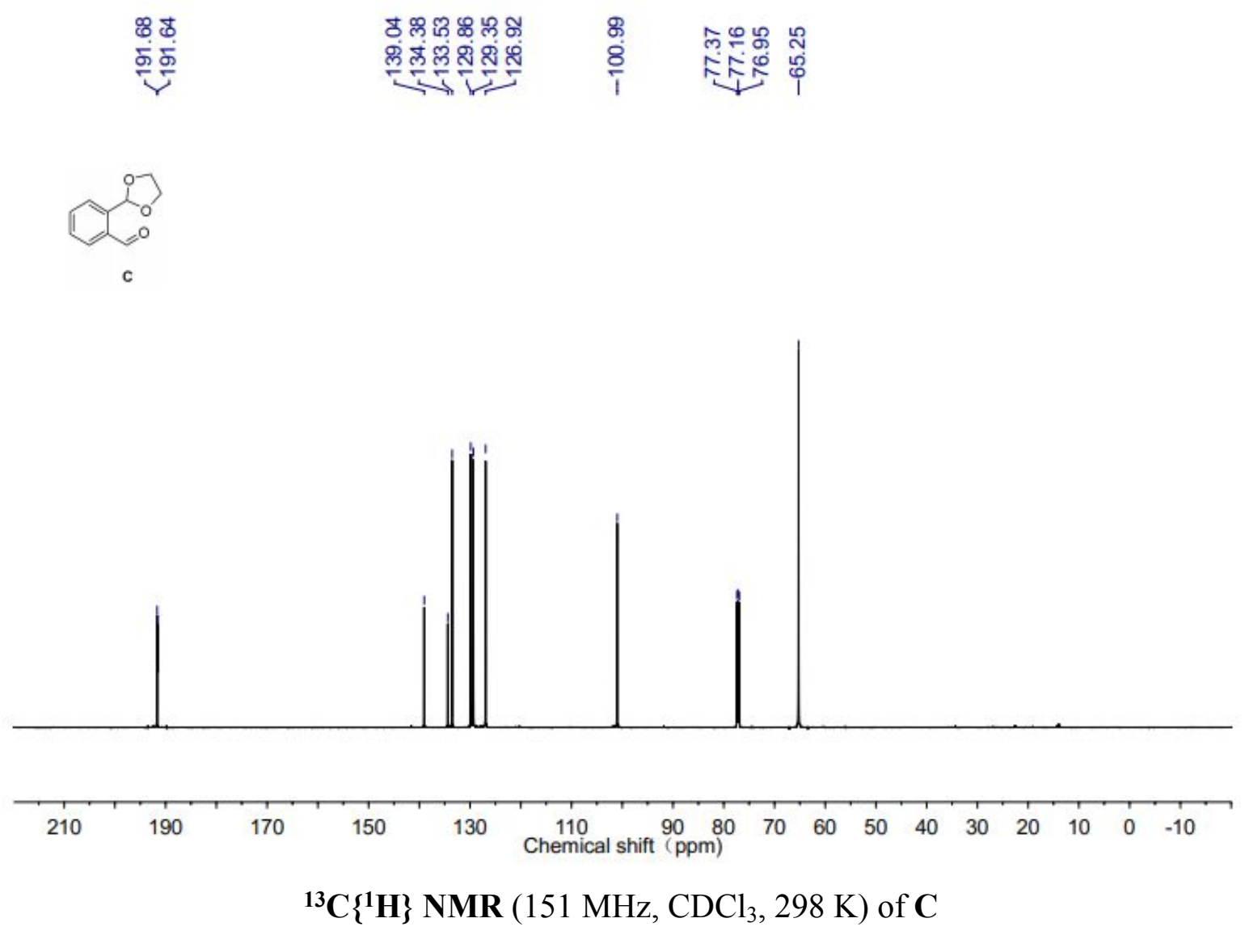



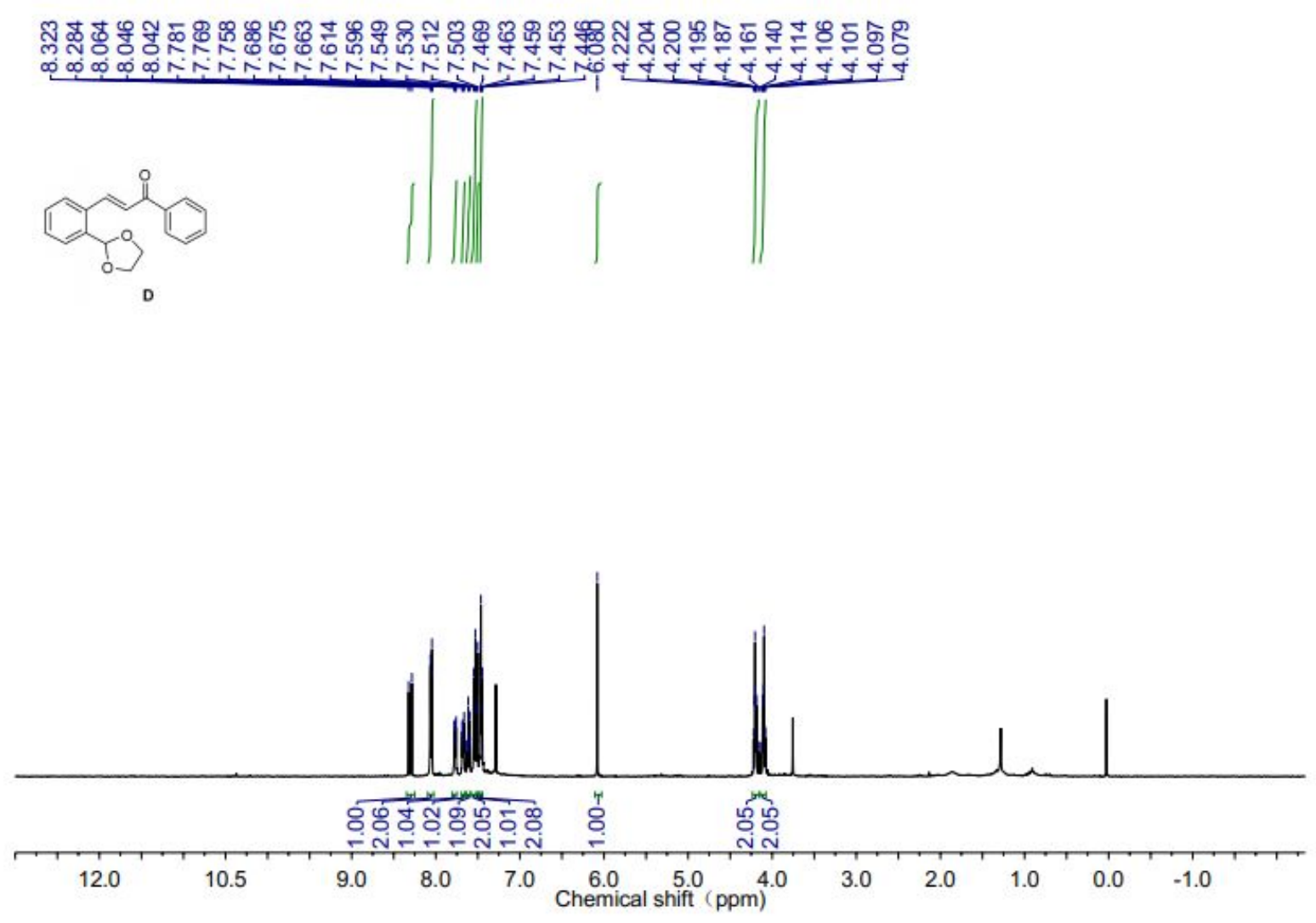

${ }^{1} \mathbf{H}$ NMR $\left(600 \mathrm{MHz}, \mathrm{CDCl}_{3}, 298 \mathrm{~K}\right)$ of $\mathbf{D}$

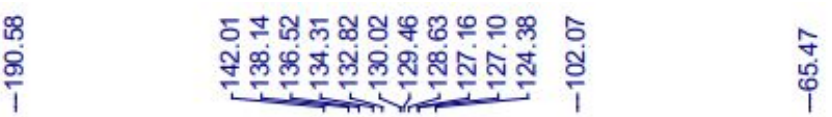
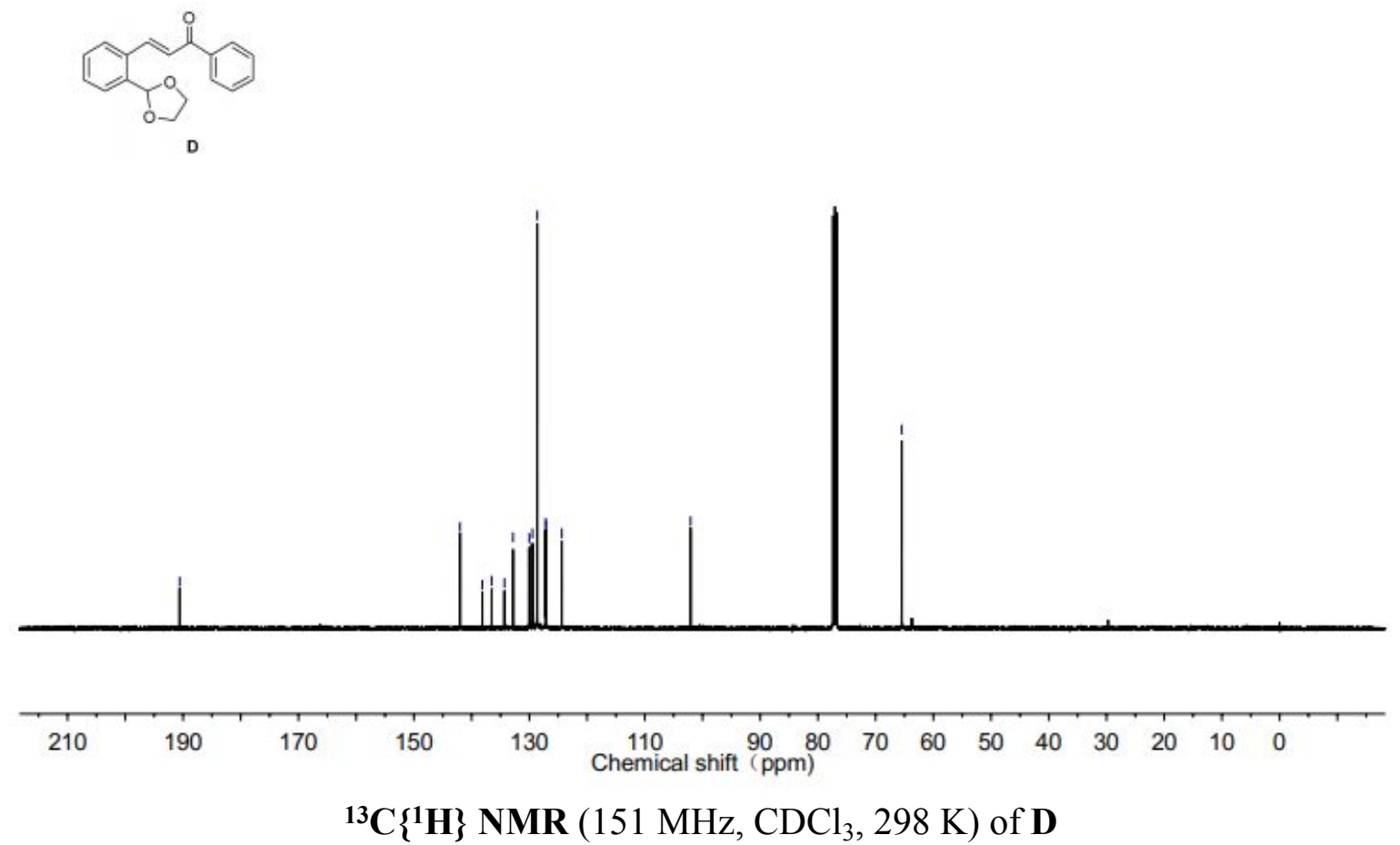

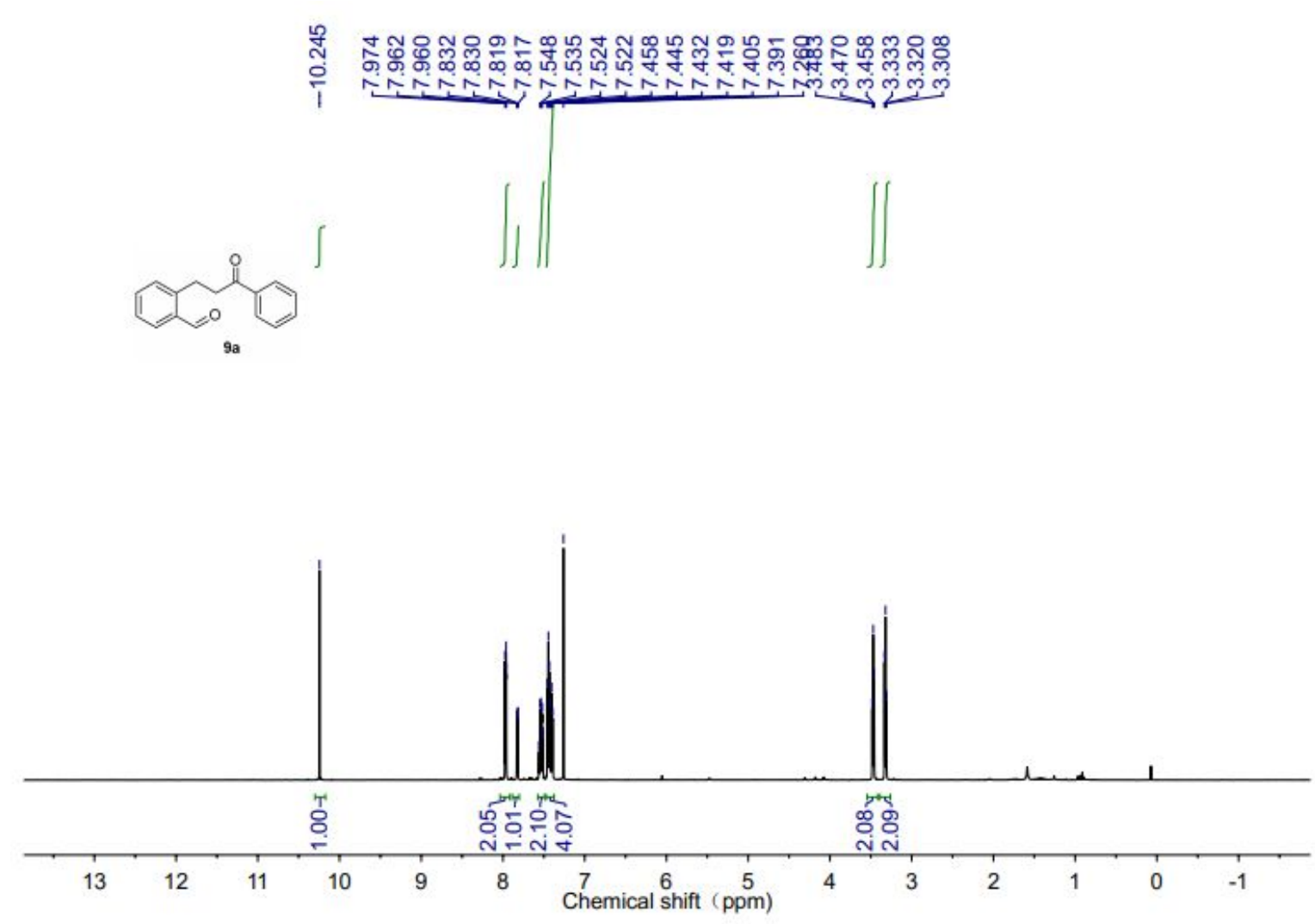

${ }^{1} \mathbf{H}$ NMR $\left(600 \mathrm{MHz}, \mathrm{CDCl}_{3}, 298 \mathrm{~K}\right)$ of 9a

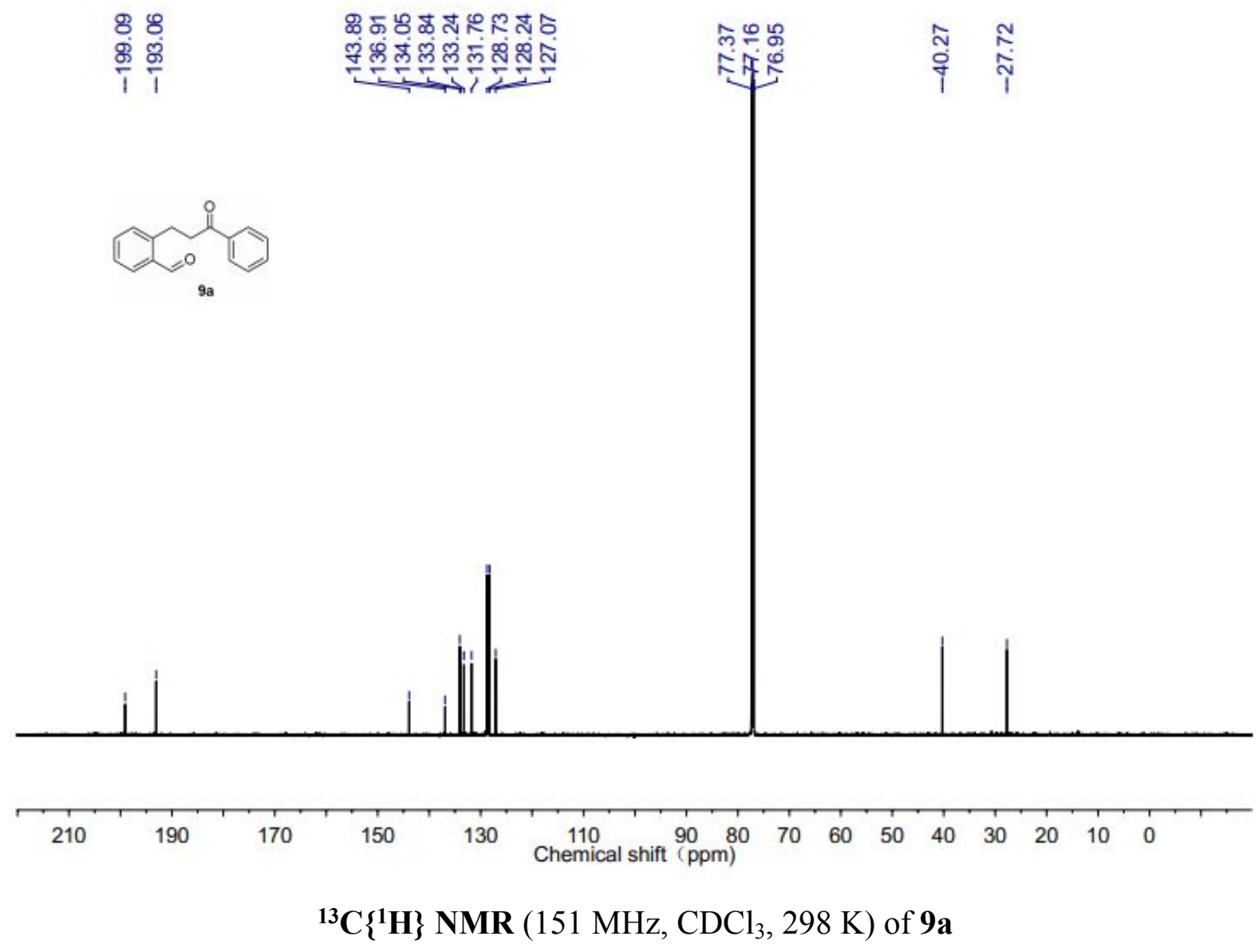



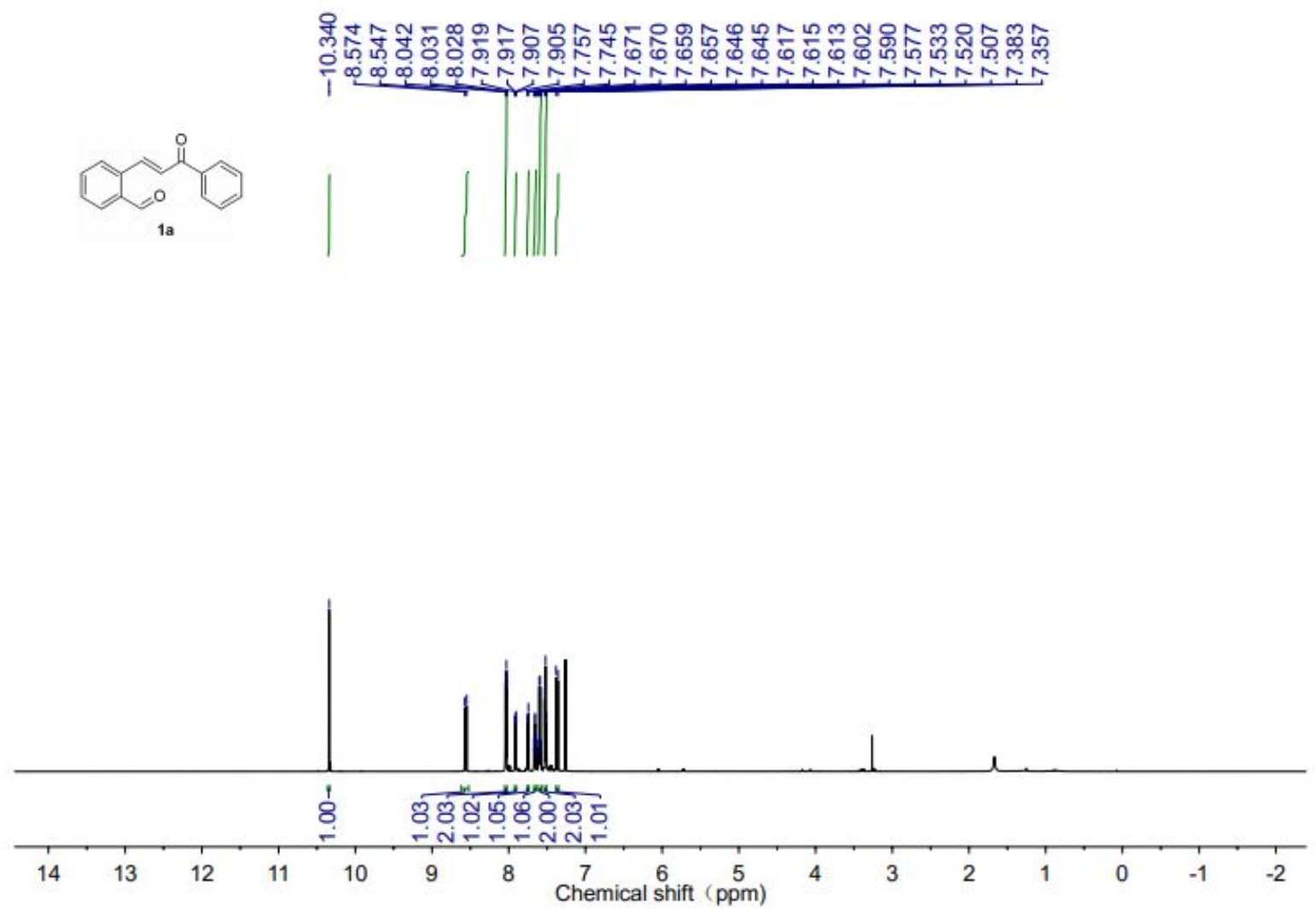

${ }^{1} \mathbf{H}$ NMR $\left(600 \mathrm{MHz}, \mathrm{CDCl}_{3}, 298 \mathrm{~K}\right)$ of $\mathbf{1 a}$

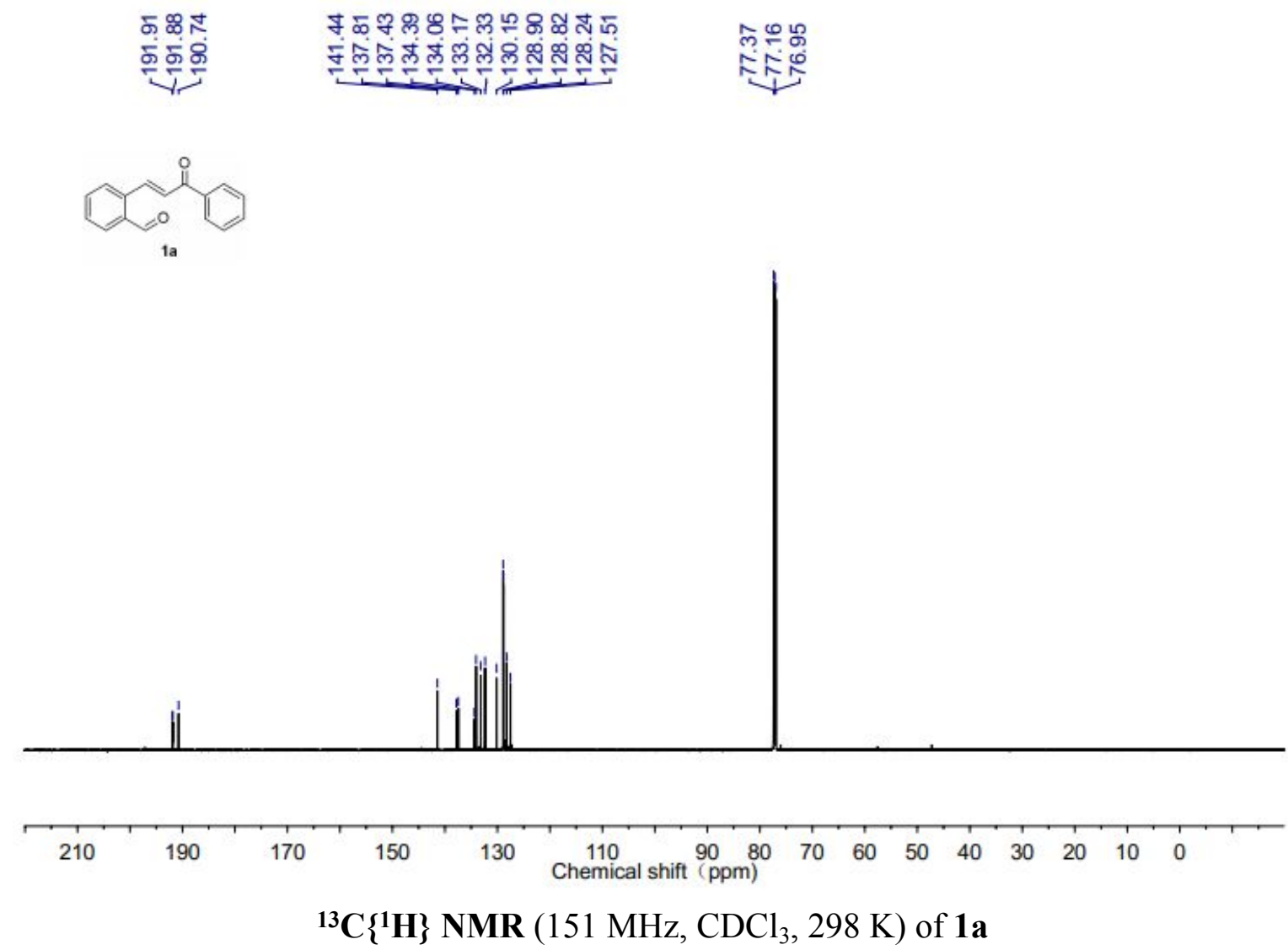



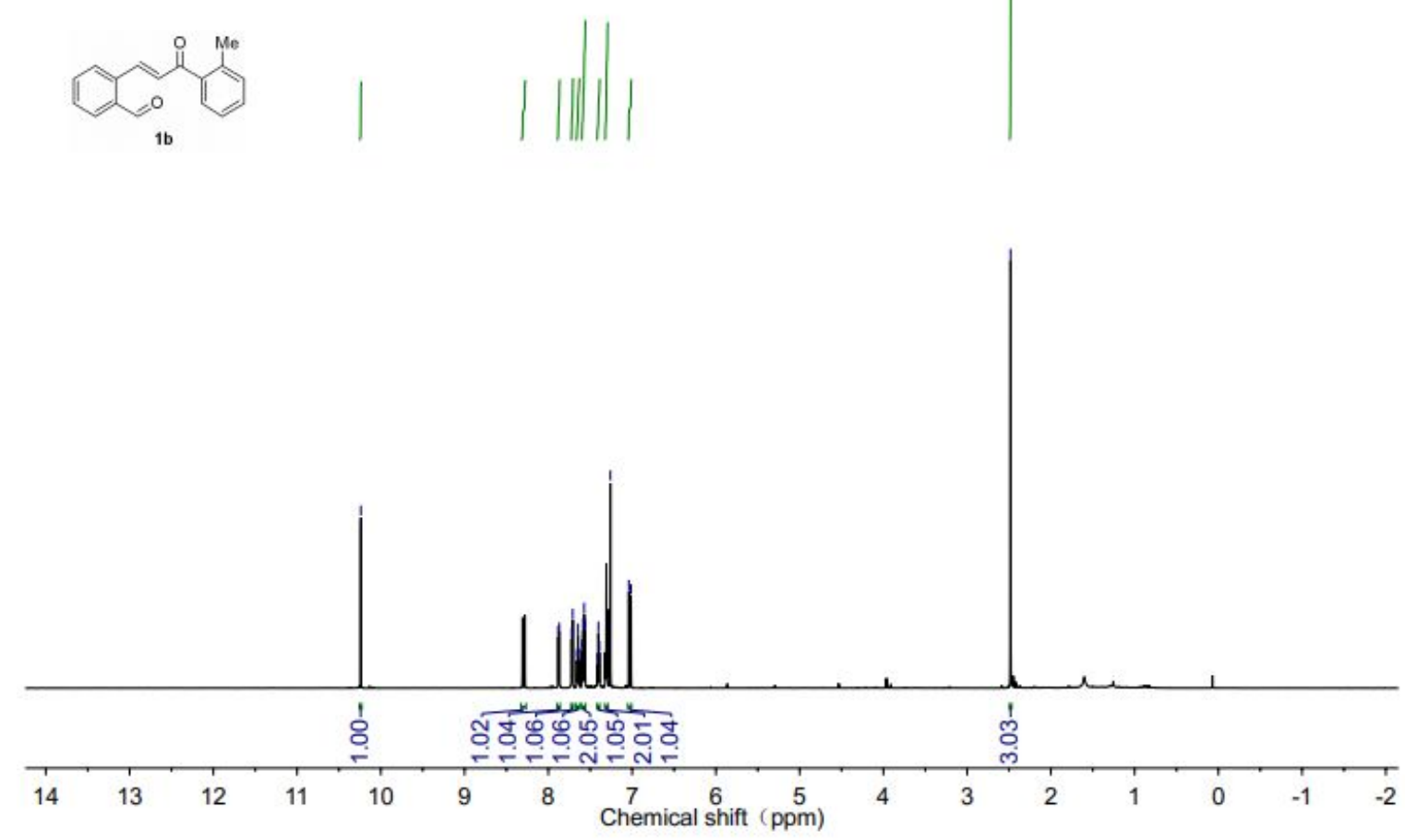

${ }^{1} \mathbf{H}$ NMR $\left(600 \mathrm{MHz}, \mathrm{CDCl}_{3}, 298 \mathrm{~K}\right)$ of $\mathbf{1 b}$
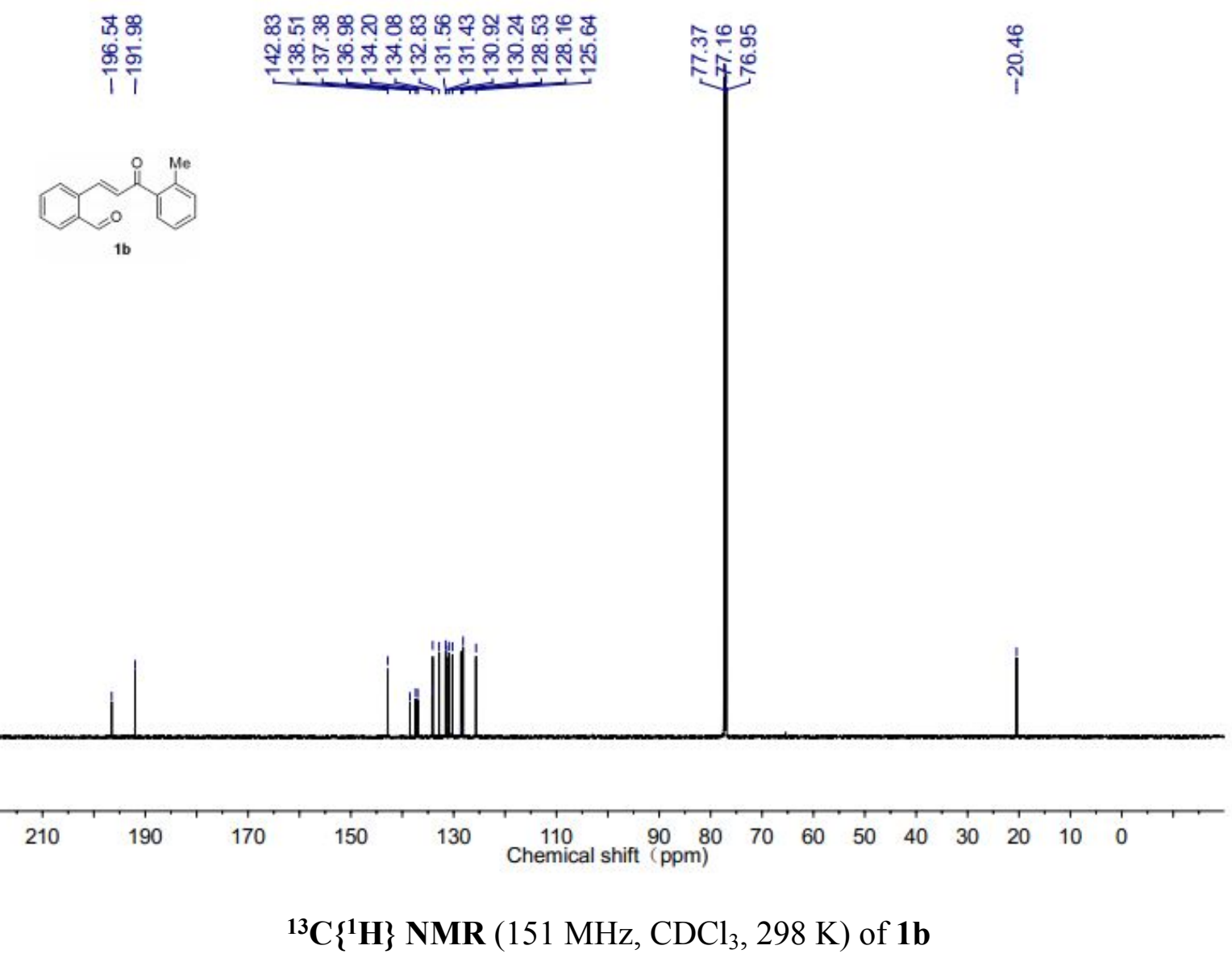

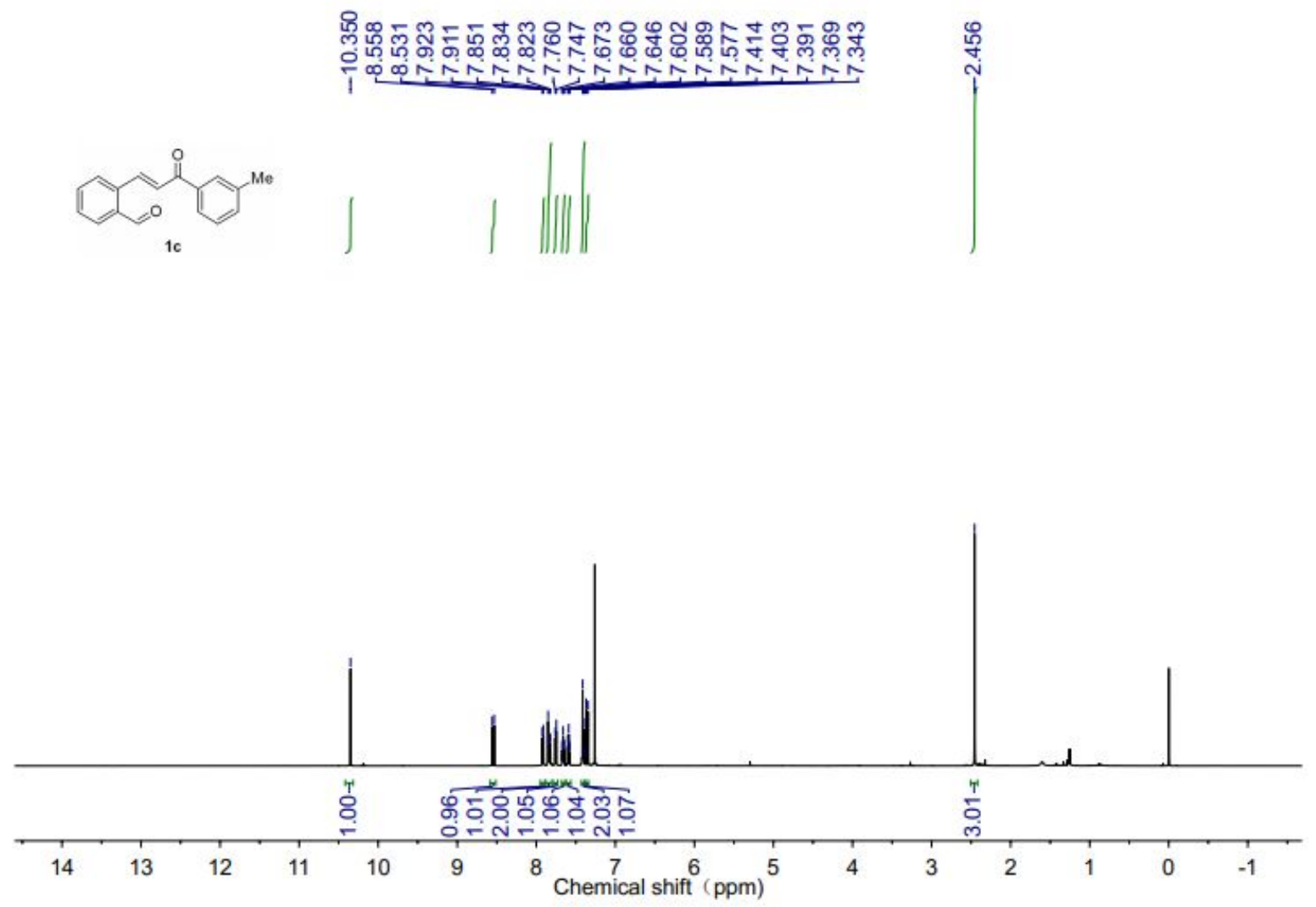

${ }^{1} \mathbf{H}$ NMR $\left(600 \mathrm{MHz}, \mathrm{CDCl}_{3}, 298 \mathrm{~K}\right)$ of $\mathbf{1 c}$

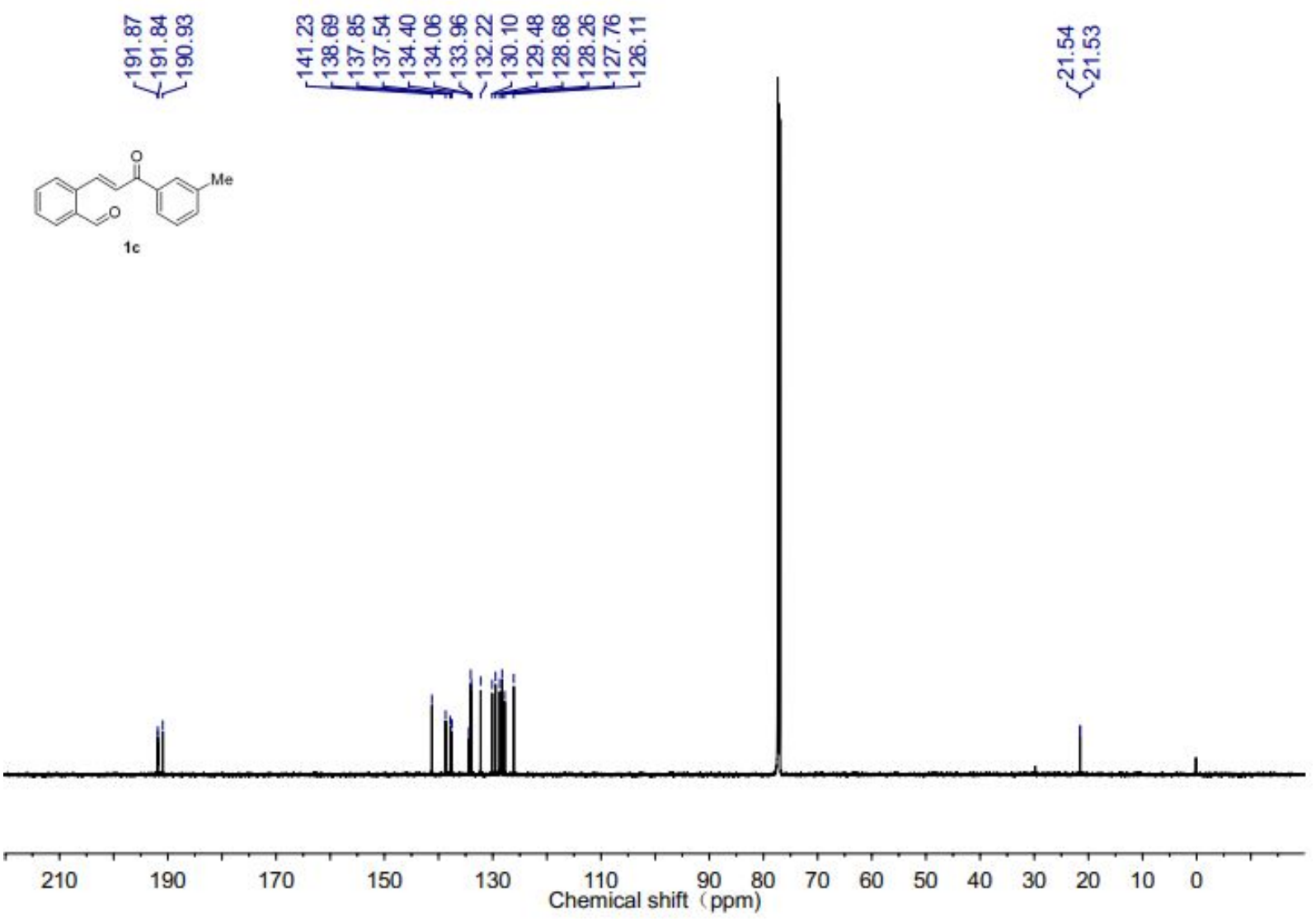


${ }^{13} \mathbf{C}\left\{{ }^{1} \mathbf{H}\right\}$ NMR (151 MHz, $\left.\mathrm{CDCl}_{3}, 298 \mathrm{~K}\right)$ of $\mathbf{1 c}$

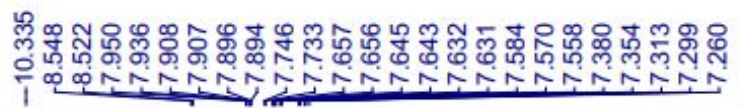

$\underbrace{}_{M e}$
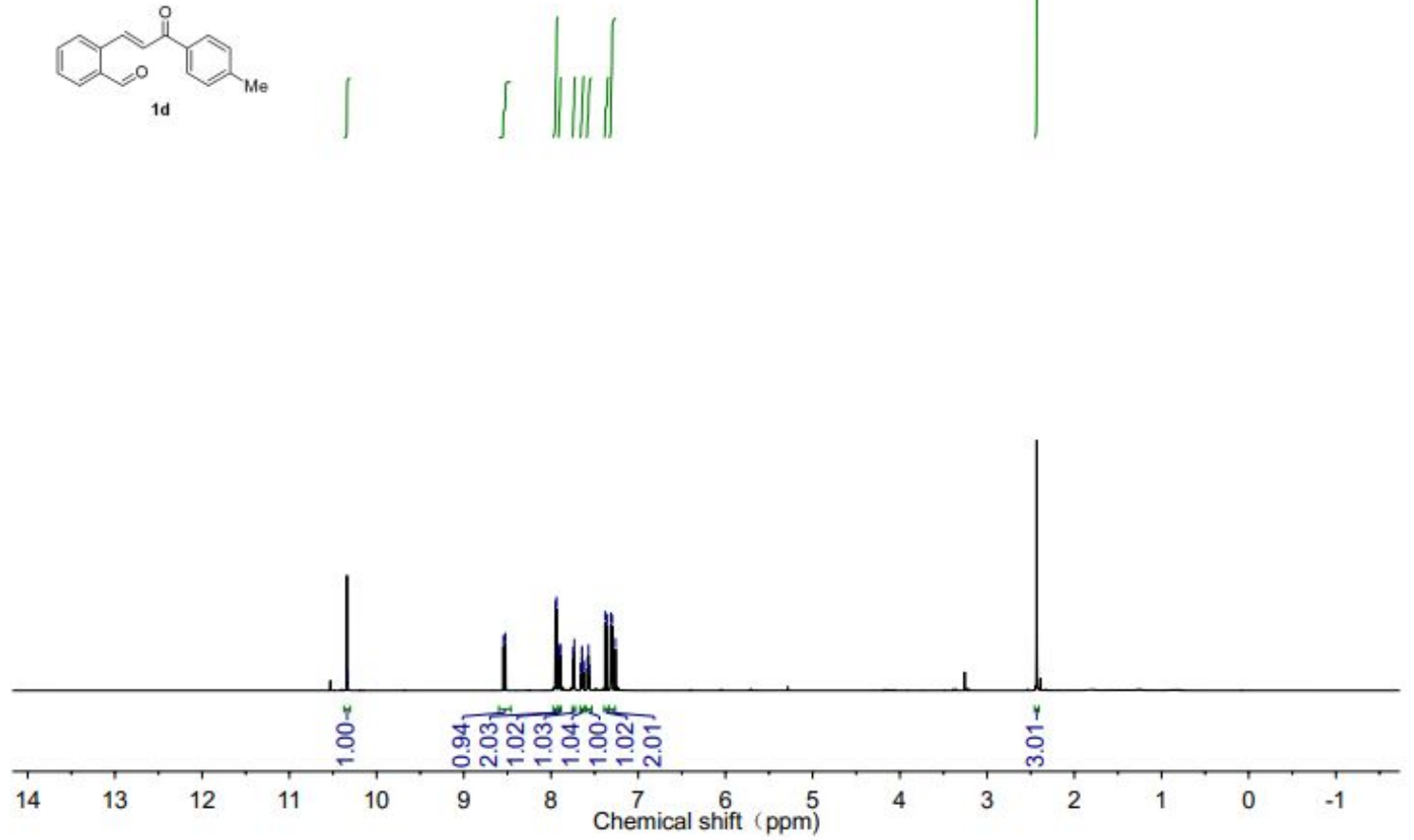

${ }^{1} \mathbf{H}$ NMR $\left(600 \mathrm{MHz}, \mathrm{CDCl}_{3}, 298 \mathrm{~K}\right)$ of $\mathbf{1 d}$

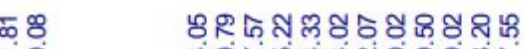

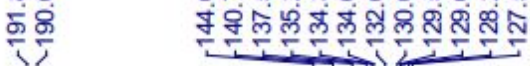

塗是

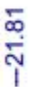
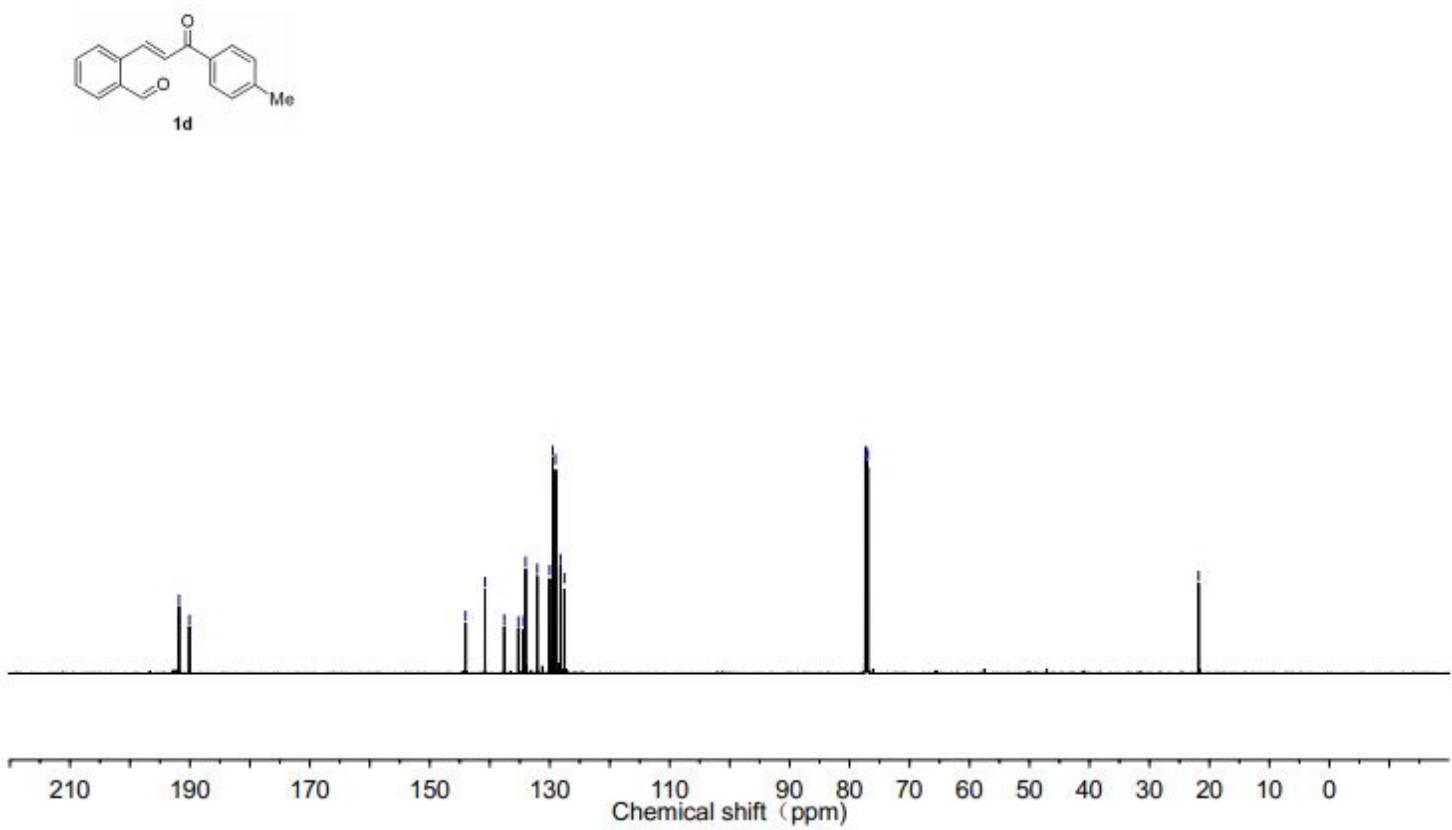
${ }^{13} \mathbf{C}\left\{{ }^{1} \mathbf{H}\right\}$ NMR $\left(151 \mathrm{MHz}, \mathrm{CDCl}_{3}, 298 \mathrm{~K}\right)$ of $\mathbf{1 d}$

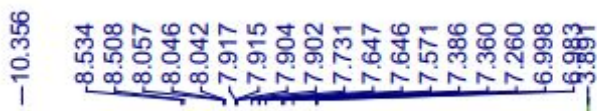
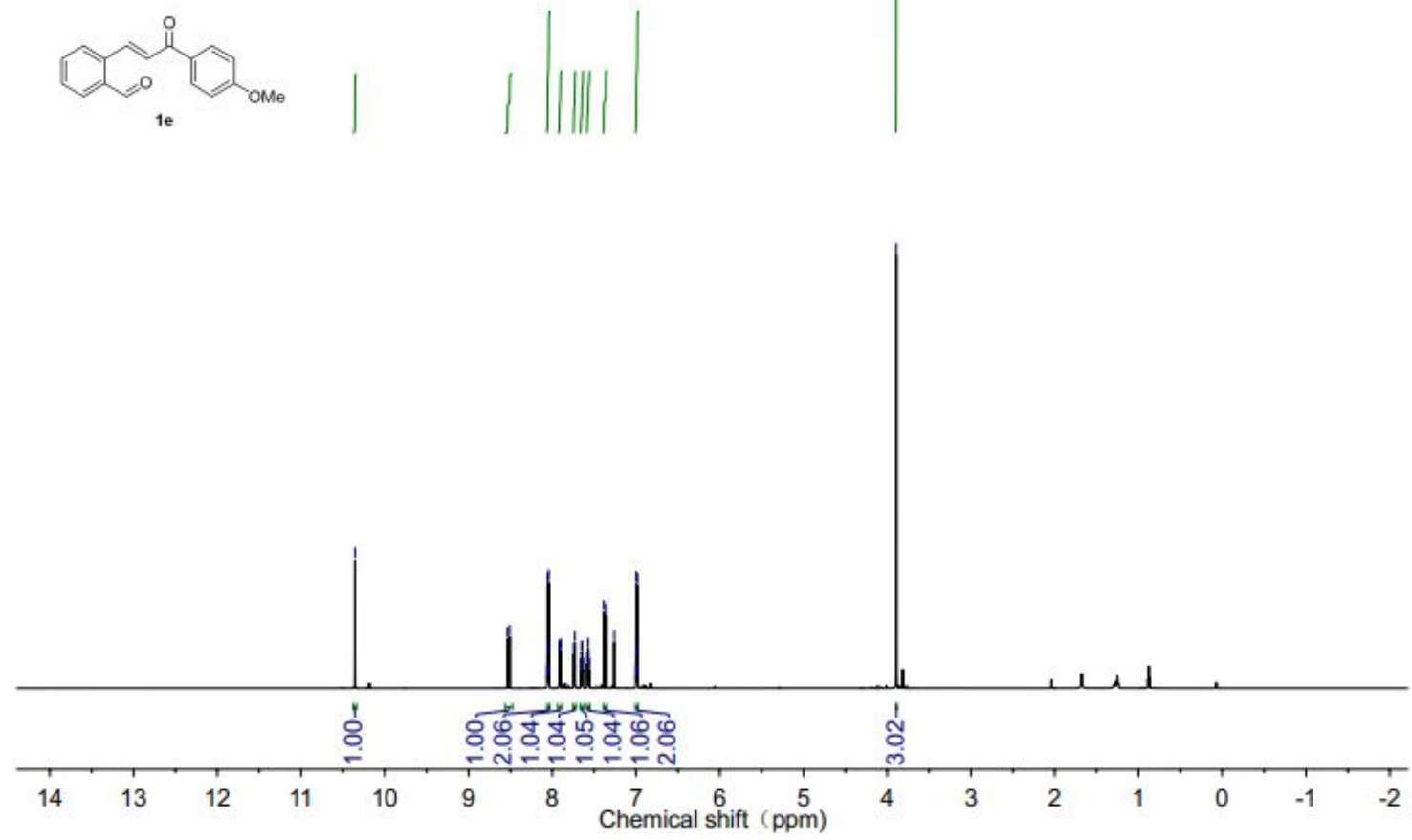

${ }^{1} \mathbf{H}$ NMR $\left(600 \mathrm{MHz}, \mathrm{CDCl}_{3}, 298 \mathrm{~K}\right)$ of $\mathbf{1 e}$
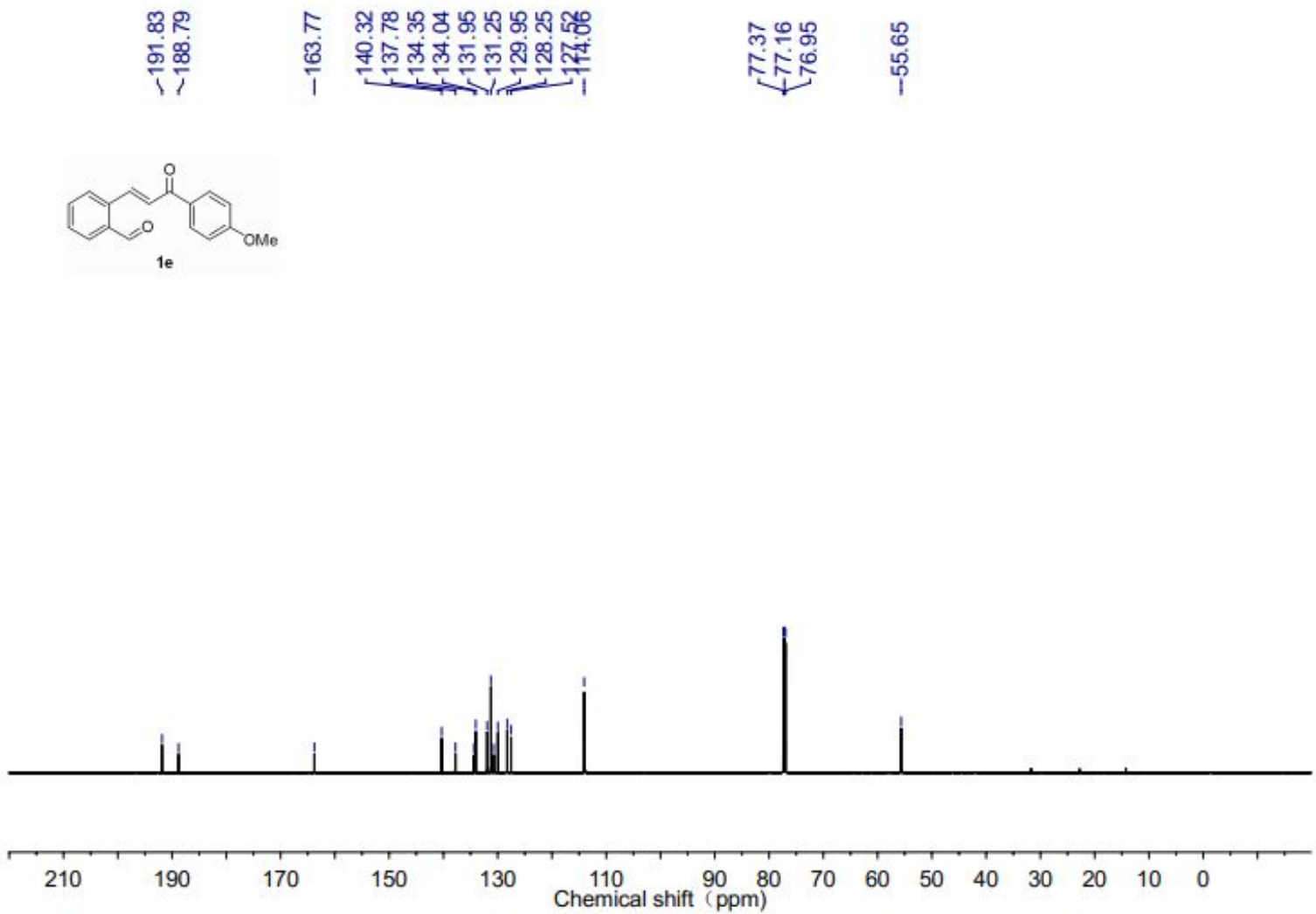
${ }^{13} \mathbf{C}\left\{{ }^{1} \mathbf{H}\right\}$ NMR $\left(151 \mathrm{MHz}, \mathrm{CDCl}_{3}, 298 \mathrm{~K}\right)$ of $\mathbf{1 e}$
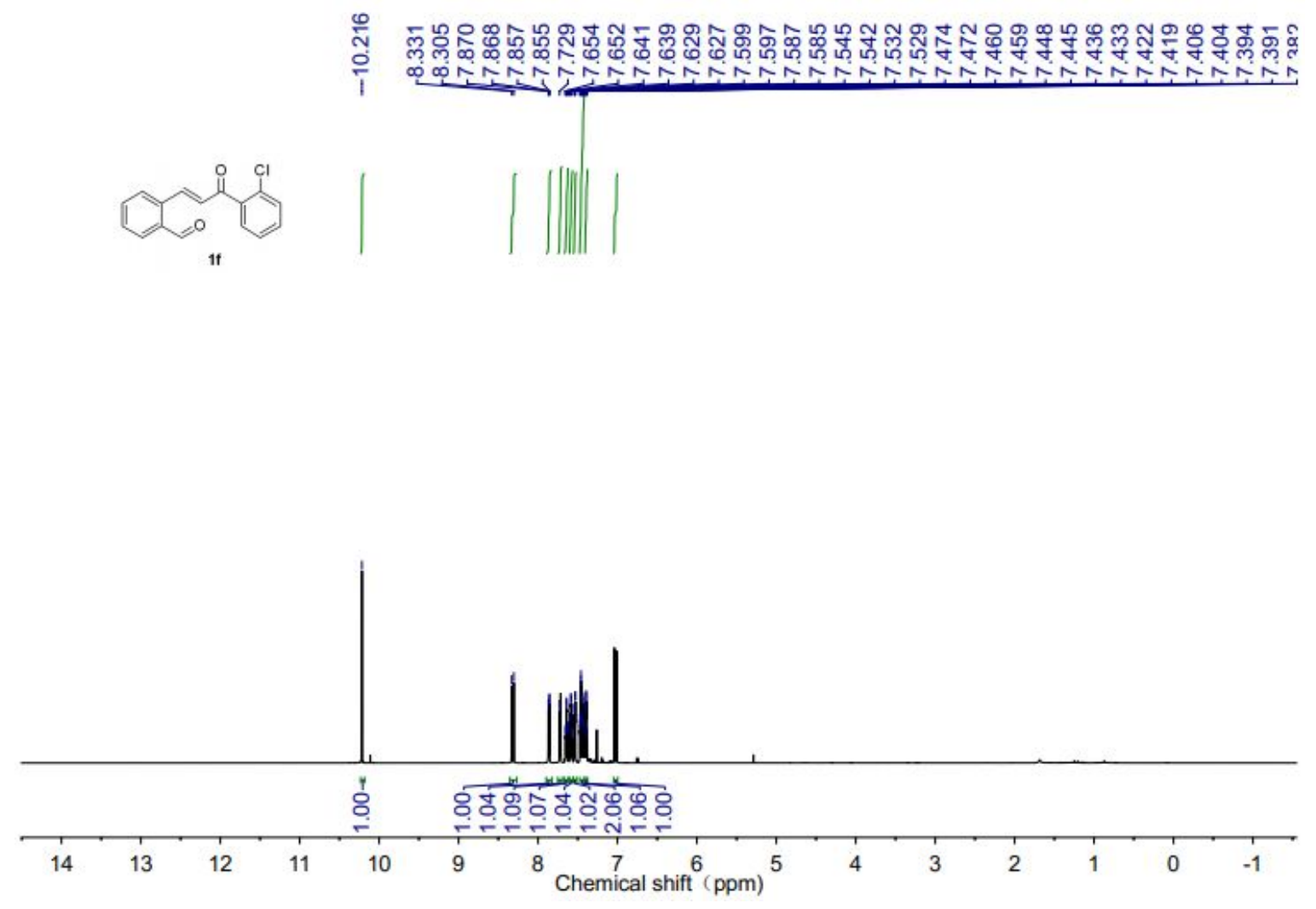

${ }^{\mathbf{1}} \mathbf{H}$ NMR $\left(600 \mathrm{MHz}, \mathrm{CDCl}_{3}, 298 \mathrm{~K}\right)$ of $\mathbf{1 f}$

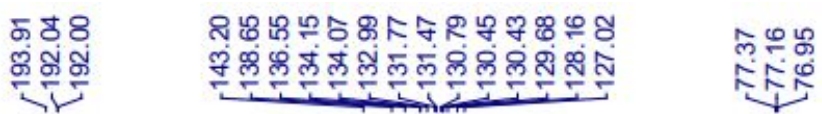
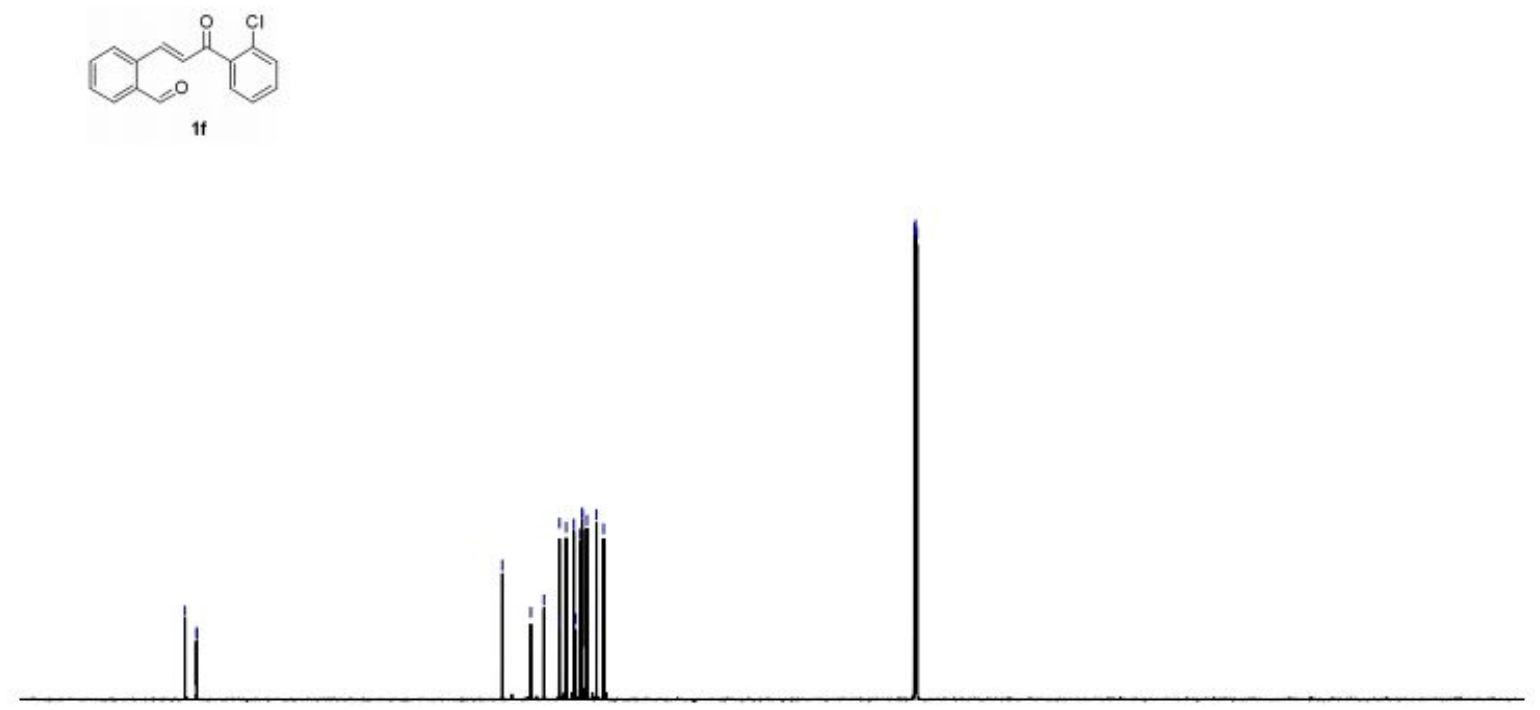

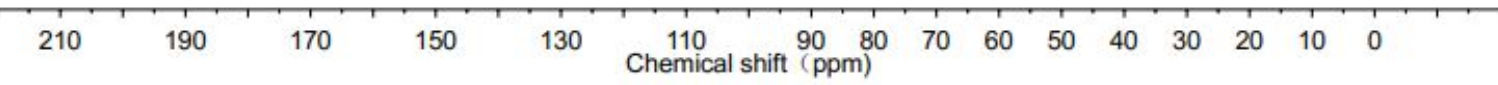




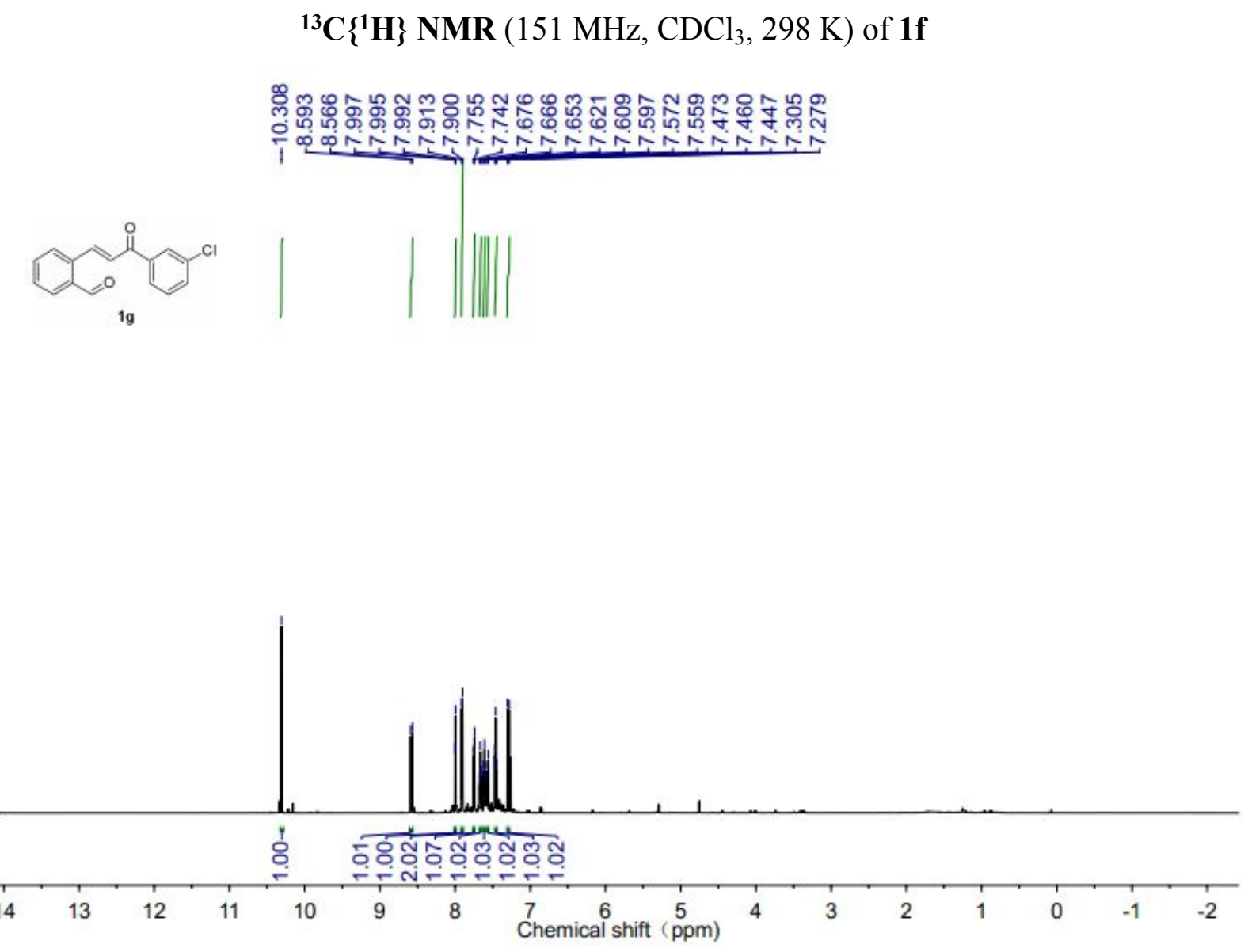

${ }^{\mathbf{1}} \mathbf{H}$ NMR (600 MHz, $\left.\mathrm{CDCl}_{3}, 298 \mathrm{~K}\right)$ of $\mathbf{1 g}$ 

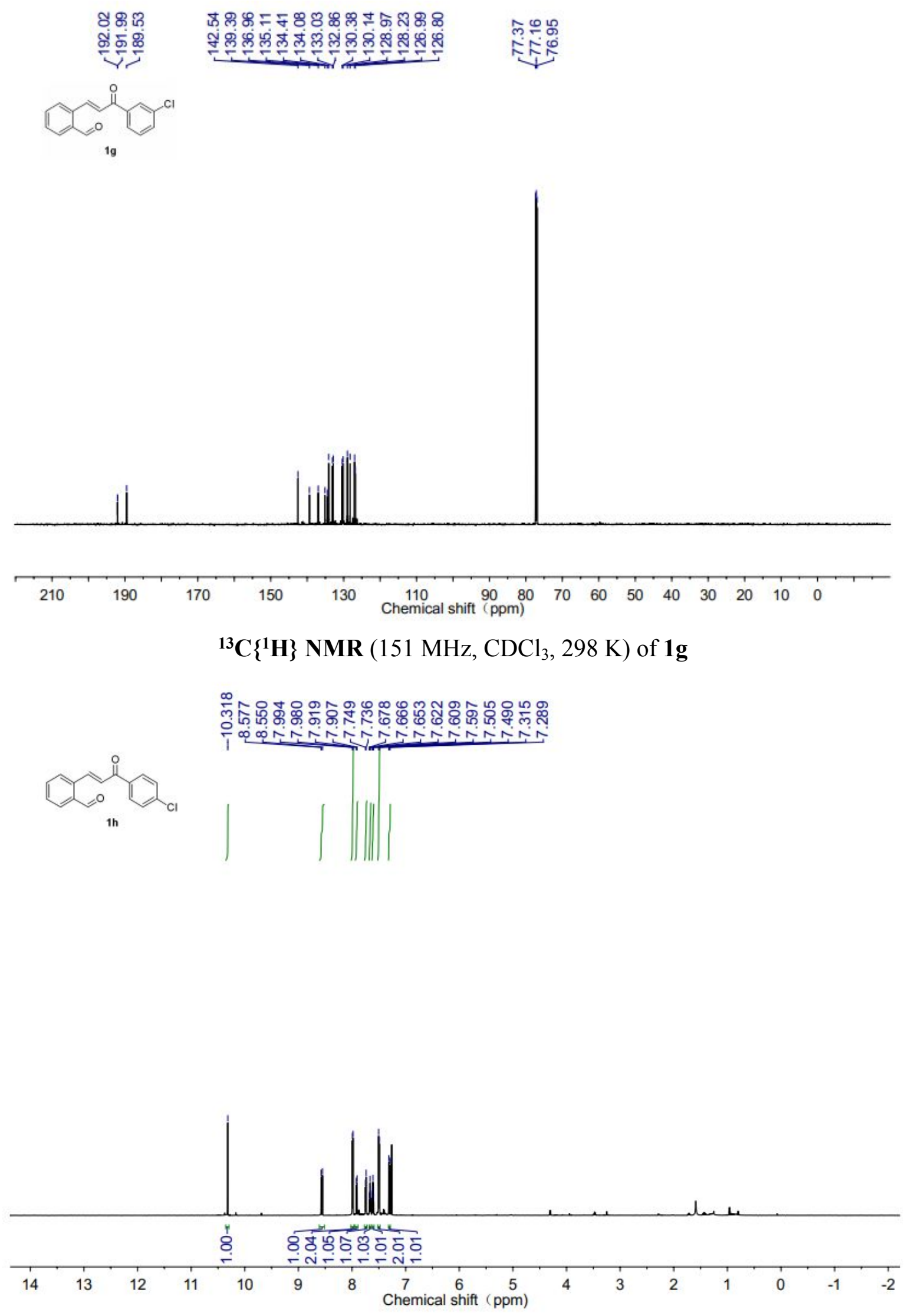

${ }^{\mathbf{1}} \mathbf{H}$ NMR $\left(600 \mathrm{MHz}, \mathrm{CDCl}_{3}, 298 \mathrm{~K}\right)$ of $\mathbf{1 h}$ 


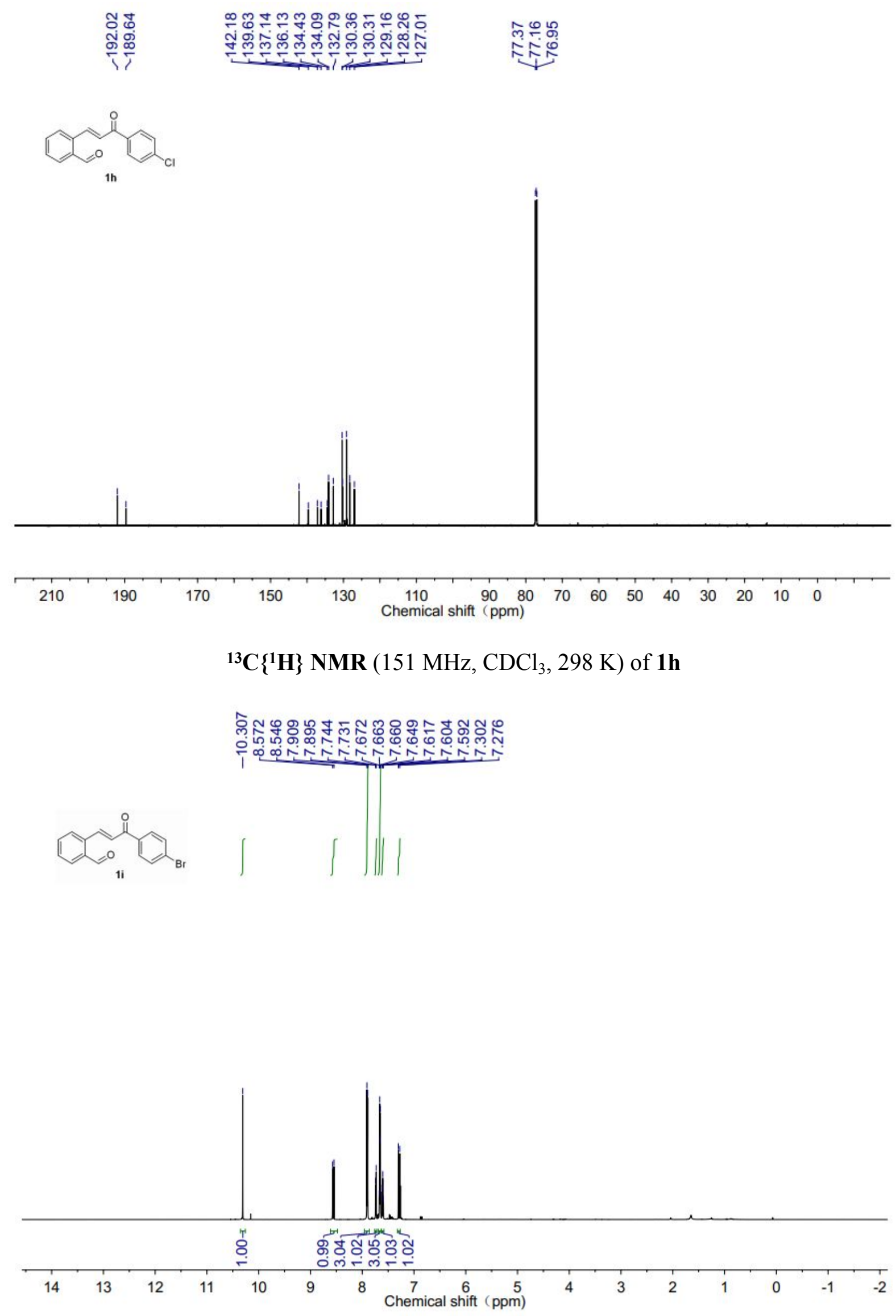


${ }^{1} \mathbf{H}$ NMR $\left(600 \mathrm{MHz}, \mathrm{CDCl}_{3}, 298 \mathrm{~K}\right)$ of $\mathbf{1 i}$

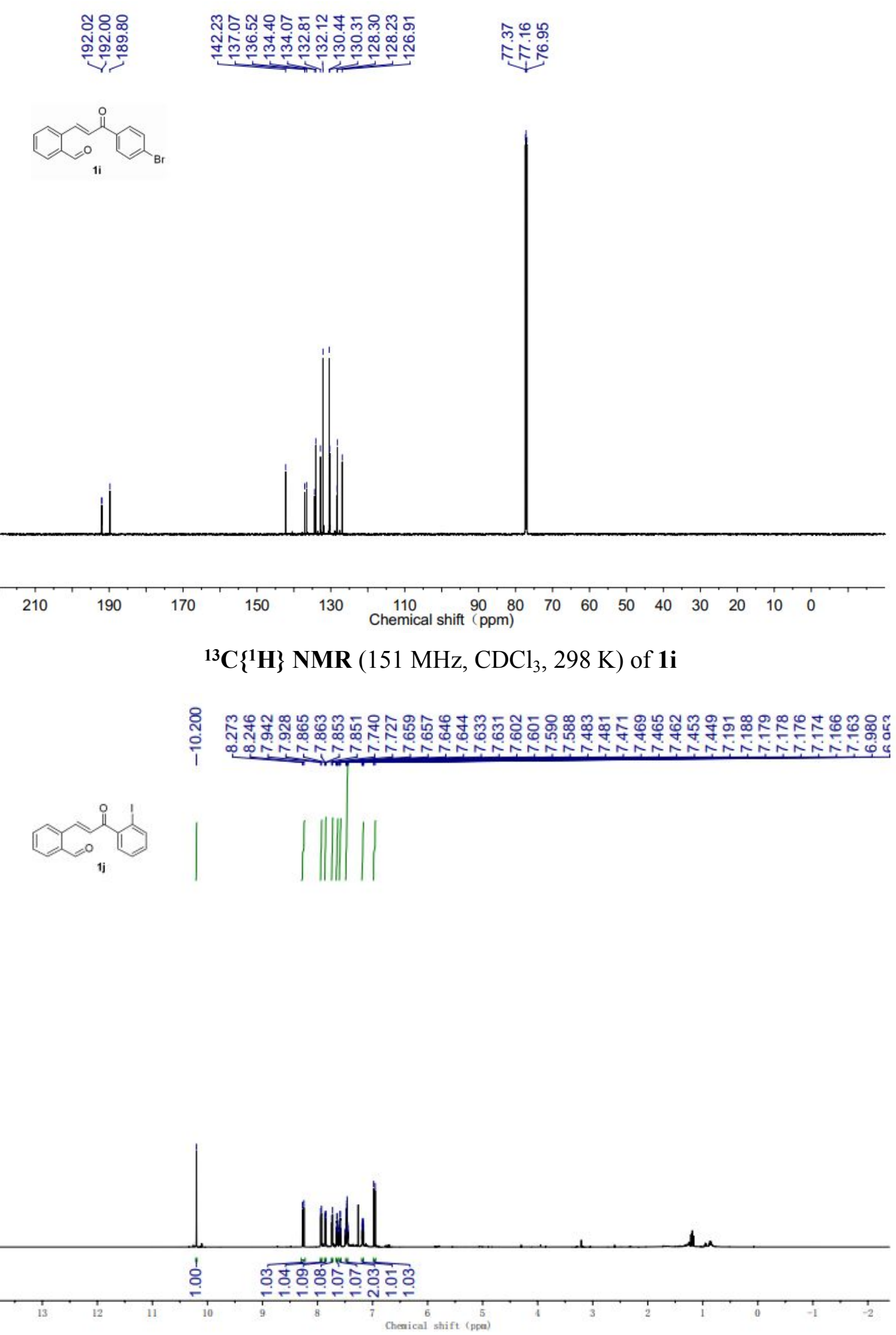


${ }^{1} \mathbf{H}$ NMR $\left(600 \mathrm{MHz}, \mathrm{CDCl}_{3}, 298 \mathrm{~K}\right)$ of $\mathbf{1 j}$
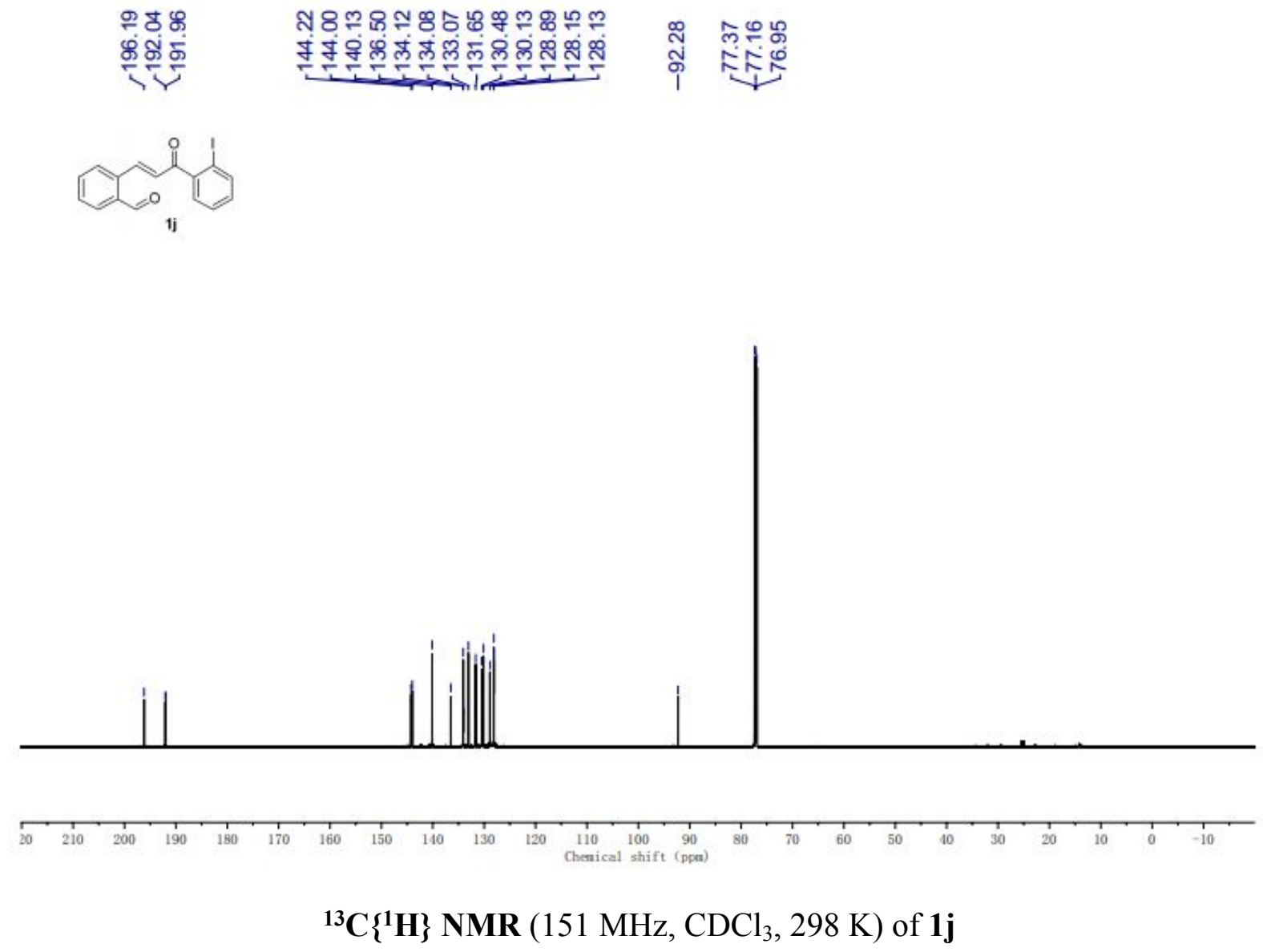

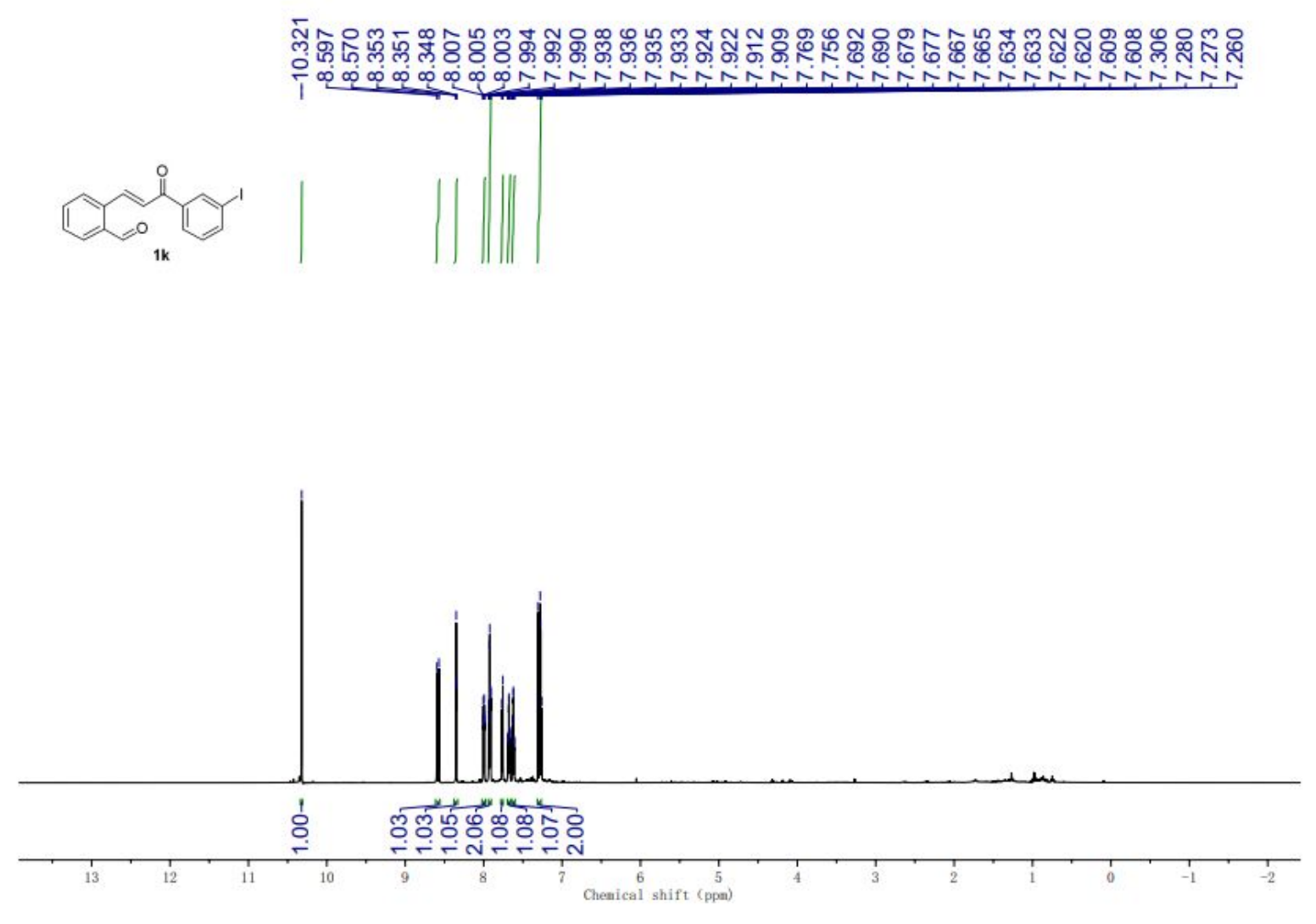

${ }^{\mathbf{1}} \mathbf{H}$ NMR $\left(600 \mathrm{MHz}, \mathrm{CDCl}_{3}, 298 \mathrm{~K}\right)$ of $\mathbf{1 k}$

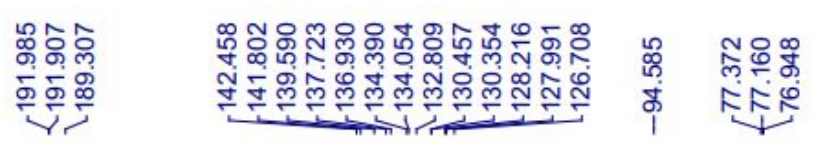
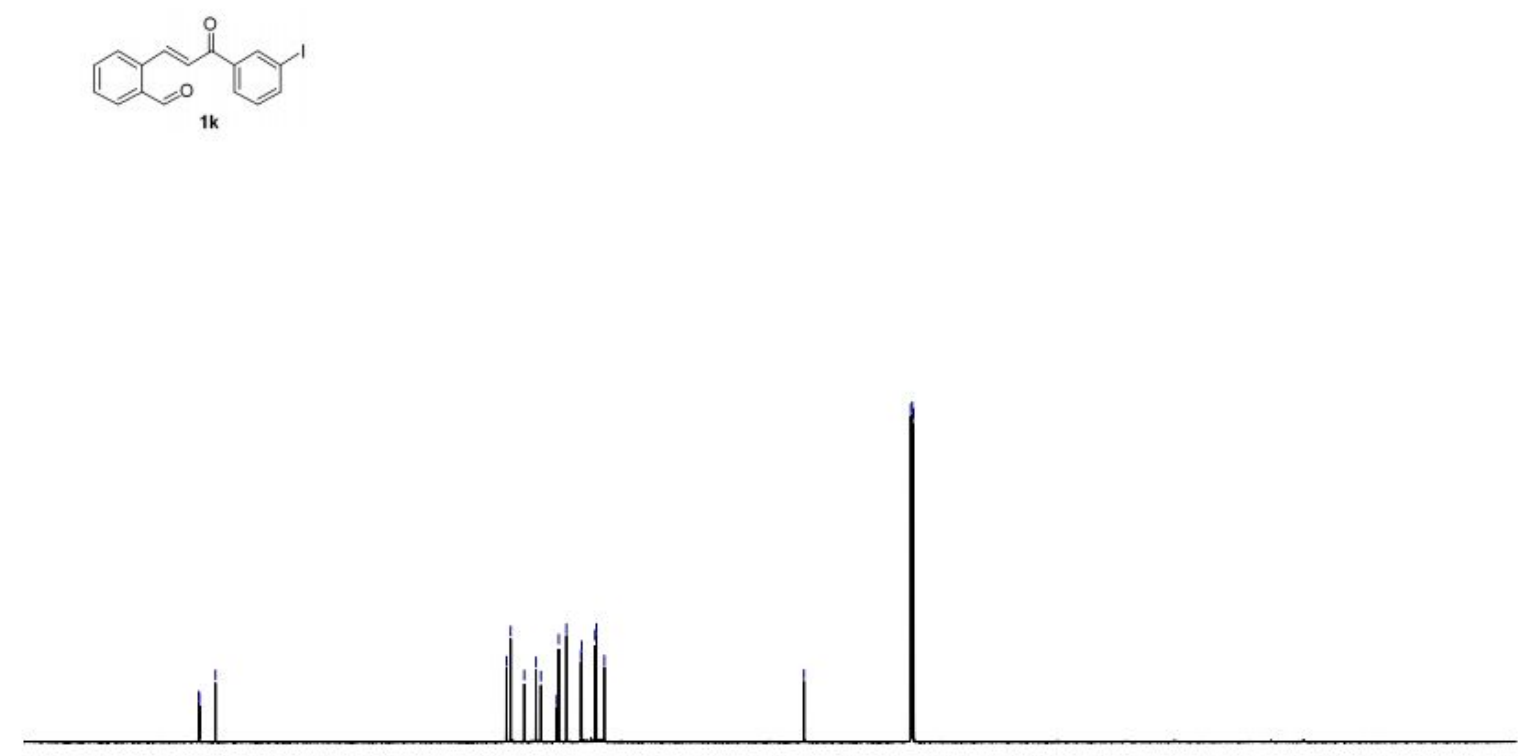

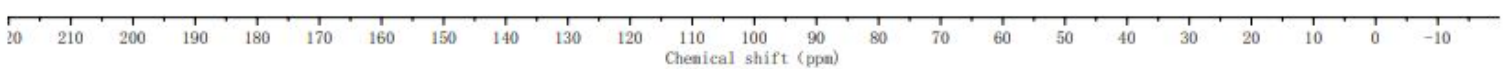


${ }^{13} \mathbf{C}\left\{{ }^{1} \mathbf{H}\right\}$ NMR $\left(151 \mathrm{MHz}, \mathrm{CDCl}_{3}, 298 \mathrm{~K}\right)$ of $\mathbf{1 k}$
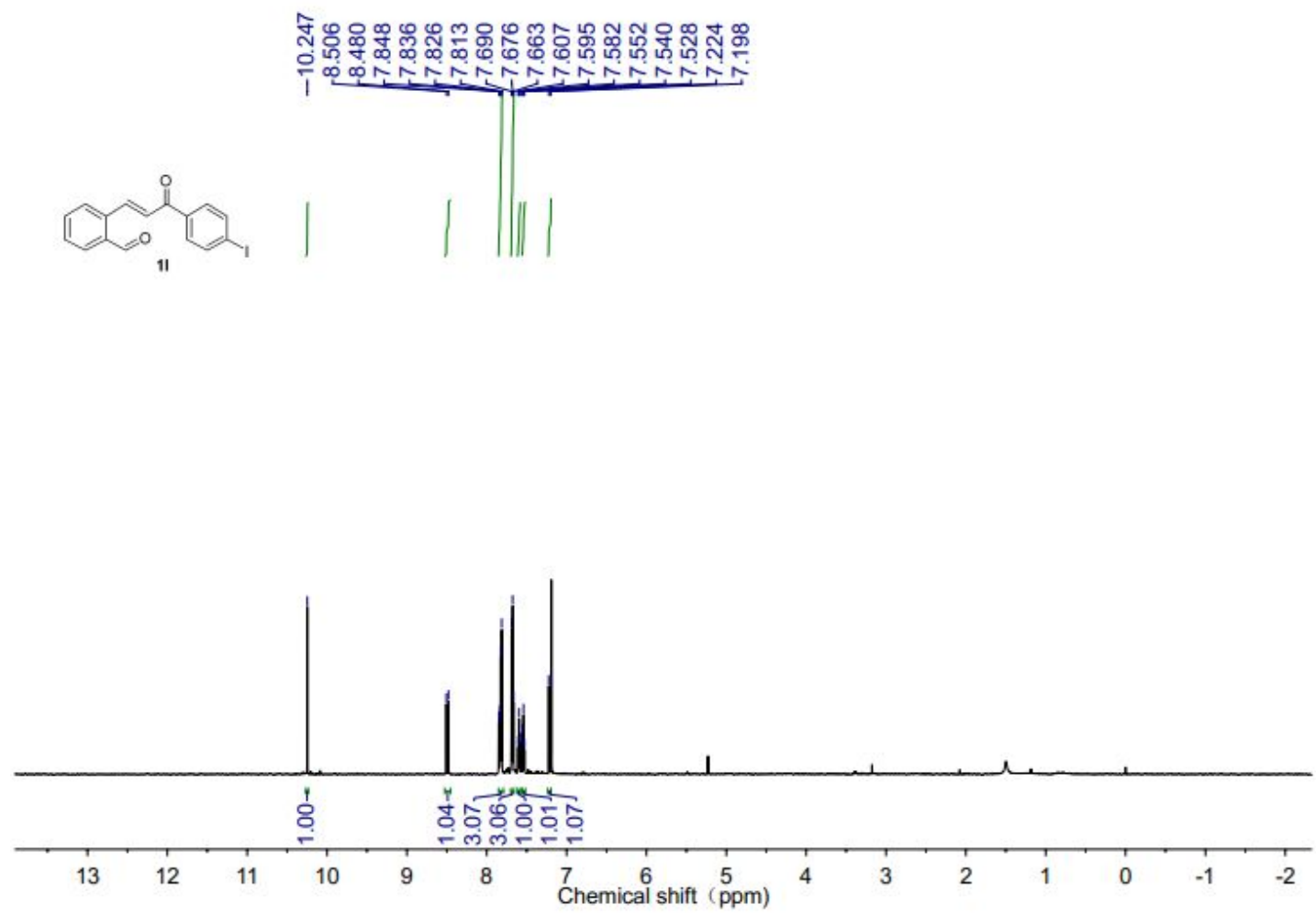

${ }^{\mathbf{1}} \mathbf{H}$ NMR $\left(600 \mathrm{MHz}, \mathrm{CDCl}_{3}, 298 \mathrm{~K}\right)$ of $\mathbf{1 I}$
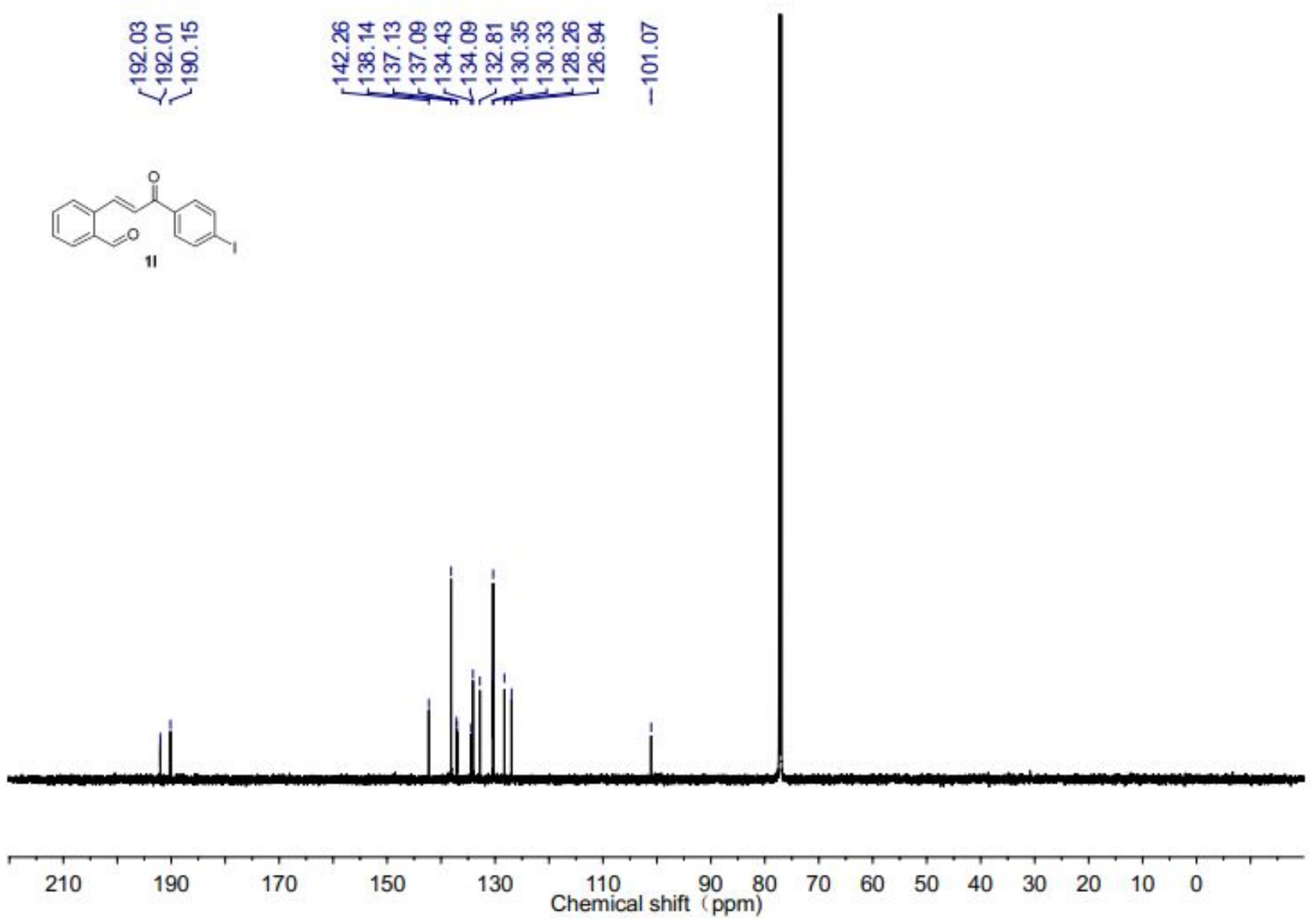
${ }^{13} \mathbf{C}\left\{{ }^{1} \mathbf{H}\right\}$ NMR $\left(151 \mathrm{MHz}, \mathrm{CDCl}_{3}, 298 \mathrm{~K}\right)$ of $\mathbf{1 l}$

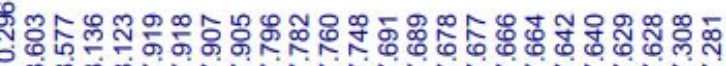

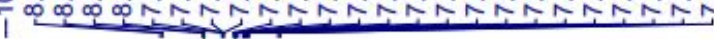
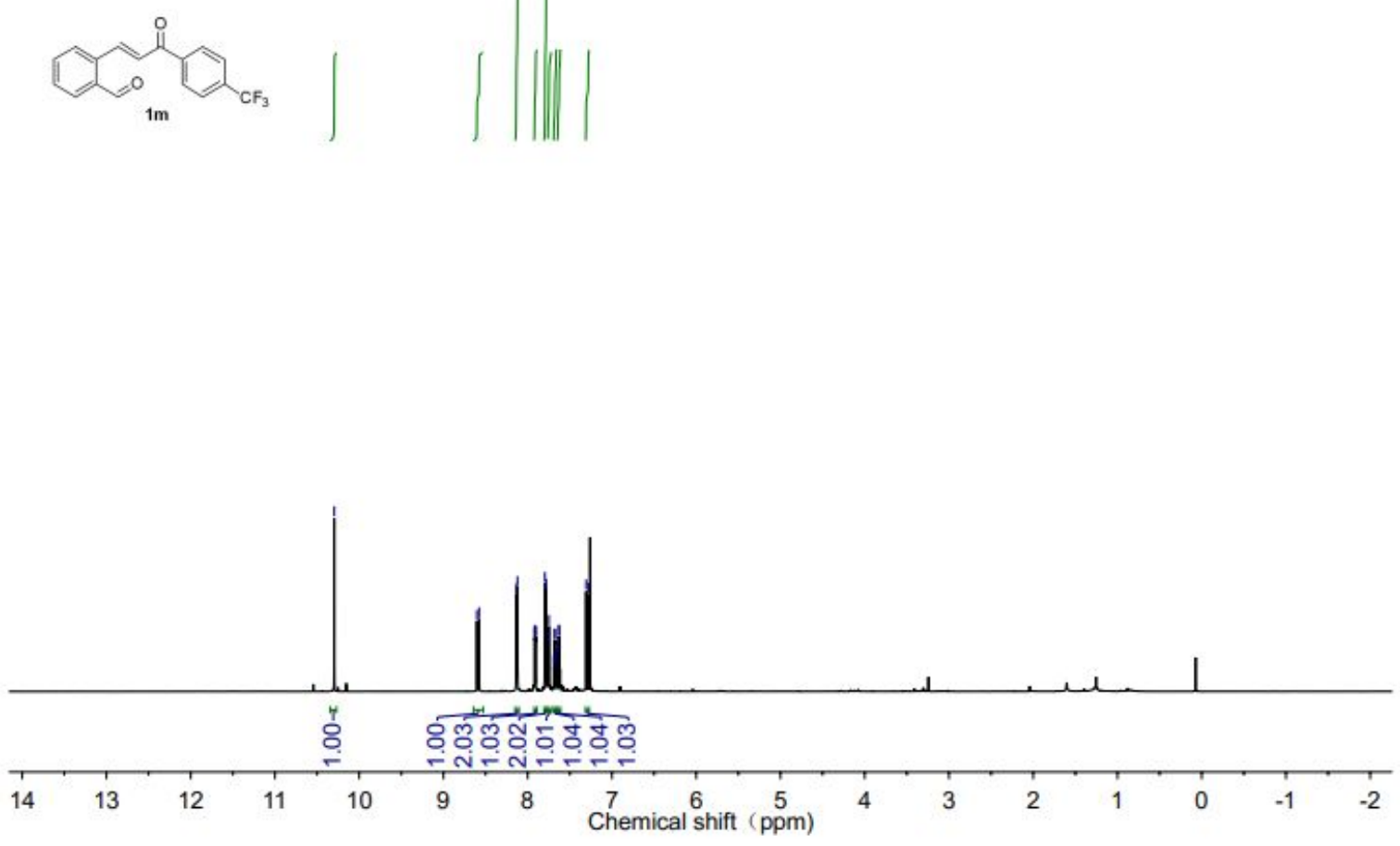

${ }^{1} \mathbf{H}$ NMR $\left(600 \mathrm{MHz}, \mathrm{CDCl}_{3}, 298 \mathrm{~K}\right)$ of $\mathbf{1} \mathbf{m}$

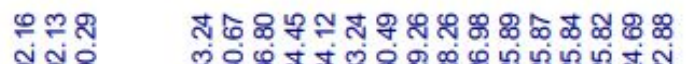

สู่ร

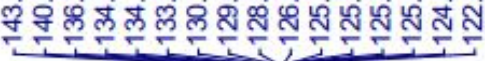

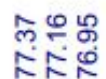
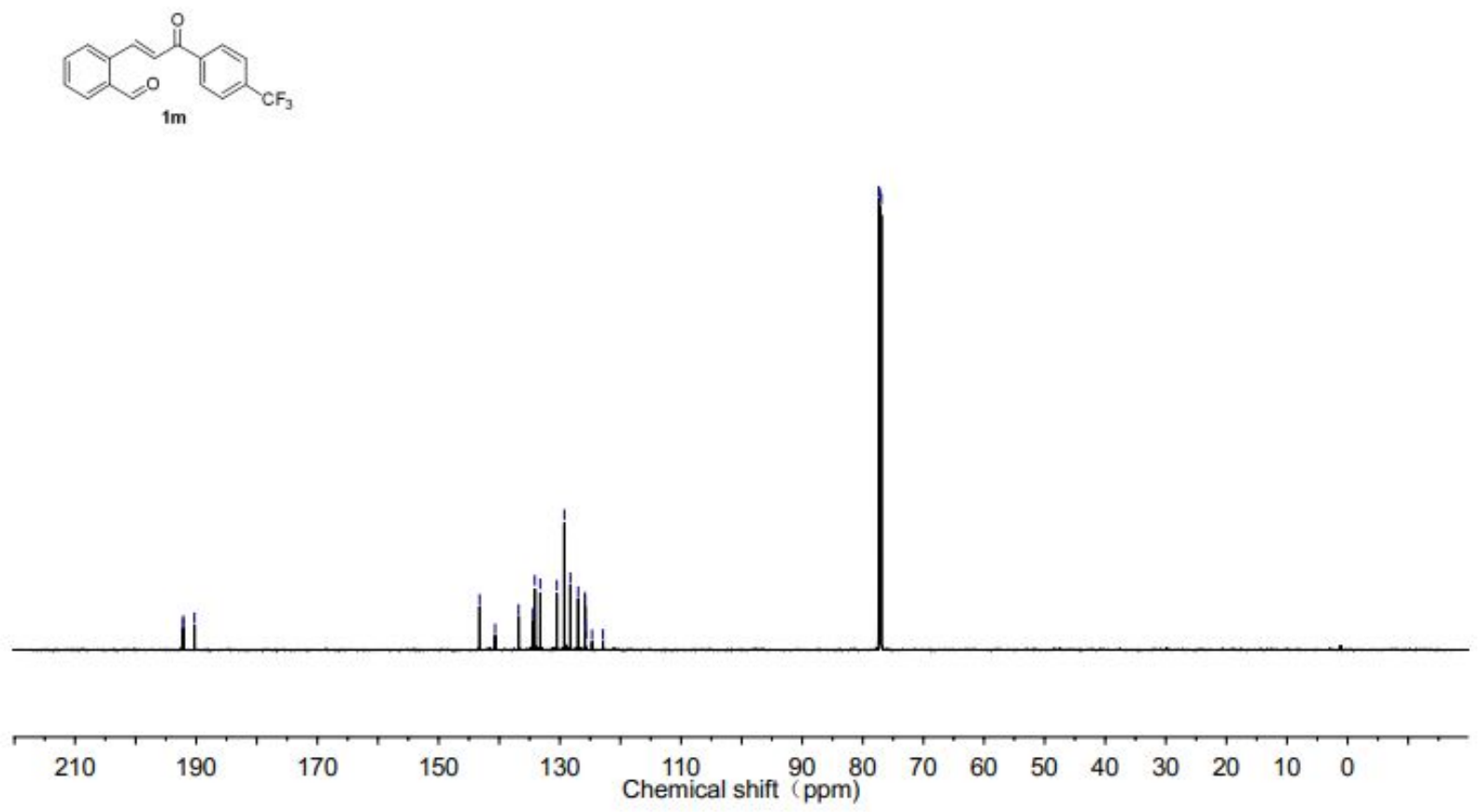
${ }^{13} \mathbf{C}\left\{{ }^{1} \mathbf{H}\right\}$ NMR $\left(151 \mathrm{MHz}, \mathrm{CDCl}_{3}, 298 \mathrm{~K}\right)$ of $\mathbf{1 m}$

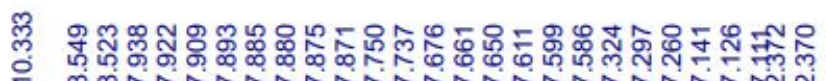

i
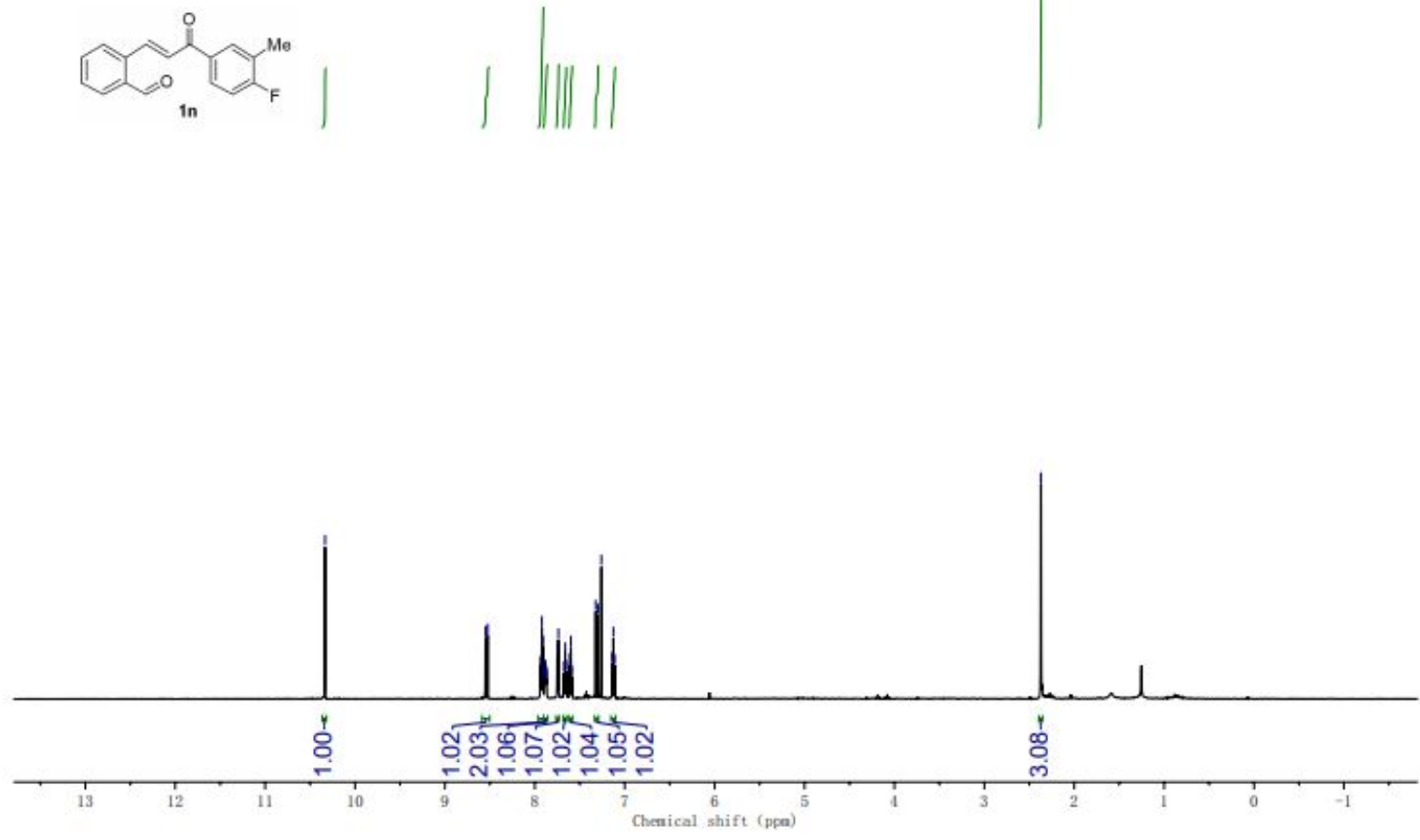

${ }^{\mathbf{1}} \mathbf{H}$ NMR $\left(600 \mathrm{MHz}, \mathrm{CDCl}_{3}, 298 \mathrm{~K}\right)$ of $\mathbf{1 n}$ 

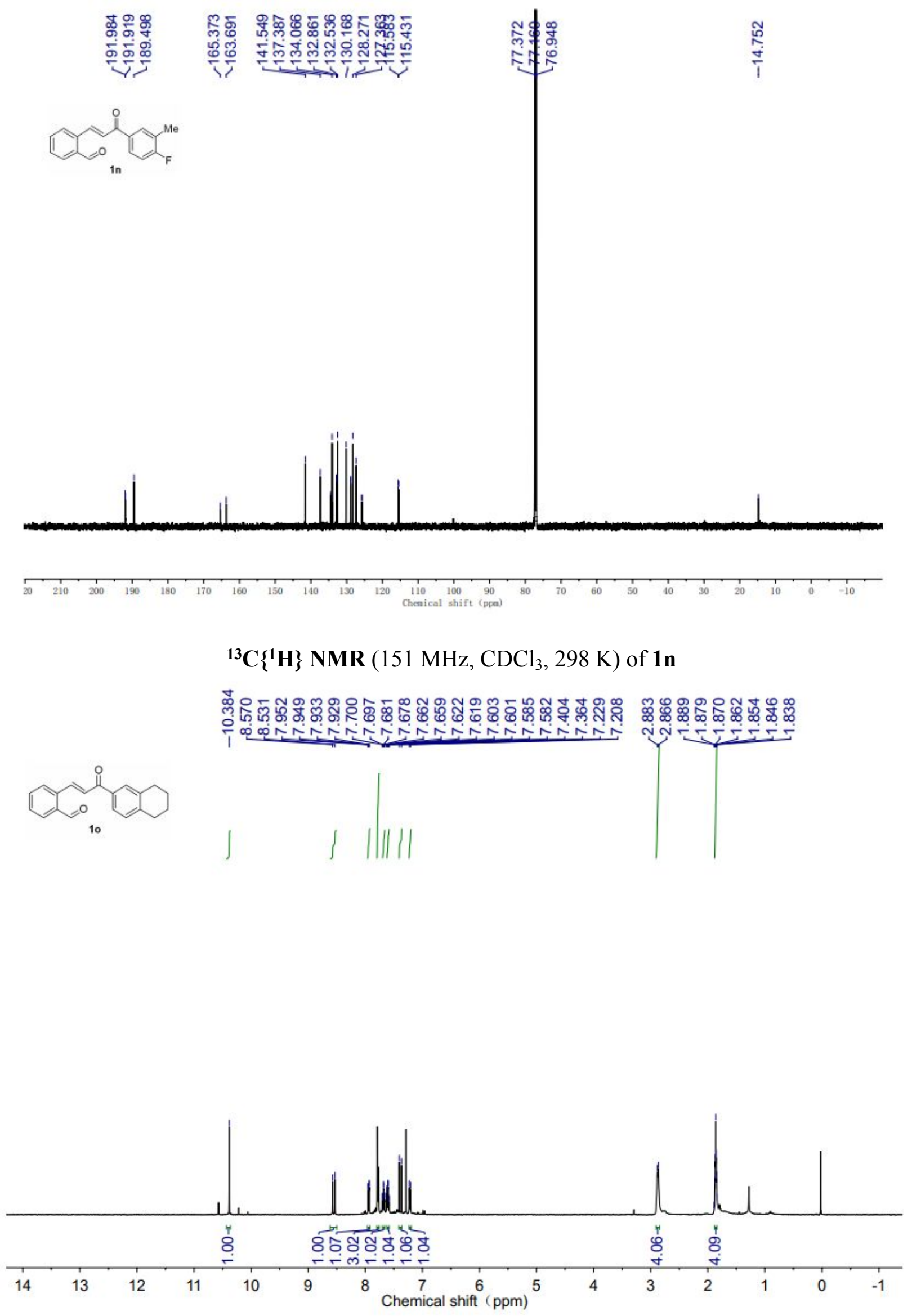
${ }^{1} \mathbf{H}$ NMR $\left(600 \mathrm{MHz}, \mathrm{CDCl}_{3}, 298 \mathrm{~K}\right)$ of 10

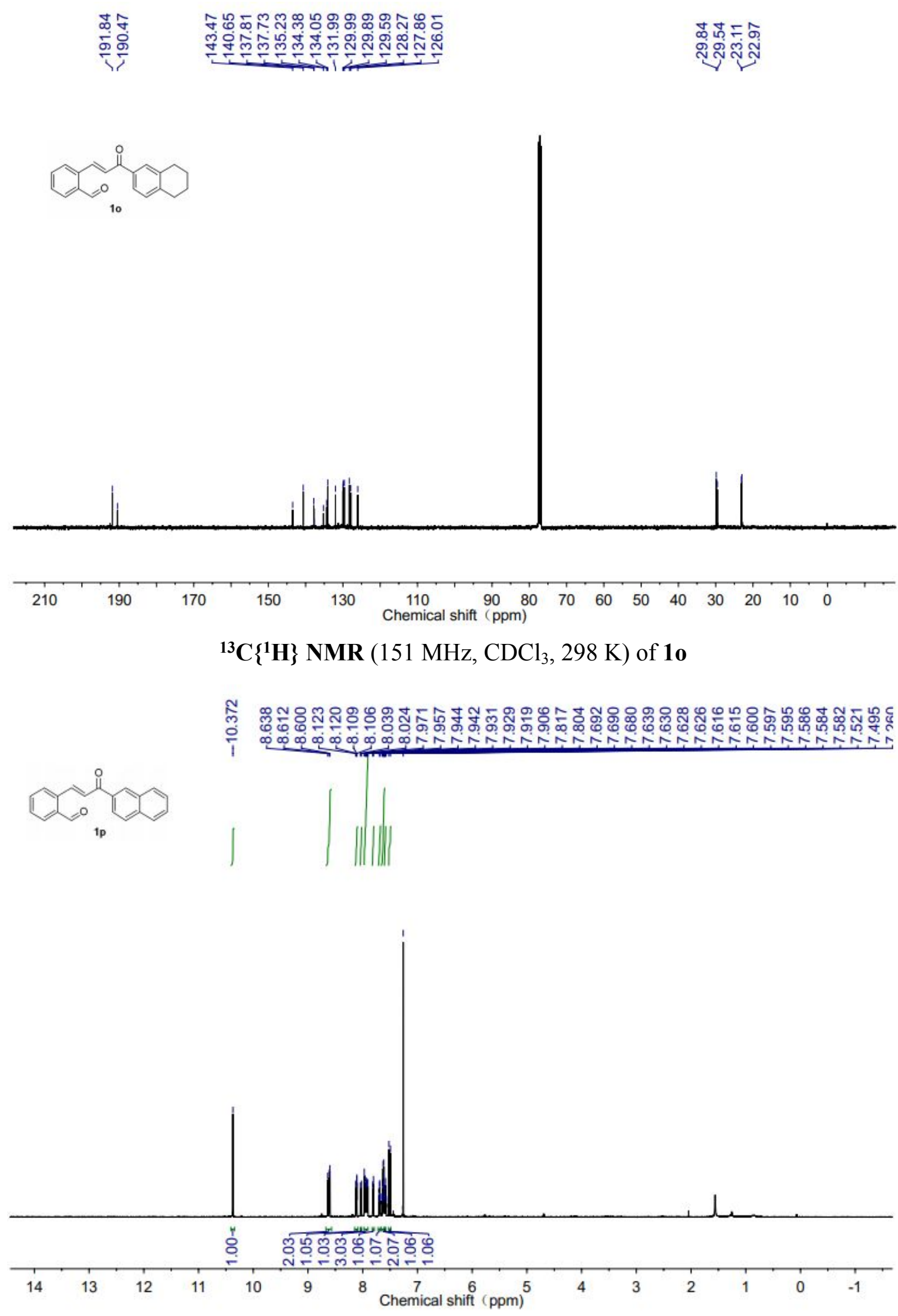


${ }^{1} \mathbf{H}$ NMR $\left(600 \mathrm{MHz}, \mathrm{CDCl}_{3}, 298 \mathrm{~K}\right)$ of $\mathbf{1 p}$
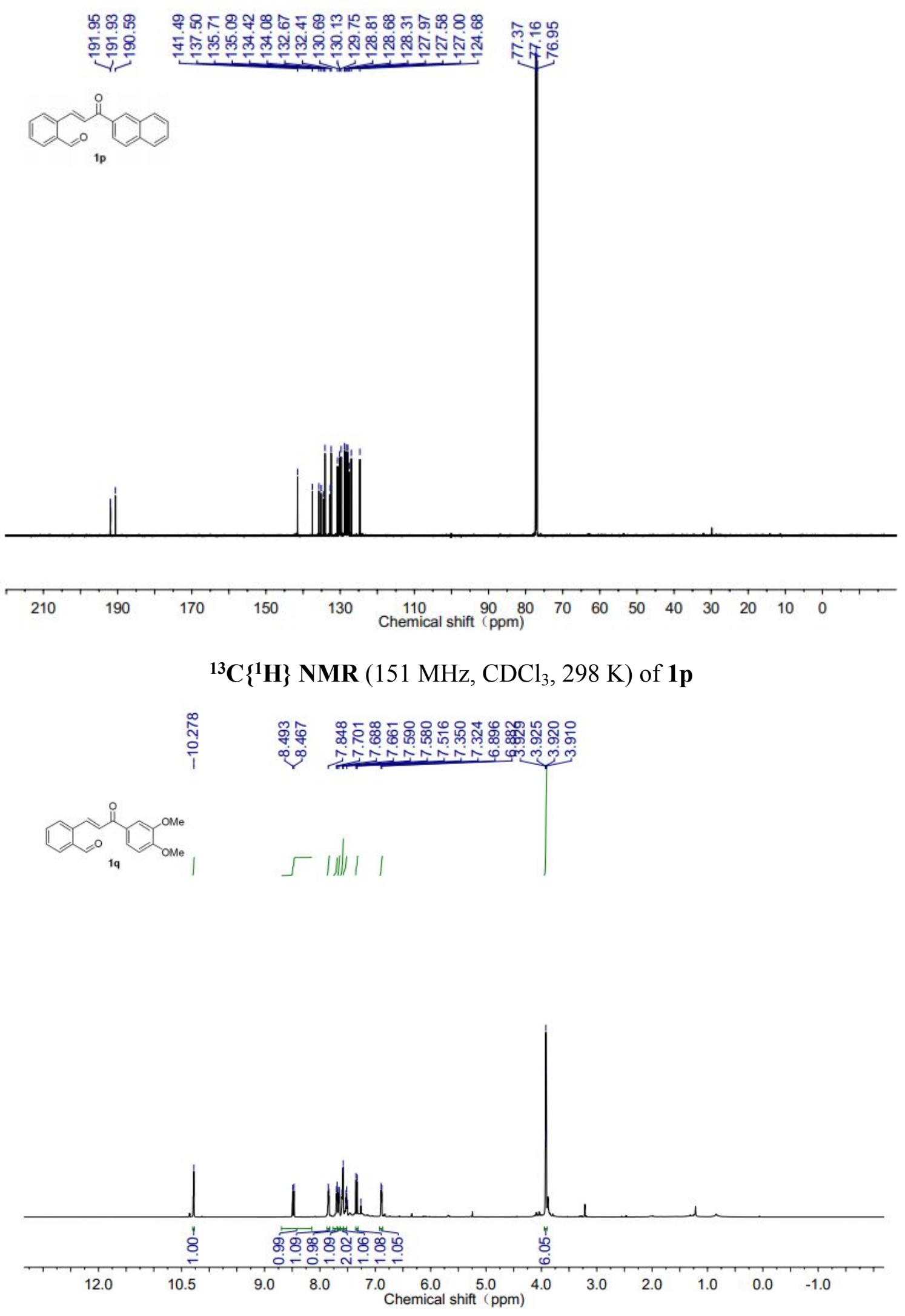
${ }^{1} \mathbf{H}$ NMR $\left(600 \mathrm{MHz}, \mathrm{CDCl}_{3}, 298 \mathrm{~K}\right)$ of $\mathbf{1 q}$
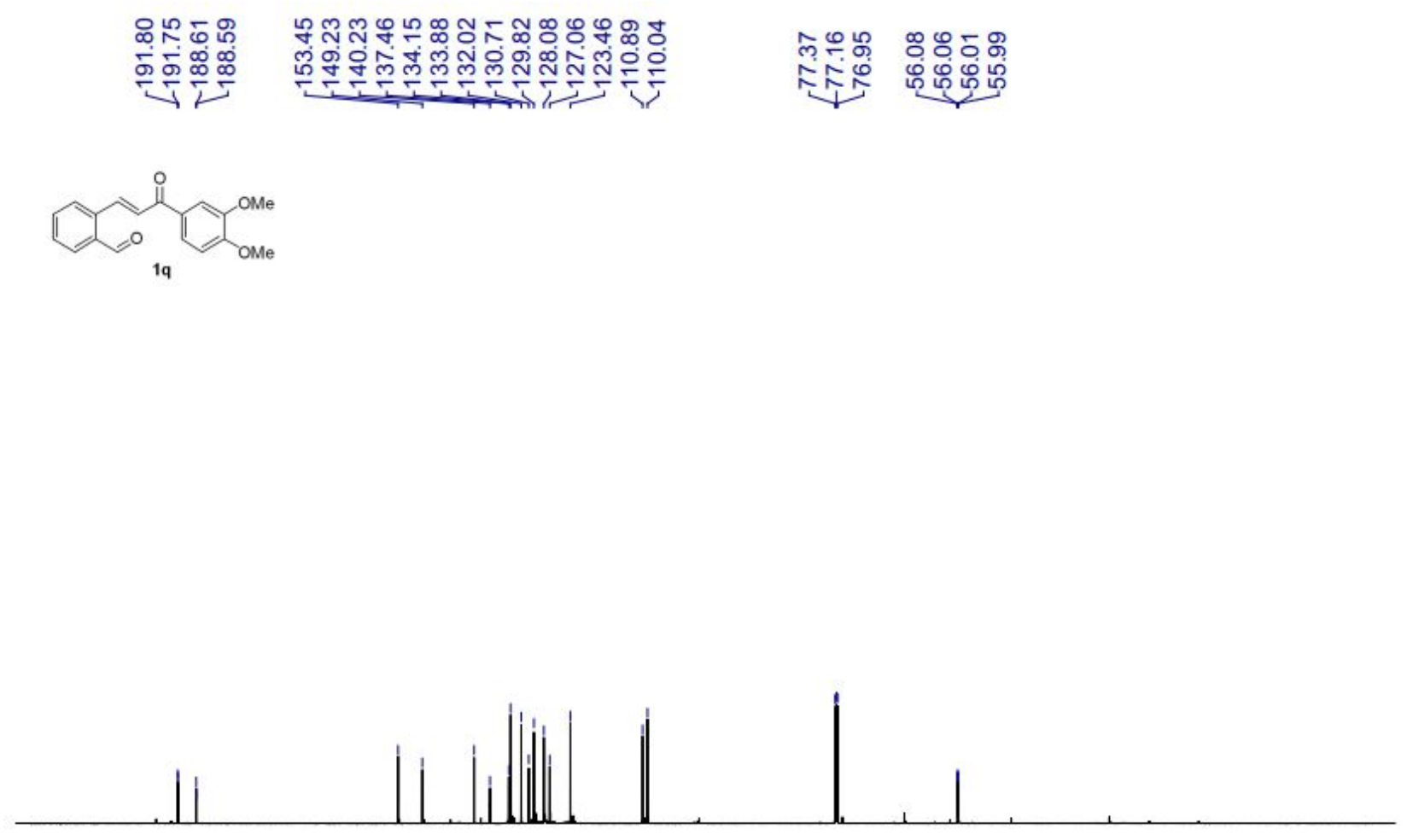

\begin{tabular}{|c|c|c|c|c|c|c|c|c|c|c|c|c|c|c|}
\hline 210 & 190 & 170 & 150 & 130 & 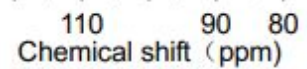 & 70 & 60 & 50 & 40 & 30 & 20 & 10 & 0 & -10 \\
\hline
\end{tabular}


$\overbrace{1}^{\infty} \underbrace{M e}_{M e}$

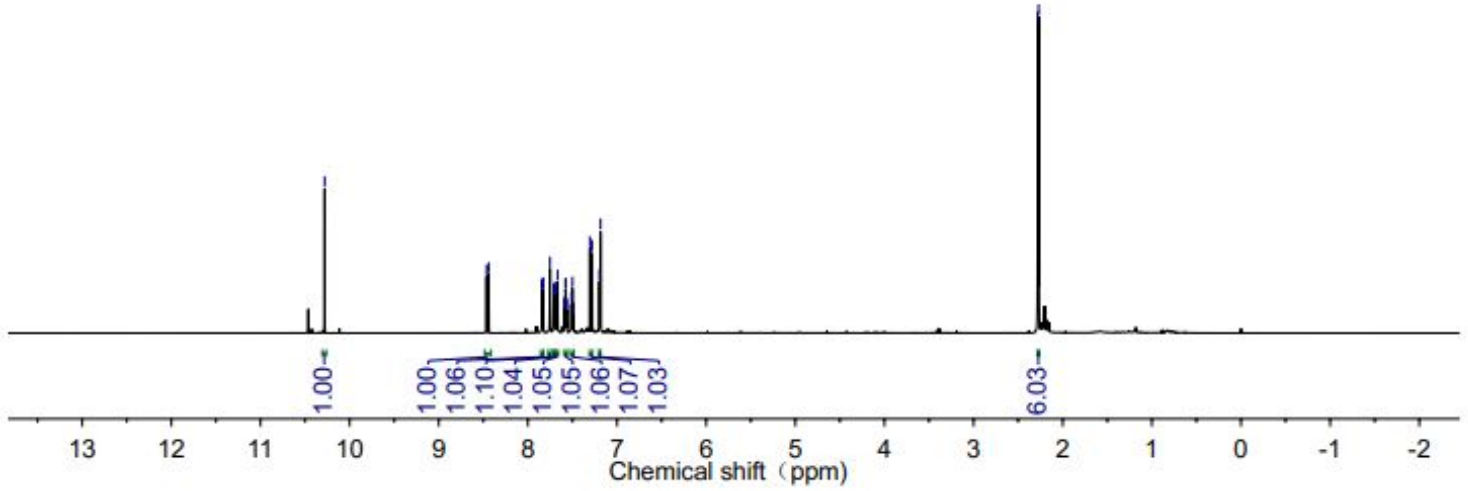

${ }^{1} \mathbf{H}$ NMR $\left(600 \mathrm{MHz}, \mathrm{CDCl}_{3}, 298 \mathrm{~K}\right)$ of $\mathbf{1 r}$
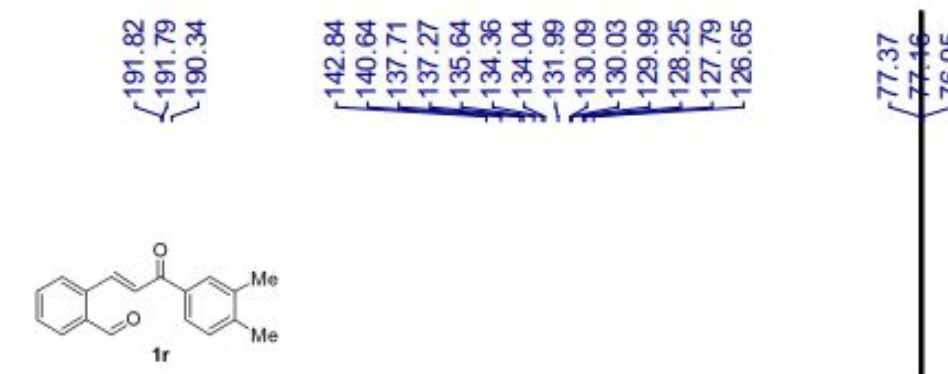

సัฐ
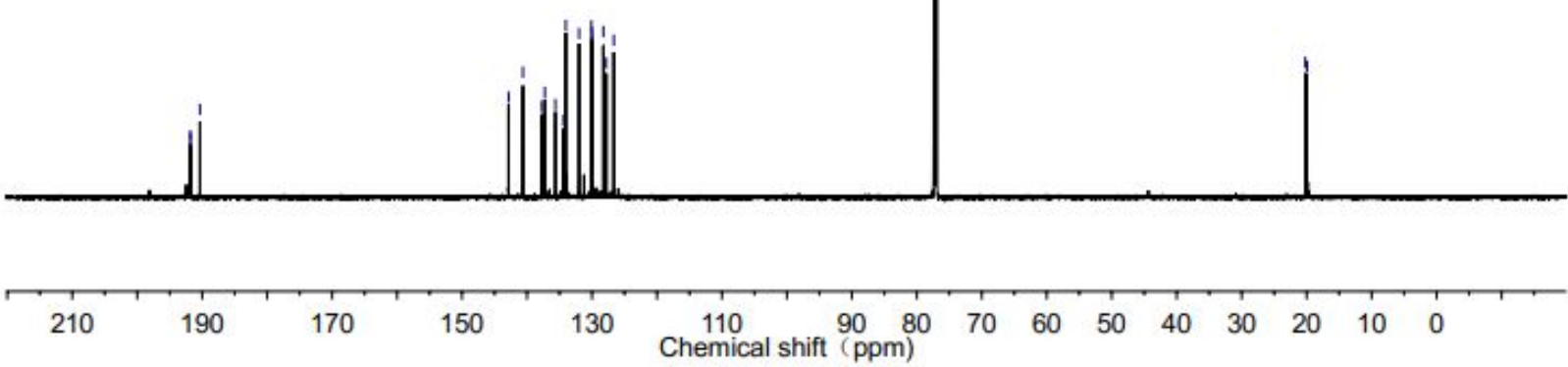

${ }^{13} \mathbf{C}\left\{{ }^{1} \mathbf{H}\right\}$ NMR $\left(151 \mathrm{MHz}, \mathrm{CDCl}_{3}, 298 \mathrm{~K}\right)$ of $\mathbf{1 r}$ 


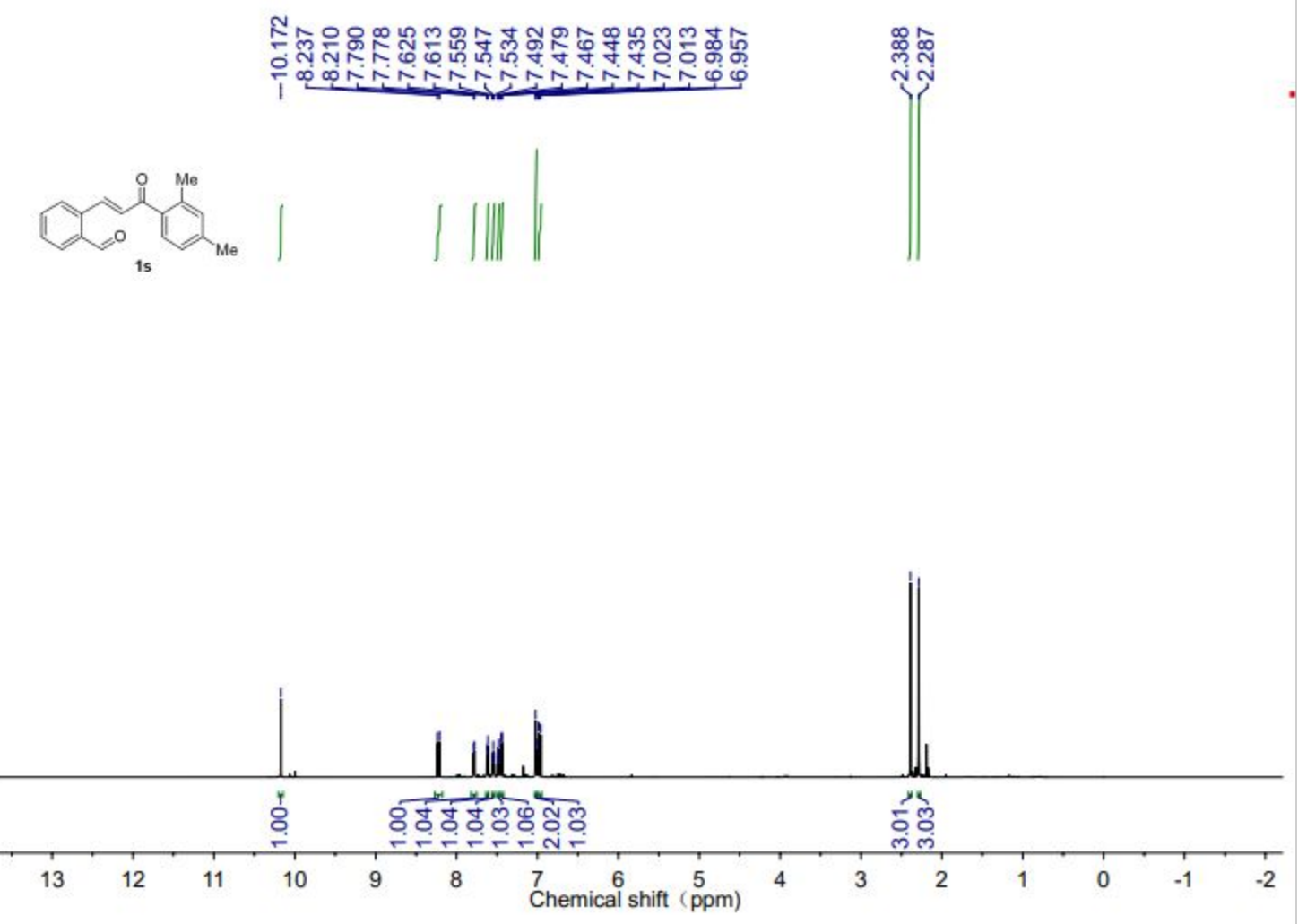

${ }^{1} \mathbf{H}$ NMR $\left(600 \mathrm{MHz}, \mathrm{CDCl}_{3}, 298 \mathrm{~K}\right)$ of $\mathbf{1 s}$

\begin{tabular}{|c|c|c|}
\hline 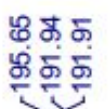 & 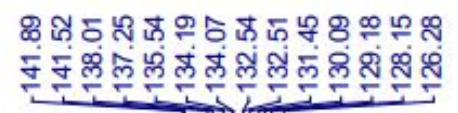 & 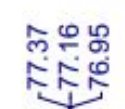 \\
\hline
\end{tabular}
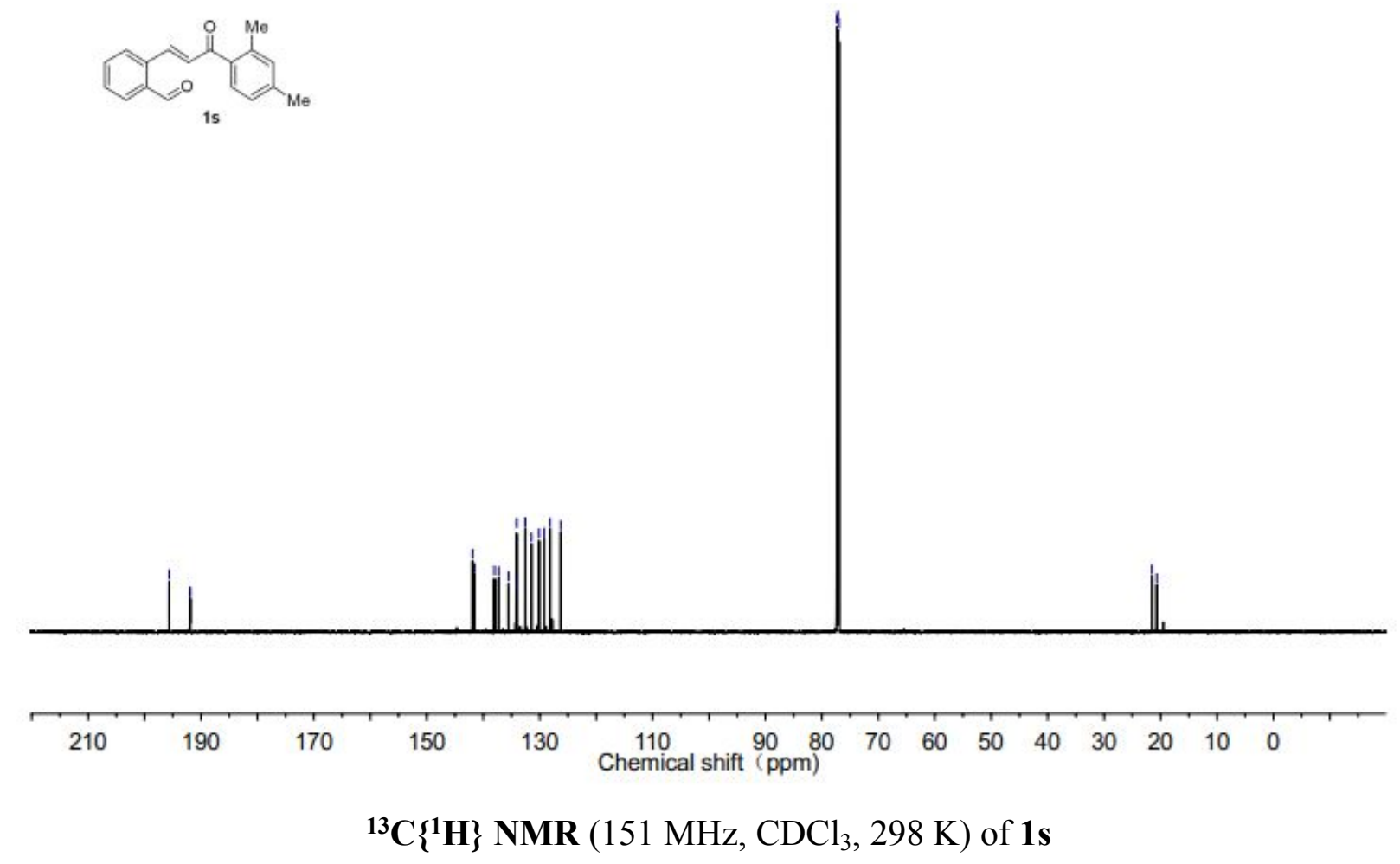

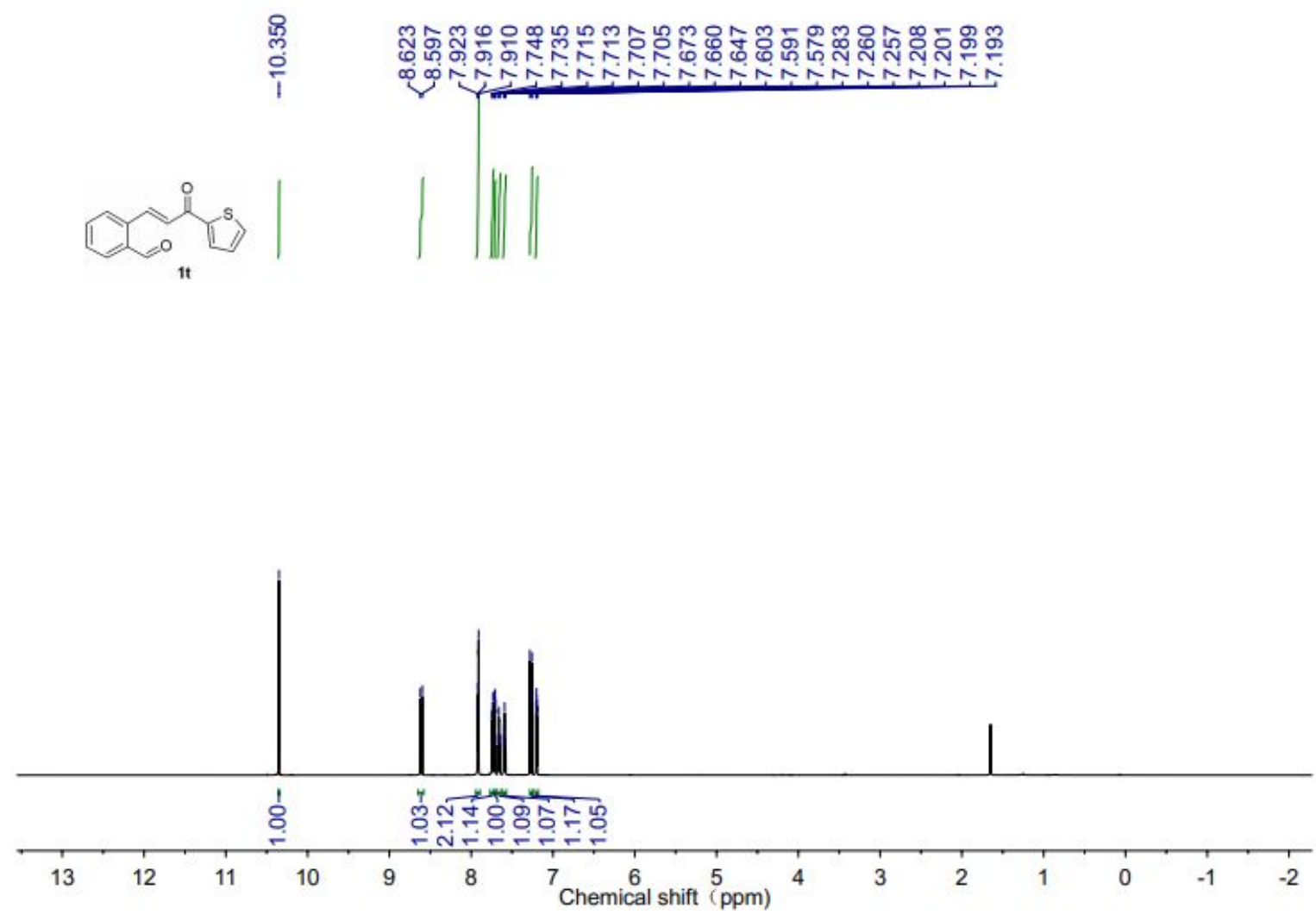

${ }^{1} \mathbf{H}$ NMR $\left(600 \mathrm{MHz}, \mathrm{CDCl}_{3}, 298 \mathrm{~K}\right)$ of $\mathbf{1 t}$

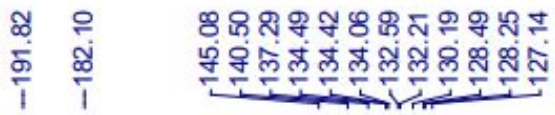

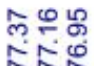
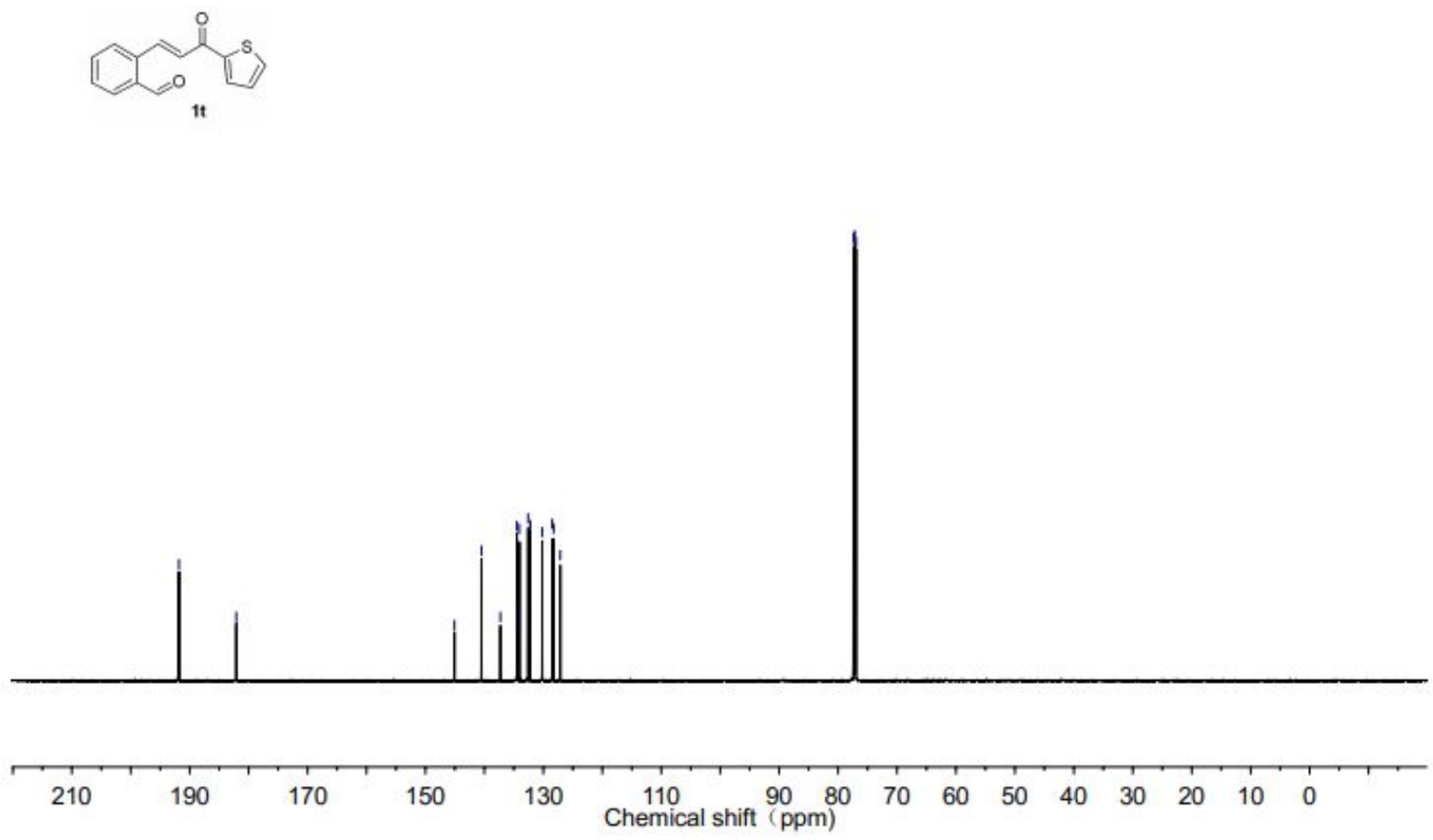

${ }^{13} \mathbf{C}\left\{{ }^{1} \mathbf{H}\right\}$ NMR $\left(151 \mathrm{MHz}, \mathrm{CDCl}_{3}, 298 \mathrm{~K}\right)$ of $\mathbf{1 t}$ 

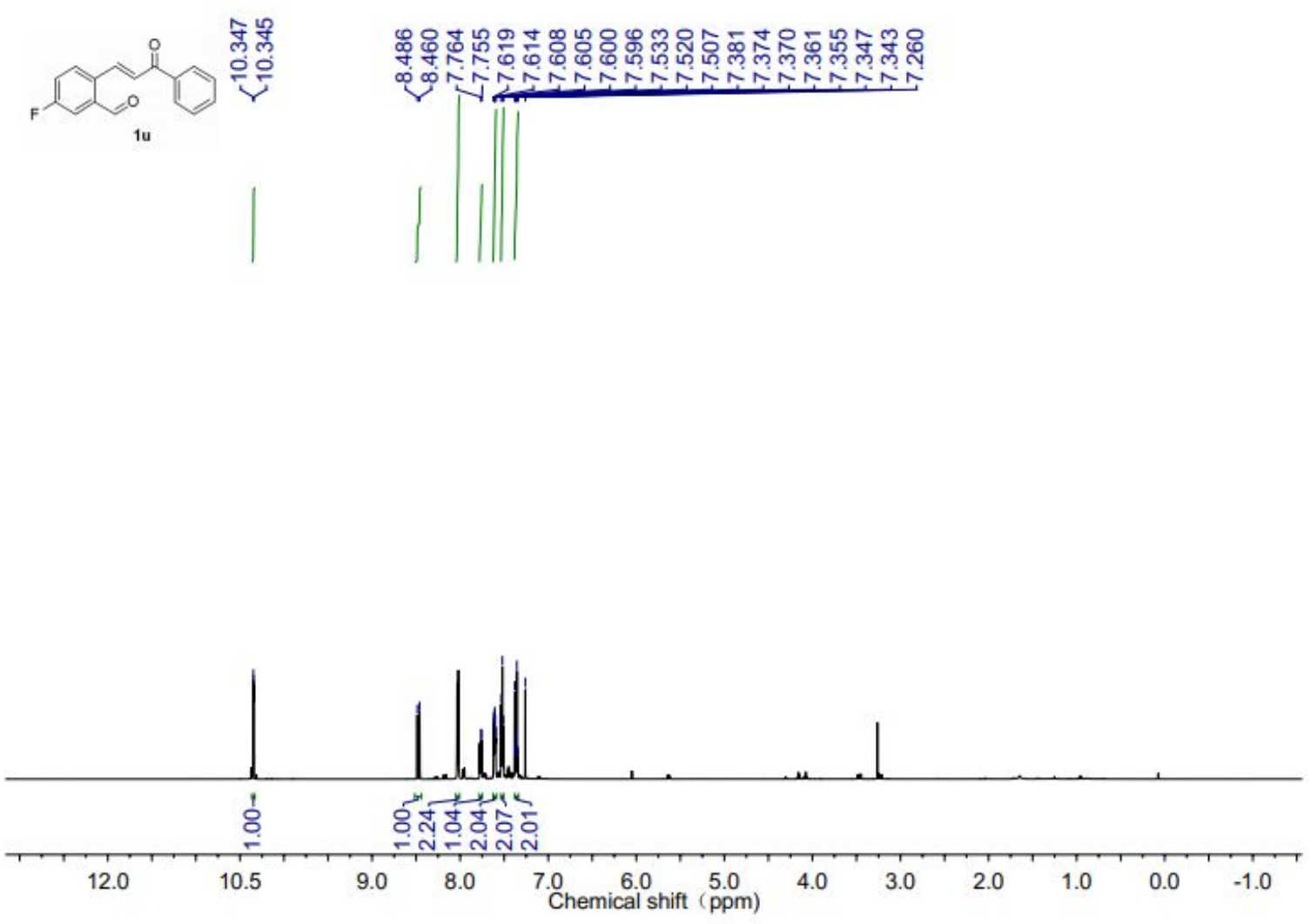

${ }^{1} \mathbf{H}$ NMR $\left(600 \mathrm{MHz}, \mathrm{CDCl}_{3}, 298 \mathrm{~K}\right)$ of $\mathbf{1 u}$

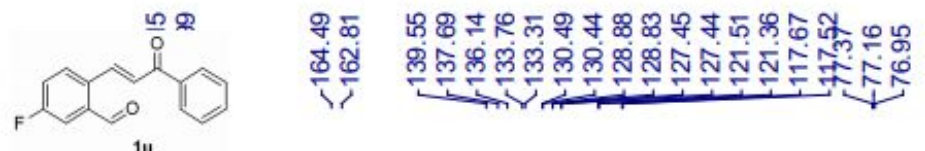

1u

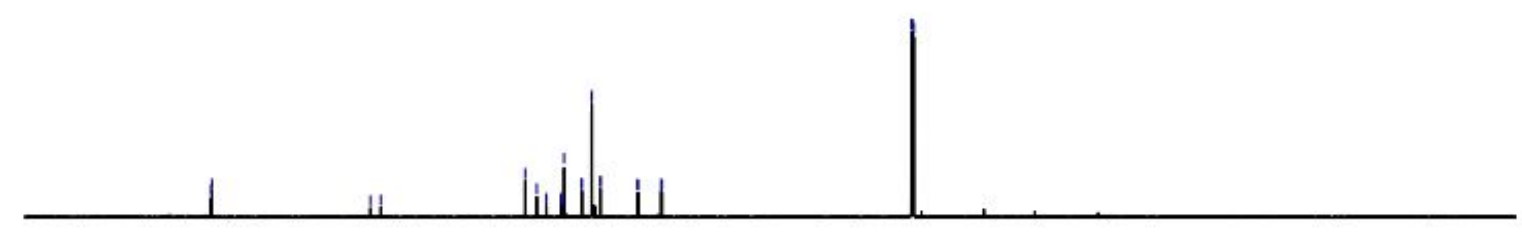

\begin{tabular}{|c|c|c|c|c|c|c|c|c|c|c|c|c|c|}
\hline 210 & 190 & 170 & 150 & 130 & $\begin{array}{c}110 \\
\text { Chemical shift }\end{array} \stackrel{90}{90 m)} 80$ & 70 & 60 & 50 & 40 & 30 & 20 & 10 & 0 \\
\hline
\end{tabular}



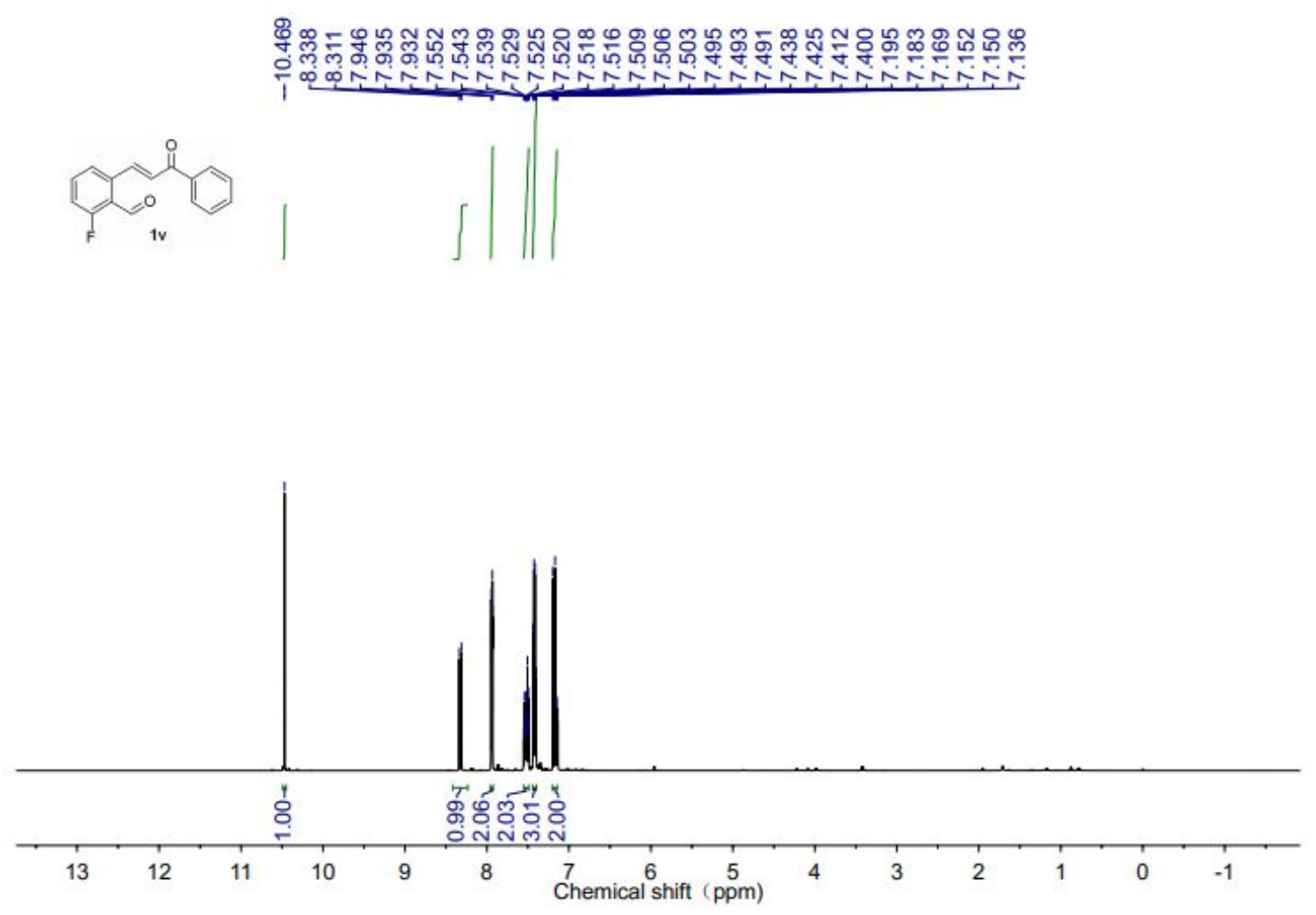

${ }^{1} \mathbf{H}$ NMR $\left(600 \mathrm{MHz}, \mathrm{CDCl}_{3}, 298 \mathrm{~K}\right)$ of $\mathbf{1 v}$

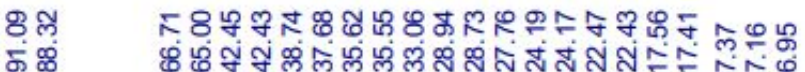

ธั।
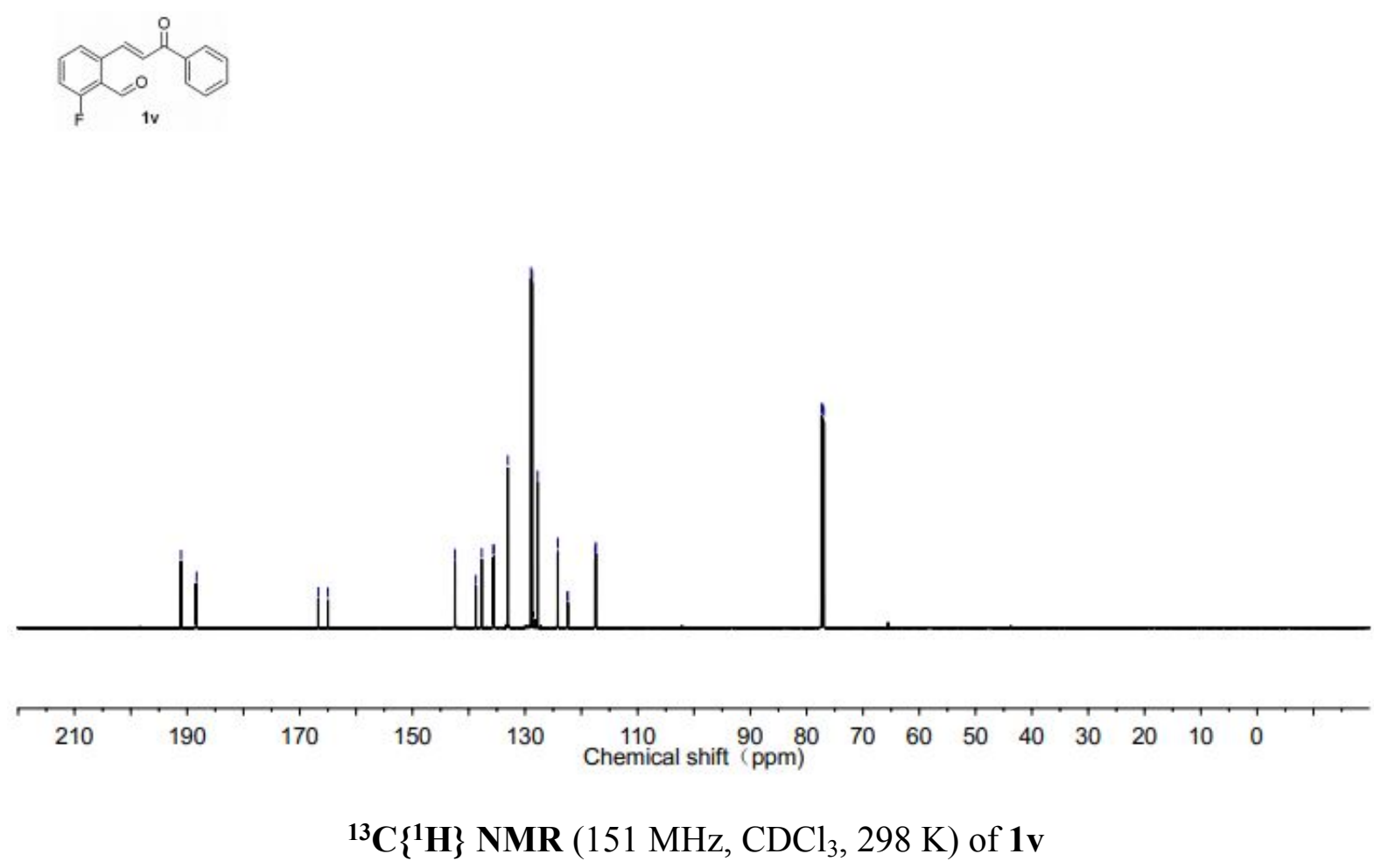

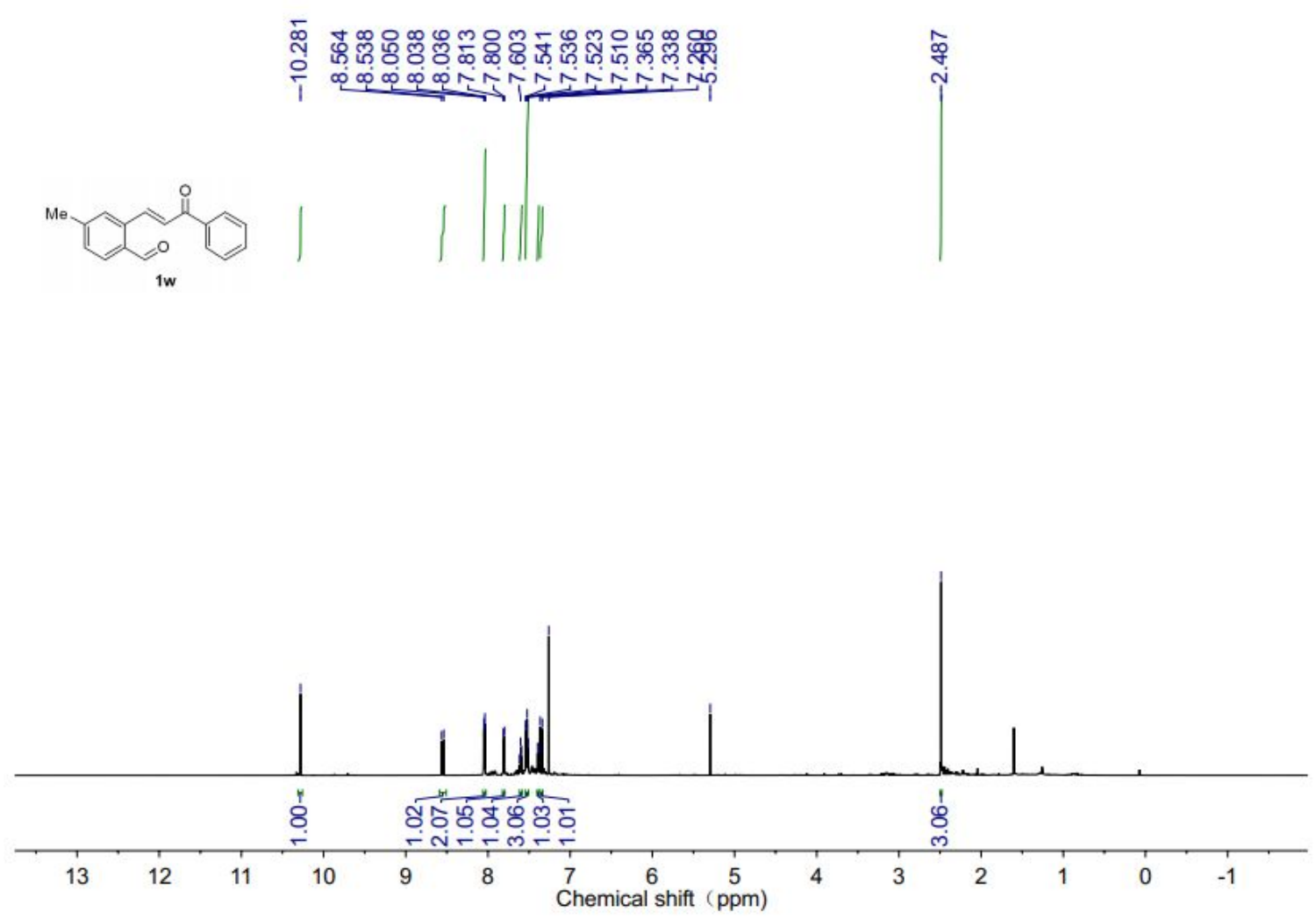

${ }^{1} \mathbf{H}$ NMR $\left(600 \mathrm{MHz}, \mathrm{CDCl}_{3}, 298 \mathrm{~K}\right)$ of $\mathbf{1 w}$

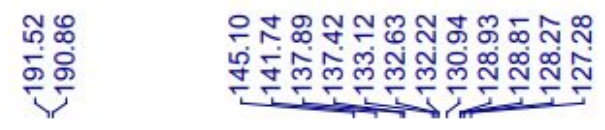

笑是怘

$\stackrel{\hat{\tau}}{\grave{T}}$
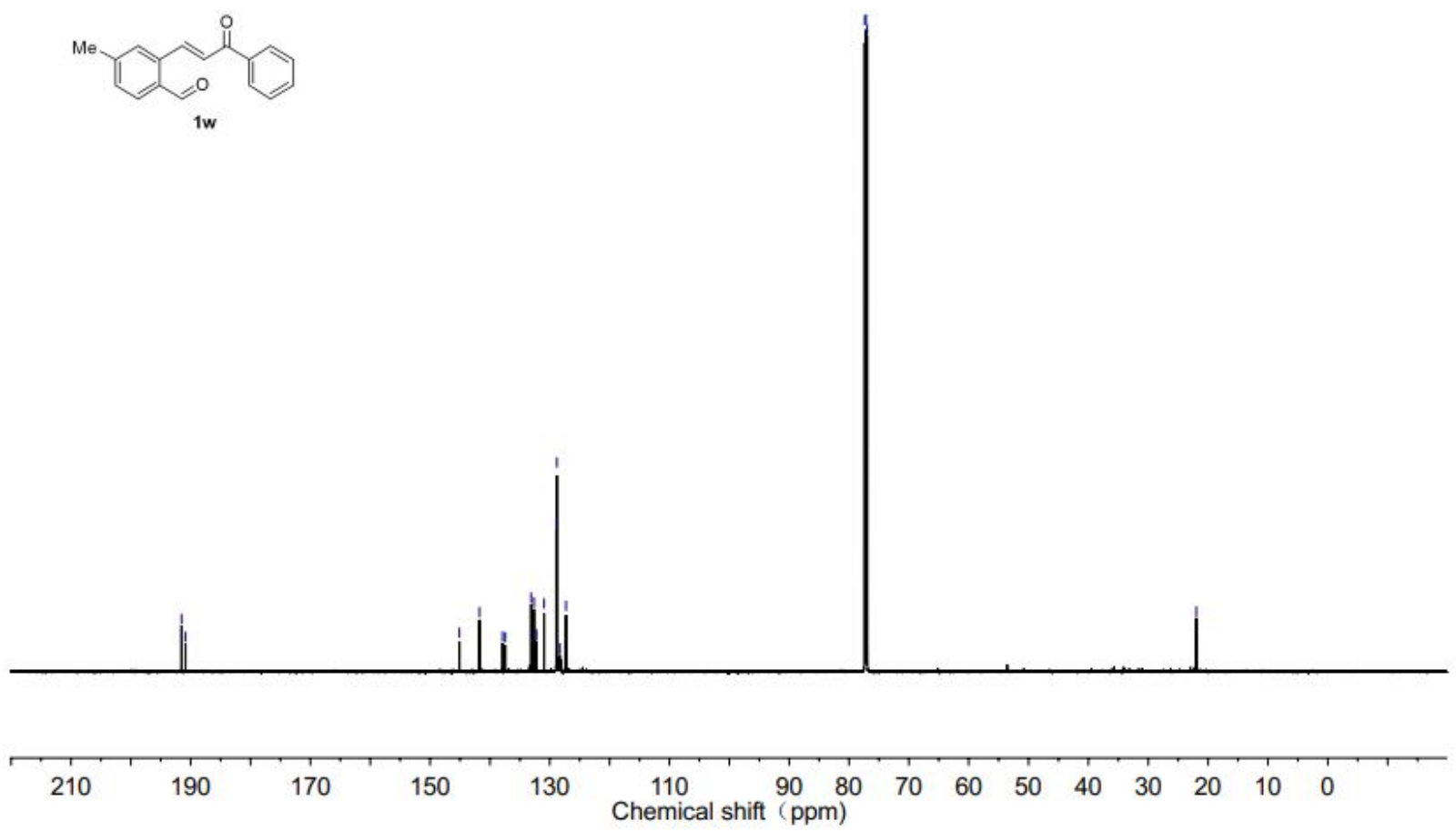

${ }^{13} \mathbf{C}\left\{{ }^{1} \mathbf{H}\right\}$ NMR $\left(151 \mathrm{MHz}, \mathrm{CDCl}_{3}, 298 \mathrm{~K}\right)$ of $\mathbf{1 w}$

64 


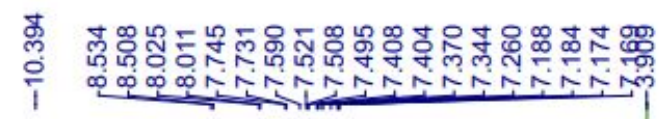
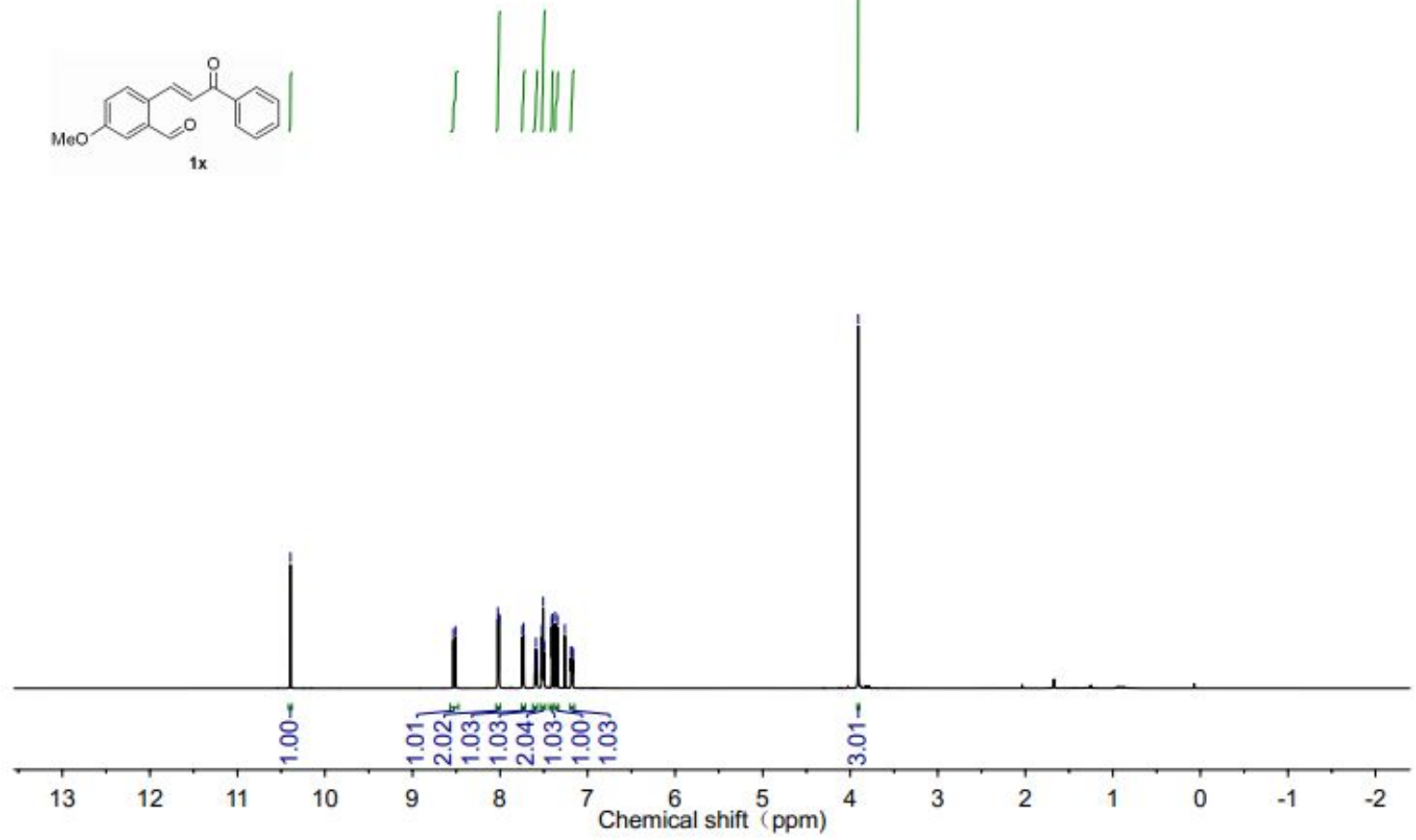

${ }^{1} \mathbf{H}$ NMR $\left(600 \mathrm{MHz}, \mathrm{CDCl}_{3}, 298 \mathrm{~K}\right)$ of $\mathbf{1 x}$

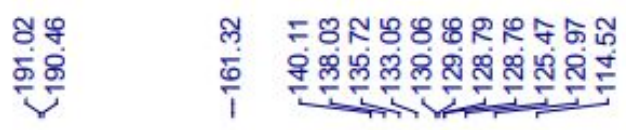

慜曼
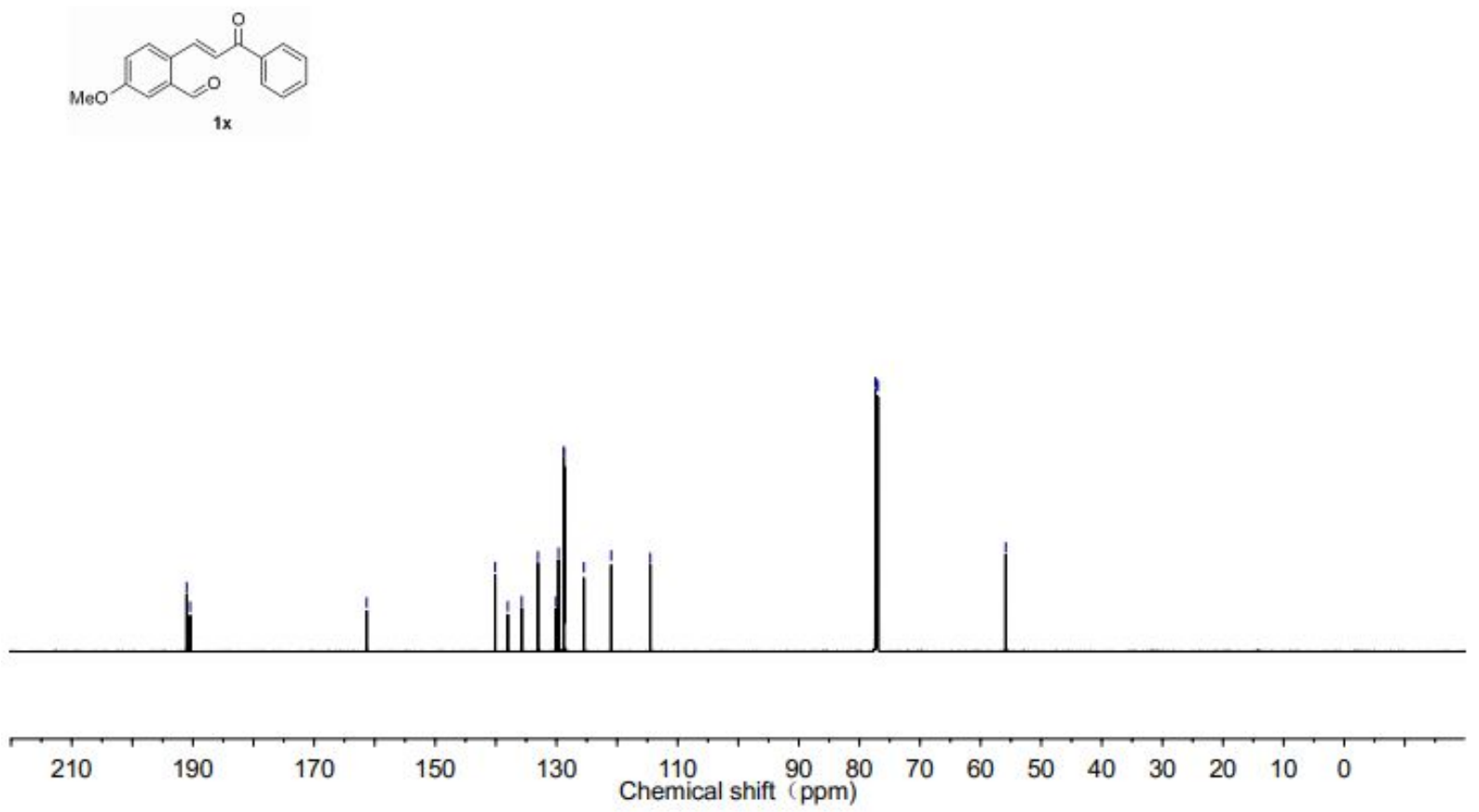

${ }^{13} \mathbf{C}\left\{{ }^{1} \mathbf{H}\right\}$ NMR (151 MHz, $\left.\mathrm{CDCl}_{3}, 298 \mathrm{~K}\right)$ of $\mathbf{1 x}$

65 

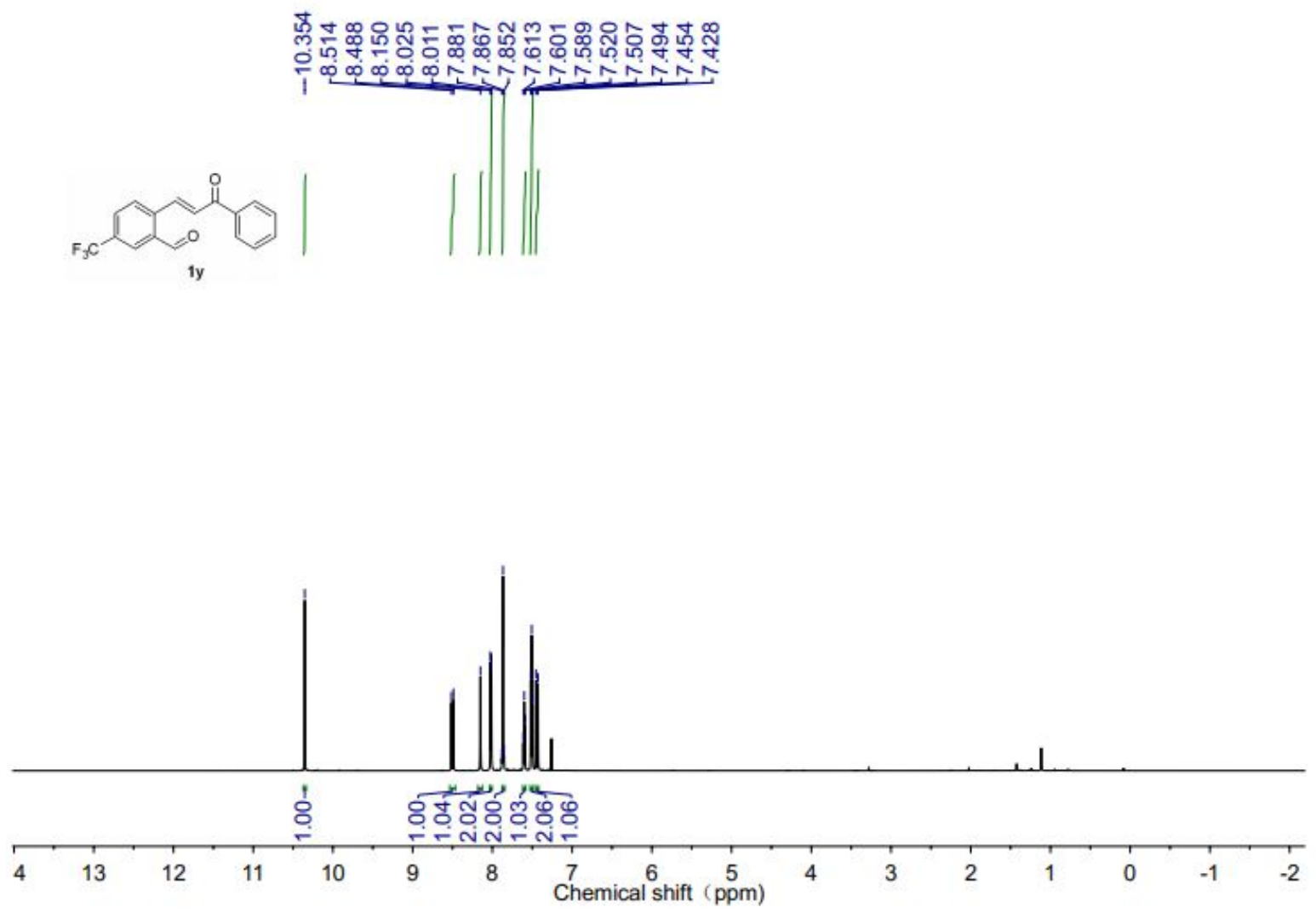

${ }^{1} \mathbf{H}$ NMR $\left(600 \mathrm{MHz}, \mathrm{CDCl}_{3}, 298 \mathrm{~K}\right)$ of $\mathbf{1 y}$

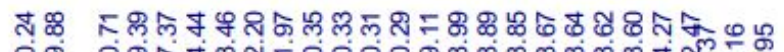

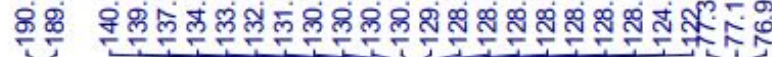
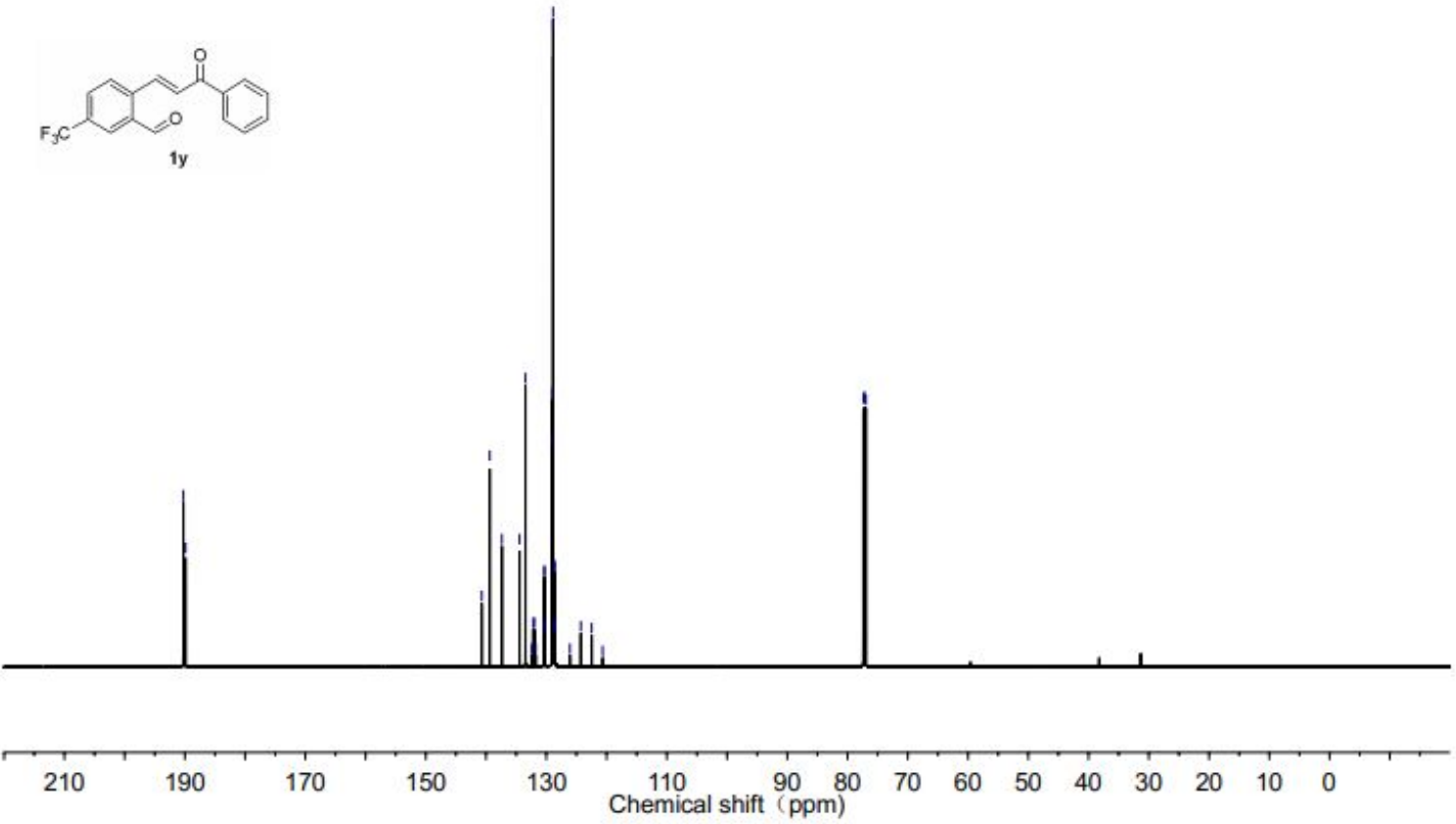

${ }^{13} \mathbf{C}\left\{{ }^{1} \mathbf{H}\right\}$ NMR $\left(151 \mathrm{MHz}, \mathrm{CDCl}_{3}, 298 \mathrm{~K}\right)$ of $\mathbf{1 y}$ 

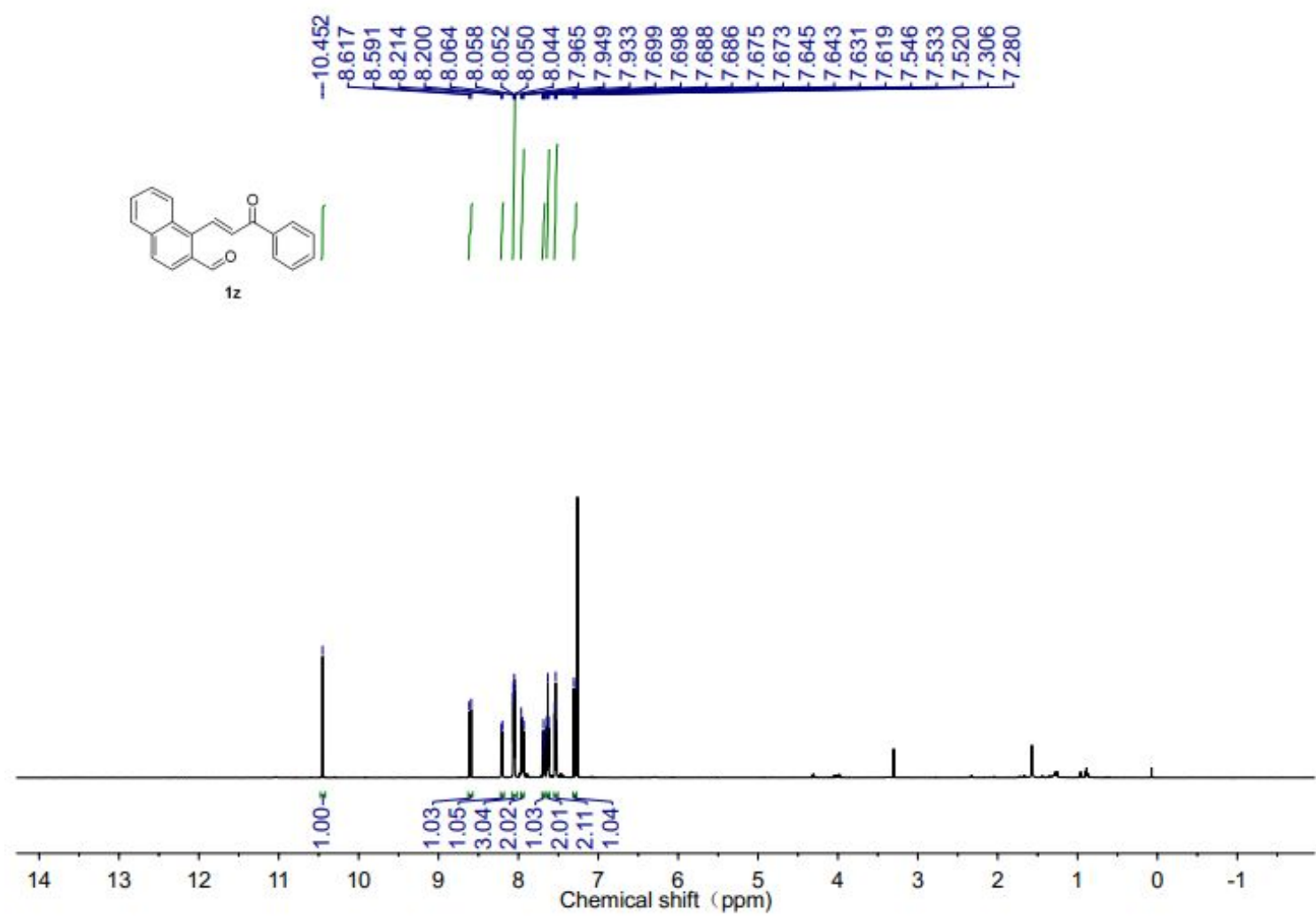

${ }^{1} \mathbf{H}$ NMR $\left(600 \mathrm{MHz}, \mathrm{CDCl}_{3}, 298 \mathrm{~K}\right)$ of $\mathbf{1 z}$
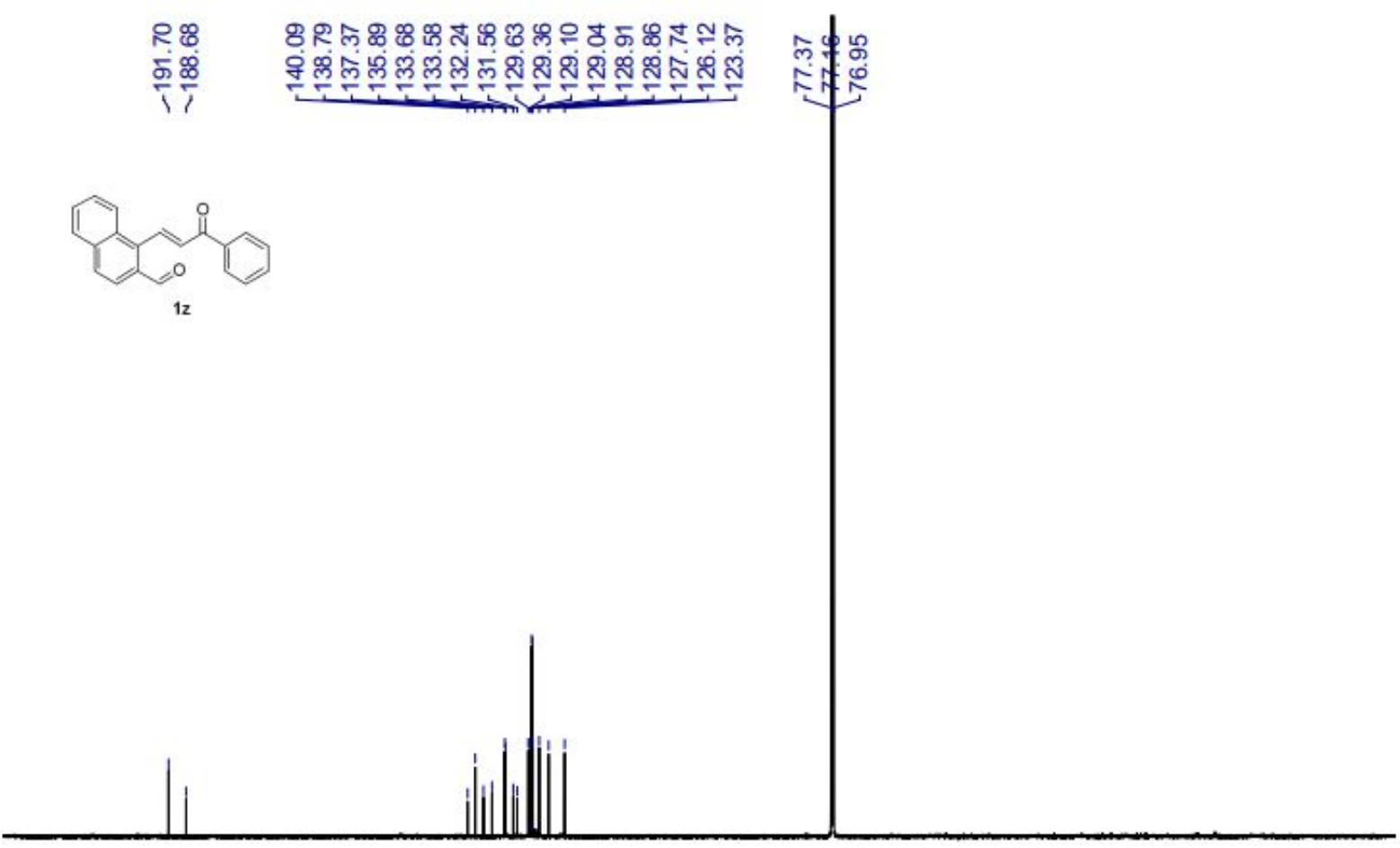

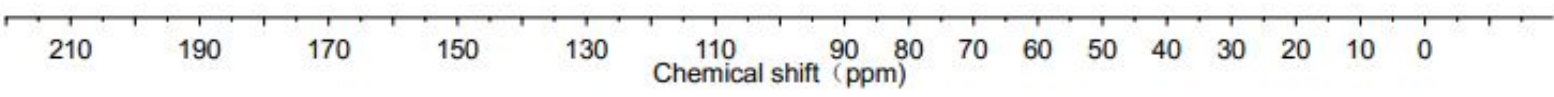


${ }^{13} \mathbf{C}\left\{{ }^{1} \mathbf{H}\right\}$ NMR (151 MHz, $\left.\mathrm{CDCl}_{3}, 298 \mathrm{~K}\right)$ of $\mathbf{1 z}$
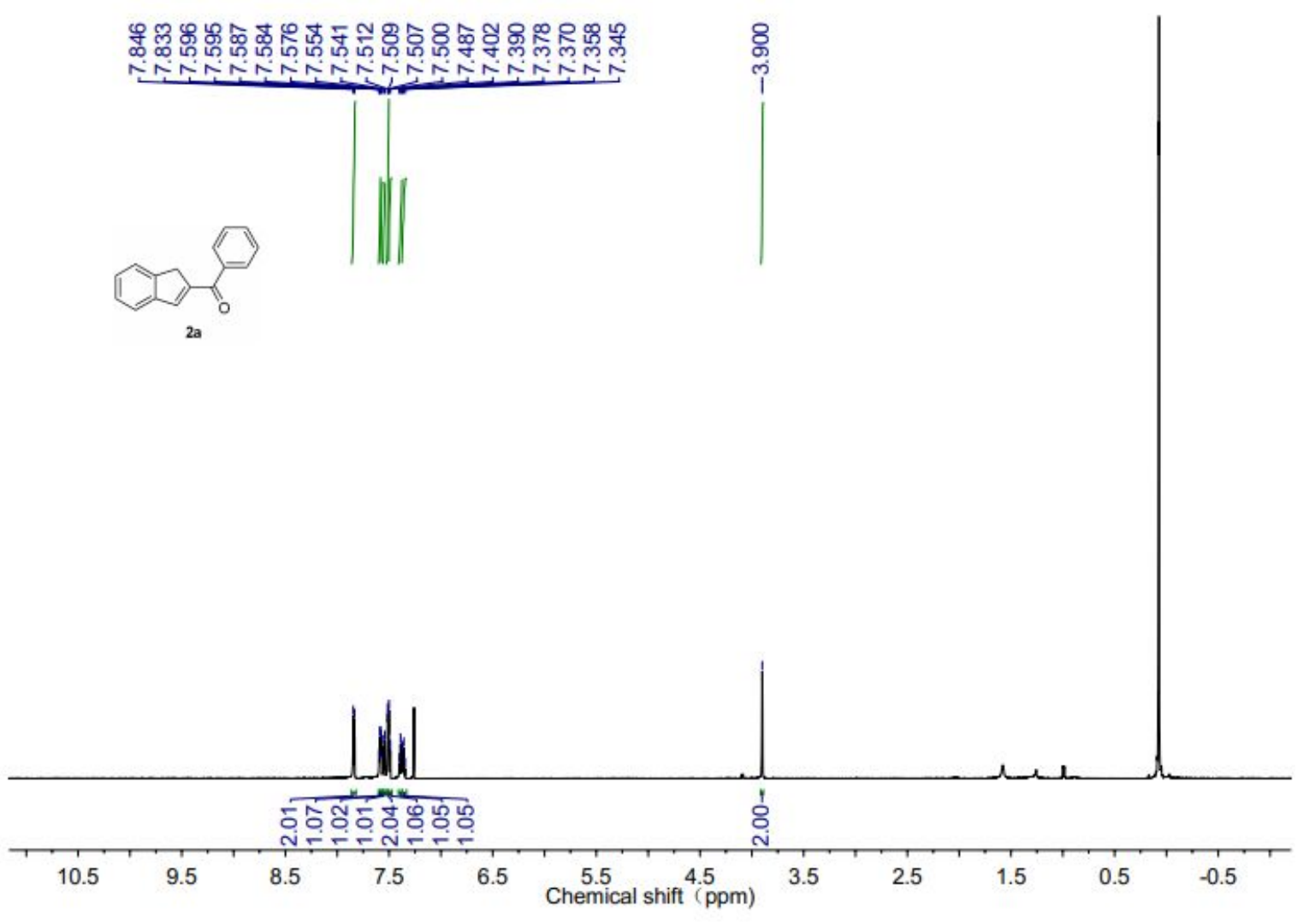

${ }^{1} \mathbf{H}$ NMR $\left(600 \mathrm{MHz}, \mathrm{CDCl}_{3}, 298 \mathrm{~K}\right)$ of $\mathbf{2 a}$ 


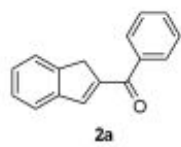

2a
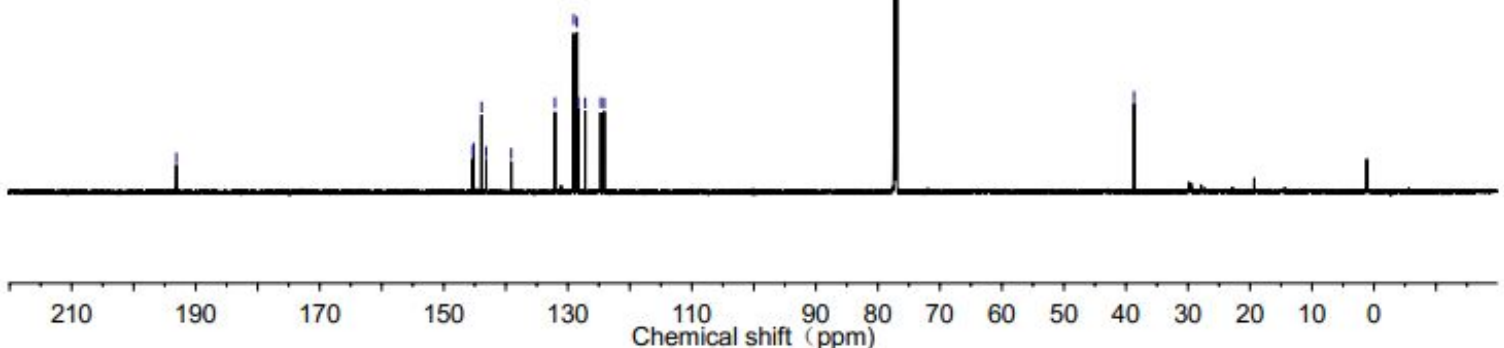

${ }^{13} \mathbf{C}\left\{{ }^{1} \mathbf{H}\right\}$ NMR $\left(151 \mathrm{MHz}, \mathrm{CDCl}_{3}, 298 \mathrm{~K}\right)$ of $\mathbf{2 a}$

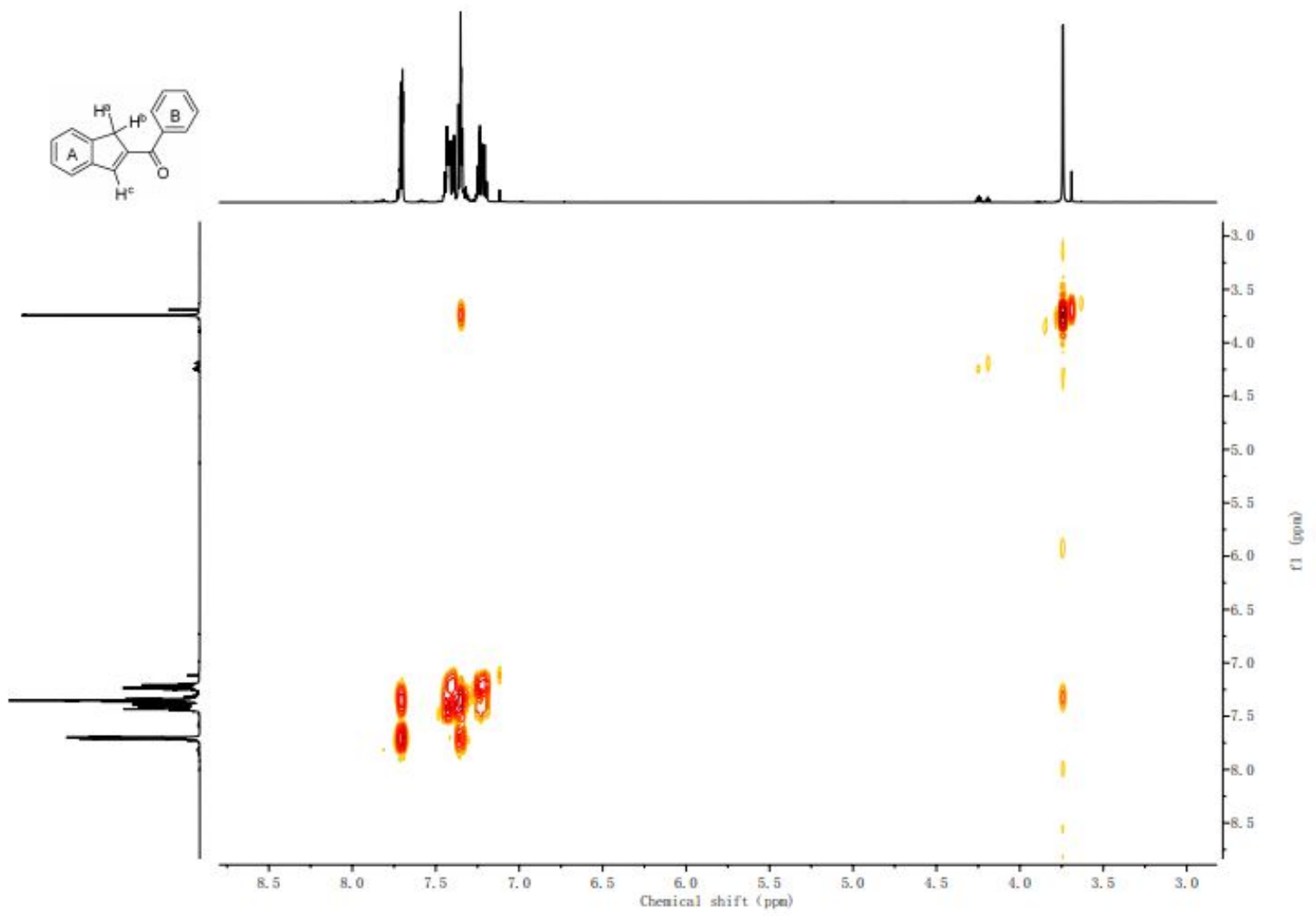


${ }^{1} \mathrm{H}^{-1} \mathrm{H}$ COSY of $\mathbf{2 a}$

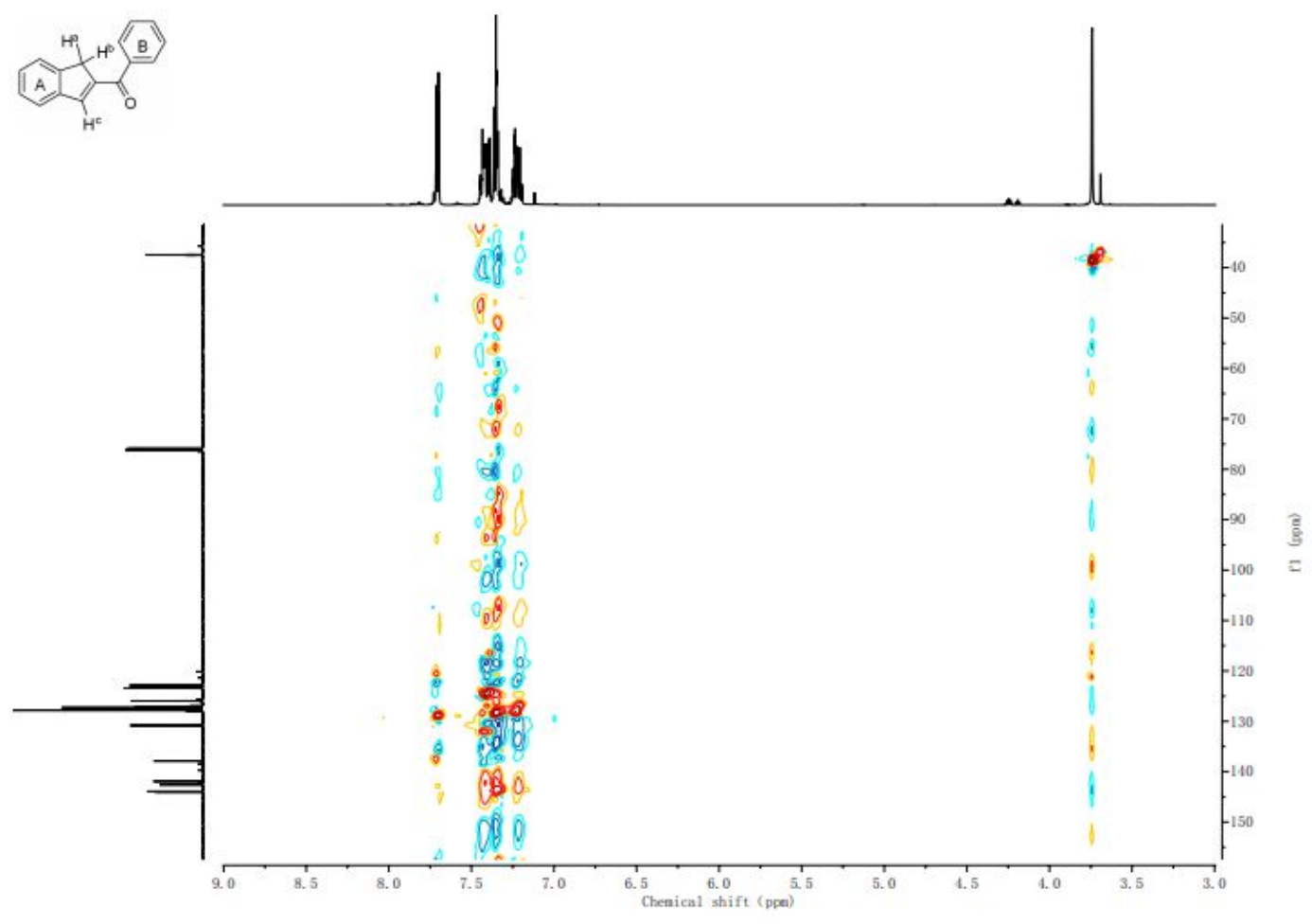

${ }^{1} \mathrm{H}-{ }^{13} \mathrm{C}$ HSQC of $2 \mathrm{a}$

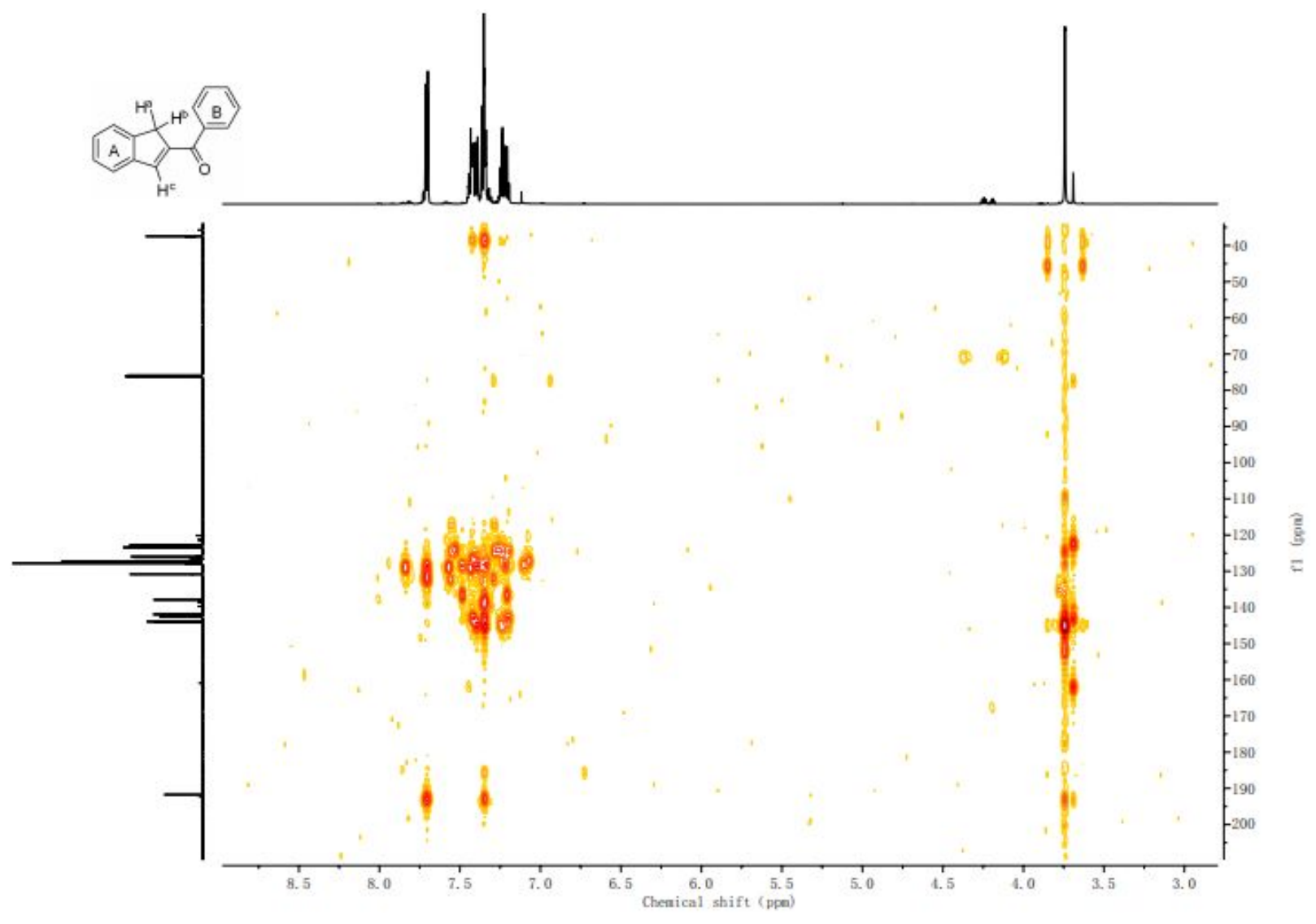


${ }^{1} \mathrm{H}-{ }^{13} \mathrm{C}$ HMBC of $2 \mathrm{a}$

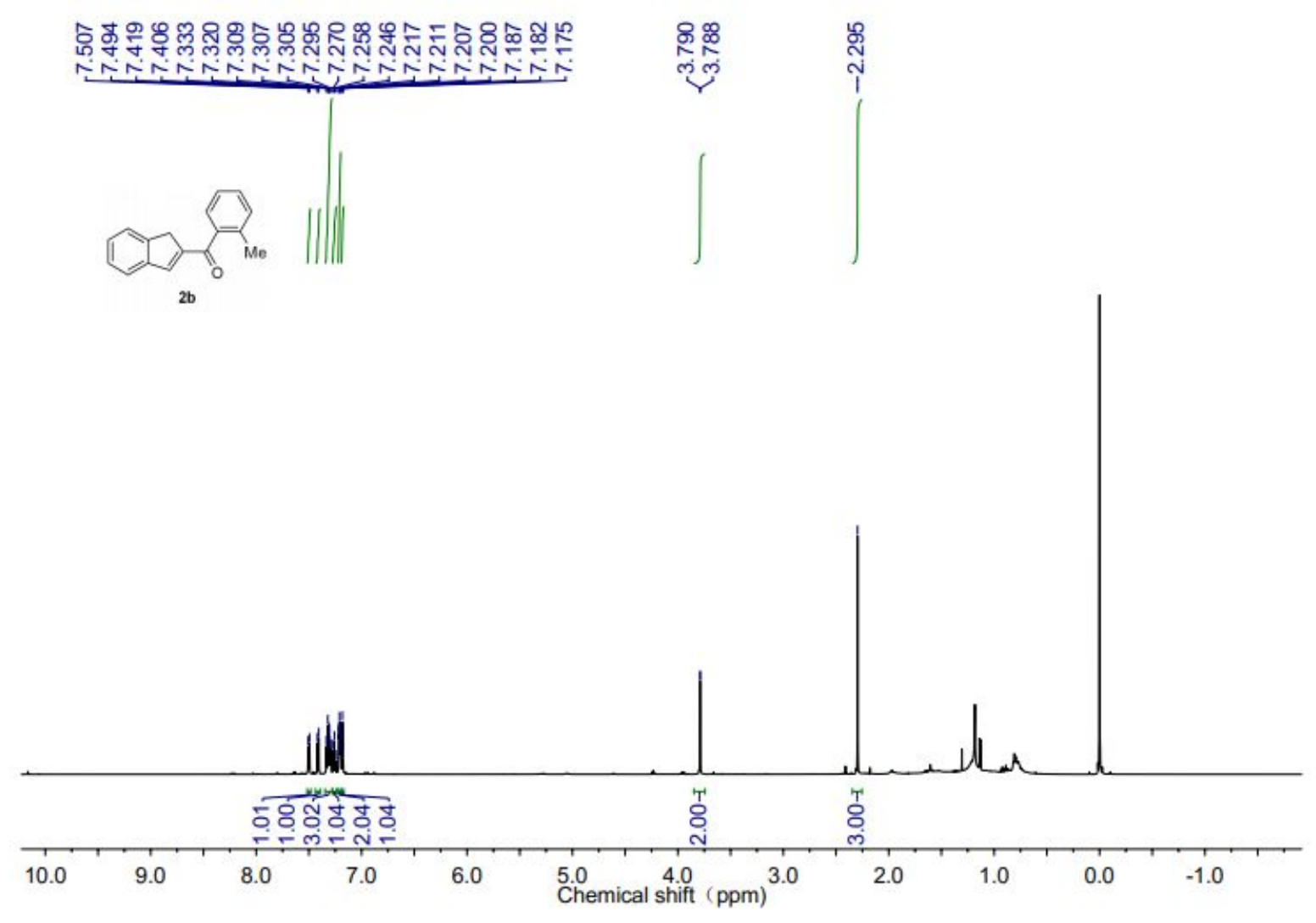

${ }^{\mathbf{1}} \mathbf{H}$ NMR (600 MHz, $\left.\mathrm{CDCl}_{3}, 298 \mathrm{~K}\right)$ of $\mathbf{2 b}$ 


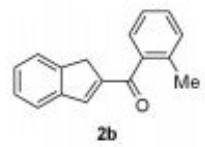

2b
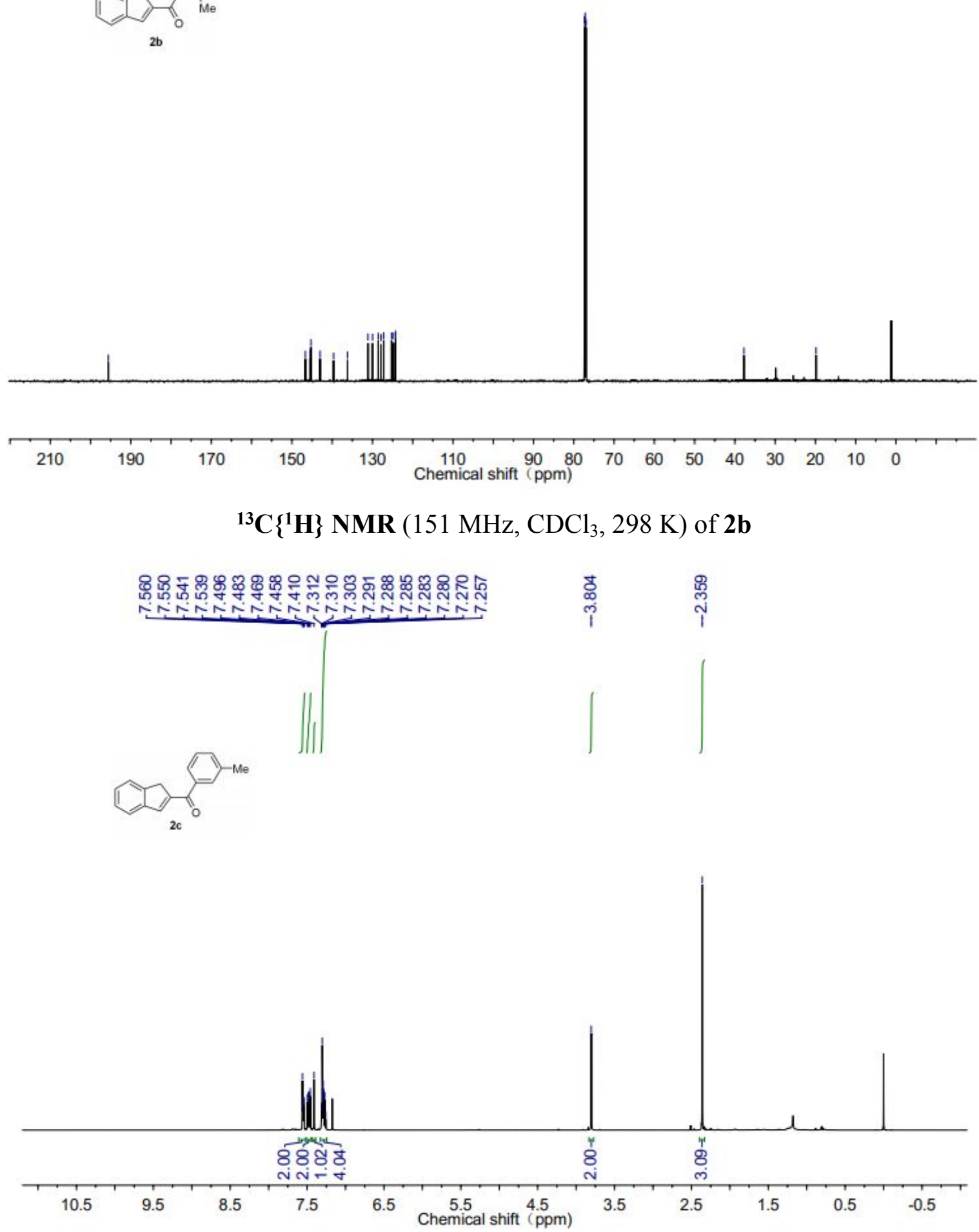

${ }^{1} \mathbf{H}$ NMR $\left(600 \mathrm{MHz}, \mathrm{CDCl}_{3}, 298 \mathrm{~K}\right)$ of $\mathbf{2 c}$ 

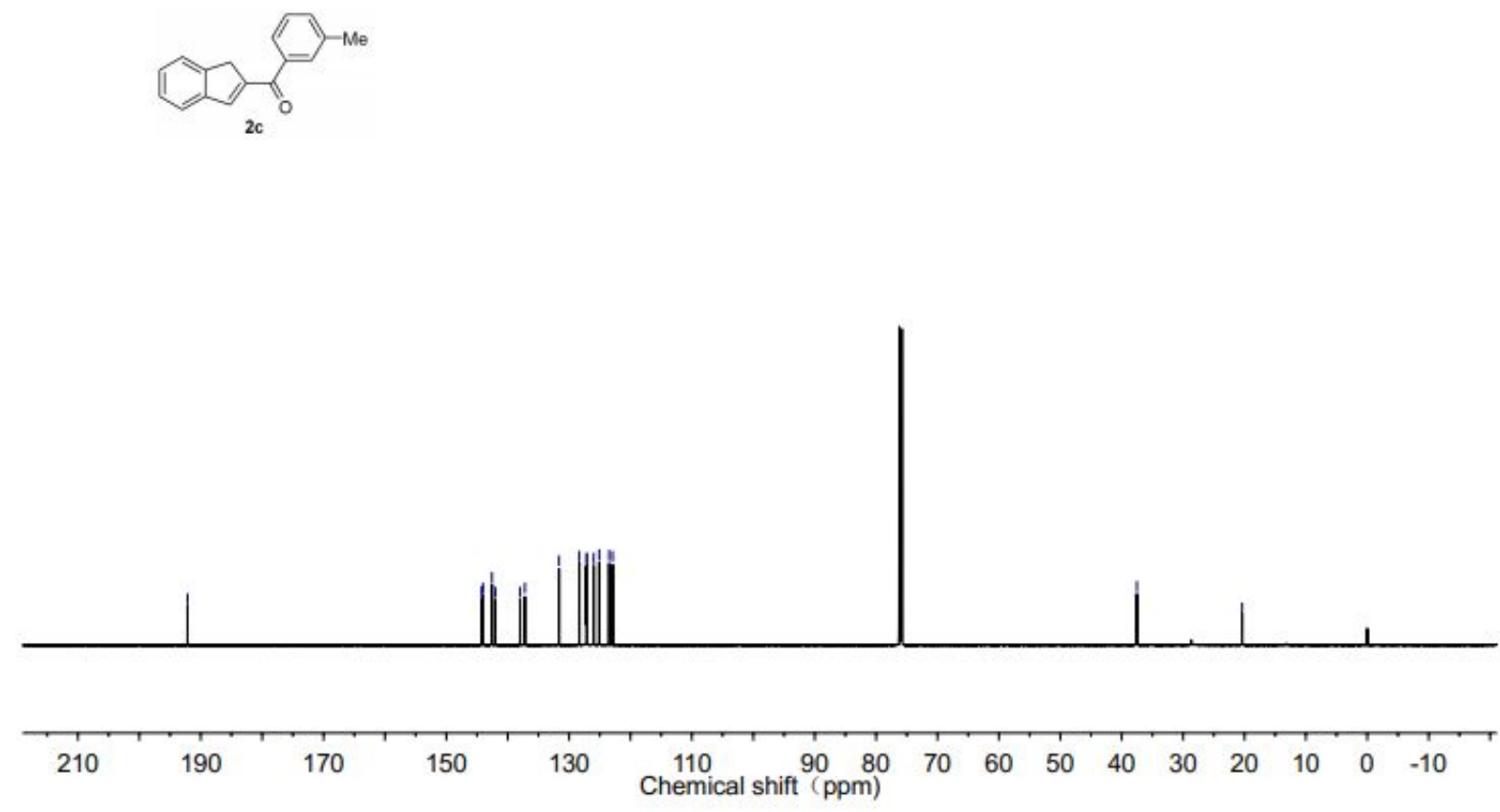

${ }^{13} \mathbf{C}\left\{{ }^{1} \mathbf{H}\right\}$ NMR $\left(151 \mathrm{MHz}, \mathrm{CDCl}_{3}, 298 \mathrm{~K}\right)$ of $\mathbf{2 c}$

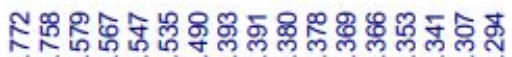

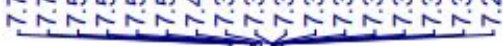
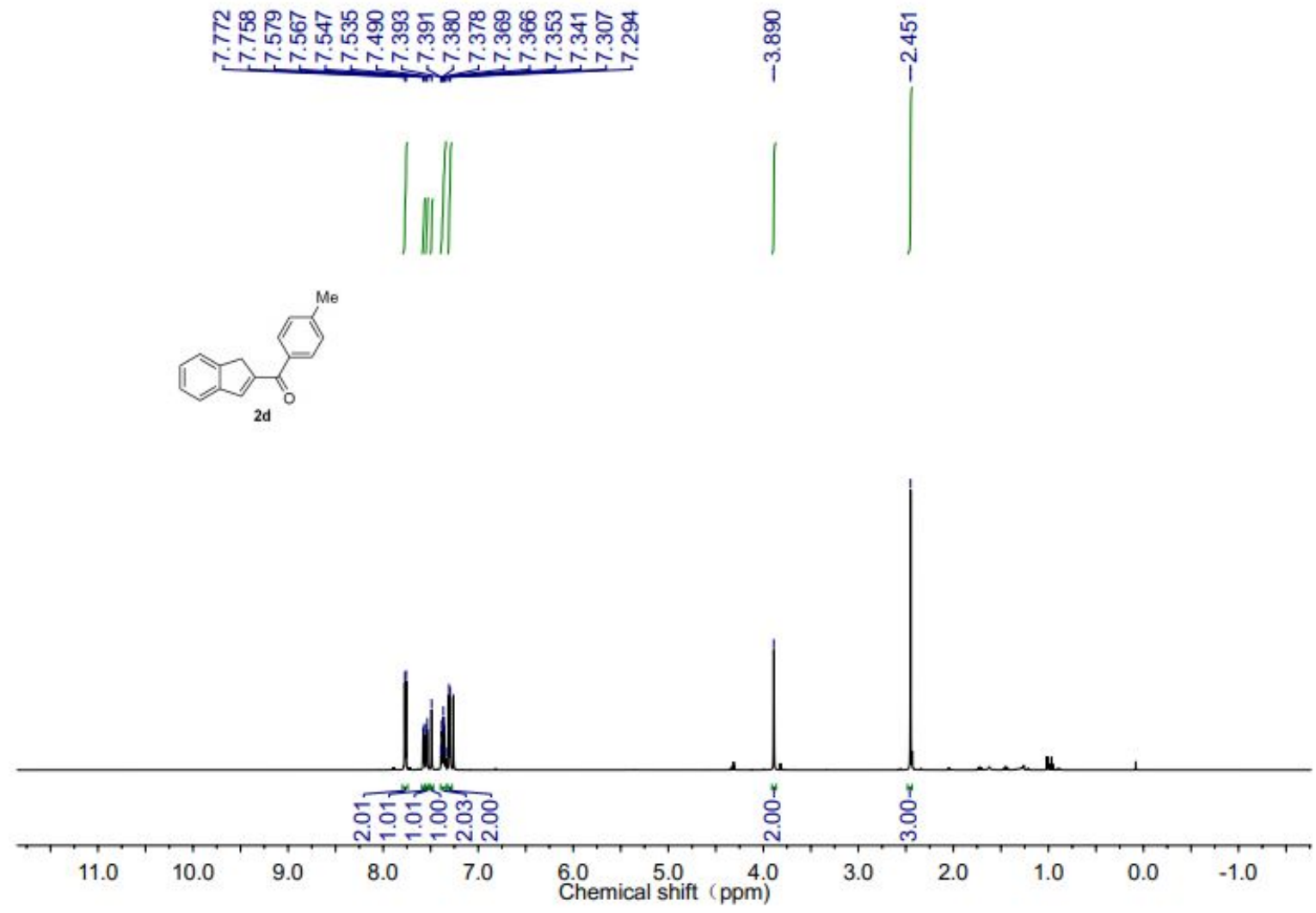

${ }^{1} \mathbf{H}$ NMR $\left(600 \mathrm{MHz}, \mathrm{CDCl}_{3}, 298 \mathrm{~K}\right)$ of $\mathbf{2 d}$ 

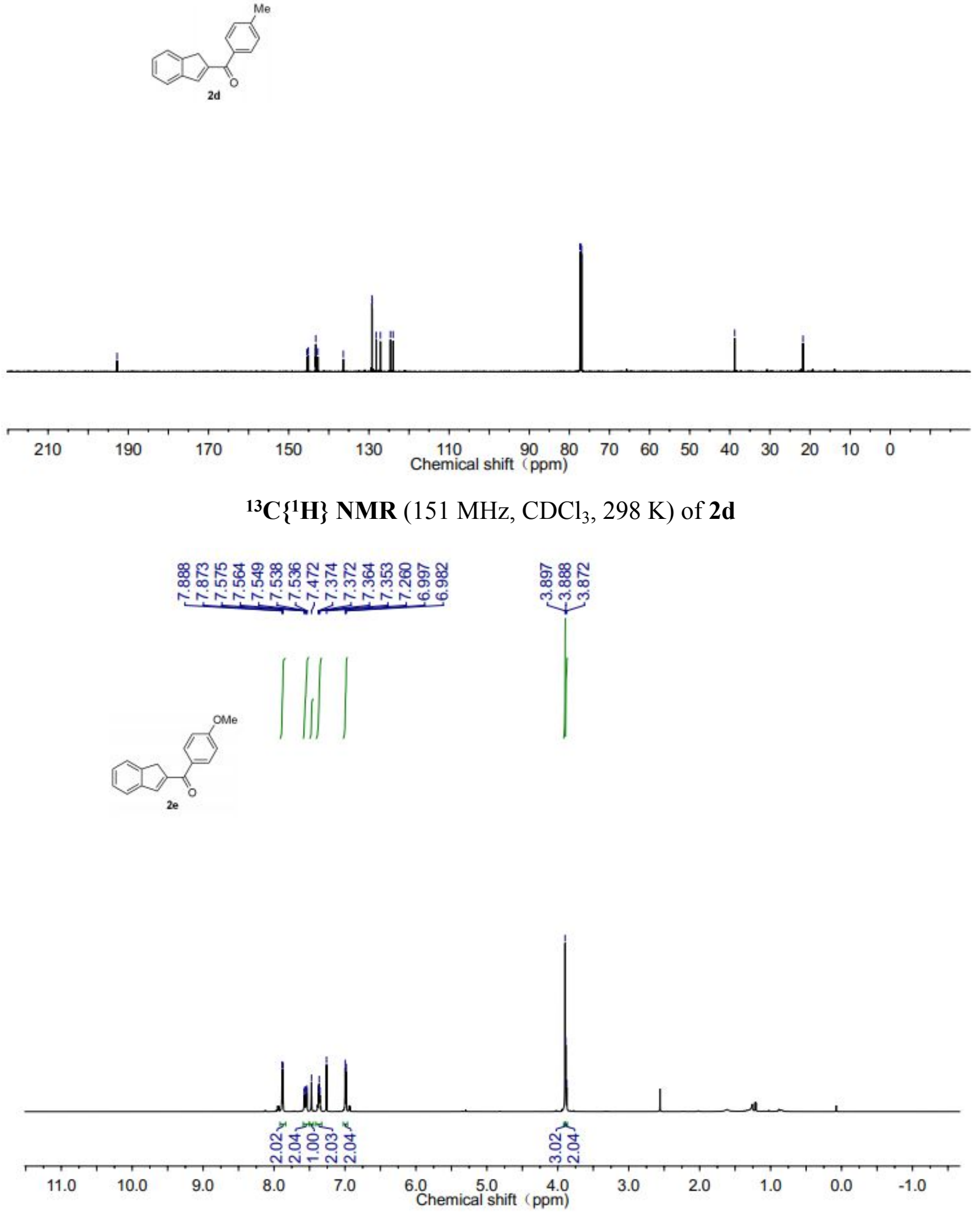
${ }^{1} \mathbf{H}$ NMR $\left(600 \mathrm{MHz}, \mathrm{CDCl}_{3}, 298 \mathrm{~K}\right)$ of $\mathbf{2 e}$

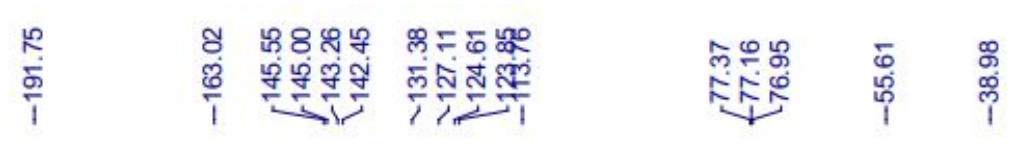

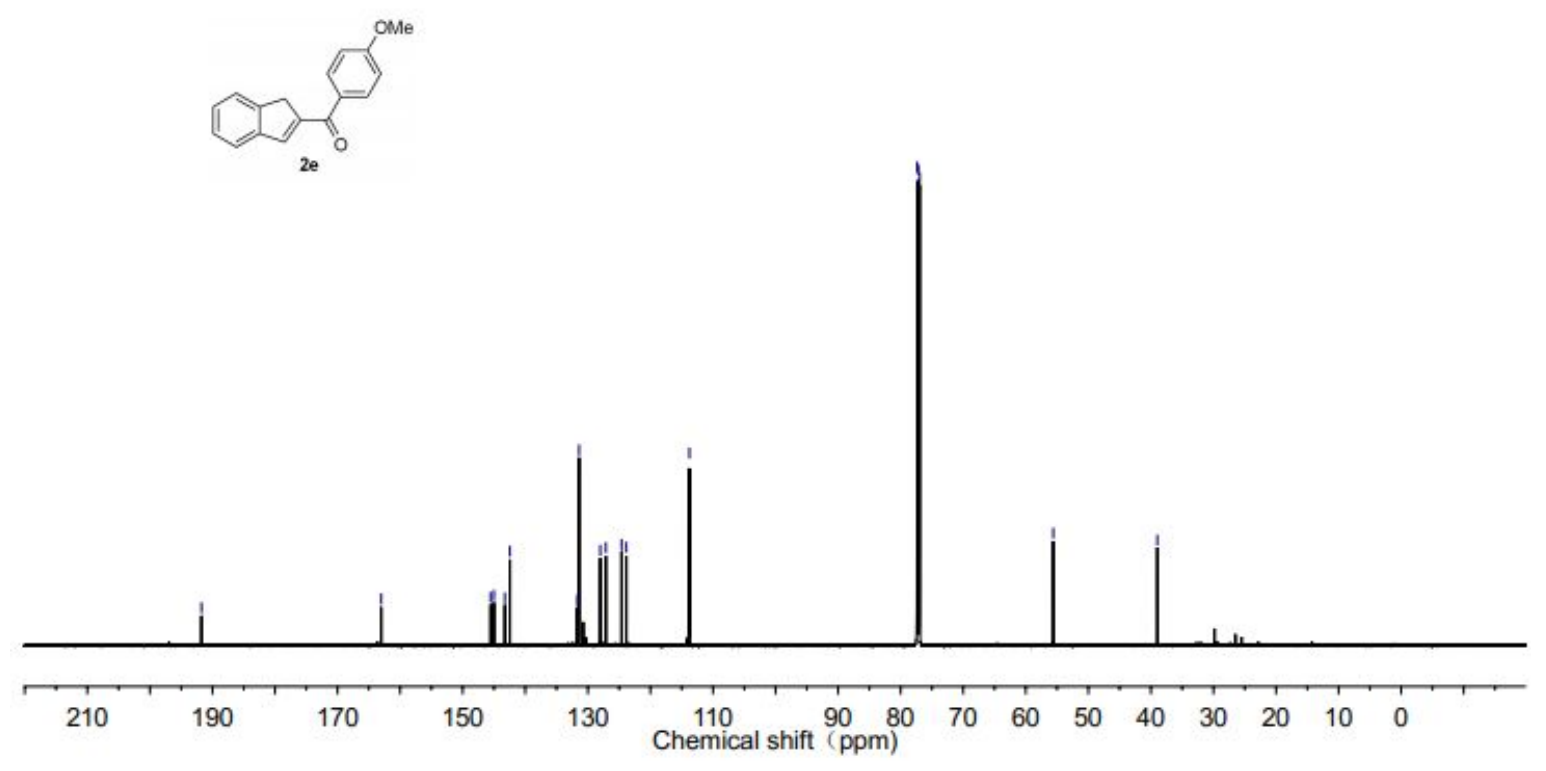

${ }^{13} \mathbf{C}\left\{{ }^{1} \mathbf{H}\right\}$ NMR $\left(151 \mathrm{MHz}, \mathrm{CDCl}_{3}, 298 \mathrm{~K}\right)$ of $\mathbf{2 e}$

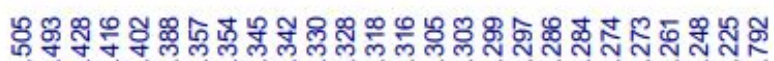

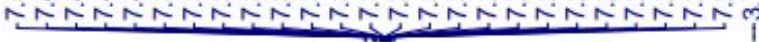
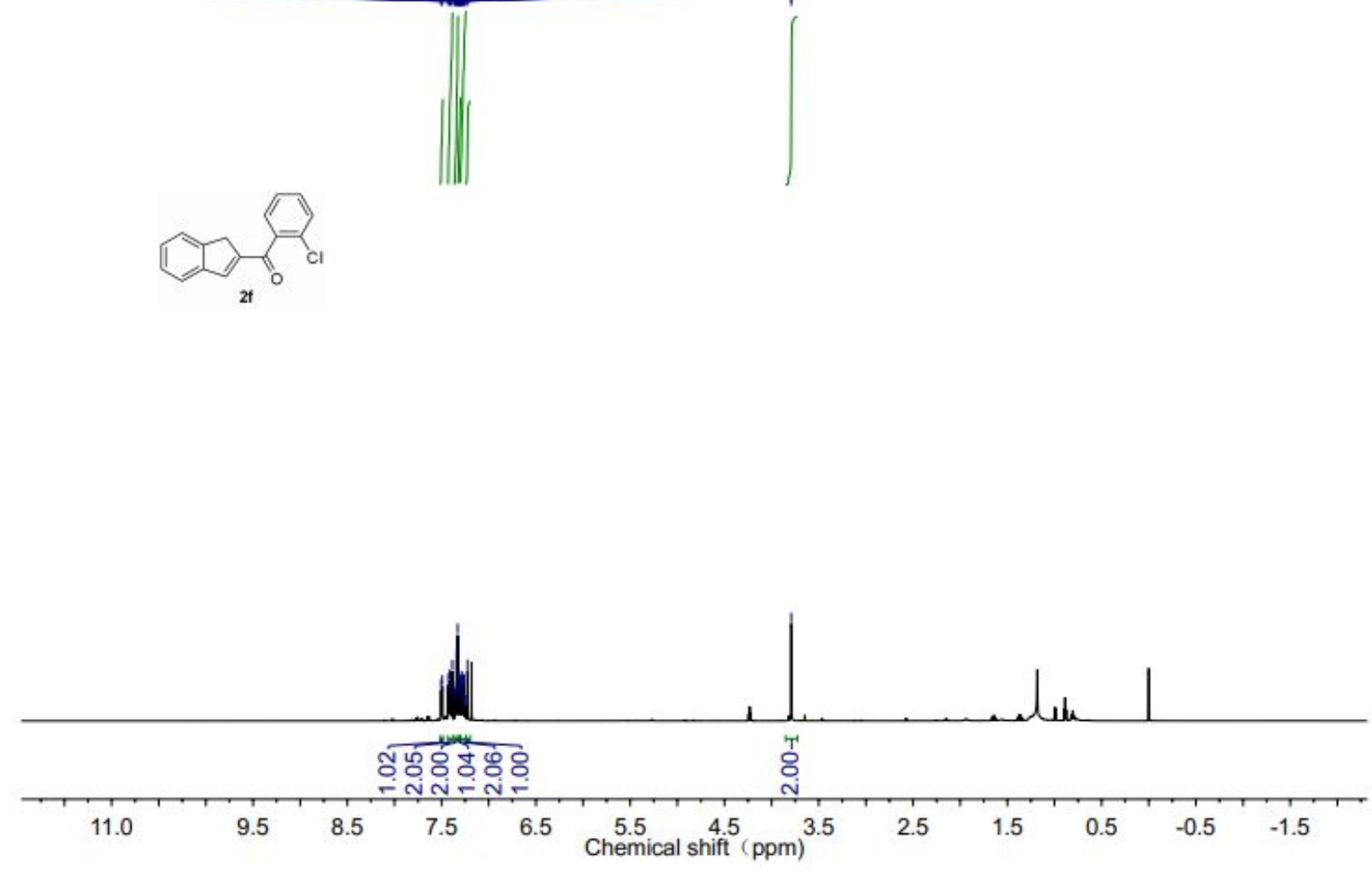
${ }^{1} \mathbf{H}$ NMR $\left(600 \mathrm{MHz}, \mathrm{CDCl}_{3}, 298 \mathrm{~K}\right)$ of $\mathbf{2 f}$
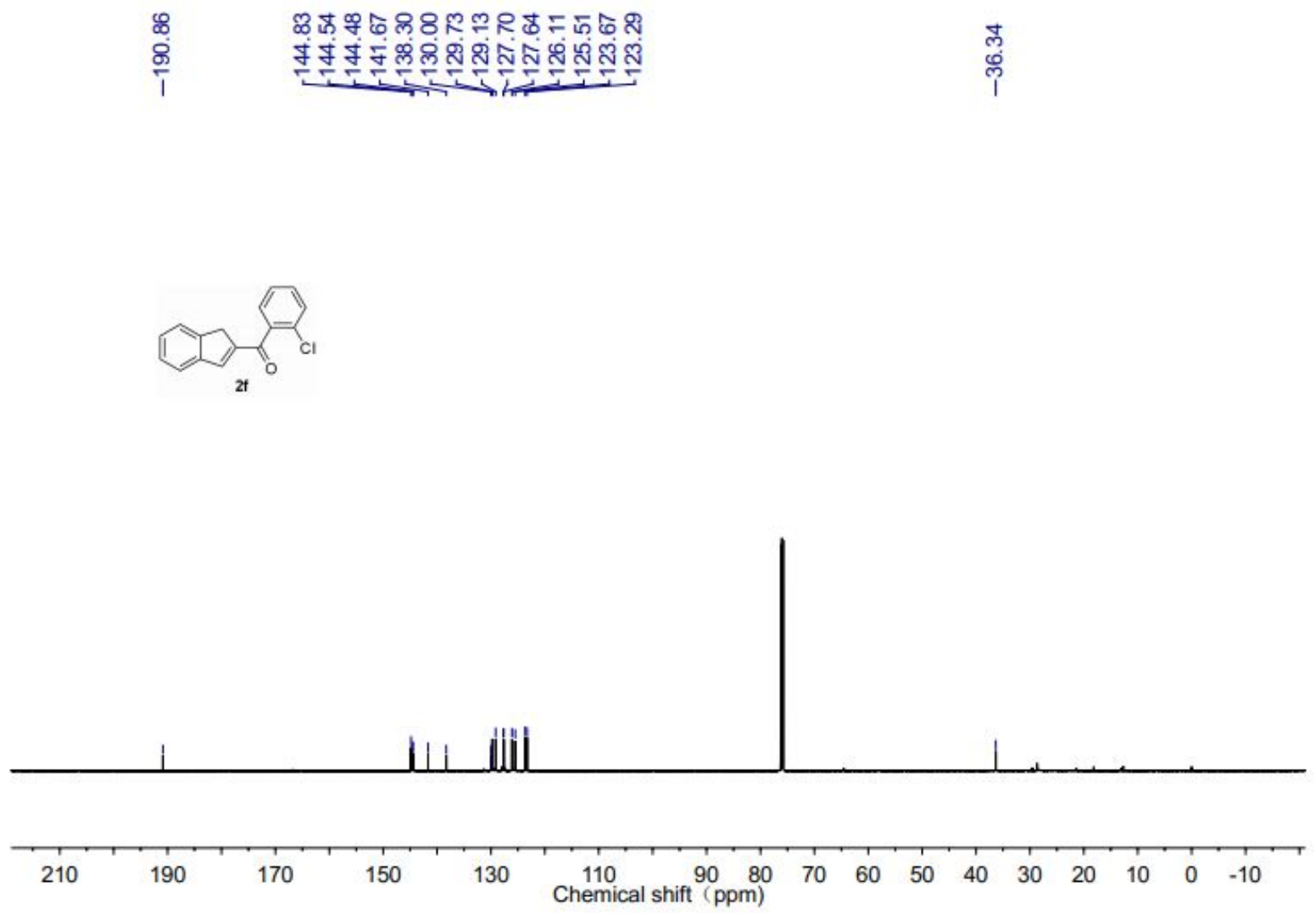

${ }^{13} \mathbf{C}\left\{{ }^{1} \mathbf{H}\right\}$ NMR $\left(151 \mathrm{MHz}, \mathrm{CDCl}_{3}, 298 \mathrm{~K}\right)$ of $\mathbf{2 f}$
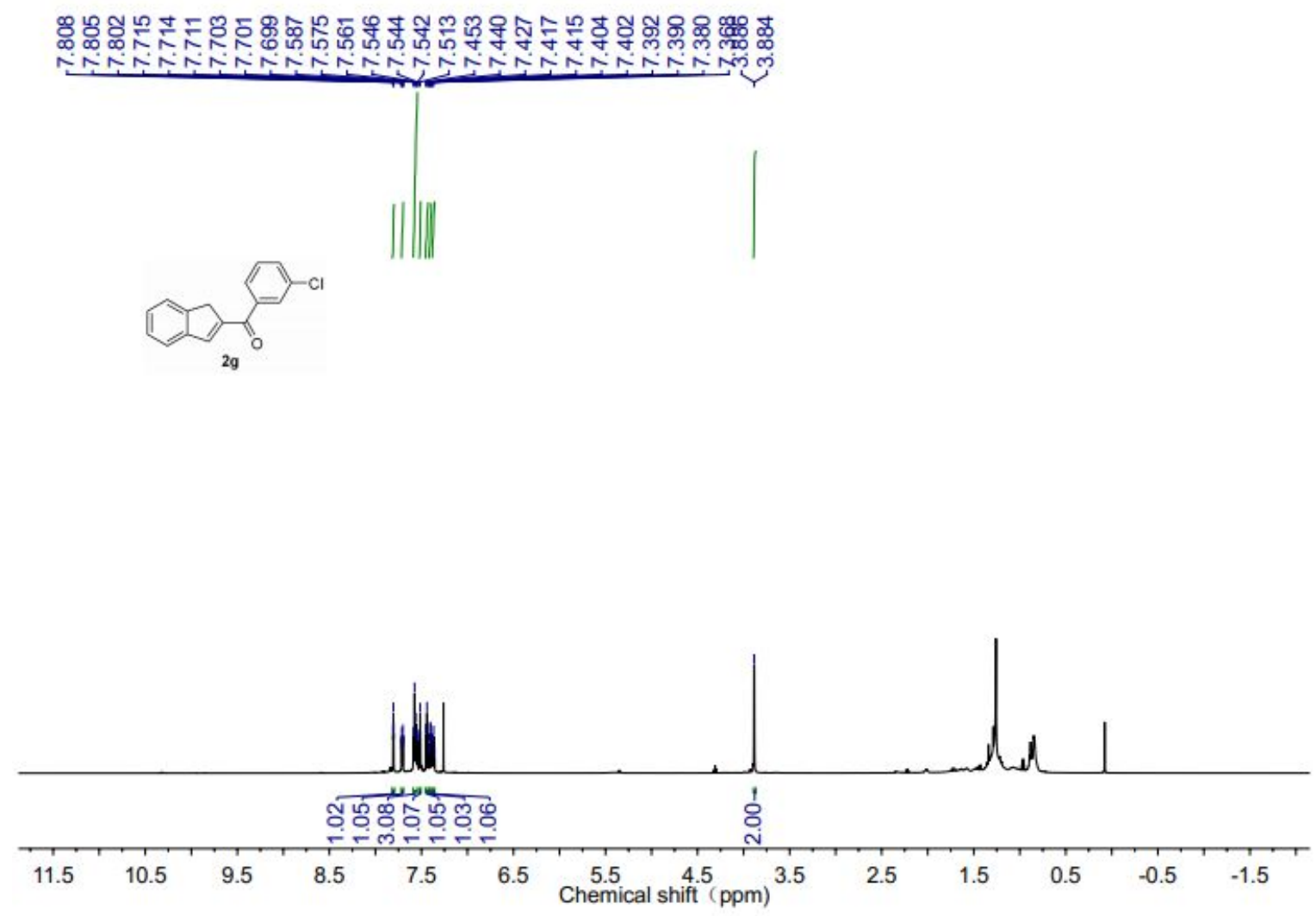
${ }^{\mathbf{1}} \mathbf{H}$ NMR (600 MHz, $\left.\mathrm{CDCl}_{3}, 298 \mathrm{~K}\right)$ of $\mathbf{2 g}$

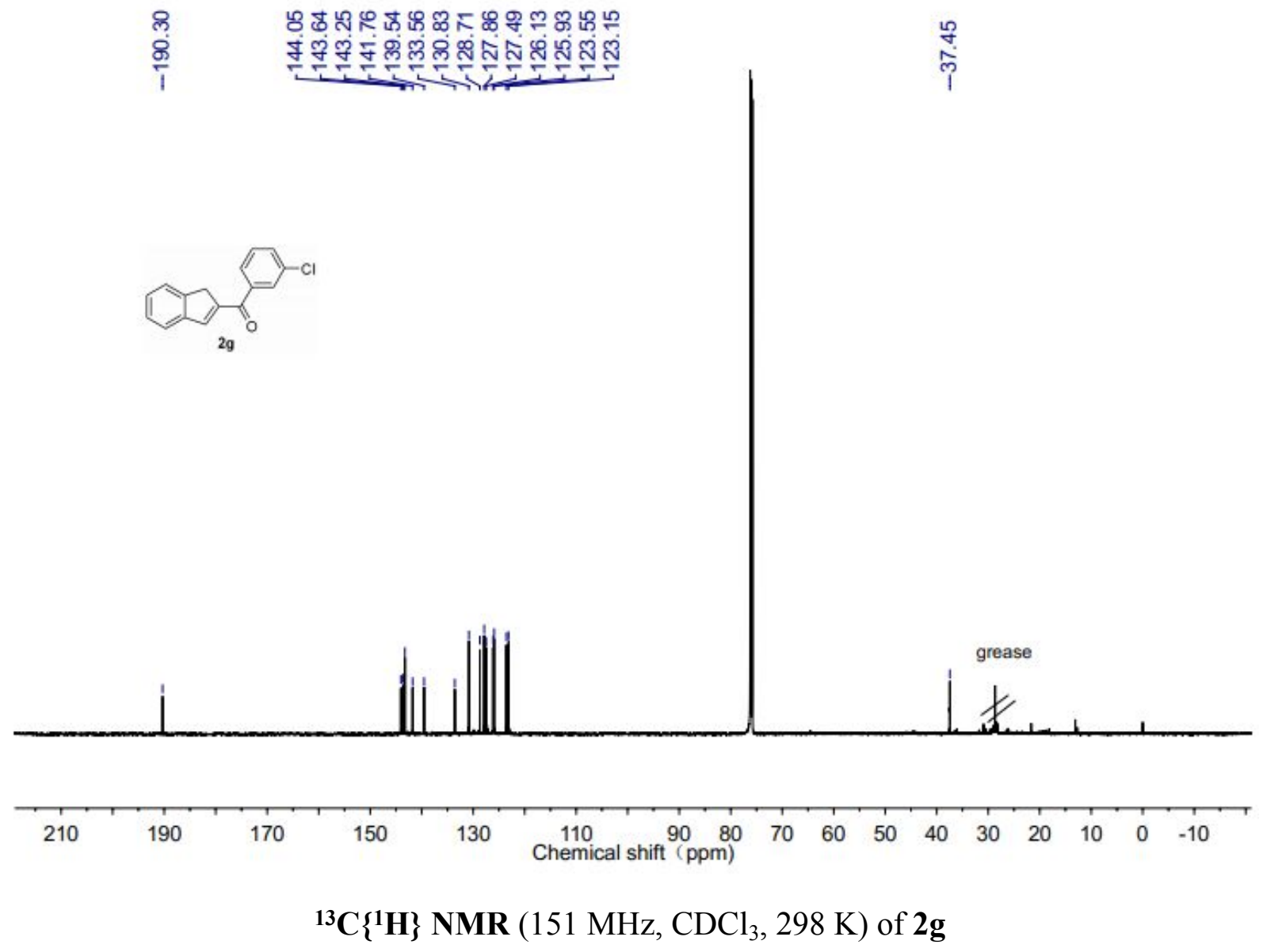



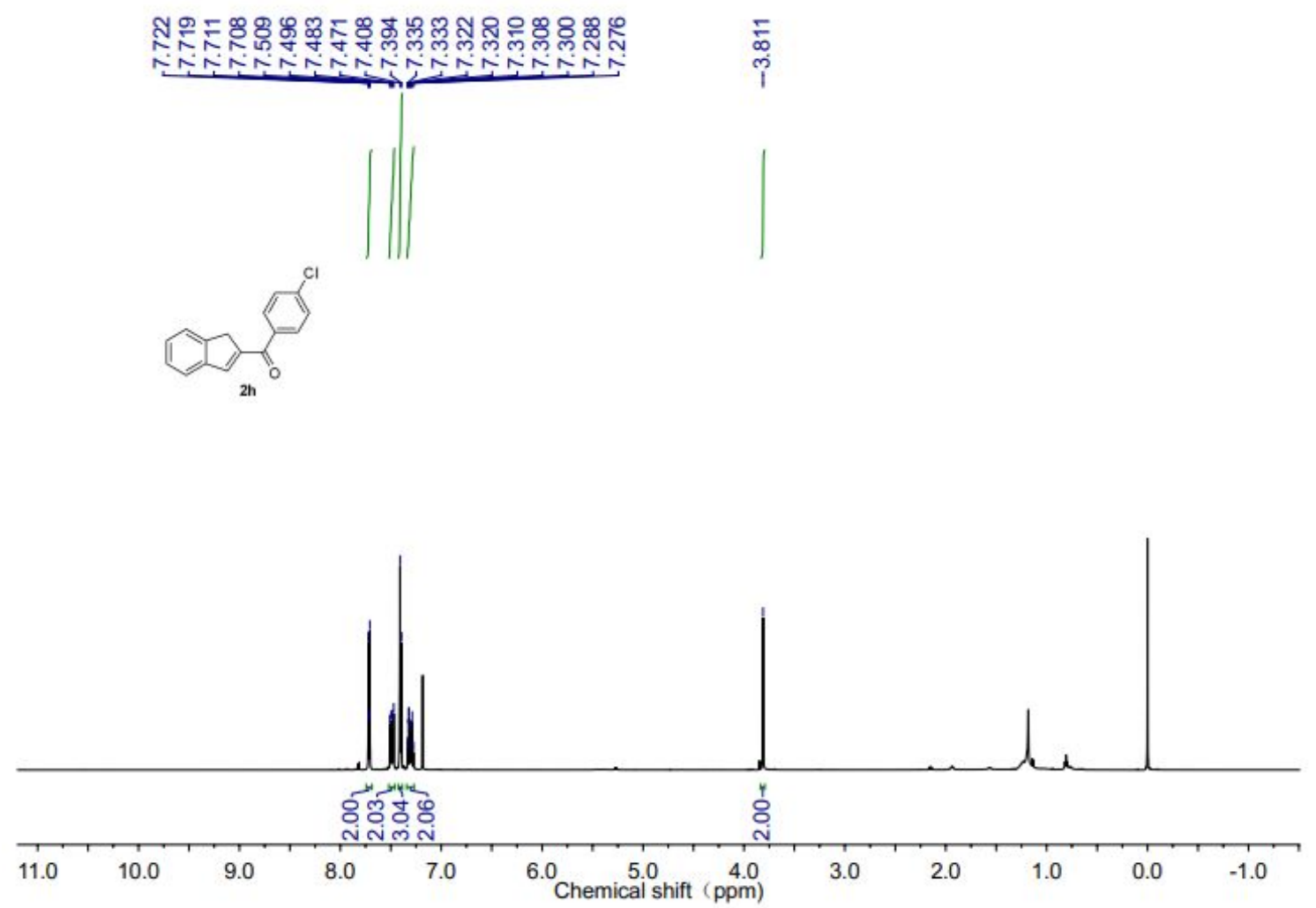

${ }^{1} \mathbf{H}$ NMR $\left(600 \mathrm{MHz}, \mathrm{CDCl}_{3}, 298 \mathrm{~K}\right)$ of $\mathbf{2 h}$
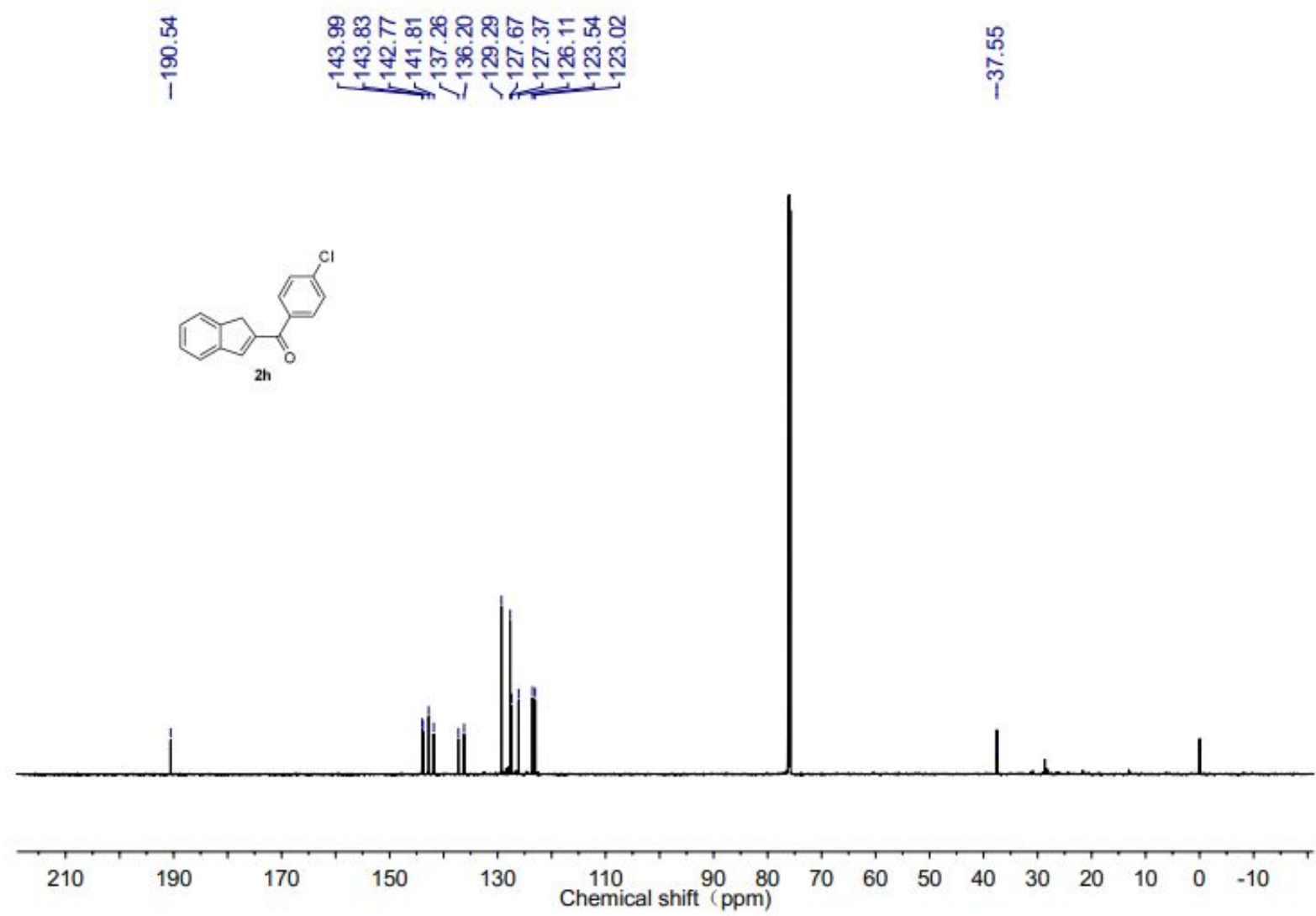

${ }^{13} \mathbf{C}\left\{{ }^{1} \mathbf{H}\right\} \mathbf{N M R}\left(151 \mathrm{MHz}, \mathrm{CDCl}_{3}, 298 \mathrm{~K}\right)$ of $\mathbf{2 h}$ 


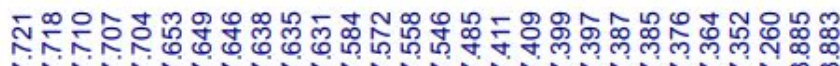

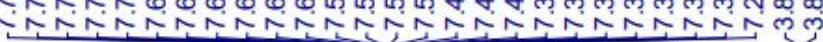
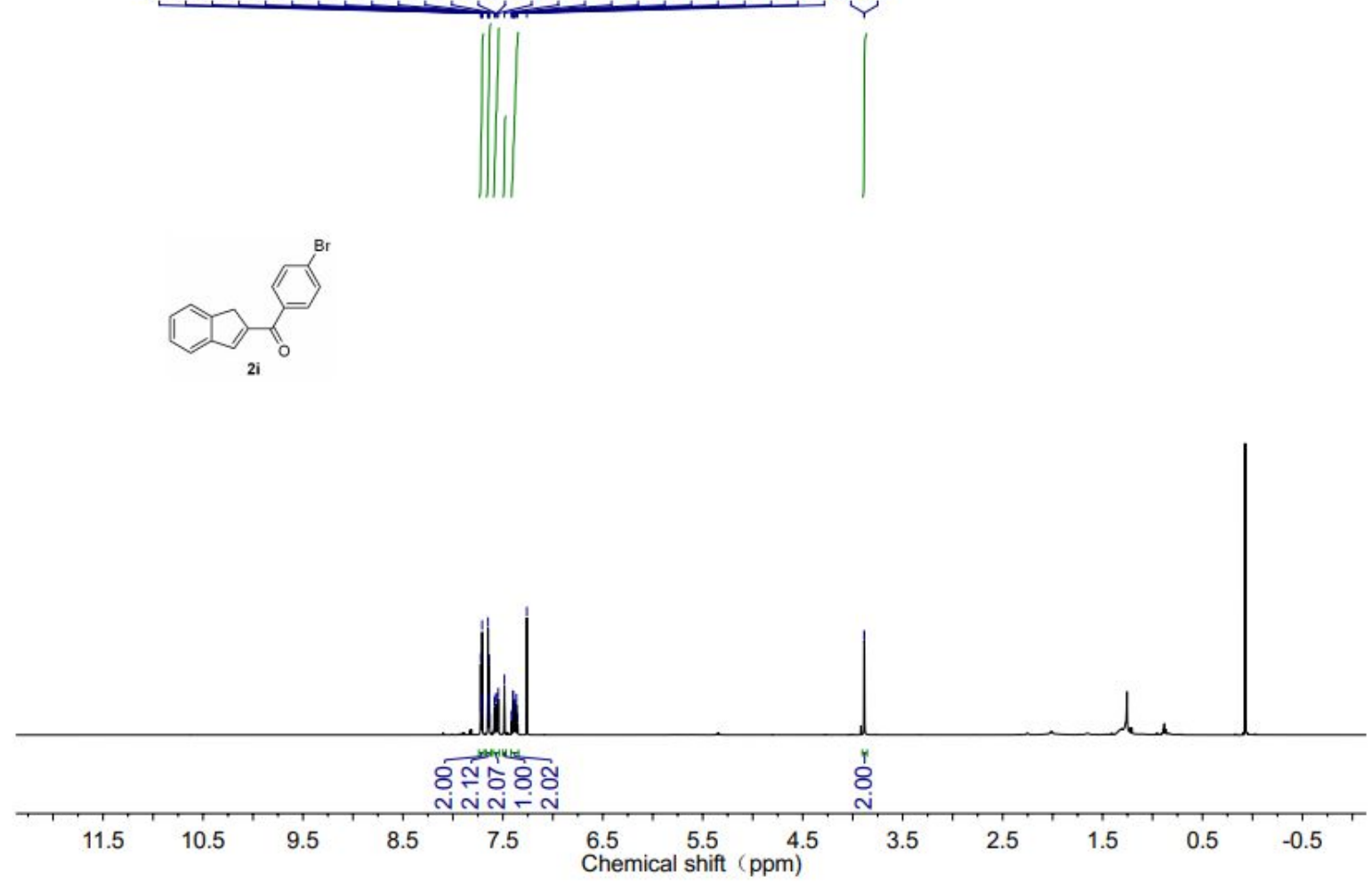

${ }^{1} \mathbf{H}$ NMR $\left(600 \mathrm{MHz}, \mathrm{CDCl}_{3}, 298 \mathrm{~K}\right)$ of $\mathbf{2 i}$
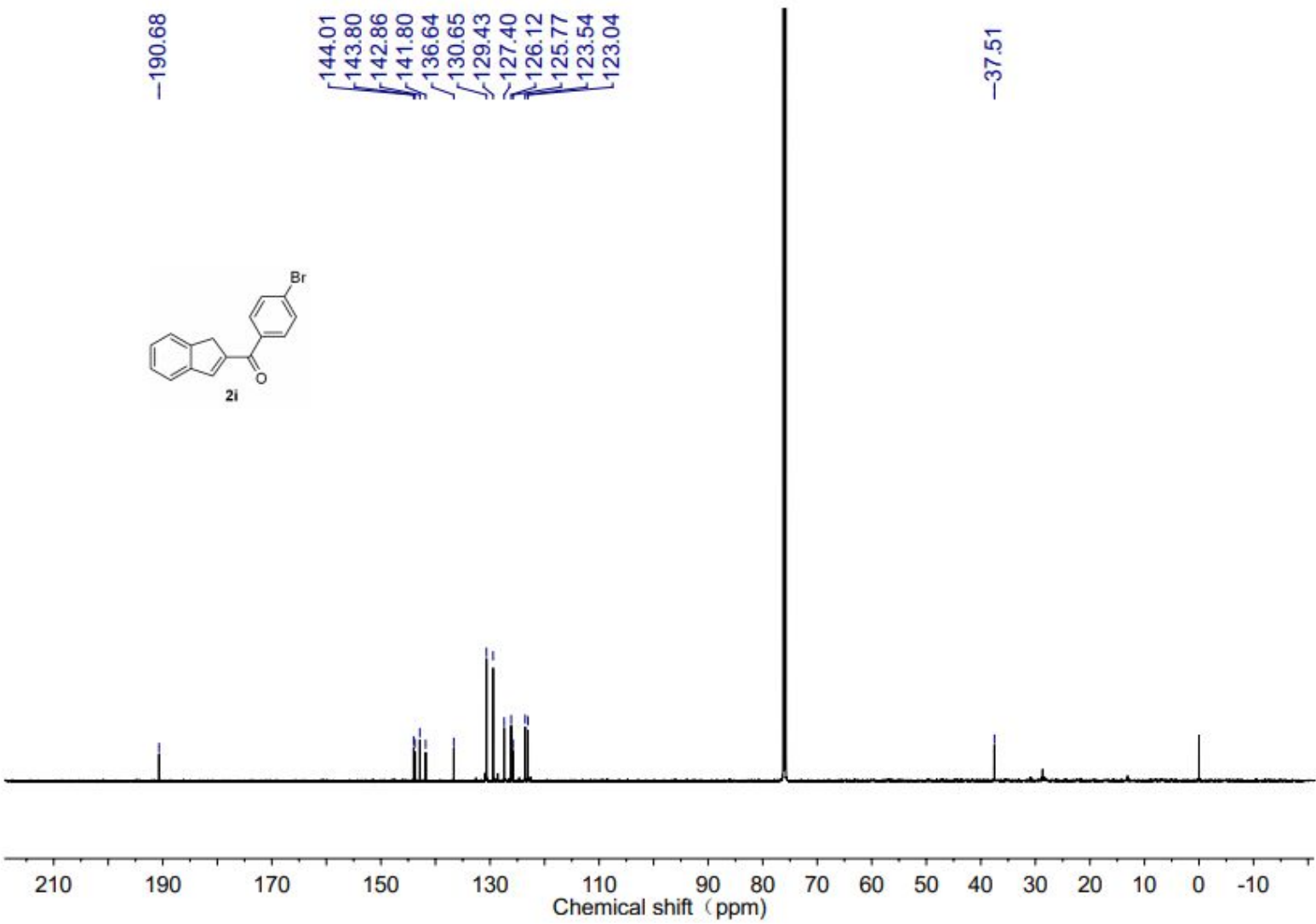

${ }^{13} \mathbf{C}\left\{{ }^{\mathbf{1}} \mathbf{H}\right\} \mathbf{N M R}\left(151 \mathrm{MHz}, \mathrm{CDCl}_{3}, 298 \mathrm{~K}\right)$ of $\mathbf{2 i}$ 


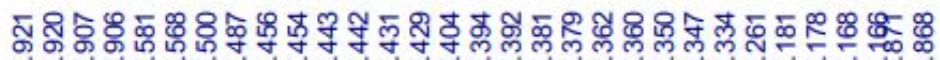

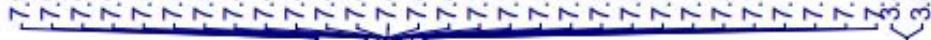
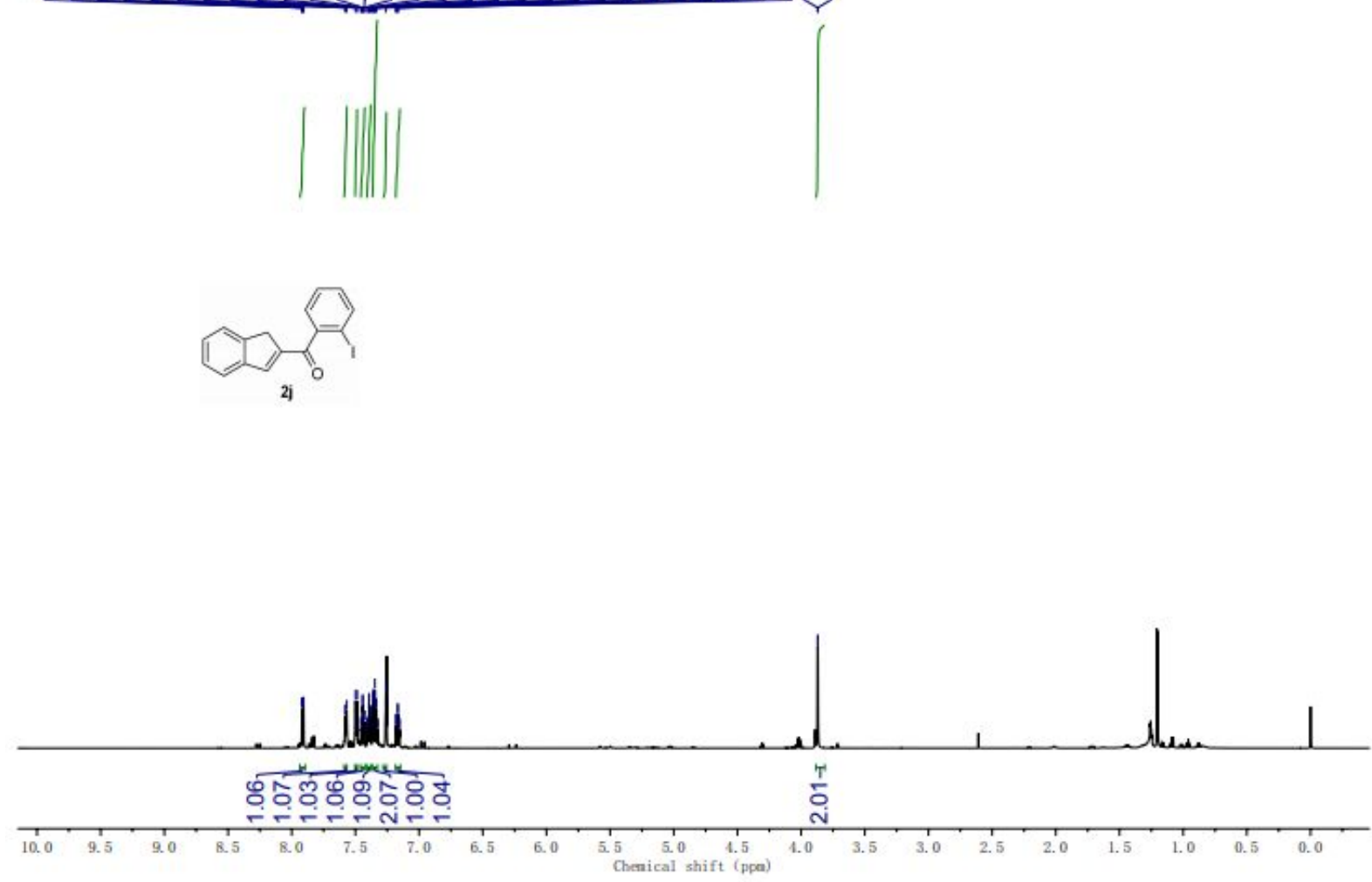

${ }^{\mathbf{1}} \mathbf{H}$ NMR $\left(600 \mathrm{MHz}, \mathrm{CDCl}_{3}, 298 \mathrm{~K}\right)$ of $\mathbf{2} \mathbf{j}$
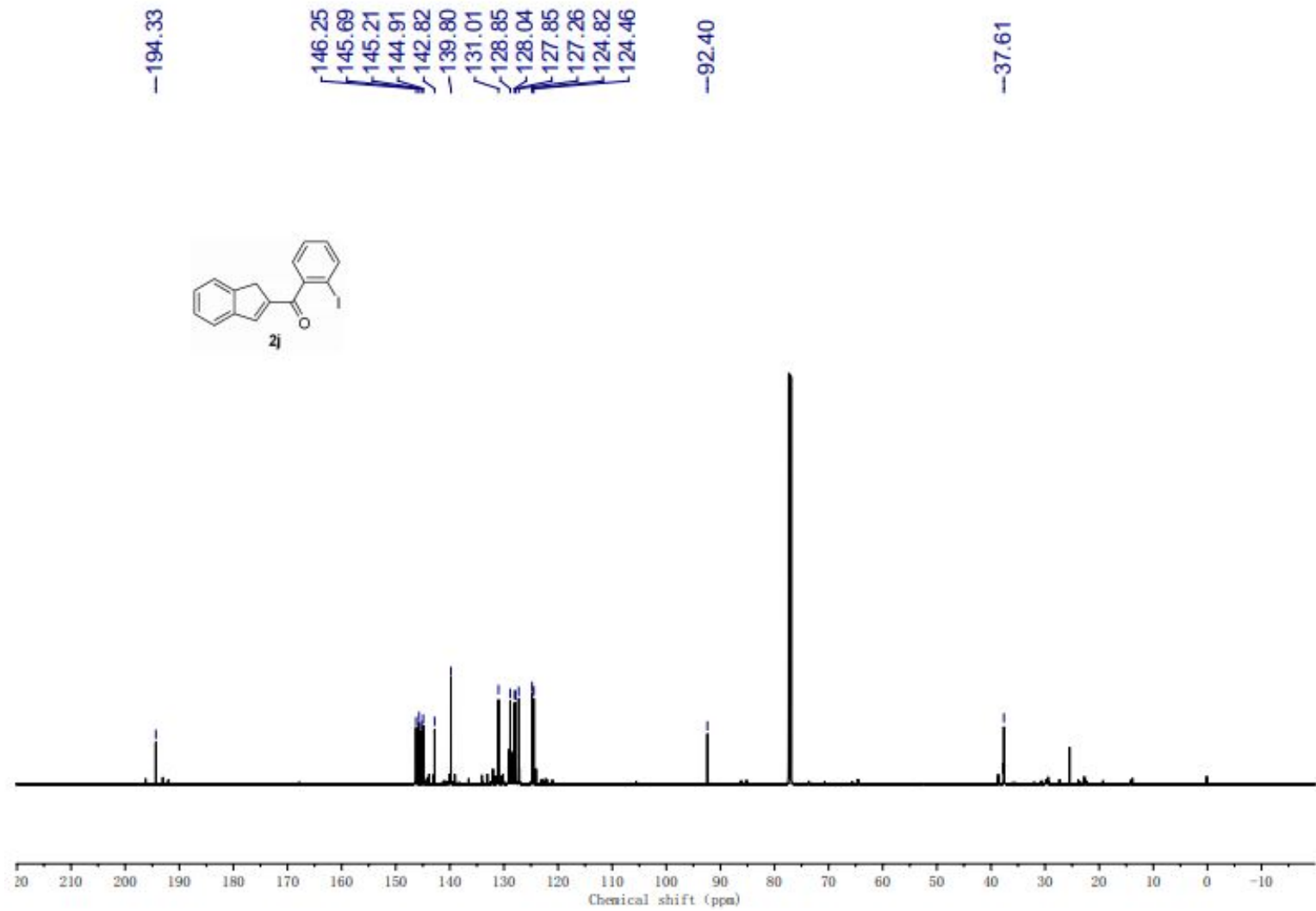

${ }^{13} \mathbf{C}\left\{{ }^{\mathbf{1}} \mathbf{H}\right\} \mathbf{N M R}\left(151 \mathrm{MHz}, \mathrm{CDCl}_{3}, 298 \mathrm{~K}\right)$ of $\mathbf{2} \mathbf{j}$ 

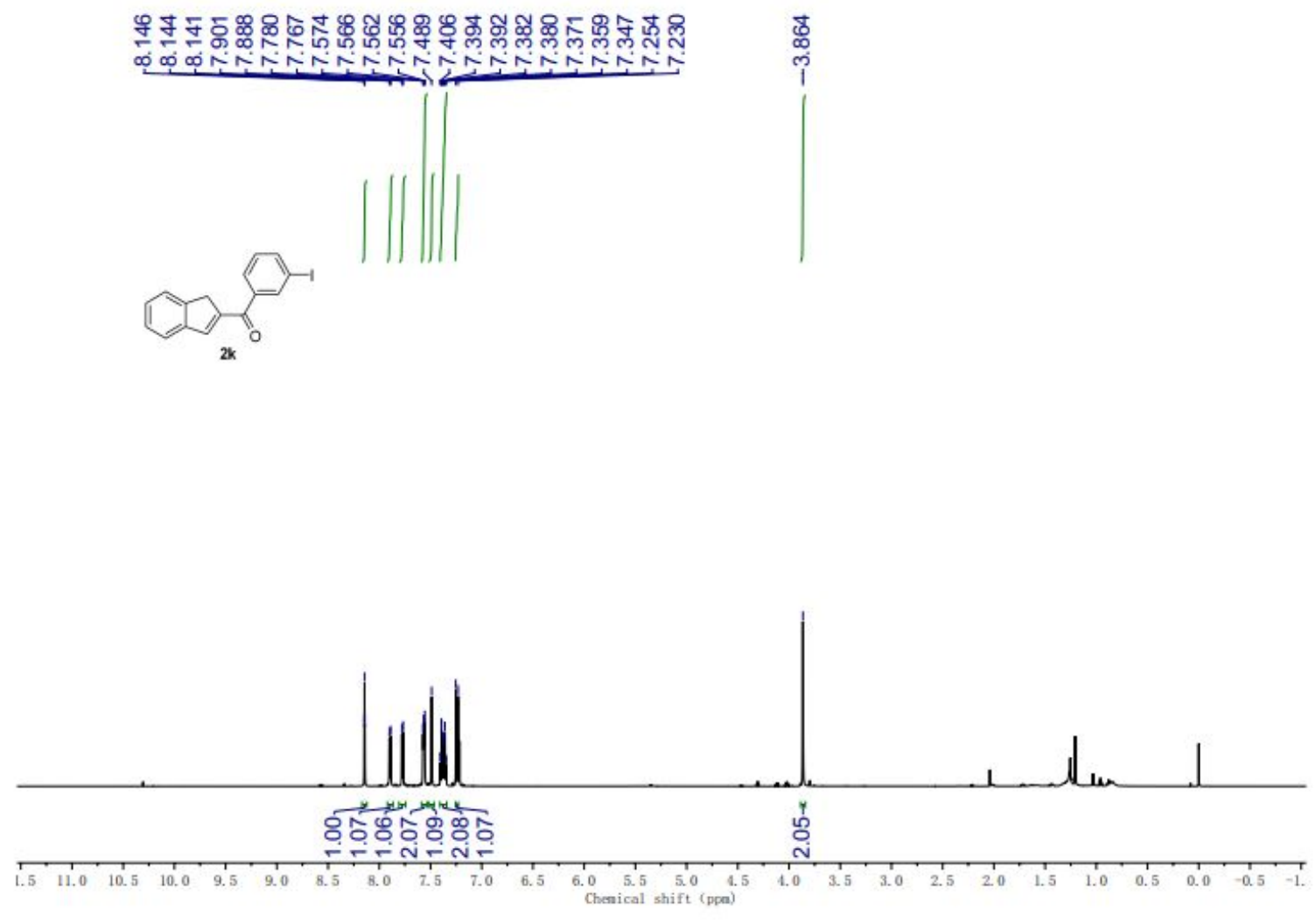

${ }^{1} \mathbf{H}$ NMR $\left(600 \mathrm{MHz}, \mathrm{CDCl}_{3}, 298 \mathrm{~K}\right)$ of $\mathbf{2 k}$
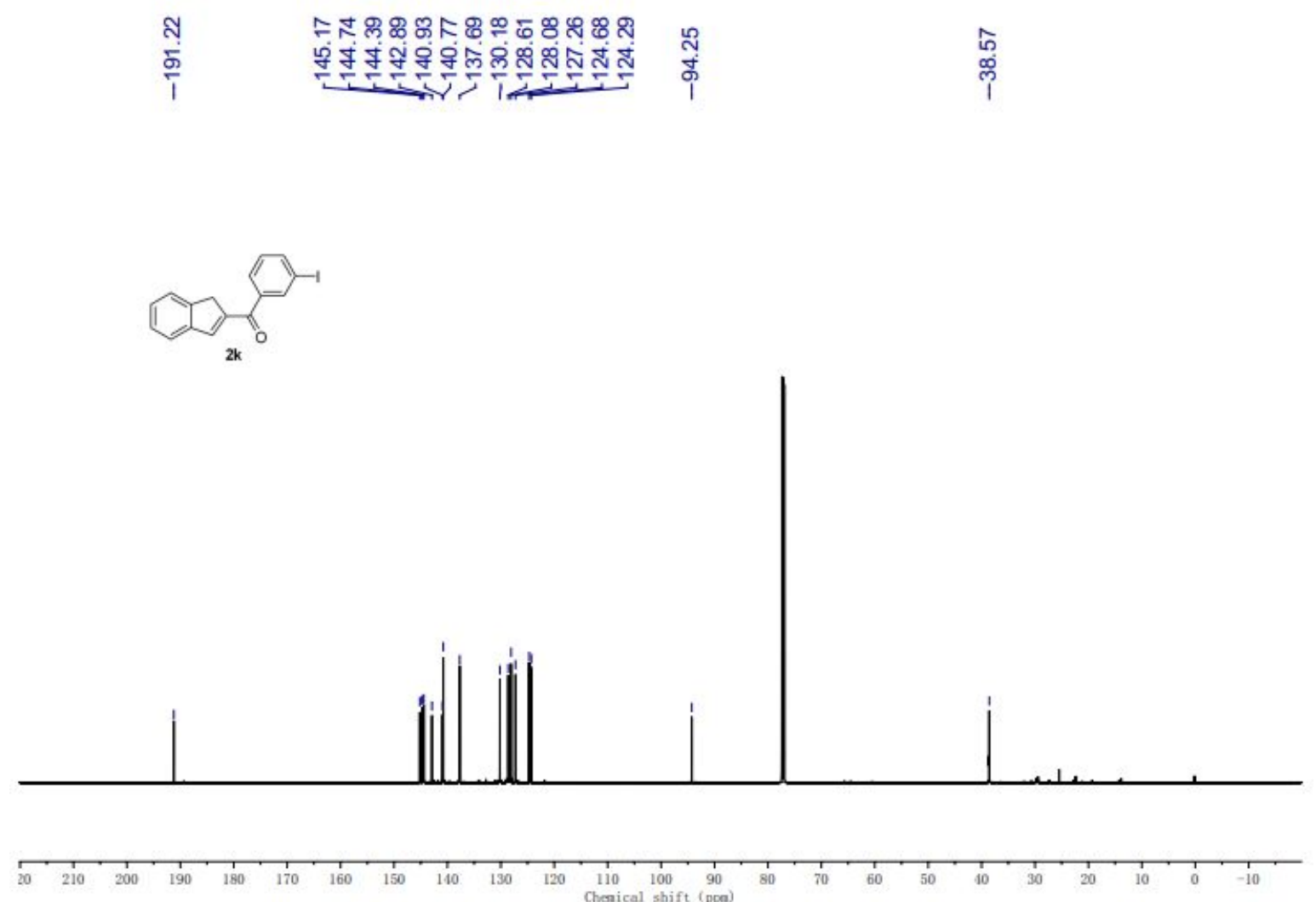

${ }^{13} \mathbf{C}\left\{{ }^{1} \mathbf{H}\right\}$ NMR $\left(151 \mathrm{MHz}, \mathrm{CDCl}_{3}, 298 \mathrm{~K}\right)$ of $\mathbf{2 k}$ 

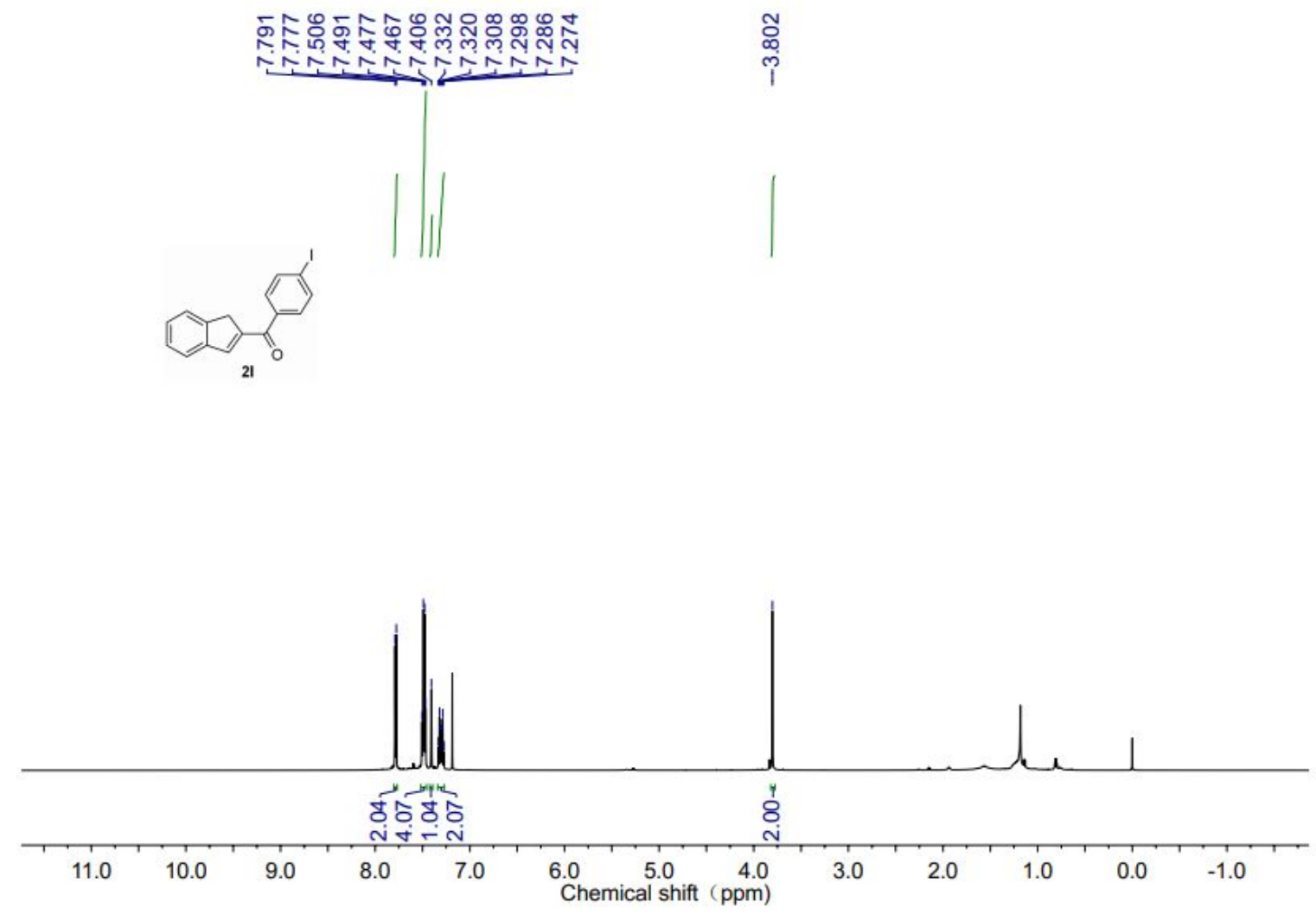

${ }^{1} \mathbf{H}$ NMR $\left(600 \mathrm{MHz}, \mathrm{CDCl}_{3}, 298 \mathrm{~K}\right)$ of $\mathbf{2 l}$

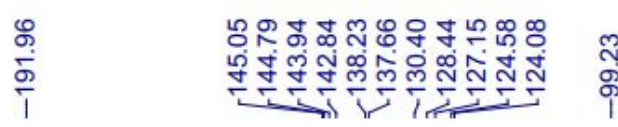

సิ

$\bar{n}$
0
0
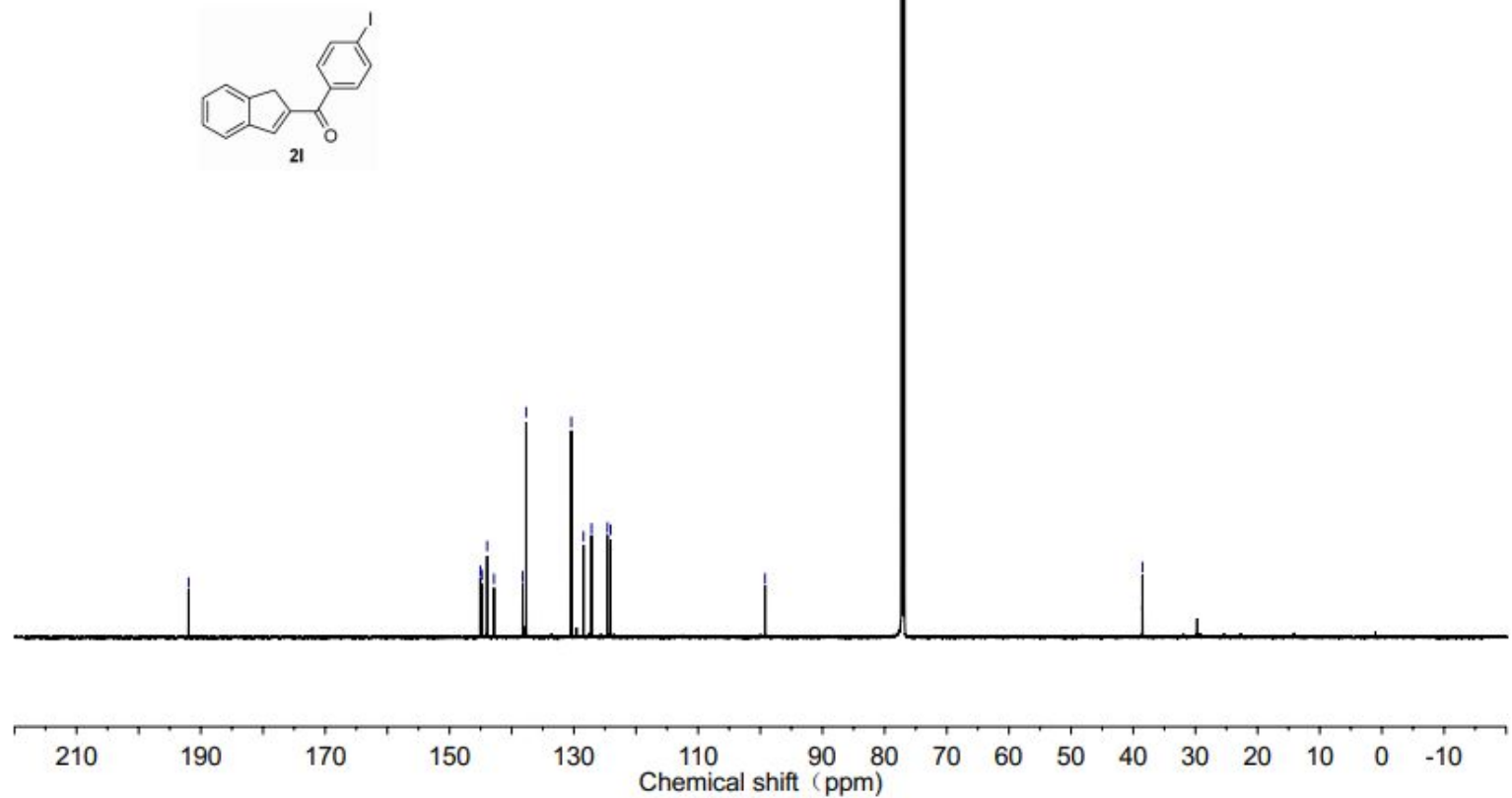

${ }^{13} \mathbf{C}\left\{{ }^{\mathbf{1}} \mathbf{H}\right\} \mathbf{N M R}\left(151 \mathrm{MHz}, \mathrm{CDCl}_{3}, 298 \mathrm{~K}\right)$ of $\mathbf{2 l}$ 

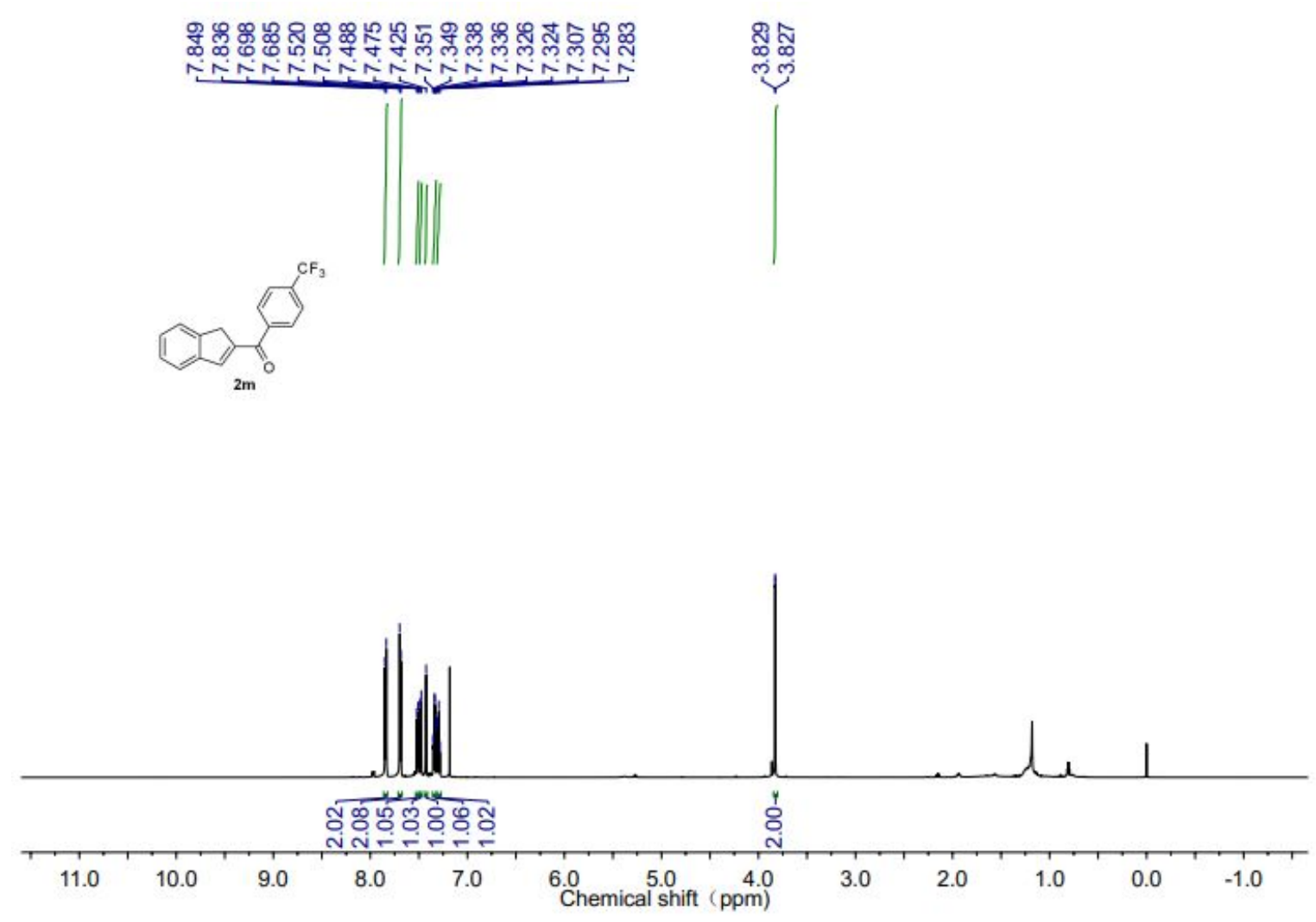

${ }^{1} \mathbf{H}$ NMR $\left(600 \mathrm{MHz}, \mathrm{CDCl}_{3}, 298 \mathrm{~K}\right)$ of $\mathbf{2 m}$

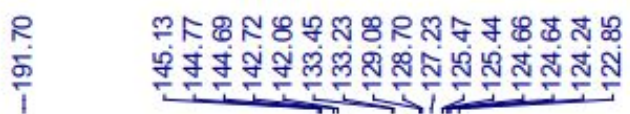
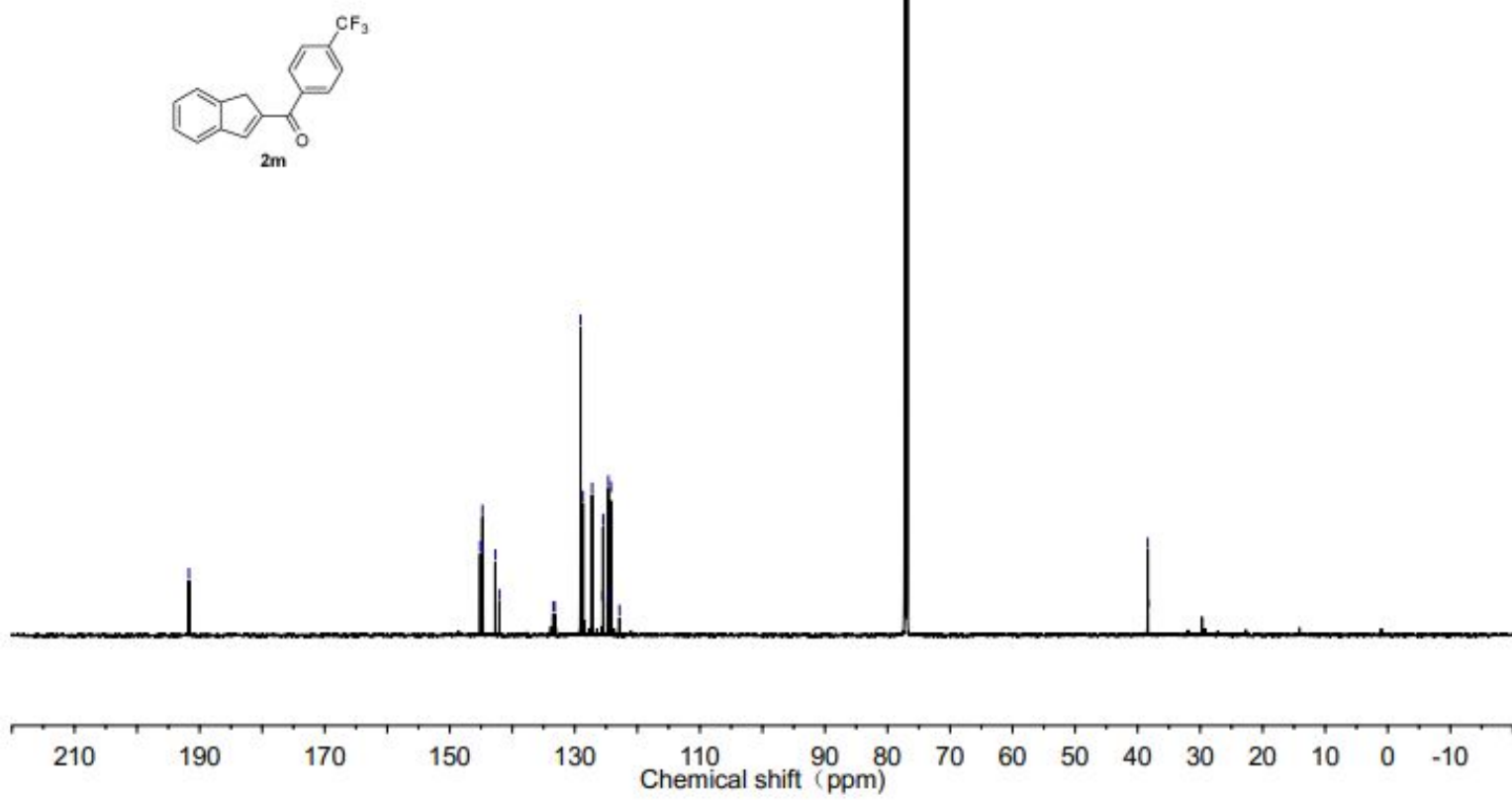

${ }^{13} \mathbf{C}\left\{{ }^{1} \mathbf{H}\right\}$ NMR $\left(151 \mathrm{MHz}, \mathrm{CDCl}_{3}, 298 \mathrm{~K}\right)$ of $\mathbf{2 m}$ 

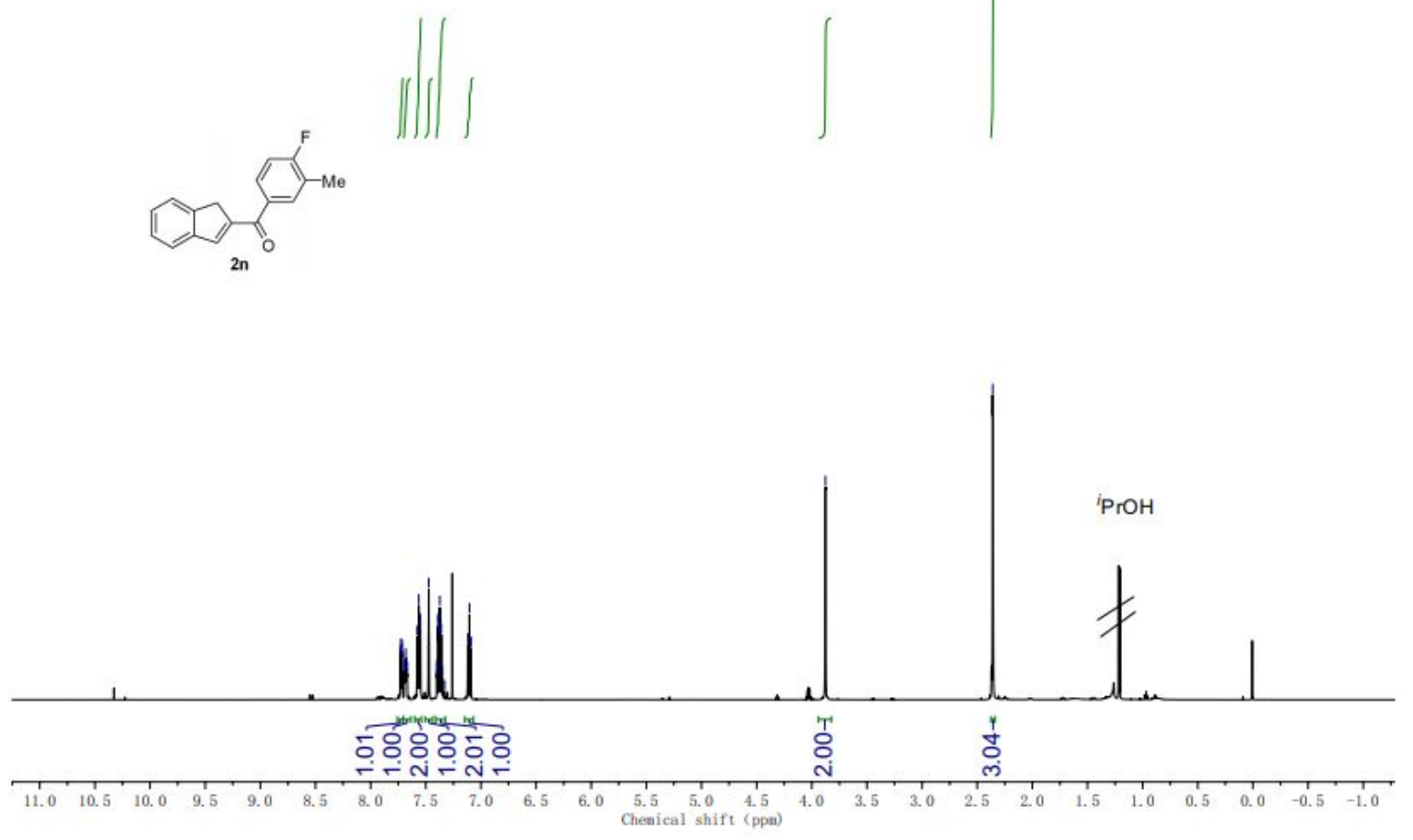

${ }^{1} \mathbf{H}$ NMR $\left(600 \mathrm{MHz}, \mathrm{CDCl}_{3}, 298 \mathrm{~K}\right)$ of $\mathbf{2 n}$

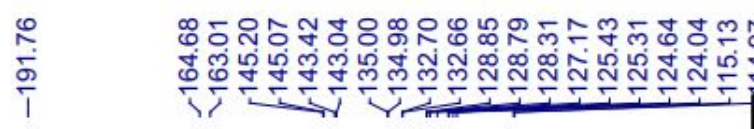

$\begin{array}{ll}\infty & N \\ \infty & \stackrel{N}{+}\end{array}$
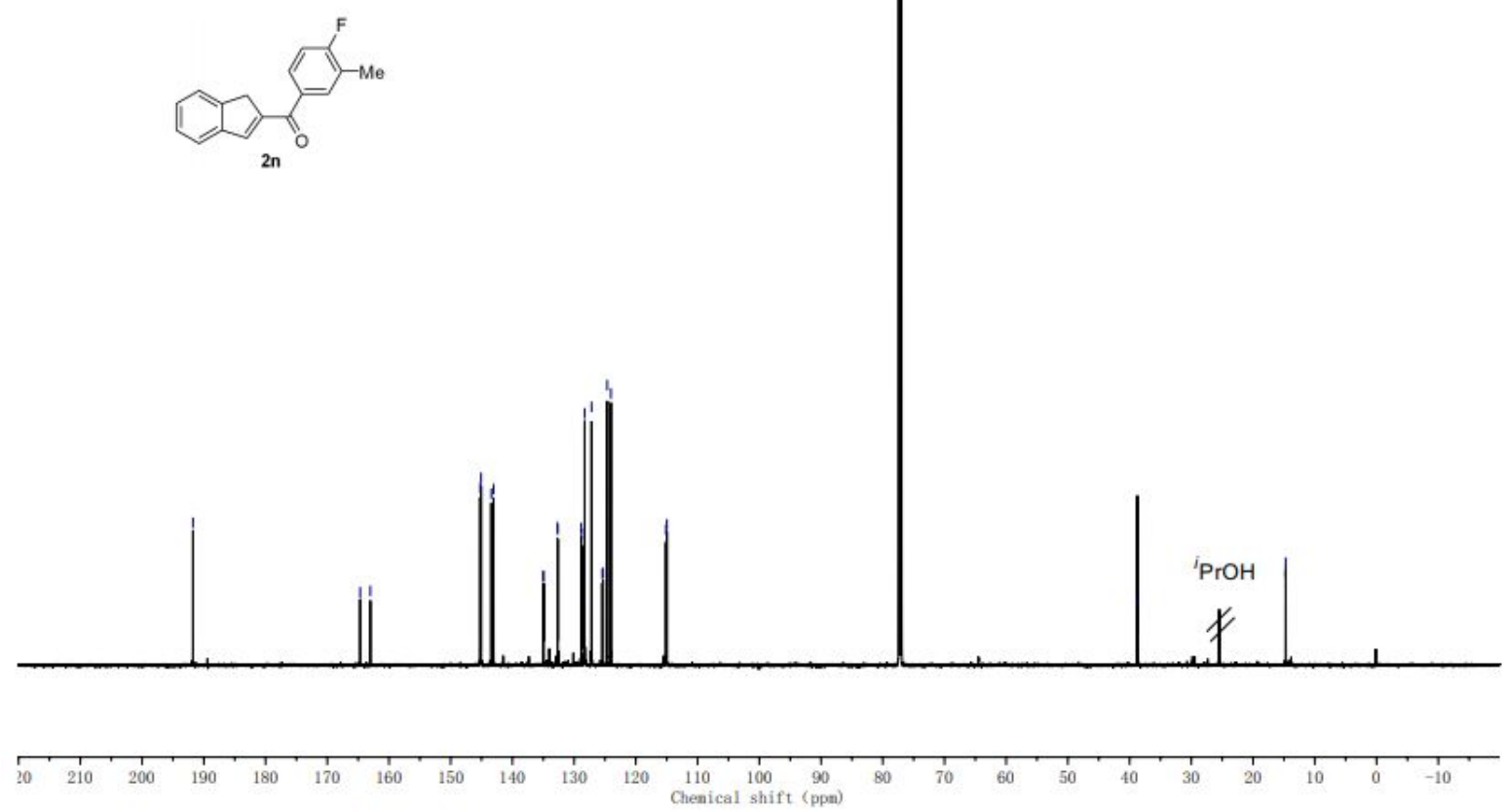

${ }^{13} \mathbf{C}\left\{{ }^{1} \mathbf{H}\right\} \mathbf{N M R}\left(151 \mathrm{MHz}, \mathrm{CDCl}_{3}, 298 \mathrm{~K}\right)$ of $\mathbf{2 n}$ 

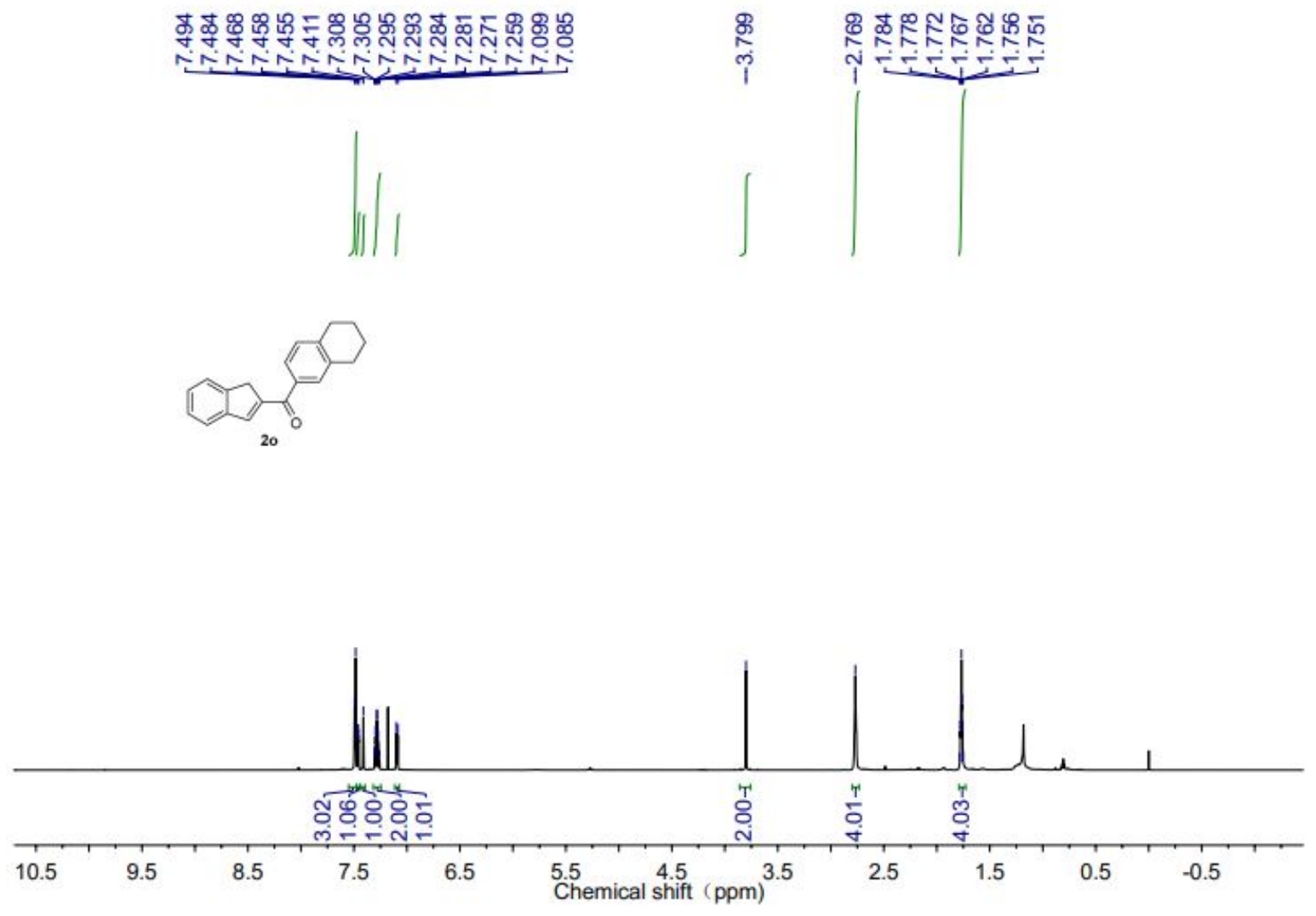

${ }^{1} \mathbf{H}$ NMR (600 MHz, $\left.\mathrm{CDCl}_{3}, 298 \mathrm{~K}\right)$ of $\mathbf{2 0}$

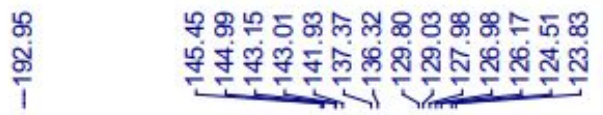

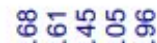

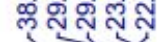
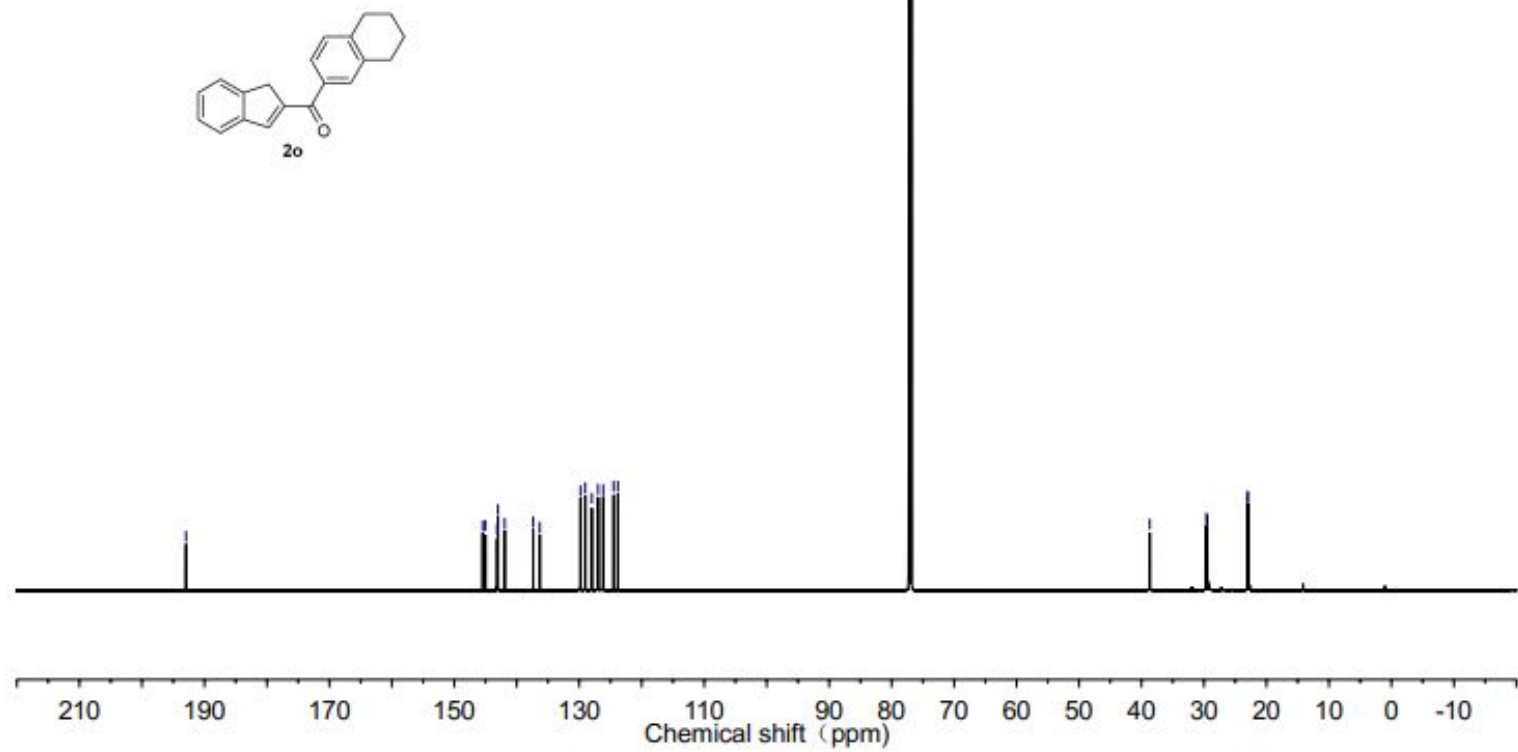

${ }^{13} \mathbf{C}\left\{{ }^{1} \mathbf{H}\right\} \mathbf{N M R}\left(151 \mathrm{MHz}, \mathrm{CDCl}_{3}, 298 \mathrm{~K}\right)$ of $\mathbf{2 0}$ 

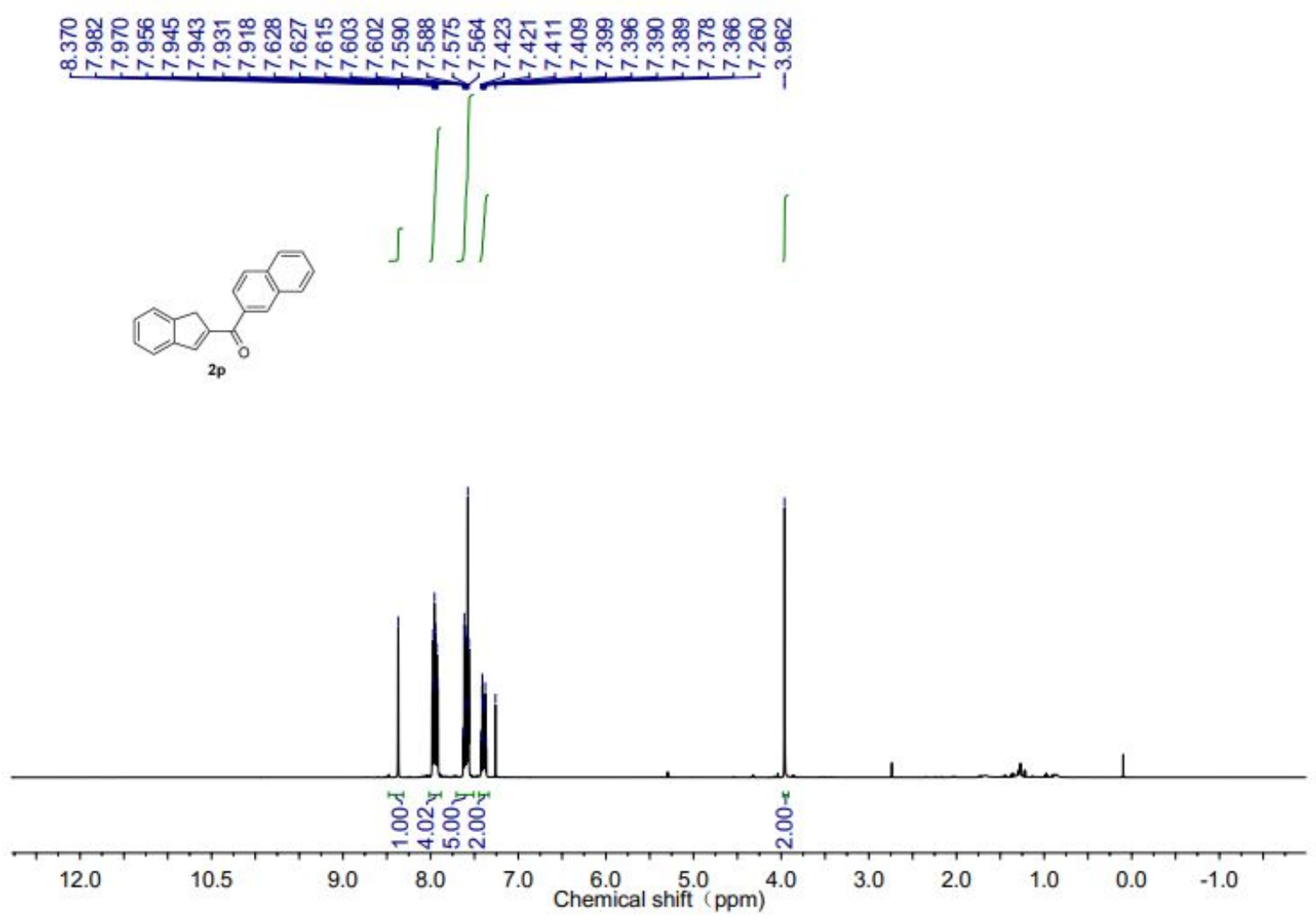

${ }^{1} \mathbf{H}$ NMR $\left(600 \mathrm{MHz}, \mathrm{CDCl}_{3}, 298 \mathrm{~K}\right)$ of $\mathbf{2 p}$
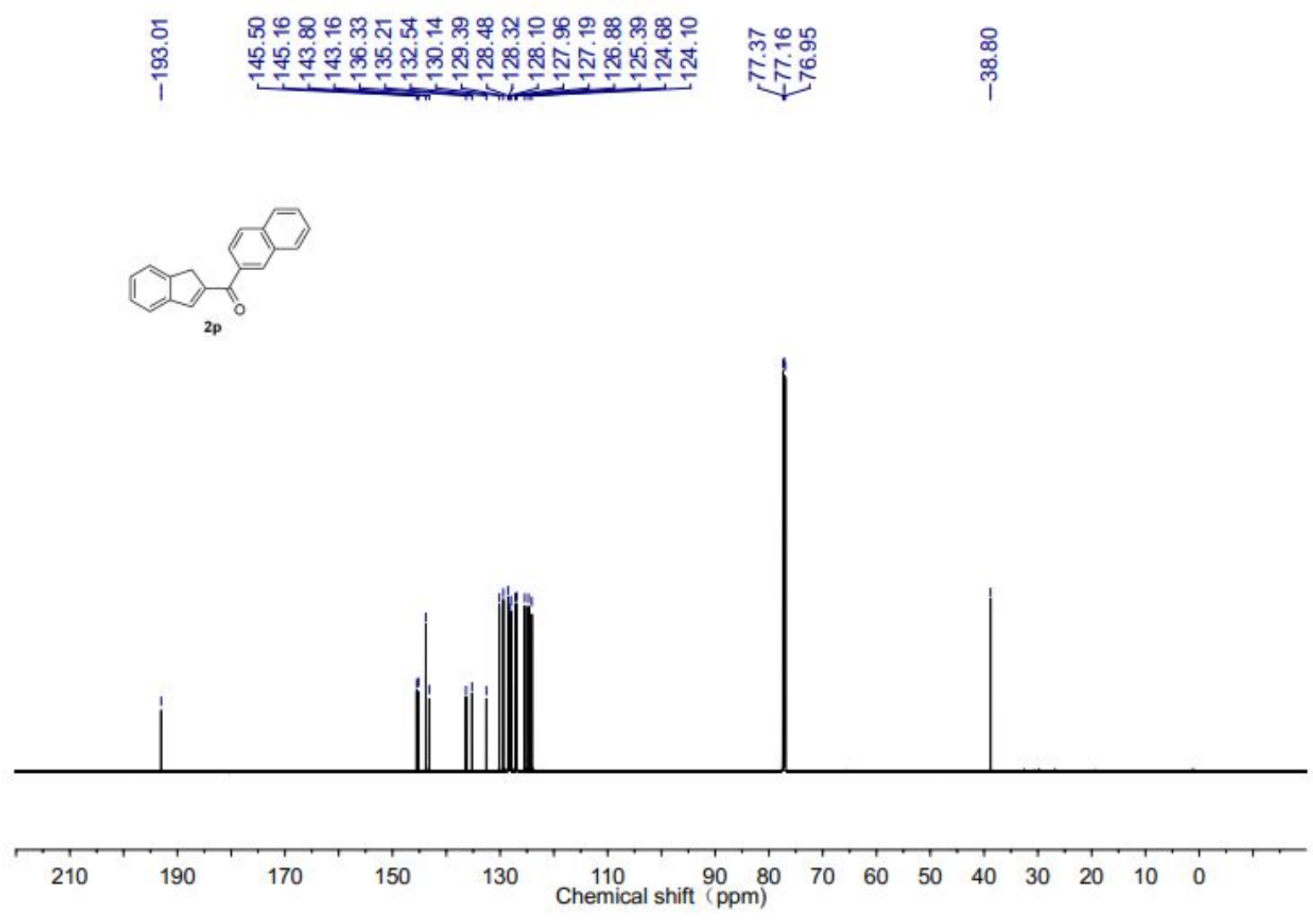

${ }^{13} \mathbf{C}\left\{{ }^{1} \mathbf{H}\right\} \mathbf{N M R}\left(151 \mathrm{MHz}, \mathrm{CDCl}_{3}, 298 \mathrm{~K}\right)$ of $\mathbf{2 p}$ 


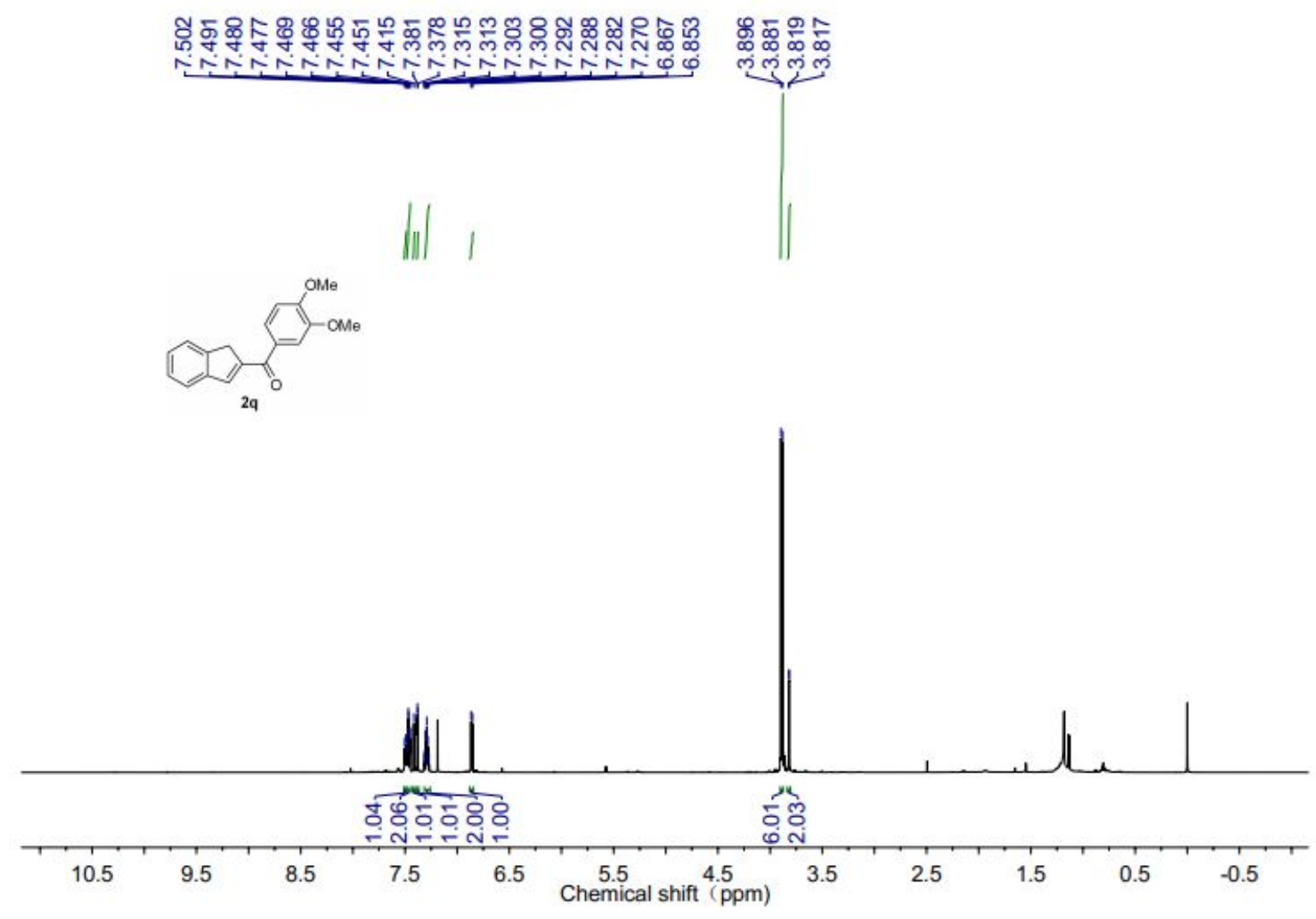

${ }^{\mathbf{1}} \mathbf{H}$ NMR (600 MHz, $\left.\mathrm{CDCl}_{3}, 298 \mathrm{~K}\right)$ of $\mathbf{2 q}$
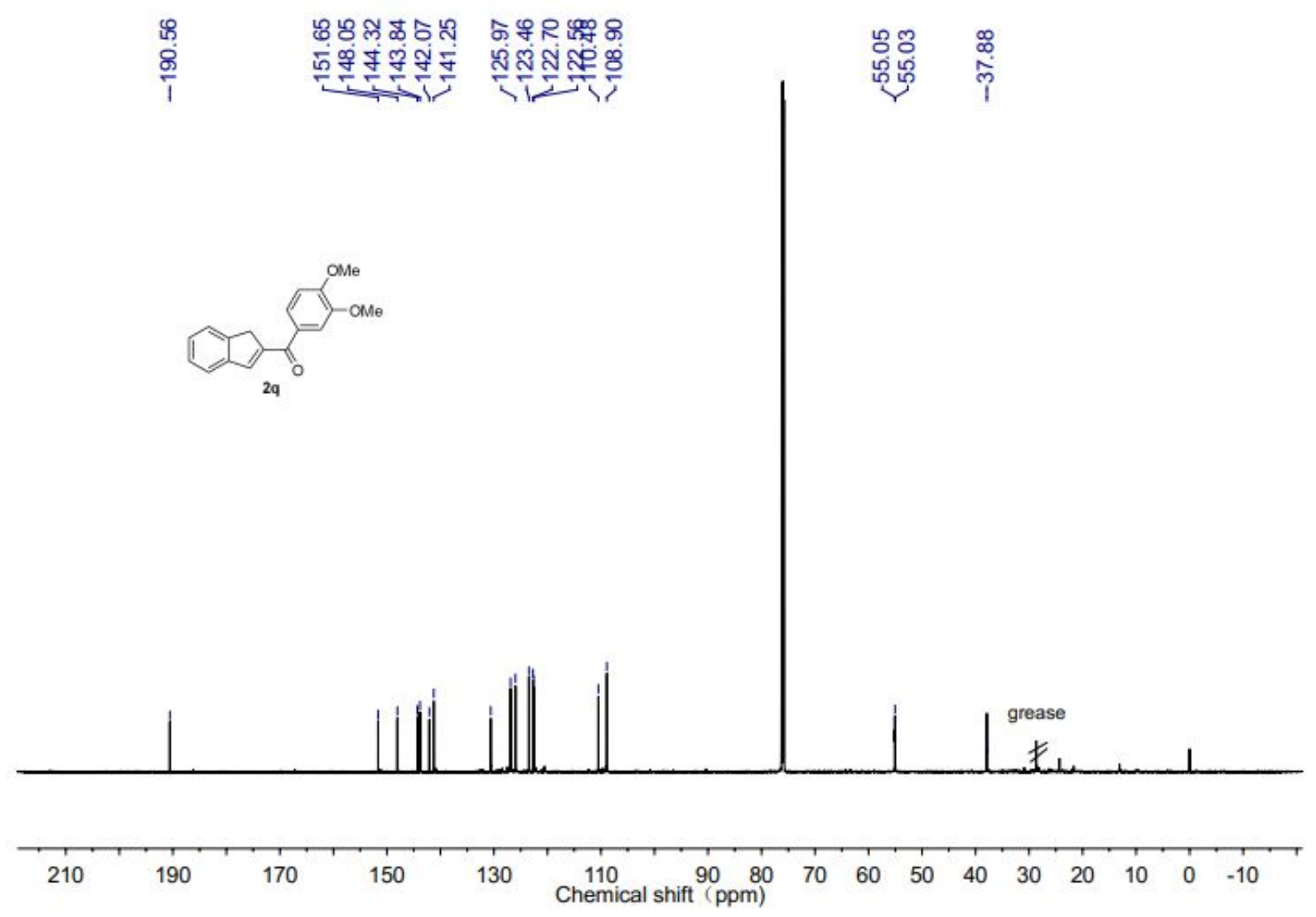

${ }^{13} \mathbf{C}\left\{{ }^{1} \mathbf{H}\right\}$ NMR $\left(151 \mathrm{MHz}, \mathrm{CDCl}_{3}, 298 \mathrm{~K}\right)$ of $\mathbf{2 q}$ 


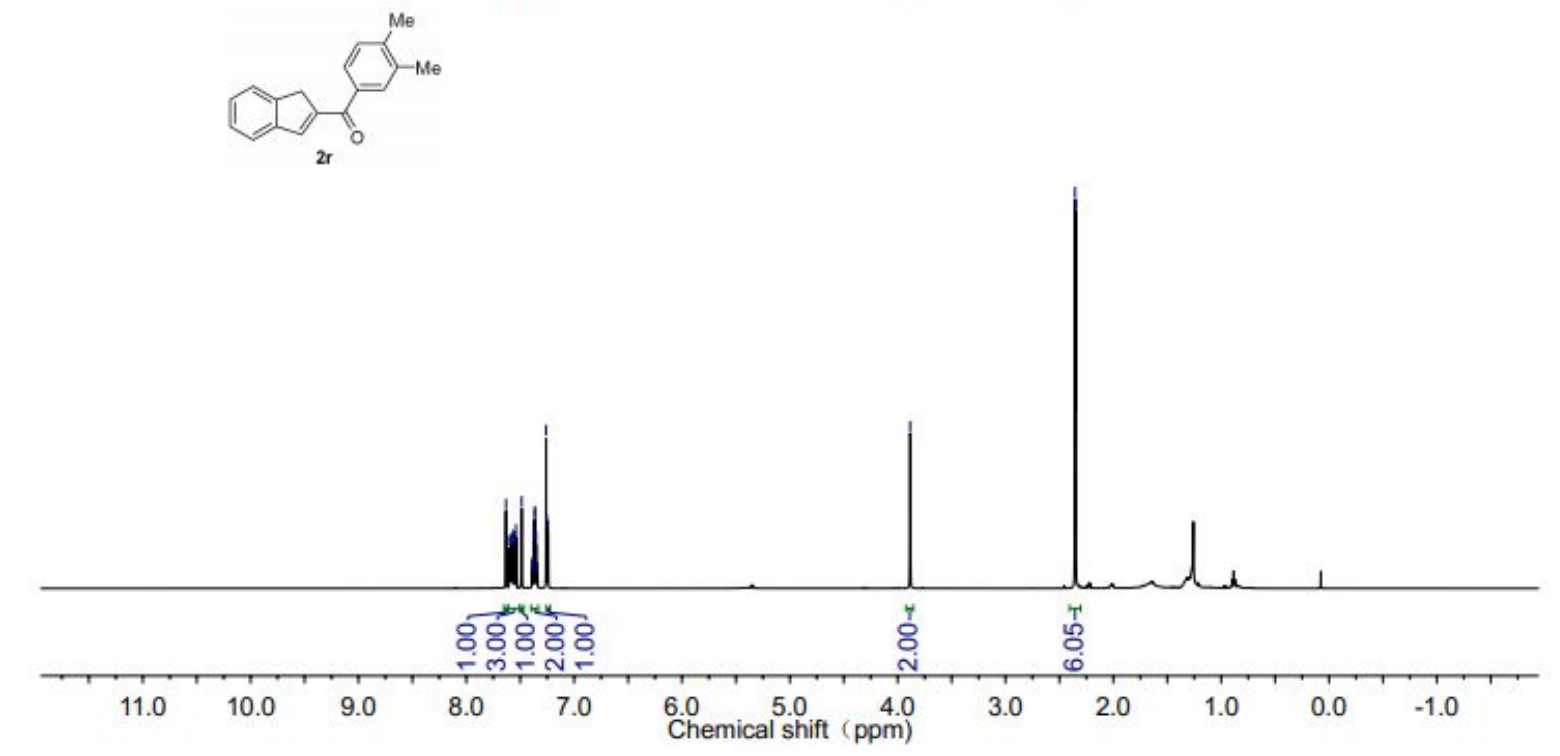

Wili
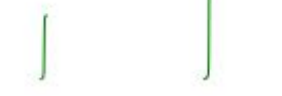

${ }^{\mathbf{1}} \mathbf{H}$ NMR $\left(600 \mathrm{MHz}, \mathrm{CDCl}_{3}, 298 \mathrm{~K}\right)$ of $\mathbf{2 r}$
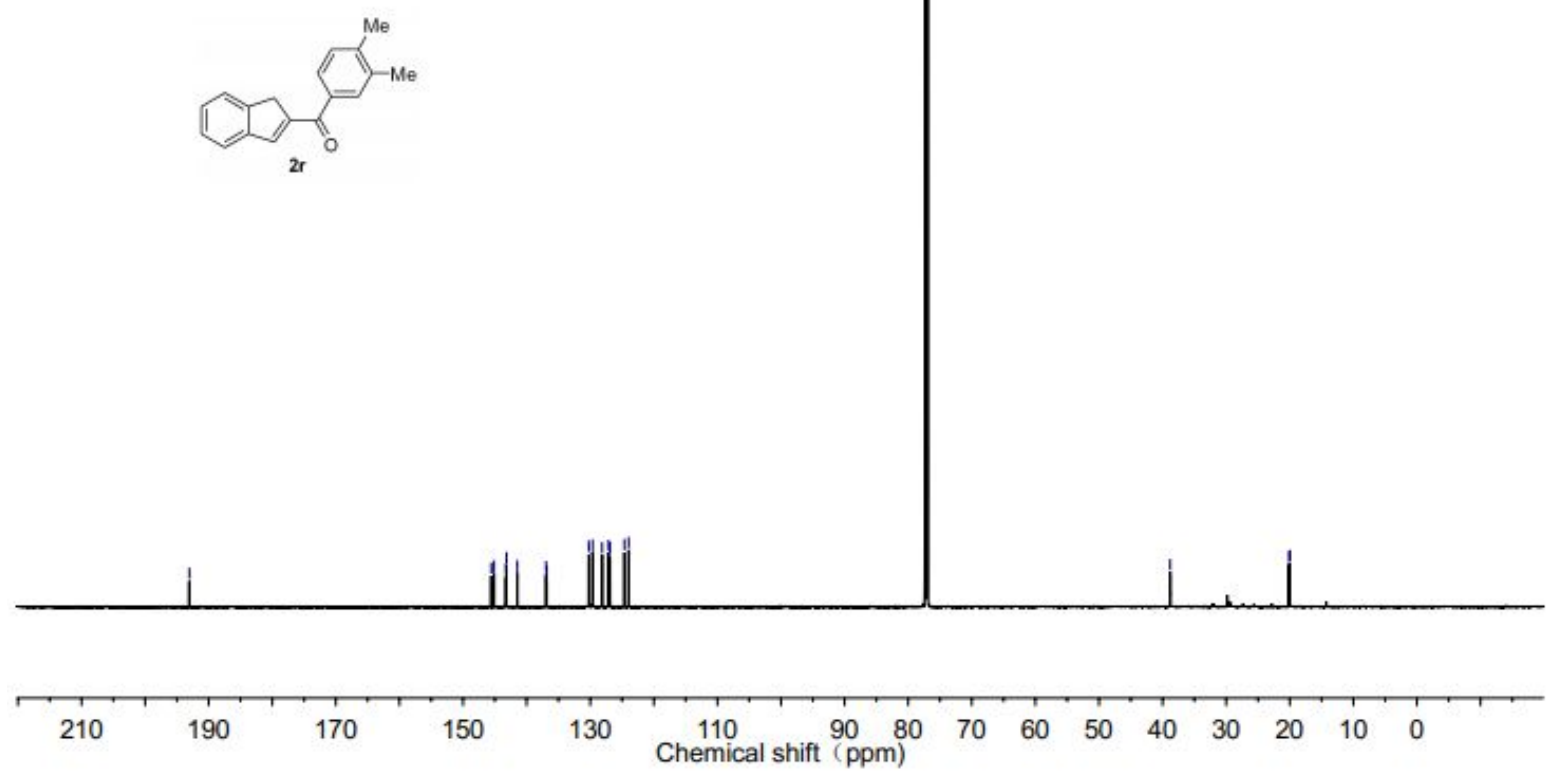

${ }^{13} \mathbf{C}\left\{{ }^{1} \mathbf{H}\right\}$ NMR $\left(151 \mathrm{MHz}, \mathrm{CDCl}_{3}, 298 \mathrm{~K}\right)$ of $\mathbf{2 r}$ 

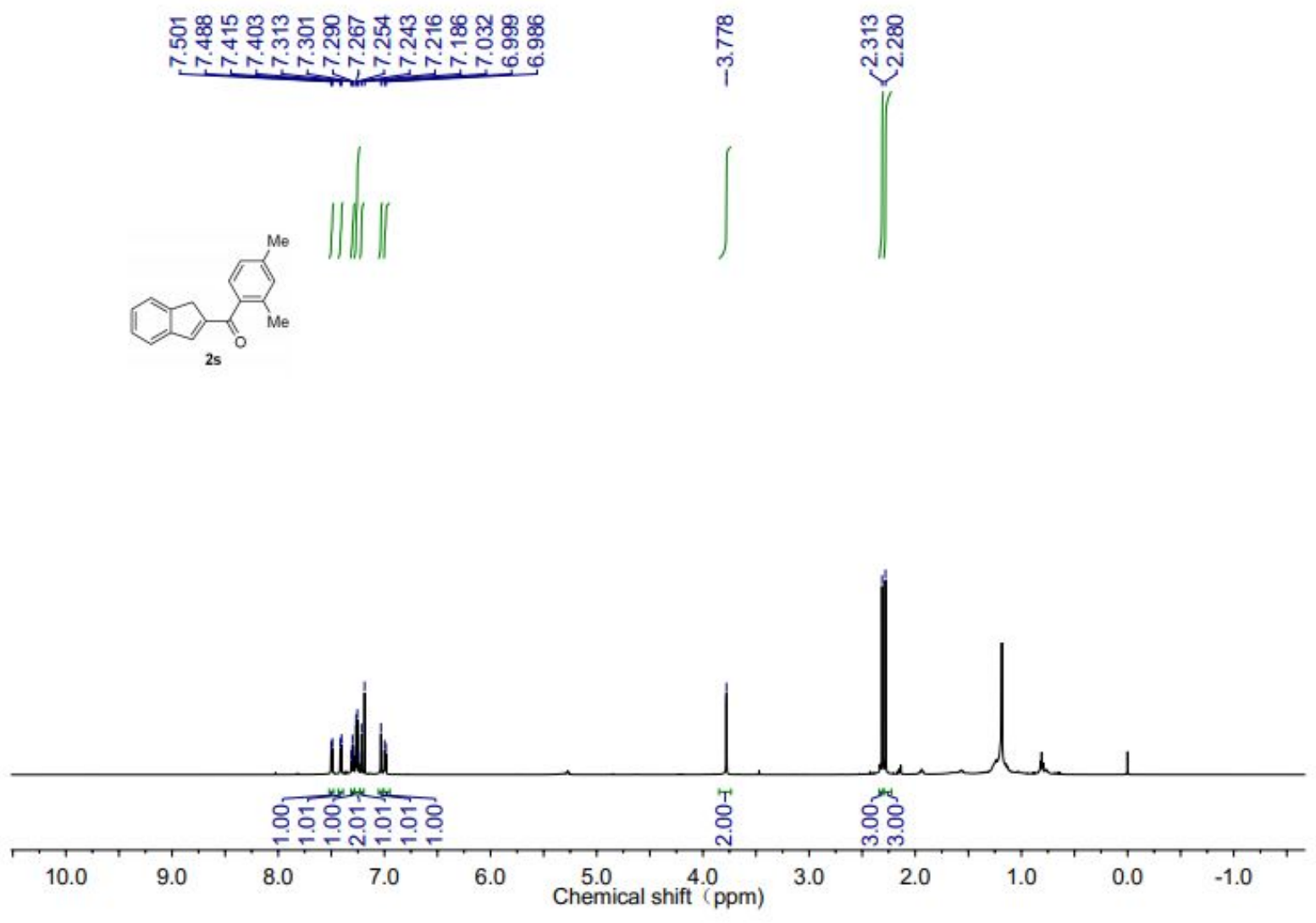

${ }^{\mathbf{1}} \mathbf{H}$ NMR $\left(600 \mathrm{MHz}, \mathrm{CDCl}_{3}, 298 \mathrm{~K}\right)$ of $\mathbf{2 s}$
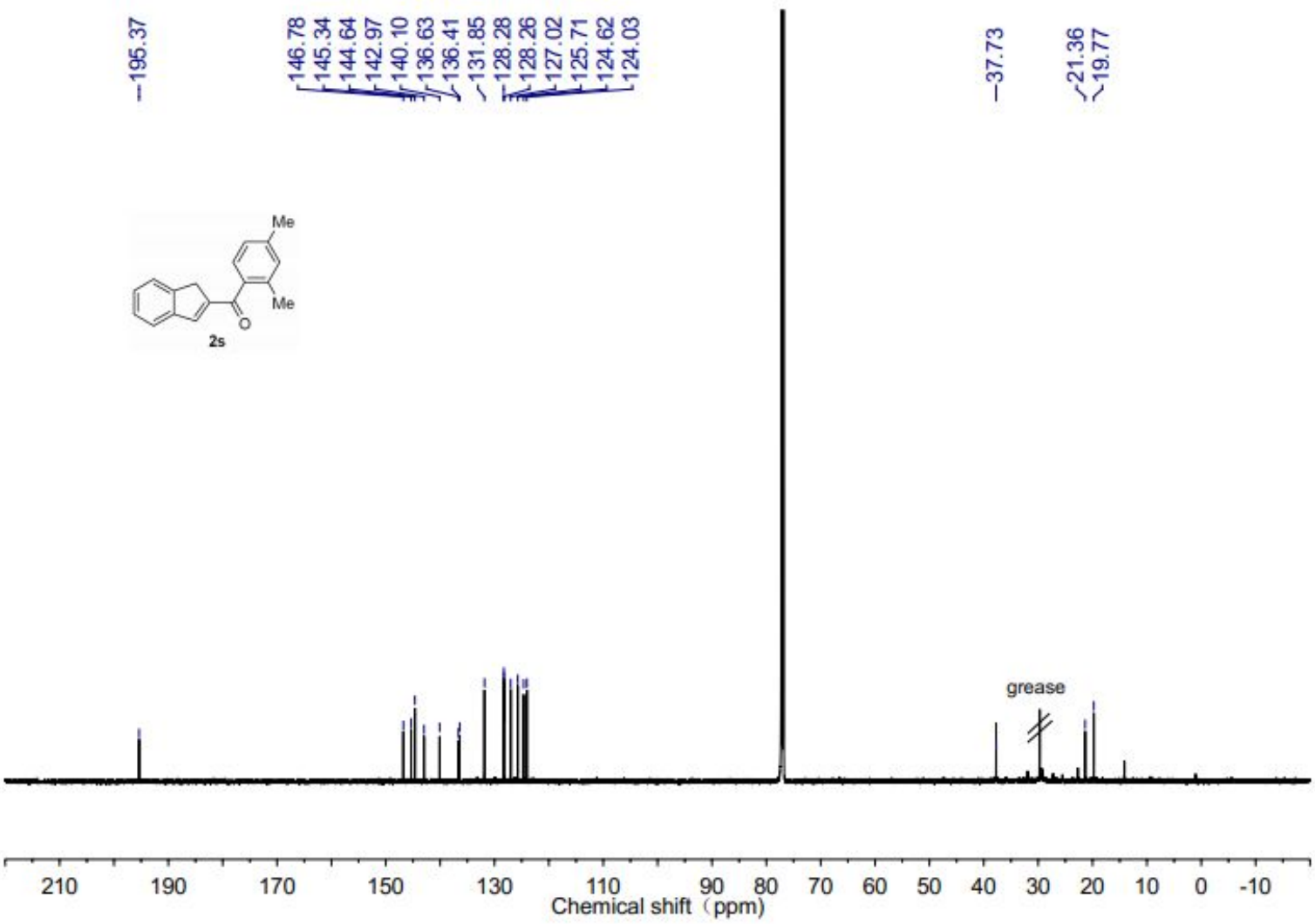

${ }^{13} \mathbf{C}\left\{{ }^{1} \mathbf{H}\right\}$ NMR $\left(151 \mathrm{MHz}, \mathrm{CDCl}_{3}, 298 \mathrm{~K}\right)$ of $\mathbf{2 s}$ 

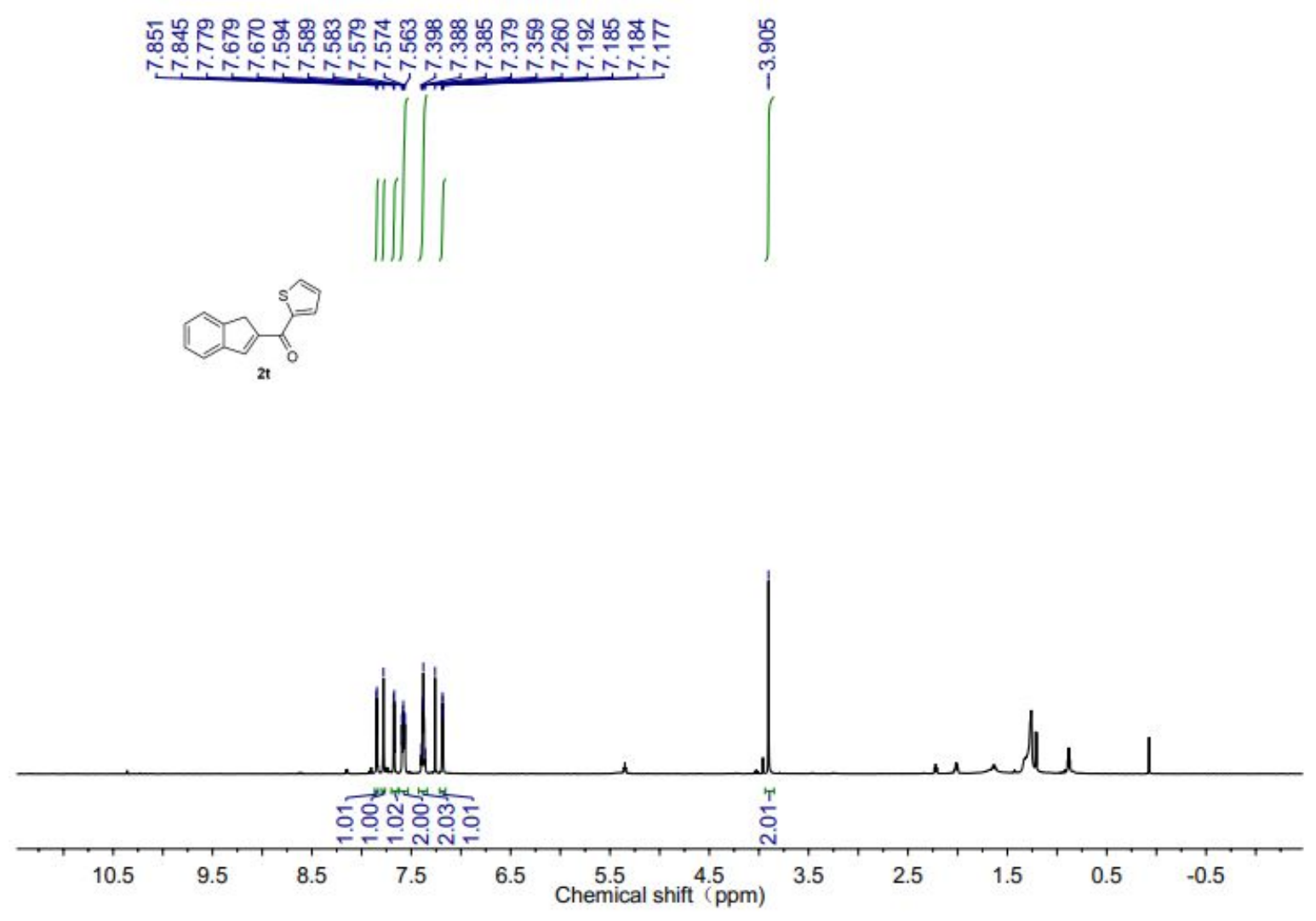

${ }^{1} \mathbf{H}$ NMR $\left(600 \mathrm{MHz}, \mathrm{CDCl}_{3}, 298 \mathrm{~K}\right)$ of $\mathbf{2 t}$

\begin{tabular}{|c|c|c|}
\hline$\stackrel{\bar{\emptyset}}{\frac{\infty}{1}}$ & 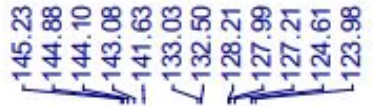 & 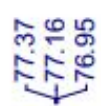 \\
\hline
\end{tabular}

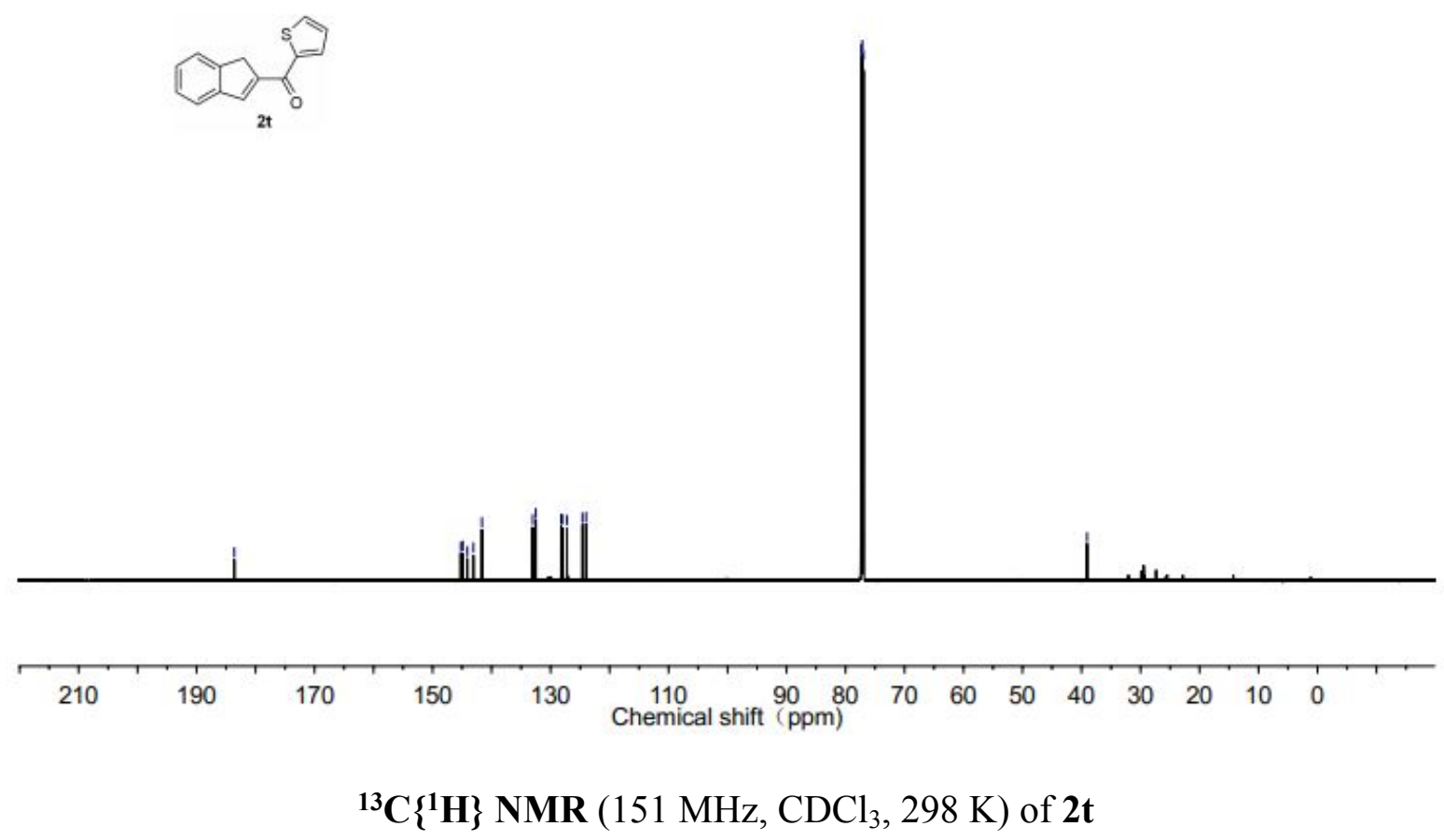



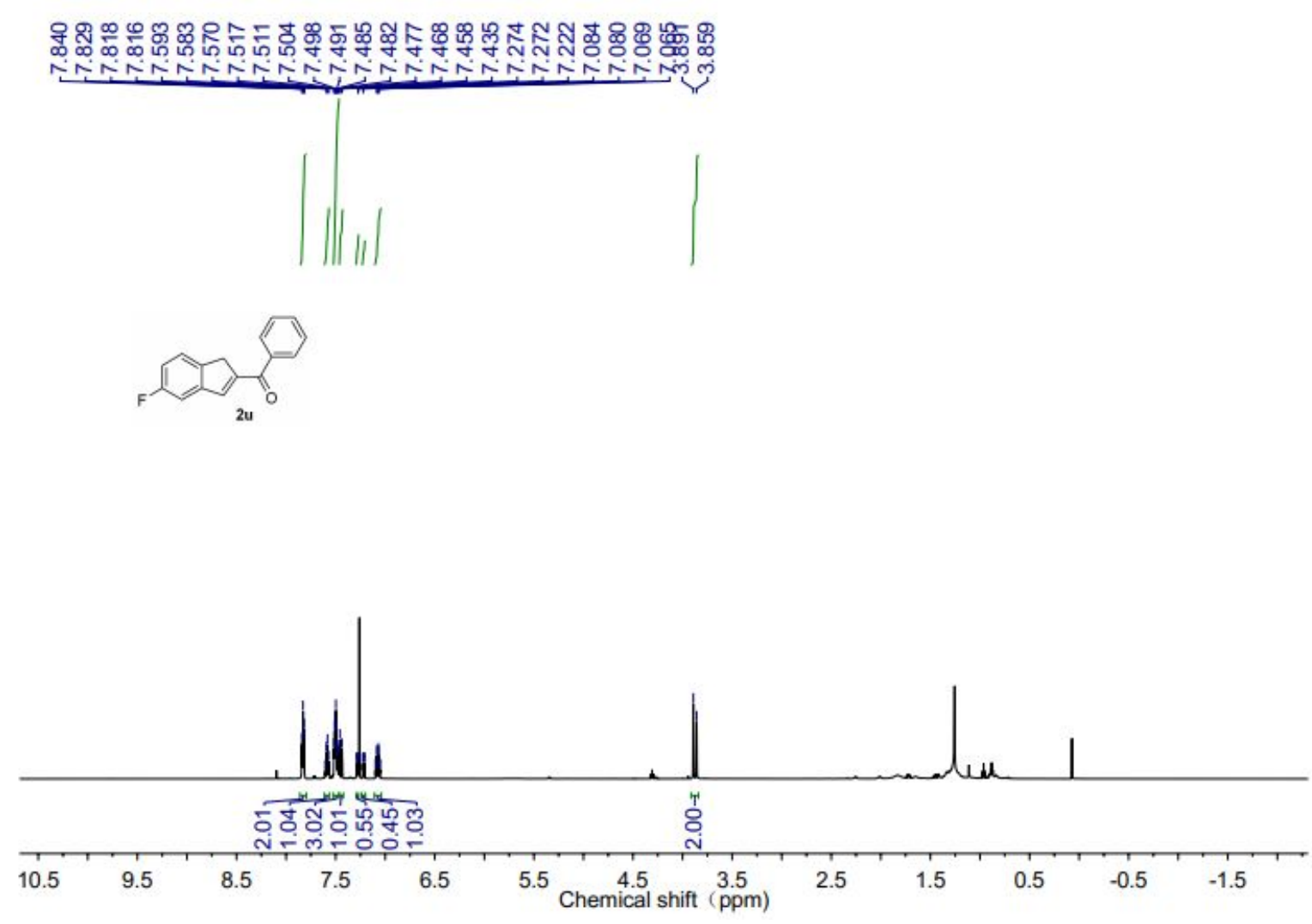

${ }^{1} \mathbf{H}$ NMR $\left(600 \mathrm{MHz}, \mathrm{CDCl}_{3}, 298 \mathrm{~K}\right)$ of $\mathbf{2 u}$
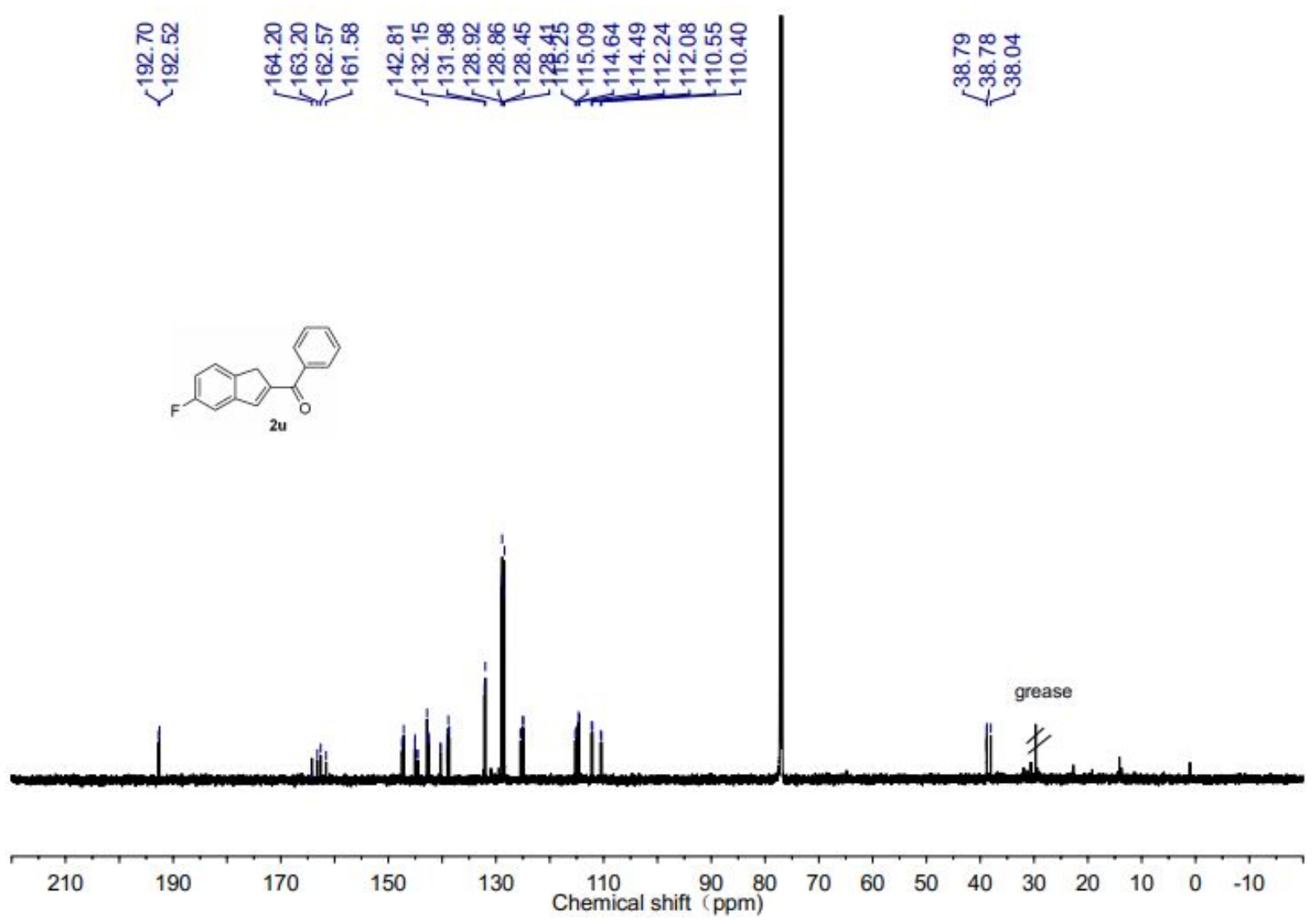

${ }^{13} \mathbf{C}\left\{{ }^{1} \mathbf{H}\right\}$ NMR $\left(151 \mathrm{MHz}, \mathrm{CDCl}_{3}, 298 \mathrm{~K}\right)$ of $\mathbf{2 u}$ 


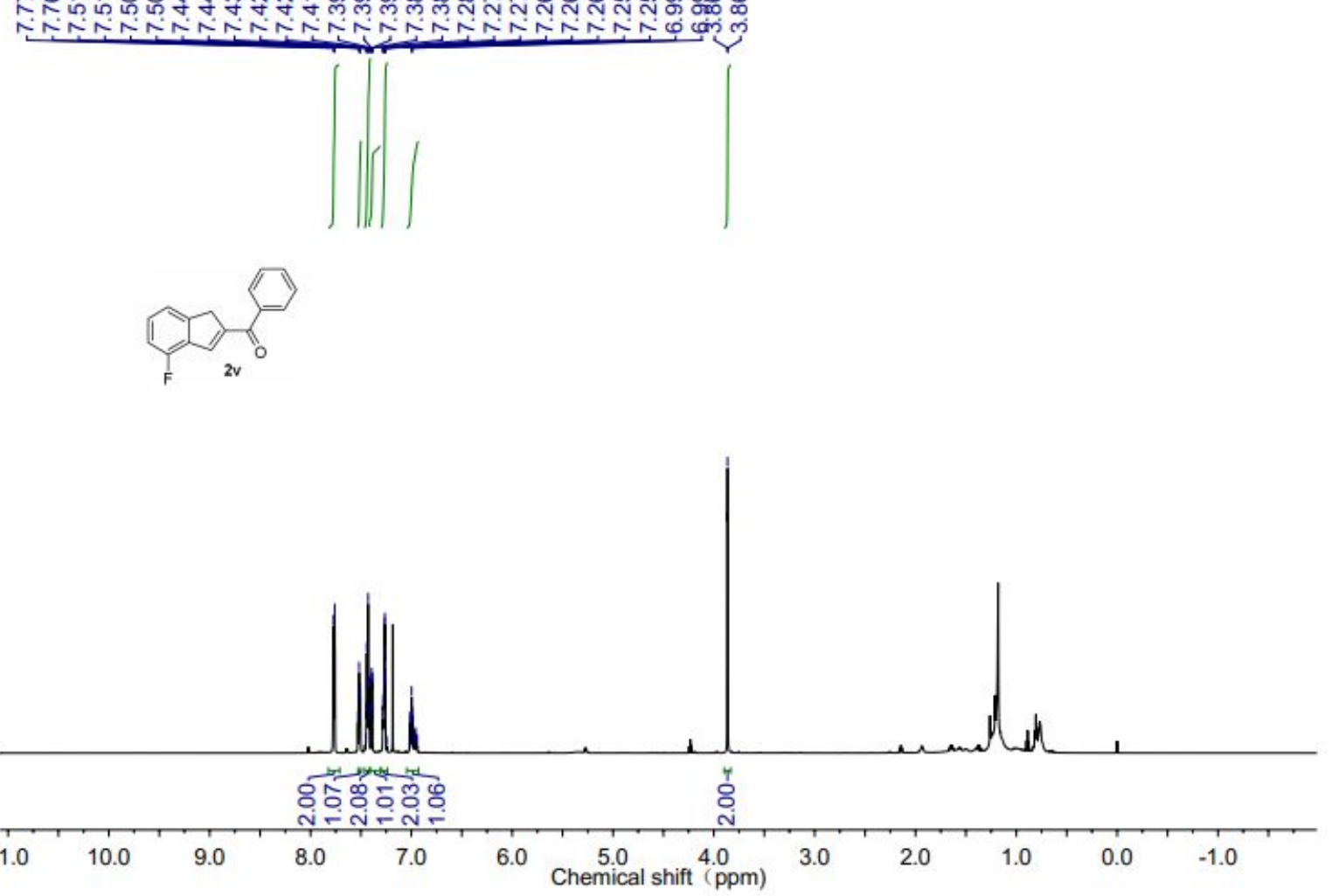

${ }^{1} \mathbf{H}$ NMR $\left(600 \mathrm{MHz}, \mathrm{CDCl}_{3}, 298 \mathrm{~K}\right)$ of $\mathbf{2 v}$
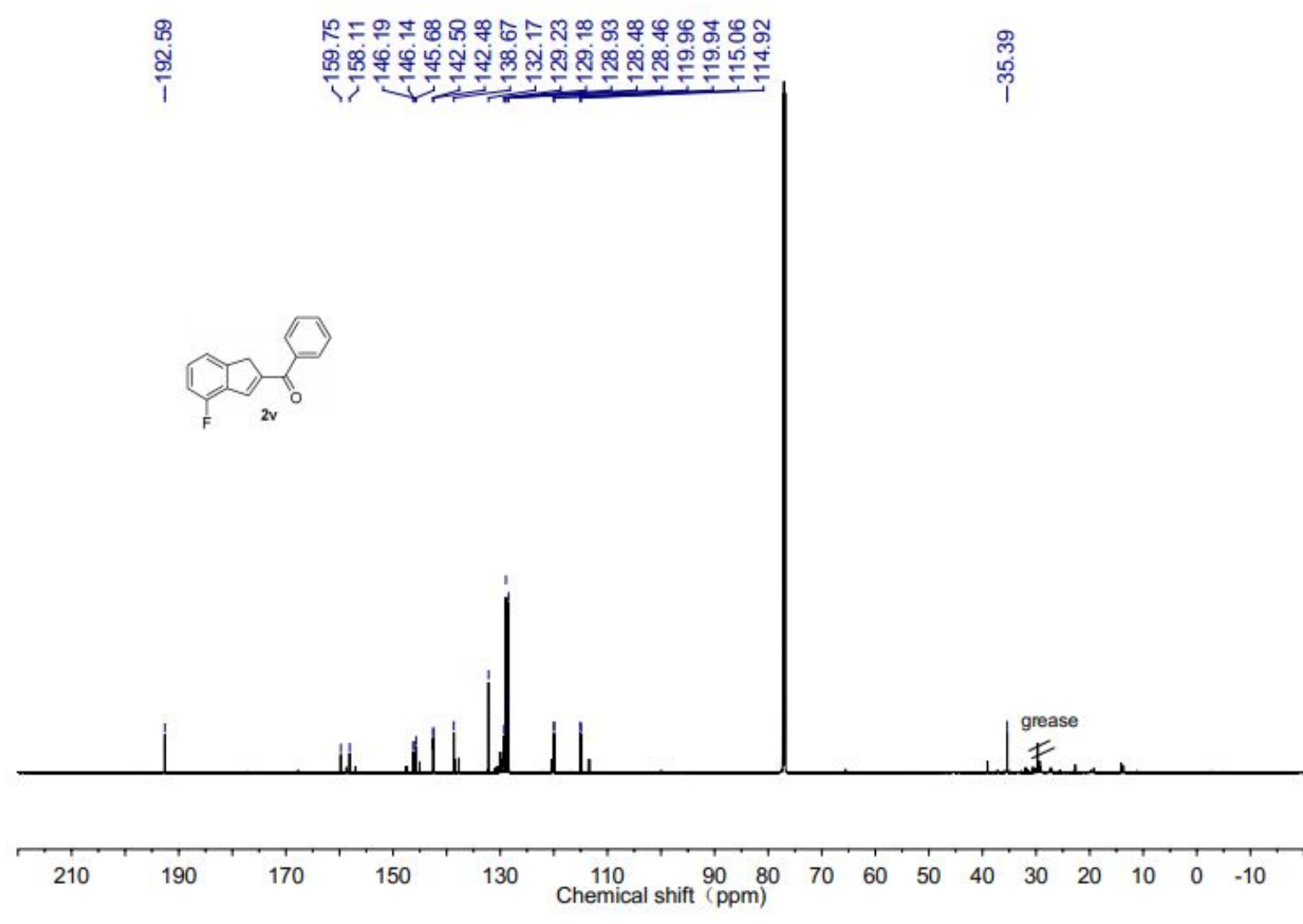

${ }^{13} \mathbf{C}\left\{{ }^{1} \mathbf{H}\right\}$ NMR $\left(151 \mathrm{MHz}, \mathrm{CDCl}_{3}, 298 \mathrm{~K}\right)$ of $\mathbf{2 v}$ 


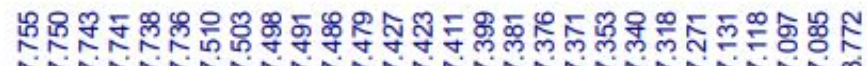

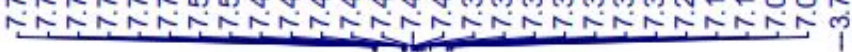
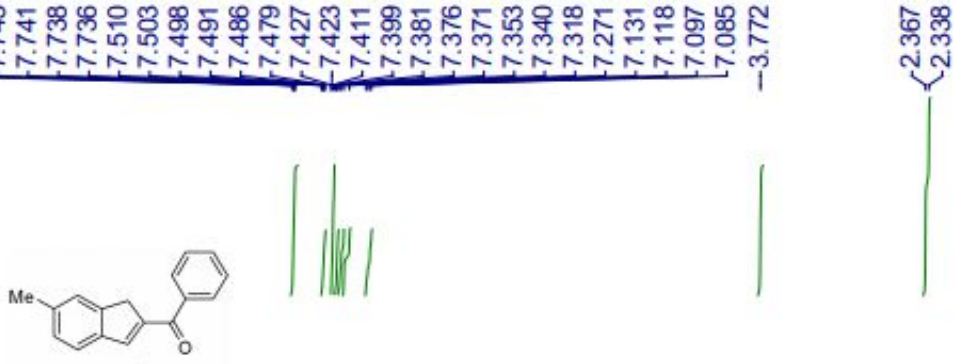

$2 w$

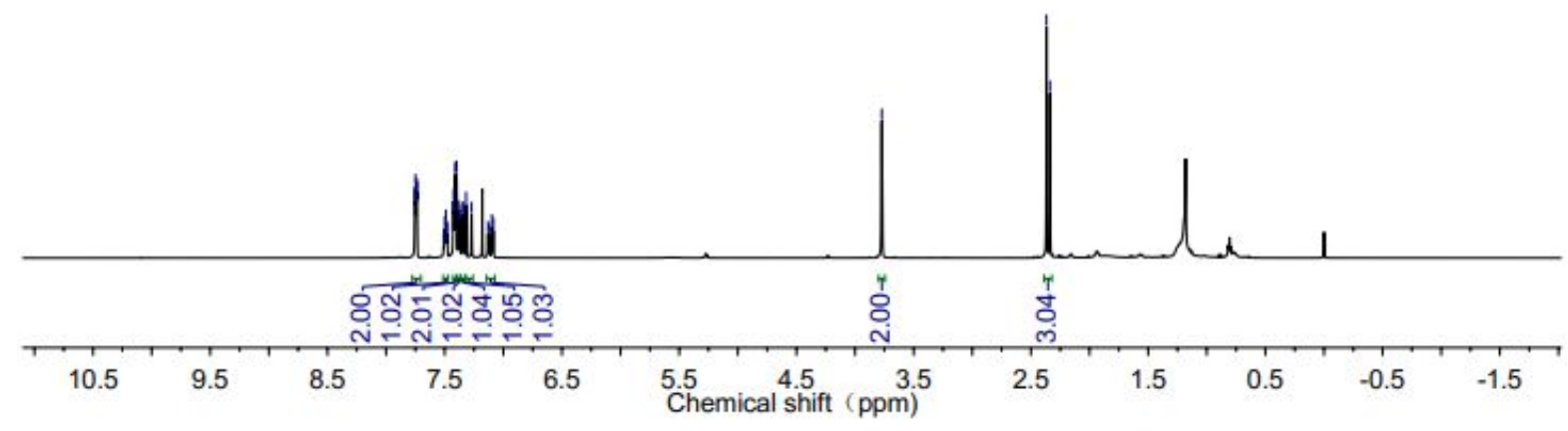

${ }^{1} \mathbf{H}$ NMR $\left(600 \mathrm{MHz}, \mathrm{CDCl}_{3}, 298 \mathrm{~K}\right)$ of $\mathbf{2} \mathbf{w}$

க ธ

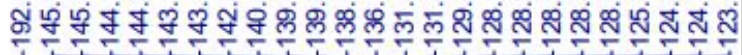

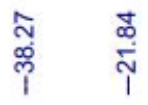
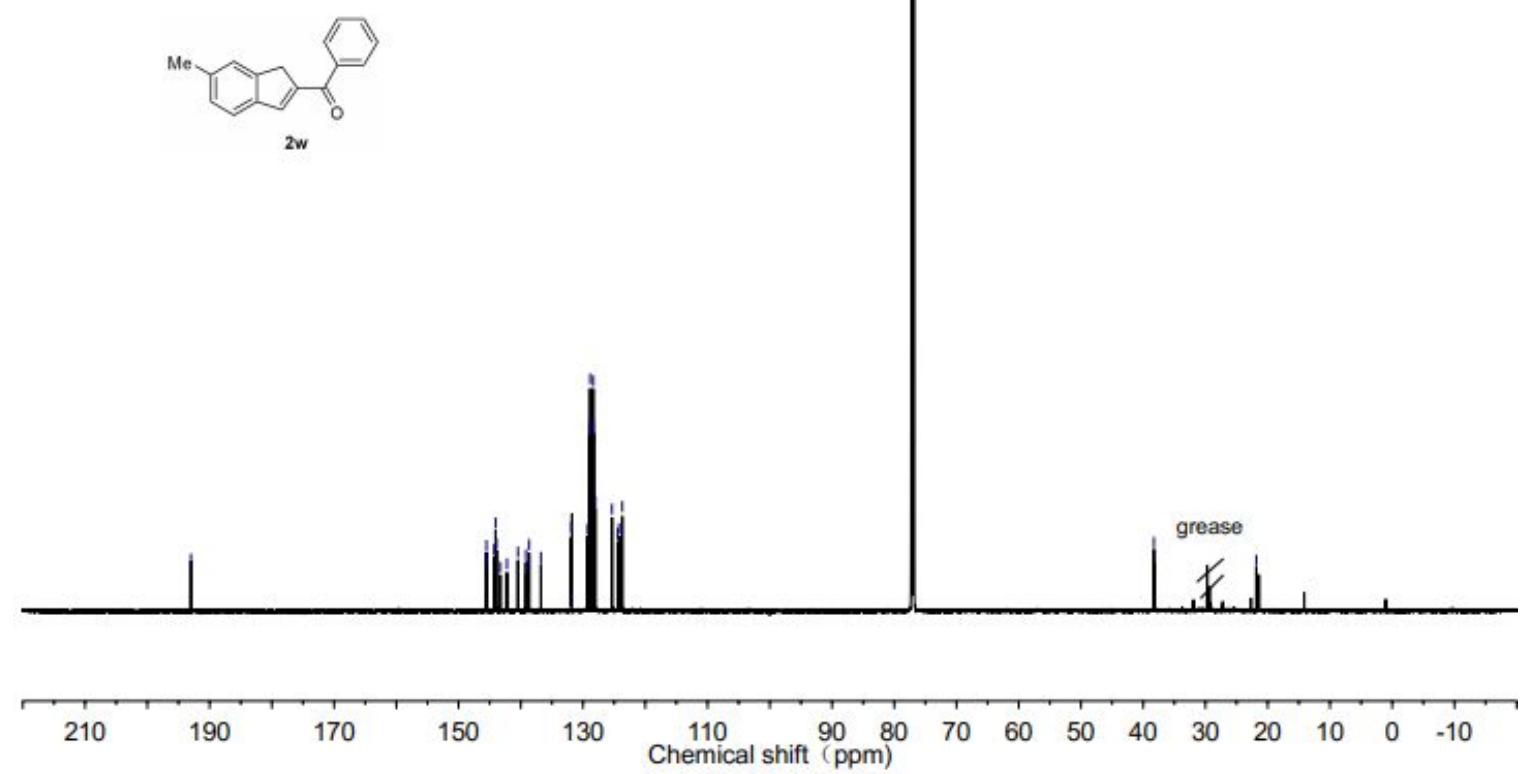

${ }^{13} \mathbf{C}\left\{{ }^{1} \mathbf{H}\right\}$ NMR $\left(151 \mathrm{MHz}, \mathrm{CDCl}_{3}, 298 \mathrm{~K}\right)$ of $\mathbf{2 w}$ 


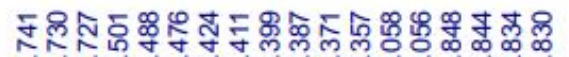

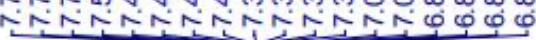

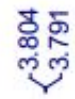

||||
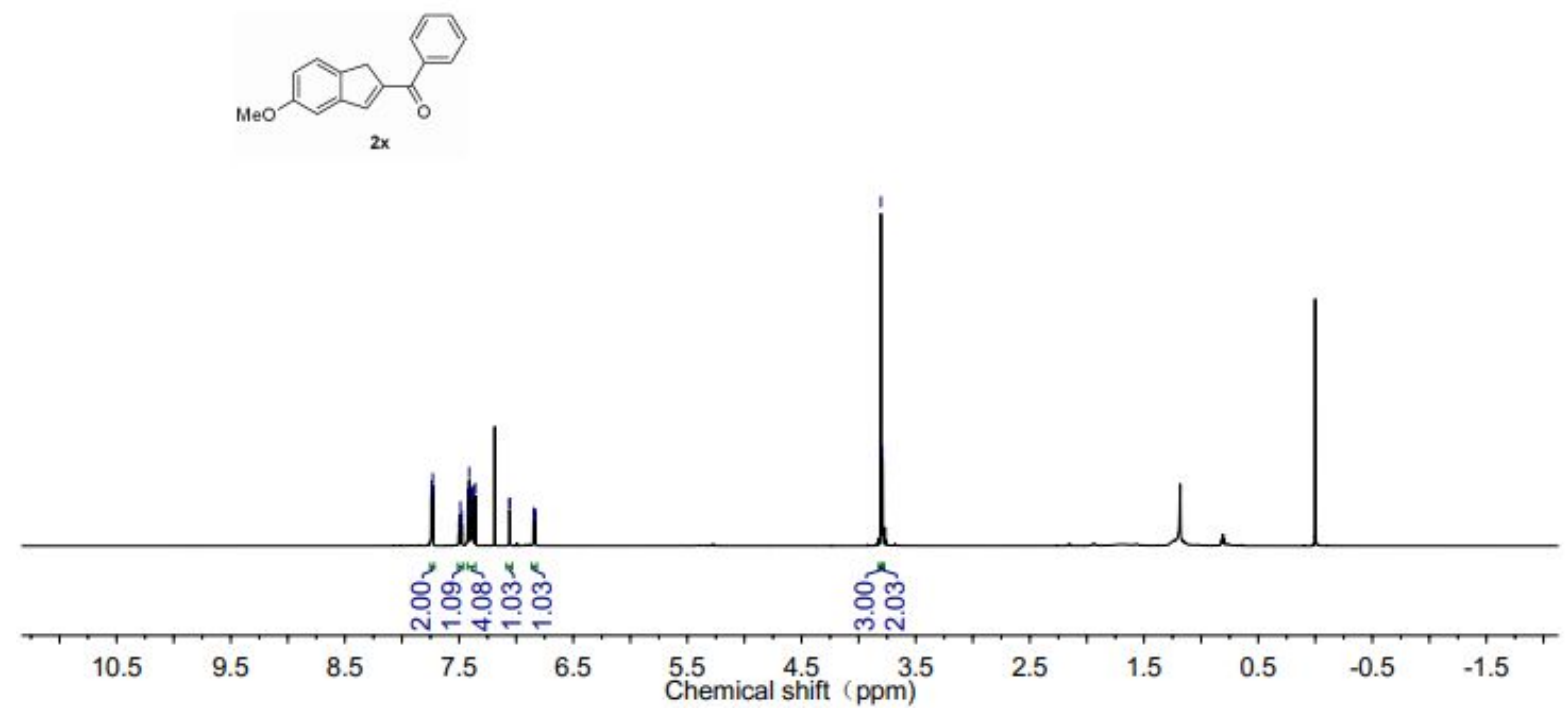

${ }^{1} \mathbf{H}$ NMR $\left(600 \mathrm{MHz}, \mathrm{CDCl}_{3}, 298 \mathrm{~K}\right)$ of $\mathbf{2 x}$

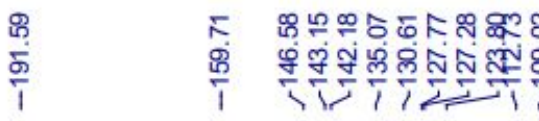

草
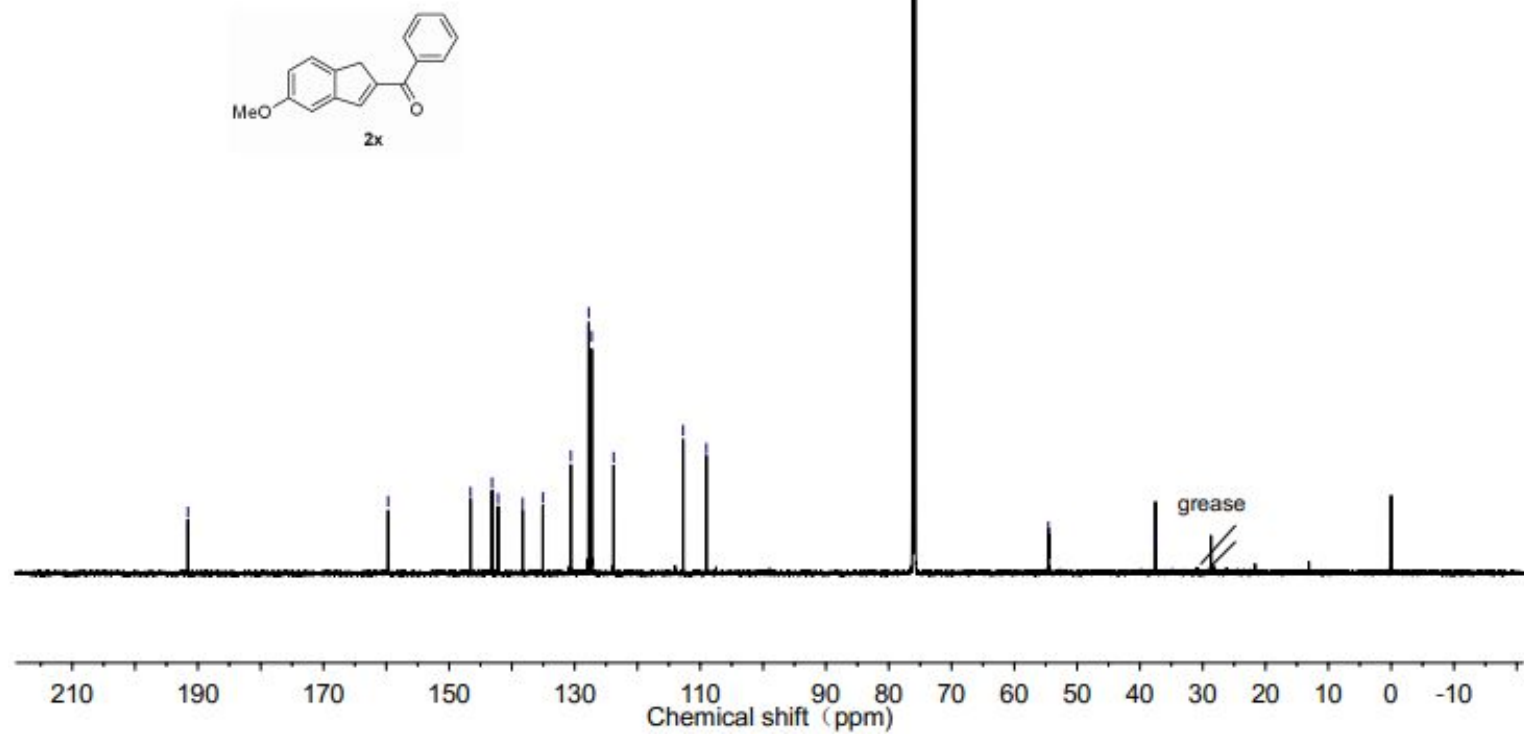

${ }^{13} \mathbf{C}\left\{{ }^{1} \mathbf{H}\right\} \mathbf{N M R}\left(151 \mathrm{MHz}, \mathrm{CDCl}_{3}, 298 \mathrm{~K}\right)$ of $\mathbf{2 x}$ 

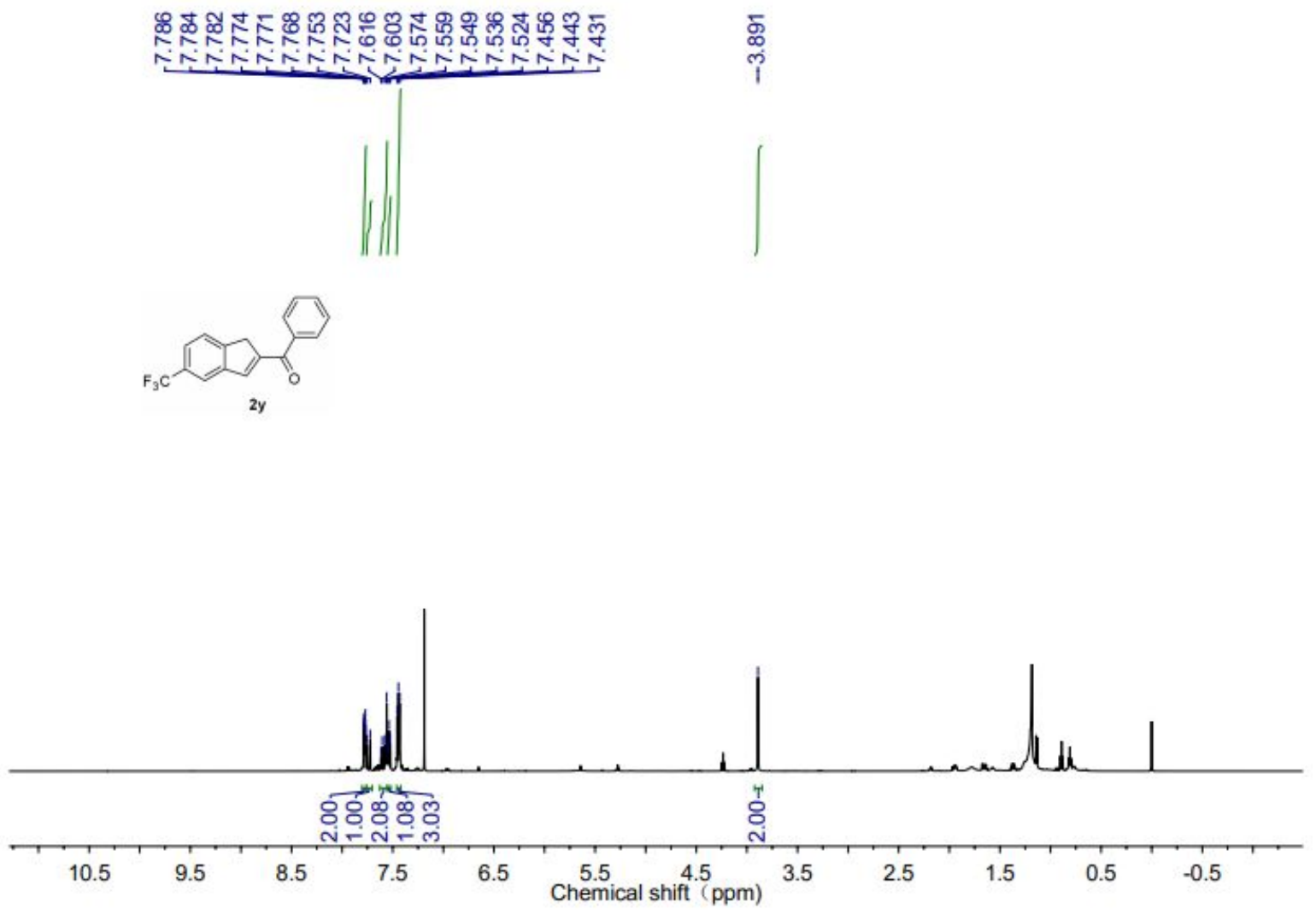

${ }^{1} \mathbf{H}$ NMR $\left(600 \mathrm{MHz}, \mathrm{CDCl}_{3}, 298 \mathrm{~K}\right)$ of $\mathbf{2 y}$
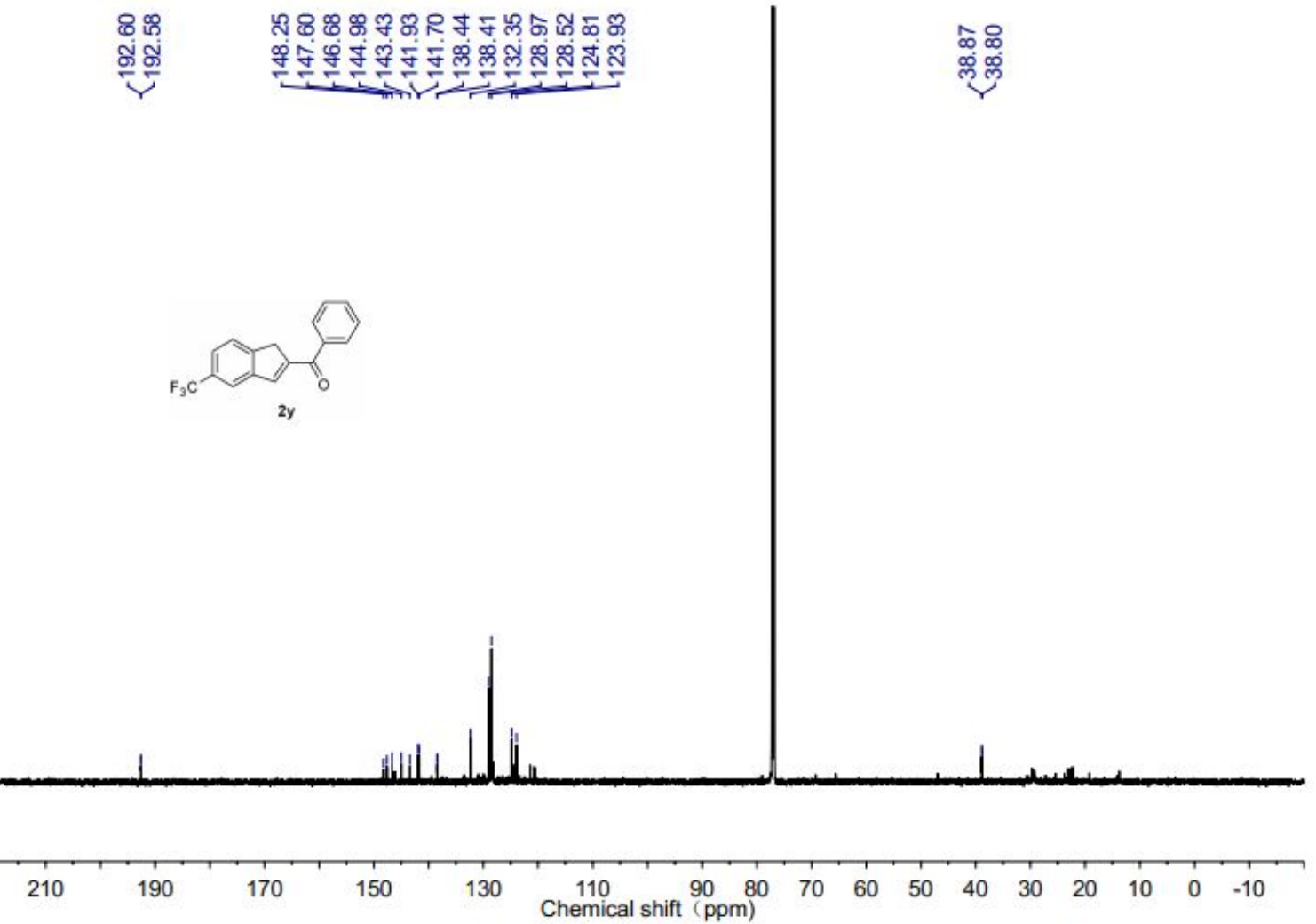

${ }^{13} \mathbf{C}\left\{{ }^{1} \mathbf{H}\right\} \mathbf{N M R}\left(151 \mathrm{MHz}, \mathrm{CDCl}_{3}, 298 \mathrm{~K}\right)$ of $\mathbf{2 y}$ 

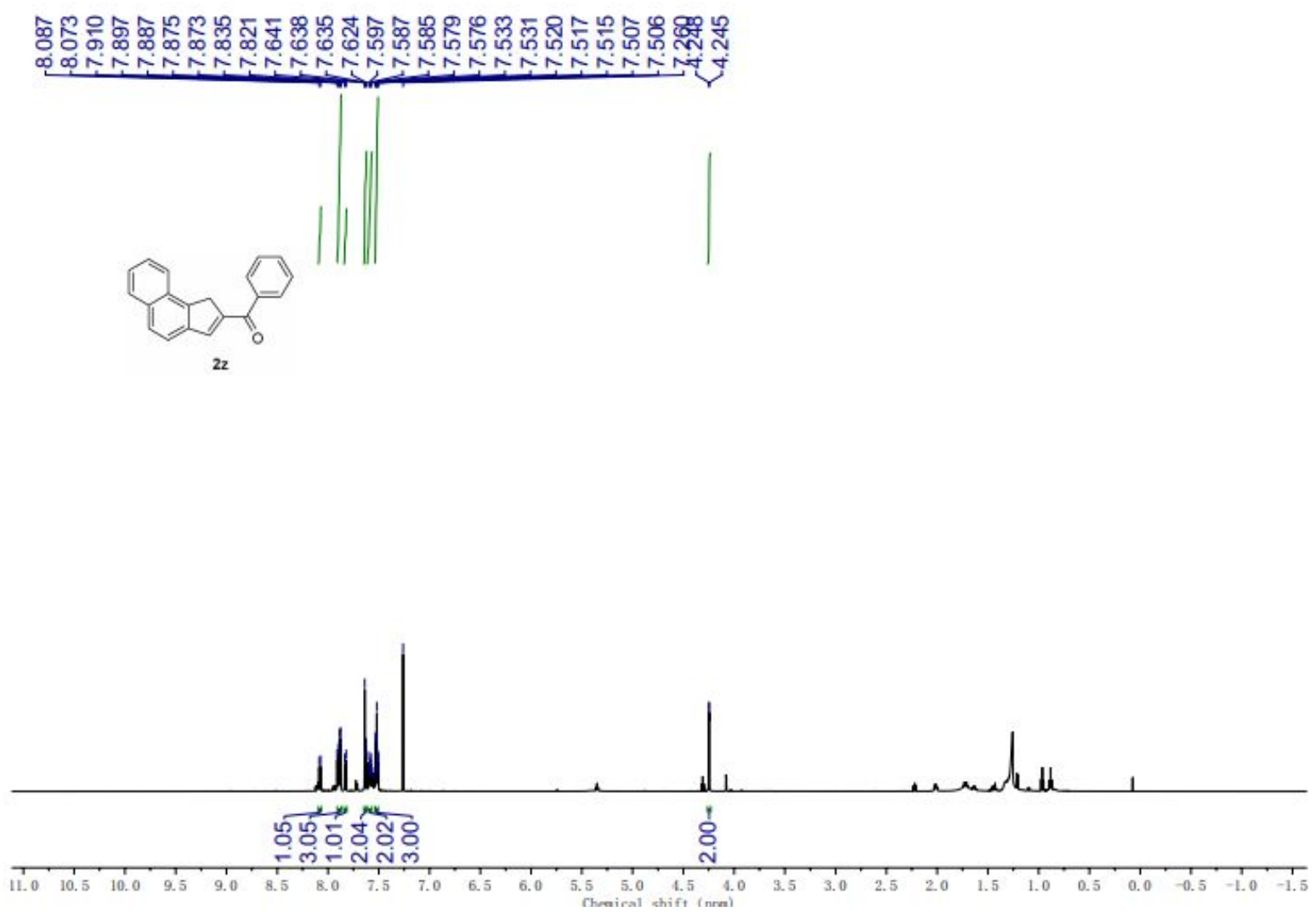

${ }^{1} \mathbf{H}$ NMR $\left(600 \mathrm{MHz}, \mathrm{CDCl}_{3}, 298 \mathrm{~K}\right)$ of $\mathbf{2 z}$

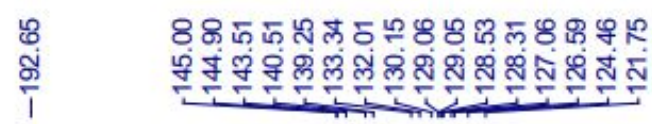
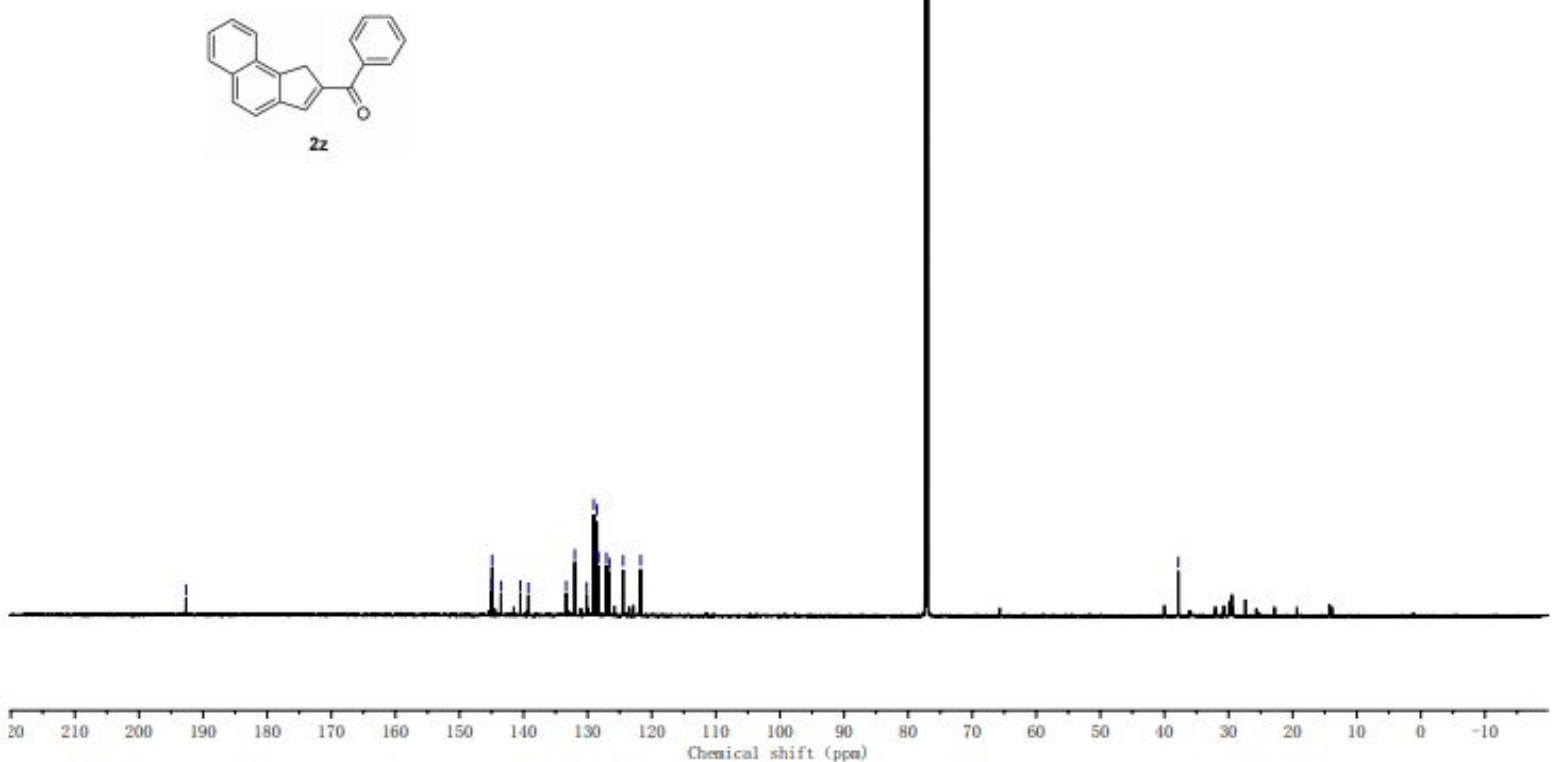

${ }^{13} \mathbf{C}\left\{{ }^{1} \mathbf{H}\right\}$ NMR $\left(151 \mathrm{MHz}, \mathrm{CDCl}_{3}, 298 \mathrm{~K}\right)$ of $\mathbf{2 z}$ 

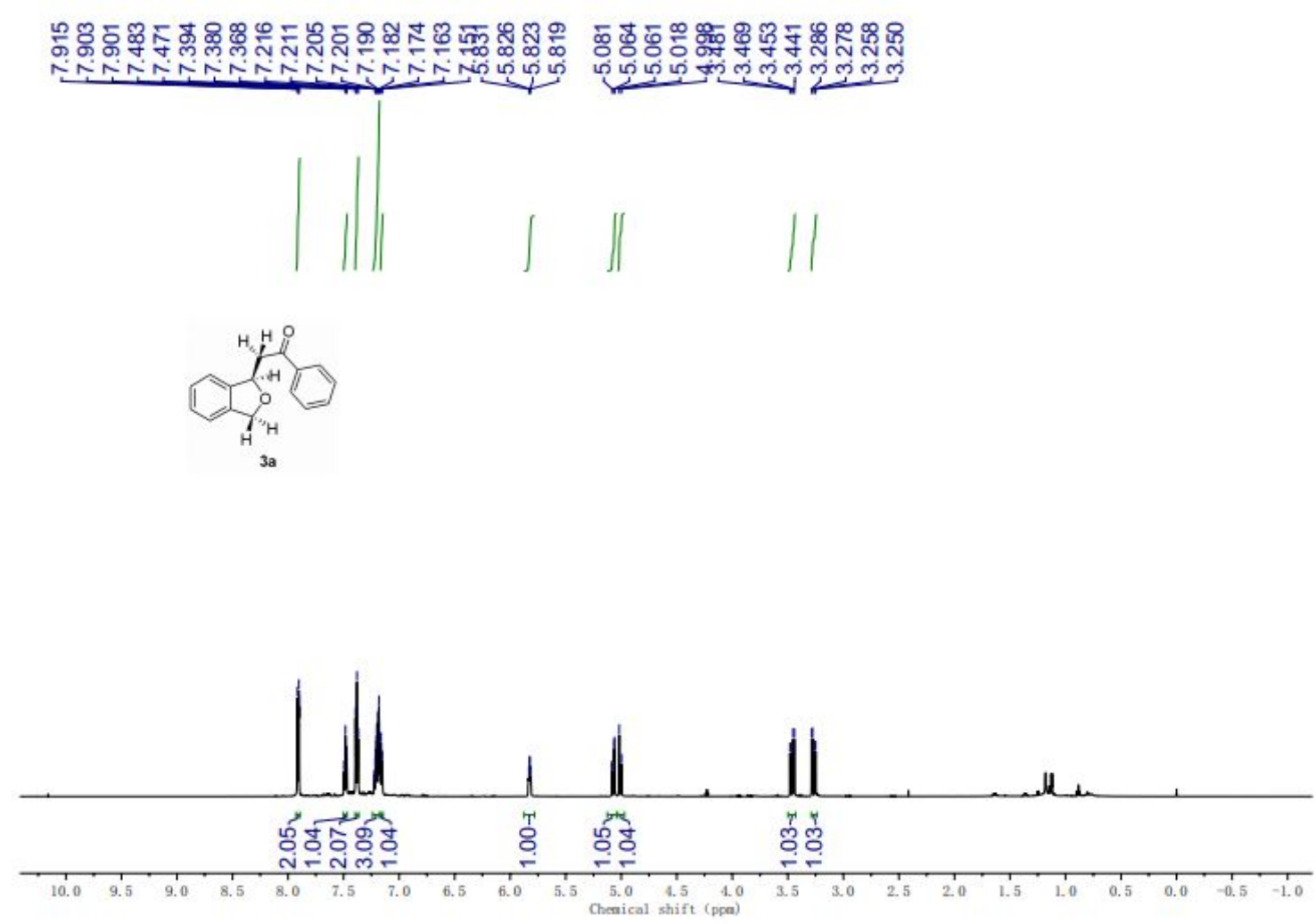

${ }^{1} \mathbf{H}$ NMR $\left(600 \mathrm{MHz}, \mathrm{CDCl}_{3}, 298 \mathrm{~K}\right)$ of $\mathbf{3 a}$
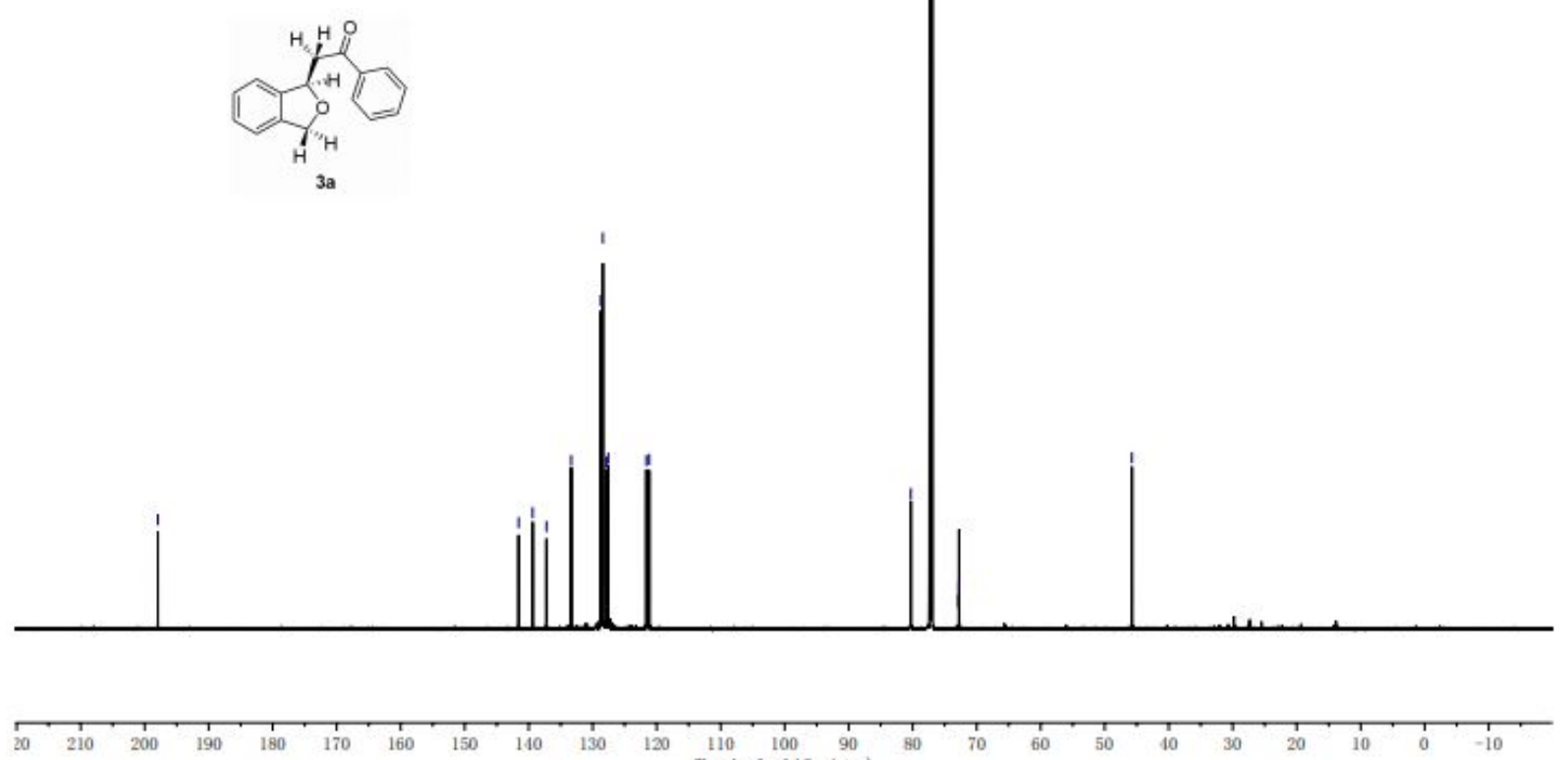

${ }^{13} \mathbf{C}\left\{{ }^{1} \mathbf{H}\right\}$ NMR (151 MHz, $\left.\mathrm{CDCl}_{3}, 298 \mathrm{~K}\right)$ of $\mathbf{3 a}$ 


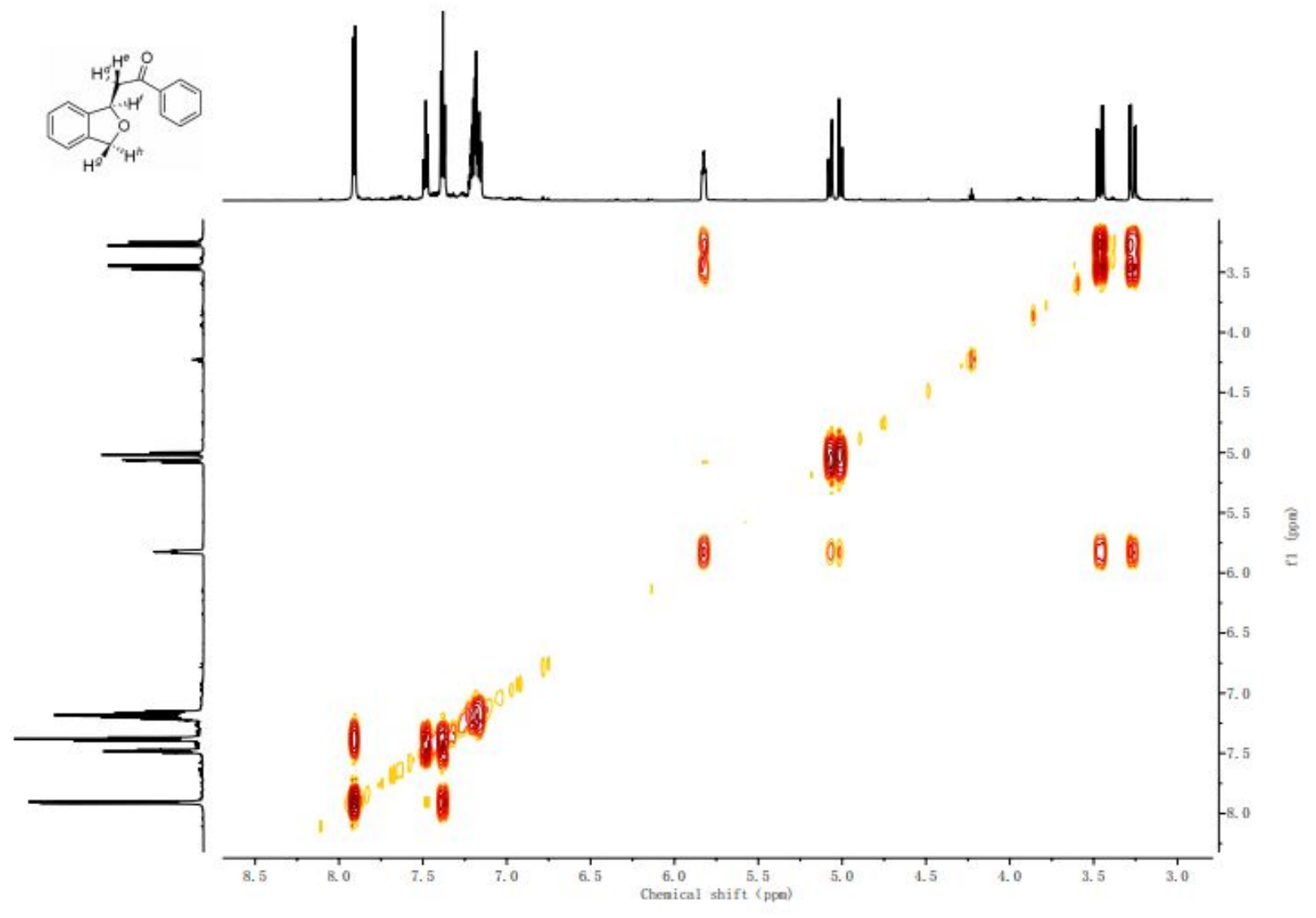

${ }^{1} \mathrm{H}-{ }^{1} \mathrm{H}$ COSY of $\mathbf{3 a}$

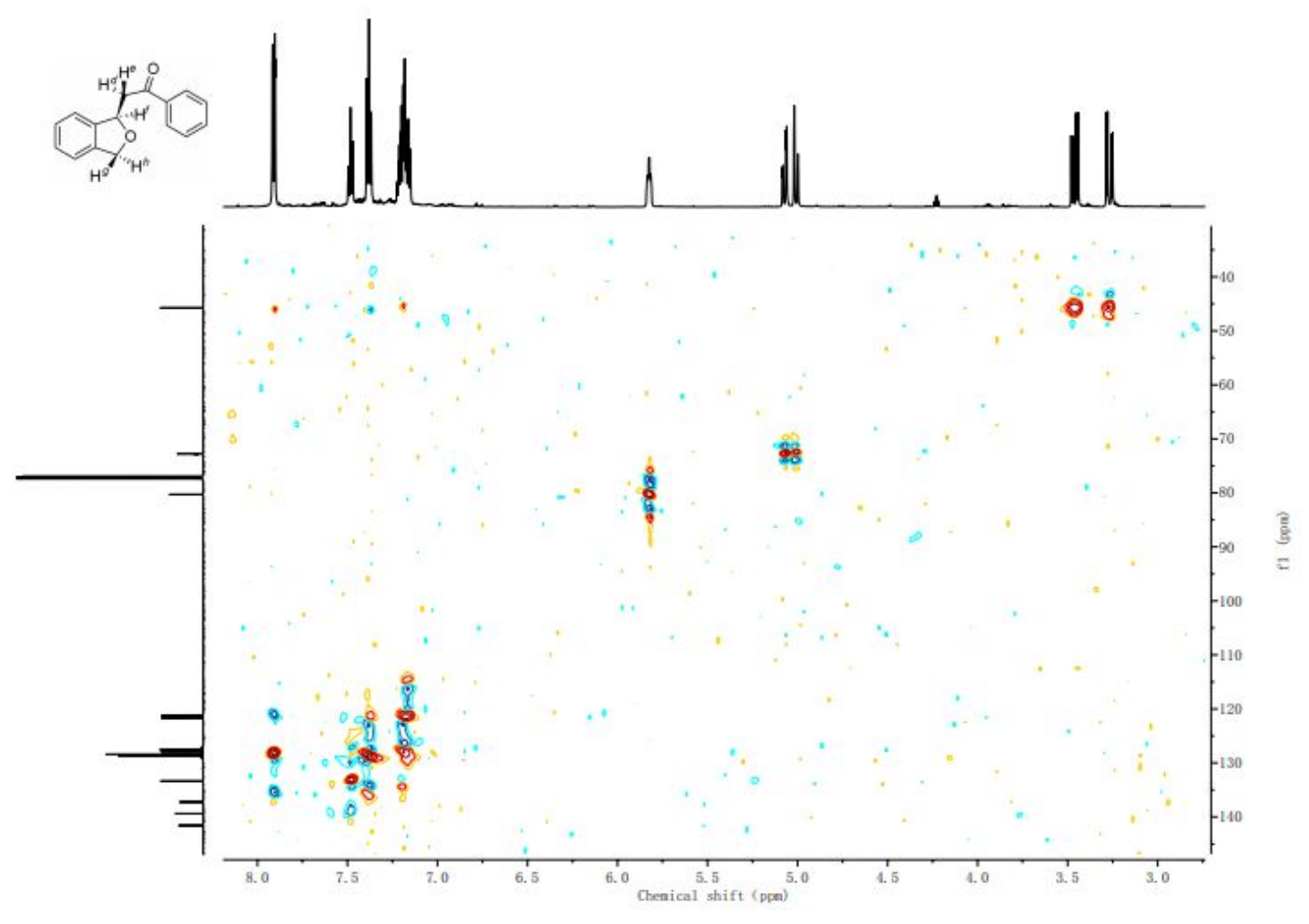

${ }^{1} \mathrm{H}-{ }^{13} \mathrm{C}$ HSQC of $3 \mathrm{a}$ 


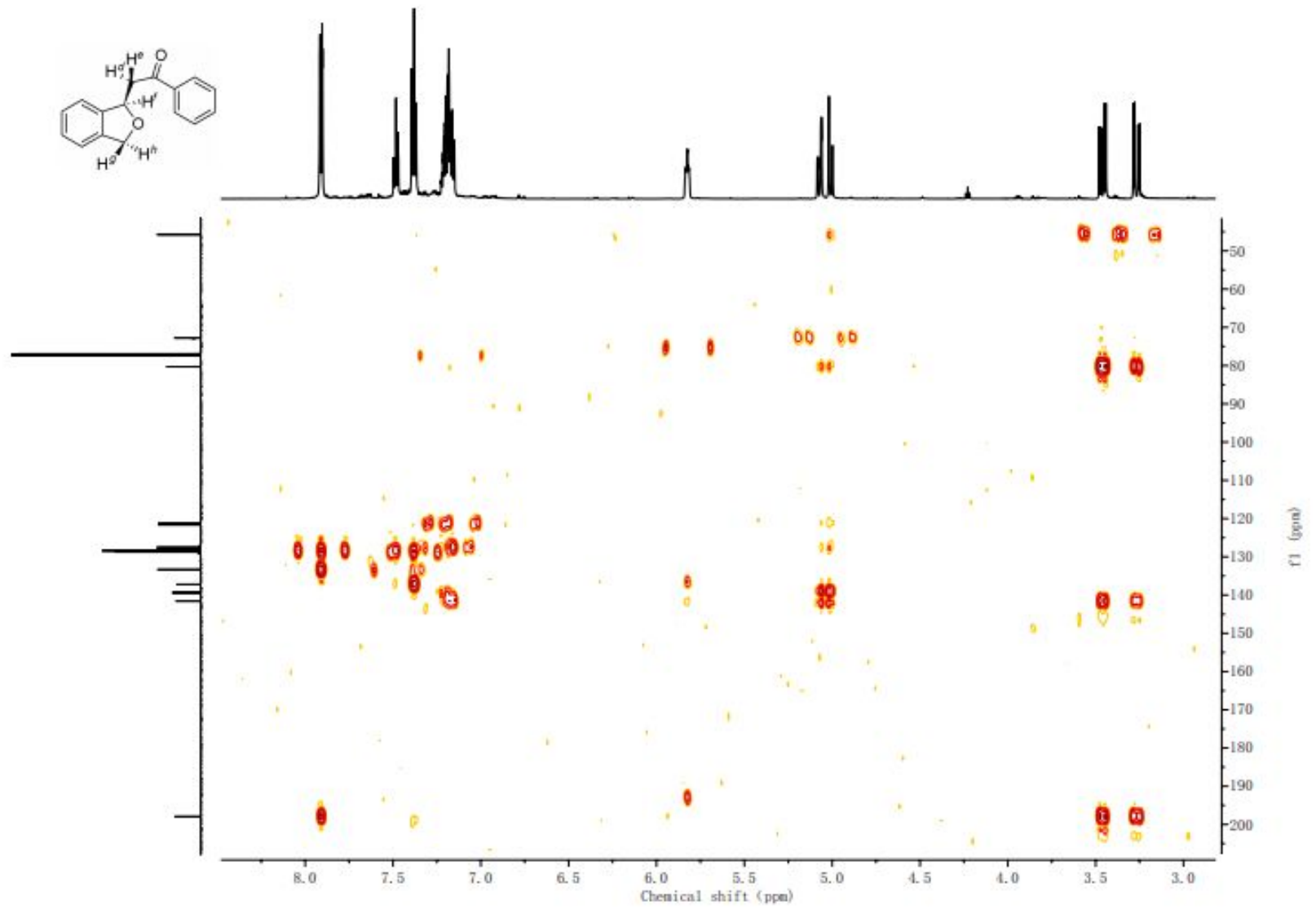

${ }^{1} \mathrm{H}-{ }^{13} \mathrm{C}$ HMBC of $3 \mathrm{a}$ 

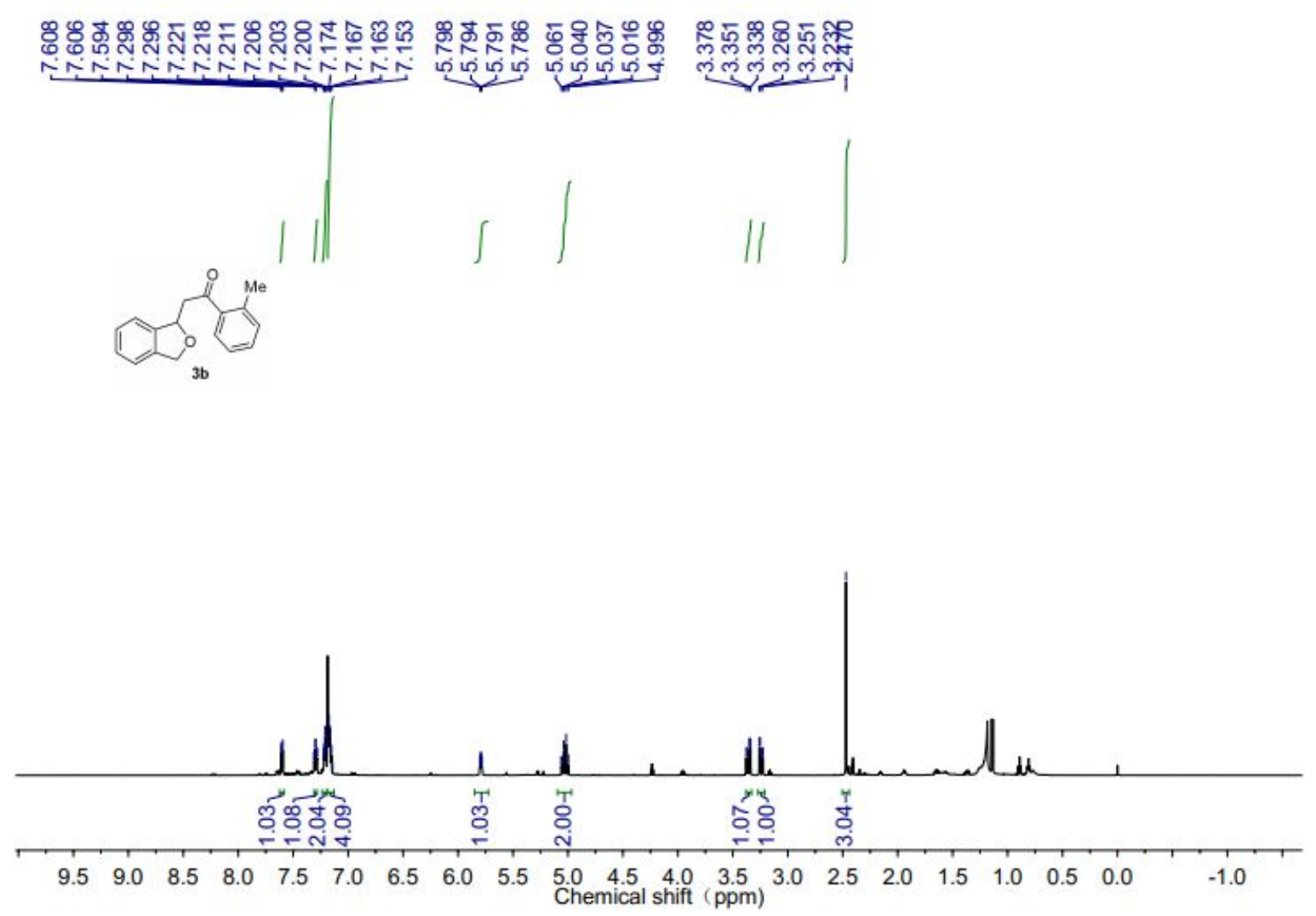

${ }^{1} \mathbf{H}$ NMR (600 MHz, $\left.\mathrm{CDCl}_{3}, 298 \mathrm{~K}\right)$ of $\mathbf{3 b}$

\begin{tabular}{|c|c|c|c|}
\hline $\begin{array}{l}\text { R } \\
\Sigma\end{array}$ & 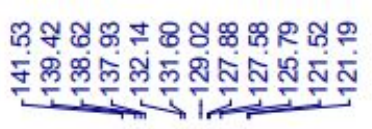 & & $\begin{array}{l}\text { y } \\
\text { of } \\
\end{array}$ \\
\hline
\end{tabular}
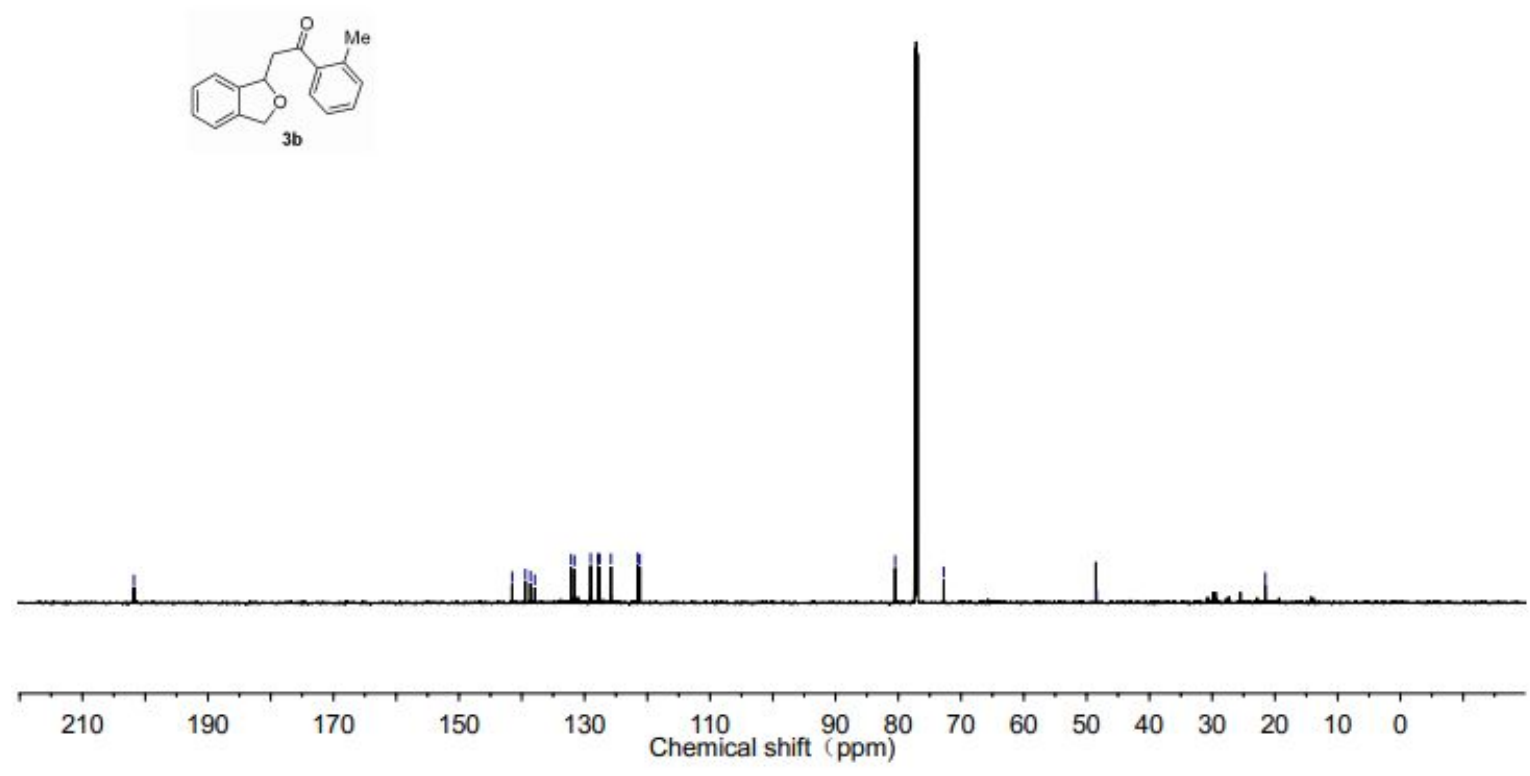

${ }^{13} \mathbf{C}\left\{{ }^{1} \mathbf{H}\right\}$ NMR $\left(151 \mathrm{MHz}, \mathrm{CDCl}_{3}, 298 \mathrm{~K}\right)$ of $\mathbf{3 b}$ 


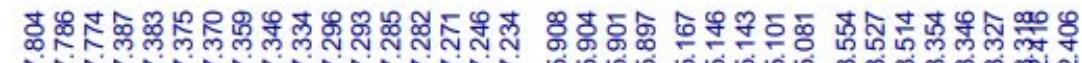

||$_{\mid}||_{\mid} \mid$

$\mathrm{C}_{3 \mathrm{C}}^{\mathrm{Me}}$

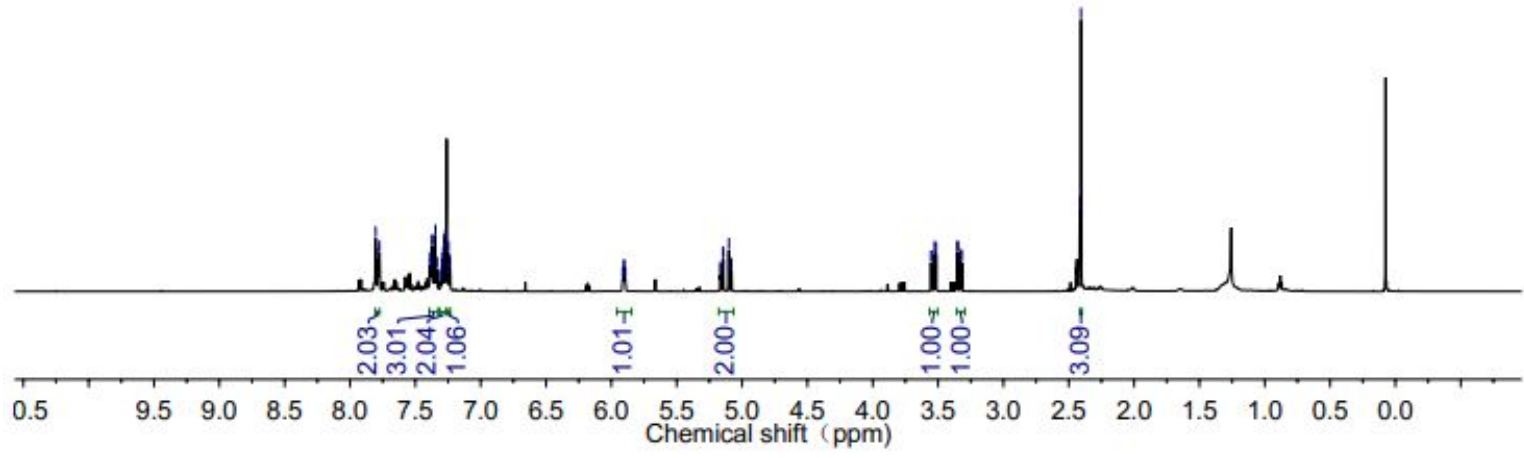

${ }^{1} \mathbf{H}$ NMR $\left(600 \mathrm{MHz}, \mathrm{CDCl}_{3}, 298 \mathrm{~K}\right)$ of $\mathbf{3 c}$

\begin{tabular}{|c|c|c|c|}
\hline कृ & 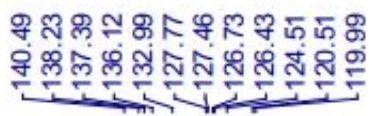 & 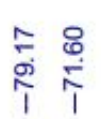 & $\begin{array}{c}\hat{0} \\
\stackrel{j}{j}\end{array}$ \\
\hline
\end{tabular}
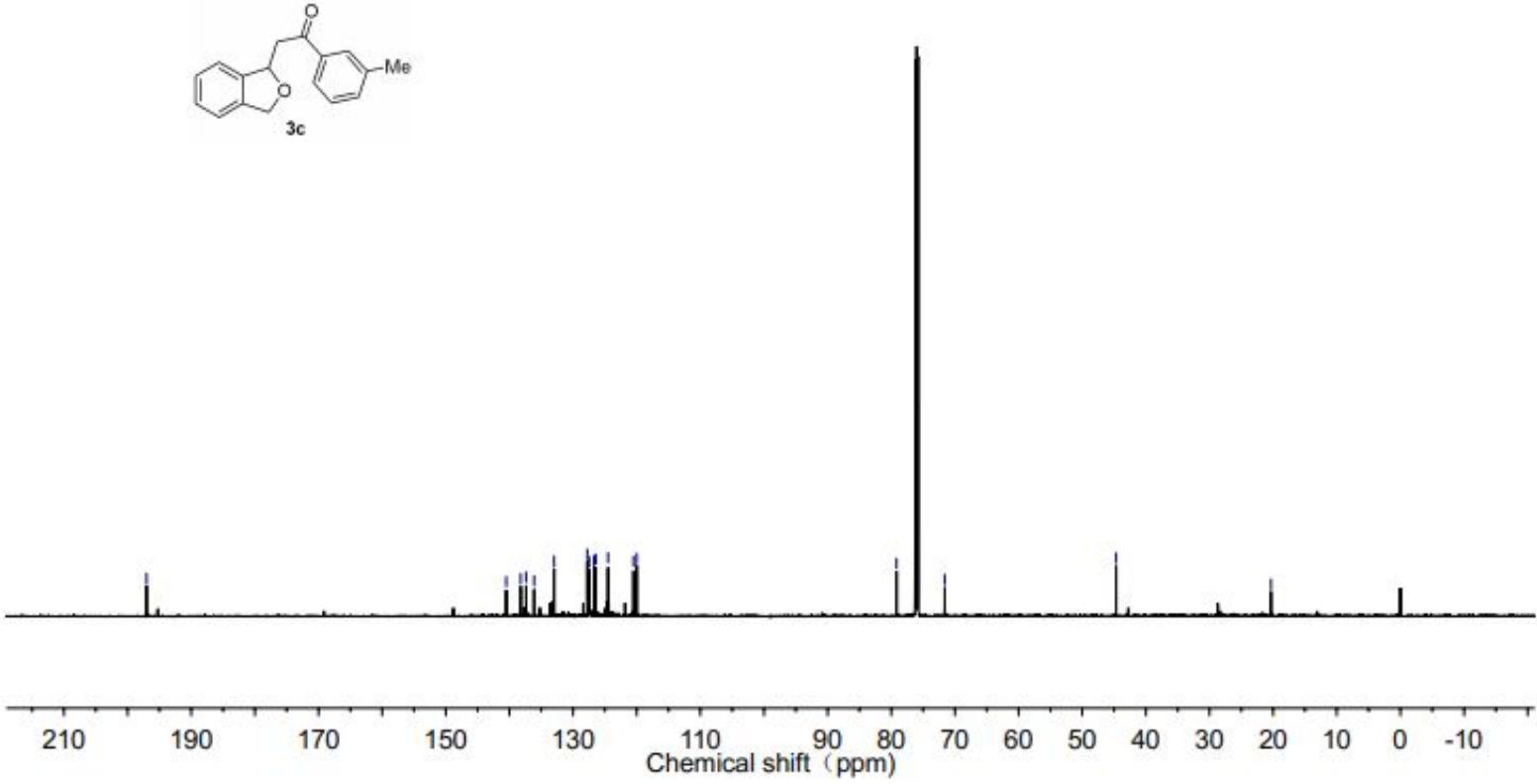

${ }^{13} \mathbf{C}\left\{{ }^{1} \mathbf{H}\right\}$ NMR $\left(151 \mathrm{MHz}, \mathrm{CDCl}_{3}, 298 \mathrm{~K}\right)$ of $\mathbf{3 c}$ 


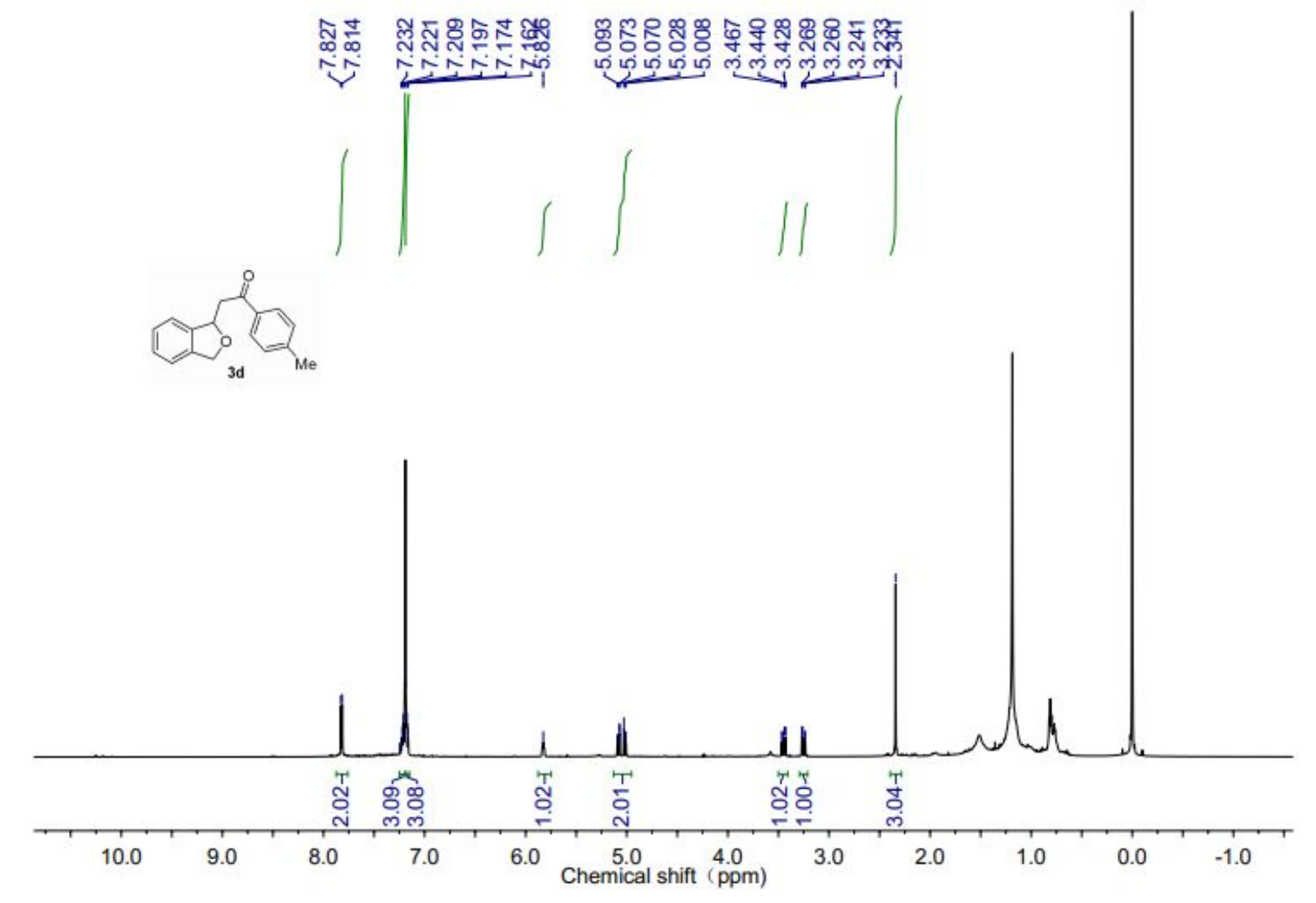

${ }^{1} \mathbf{H}$ NMR (600 MHz, $\left.\mathrm{CDCl}_{3}, 298 \mathrm{~K}\right)$ of $\mathbf{3 d}$

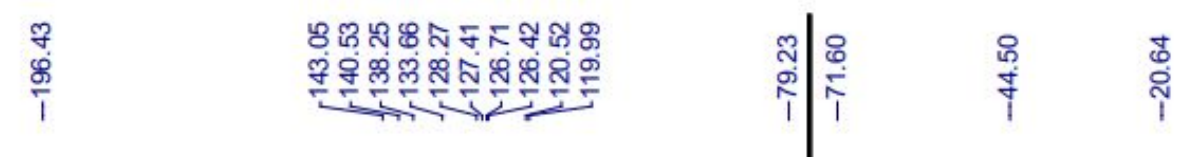
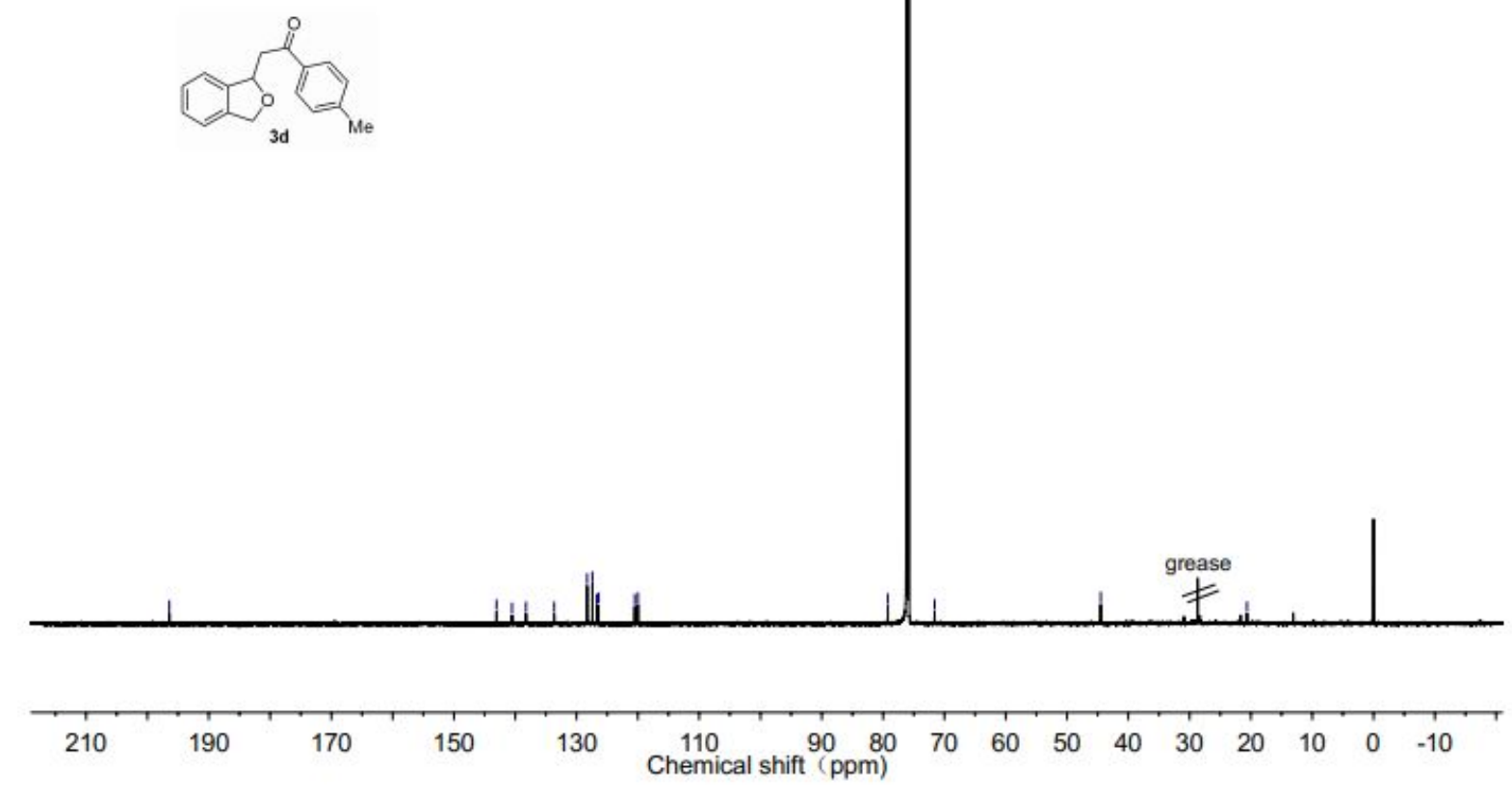

${ }^{13} \mathbf{C}\left\{{ }^{1} \mathbf{H}\right\}$ NMR $\left(151 \mathrm{MHz}, \mathrm{CDCl}_{3}, 298 \mathrm{~K}\right)$ of $\mathbf{3 d}$ 

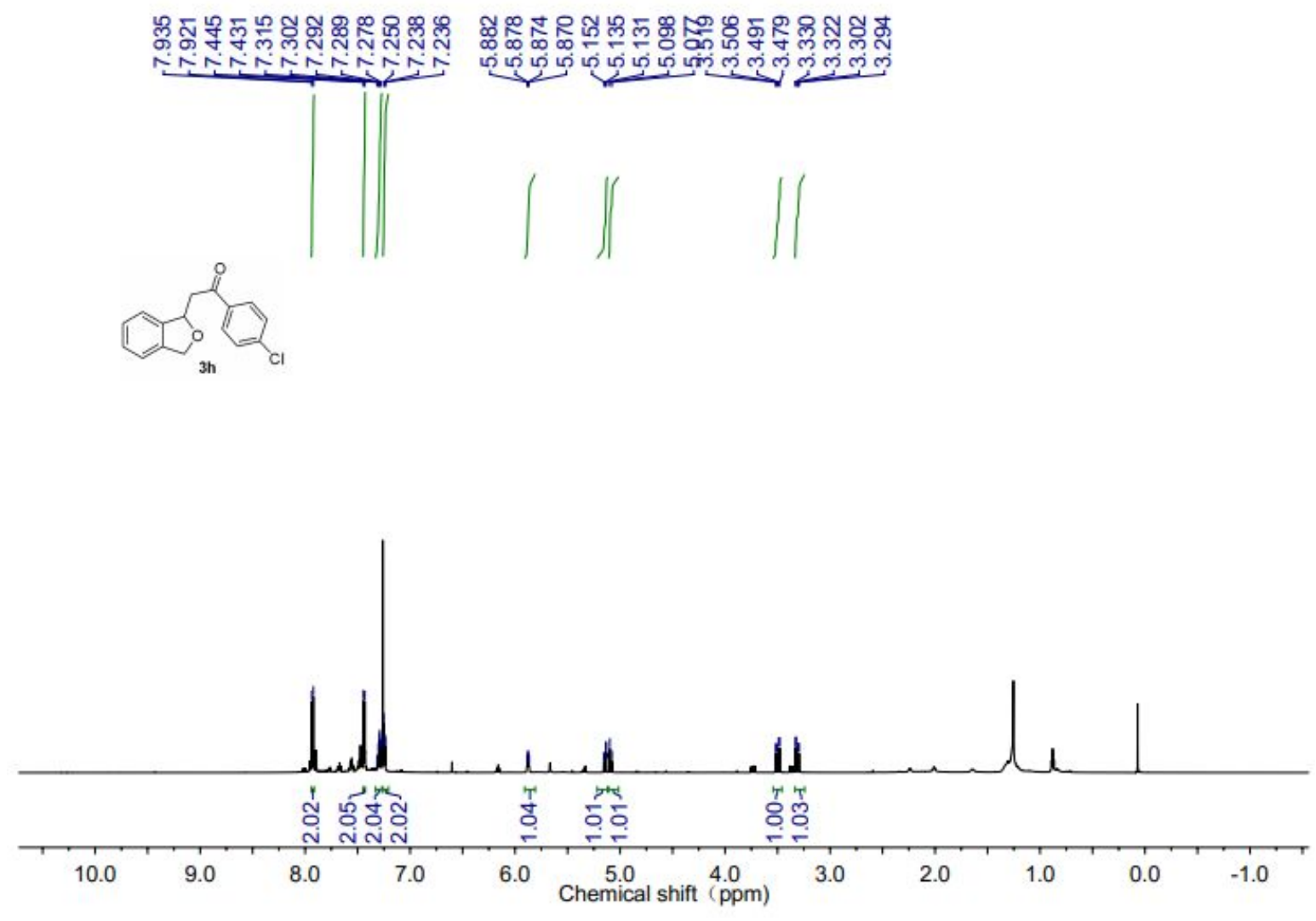

${ }^{1} \mathbf{H}$ NMR (600 MHz, $\left.\mathrm{CDCl}_{3}, 298 \mathrm{~K}\right)$ of $\mathbf{3 h}$
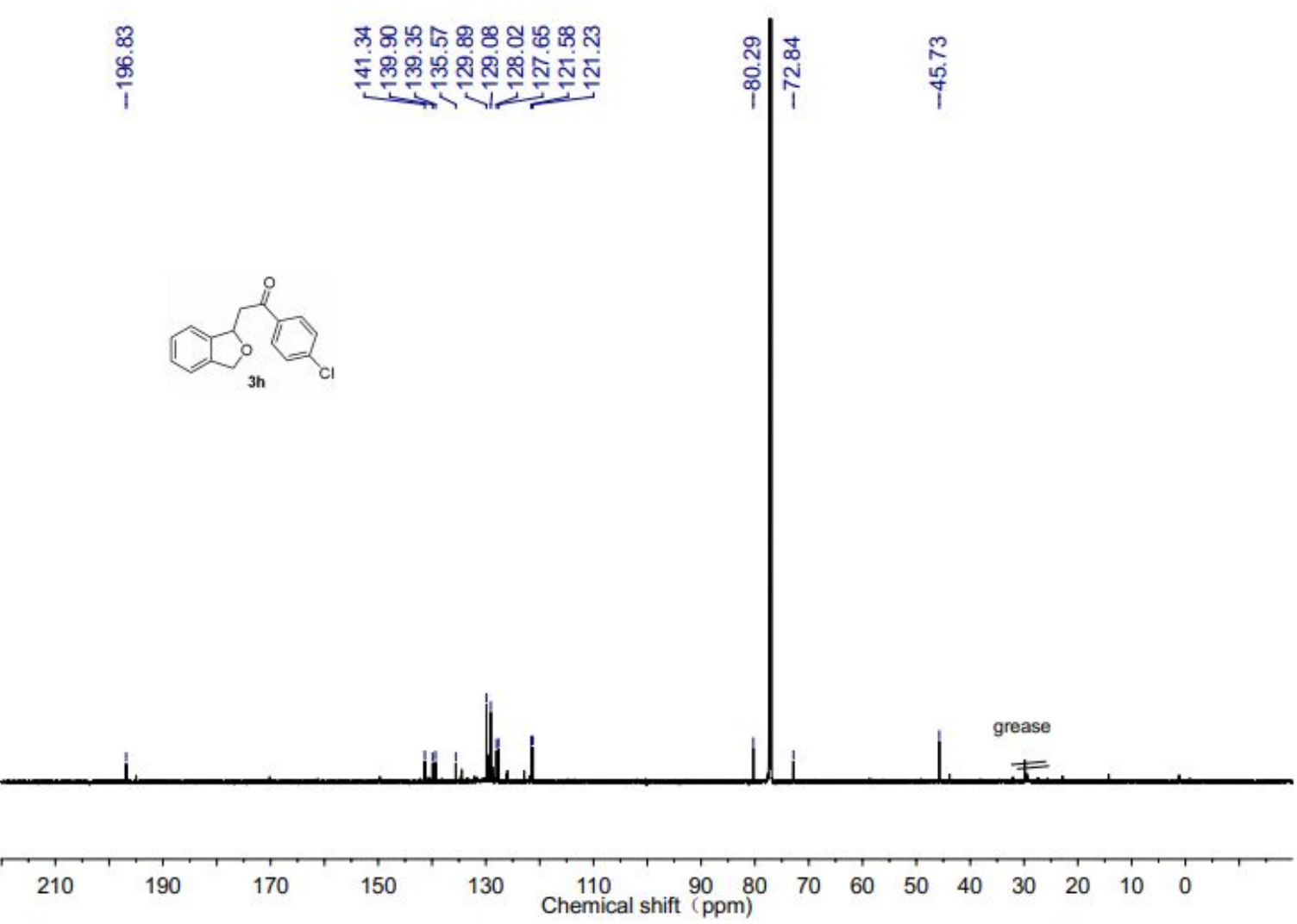

${ }^{13} \mathbf{C}\left\{{ }^{1} \mathbf{H}\right\}$ NMR $\left(151 \mathrm{MHz}, \mathrm{CDCl}_{3}, 298 \mathrm{~K}\right)$ of $\mathbf{3 h}$ 


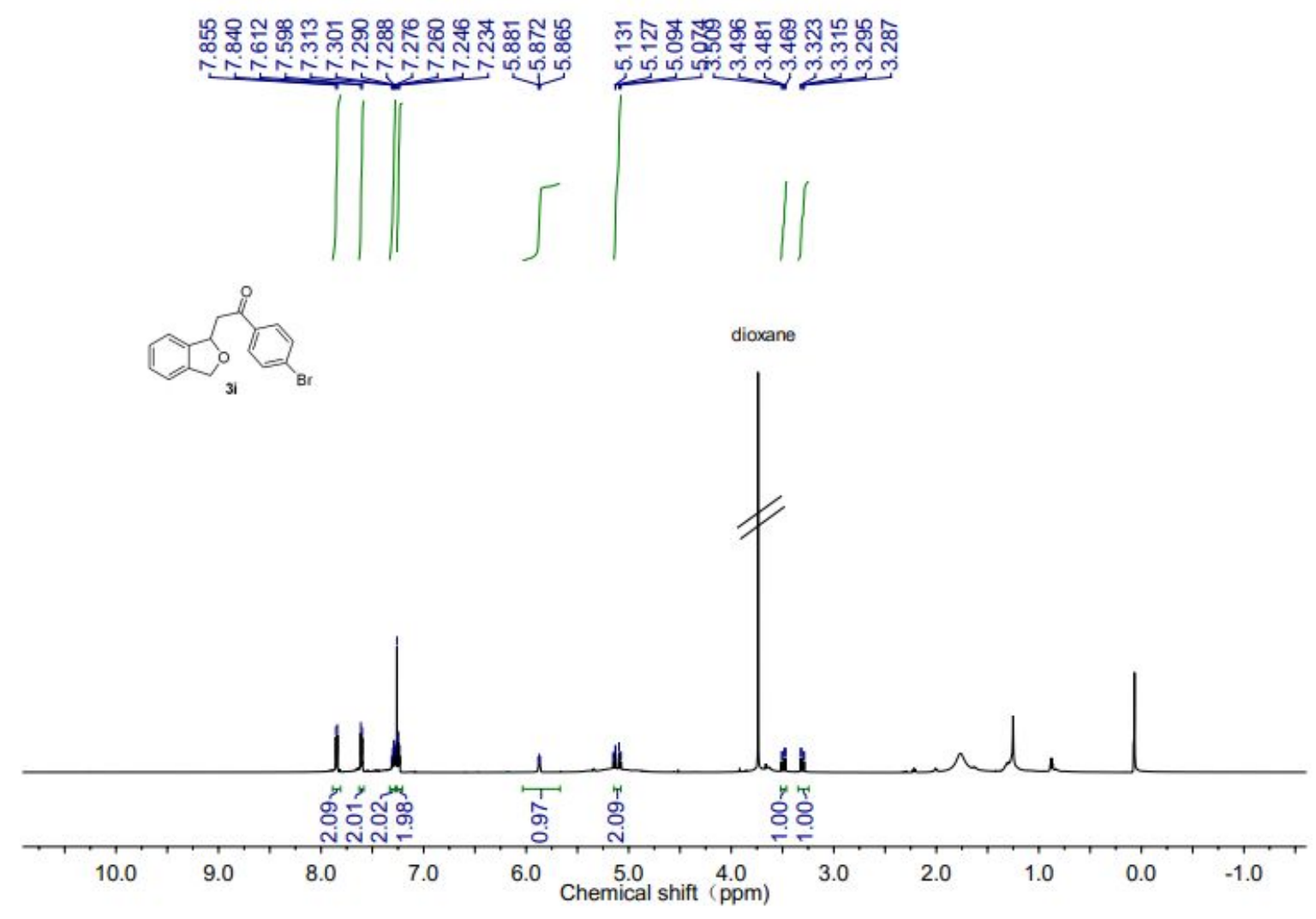

${ }^{1} \mathbf{H}$ NMR $\left(600 \mathrm{MHz}, \mathrm{CDCl}_{3}, 298 \mathrm{~K}\right)$ of $\mathbf{3 i}$
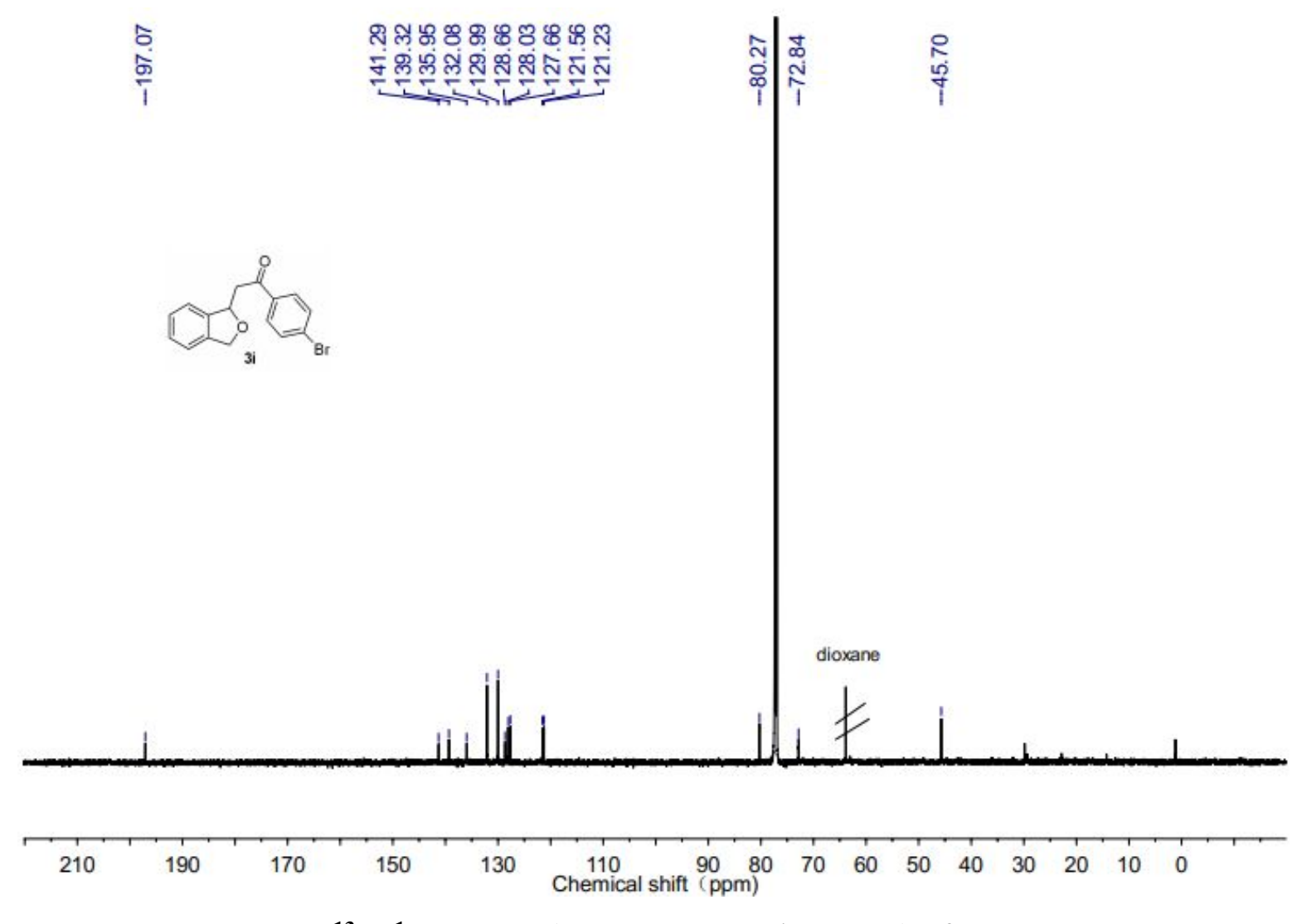

${ }^{13} \mathbf{C}\left\{{ }^{1} \mathbf{H}\right\}$ NMR $\left(151 \mathrm{MHz}, \mathrm{CDCl}_{3}, 298 \mathrm{~K}\right)$ of $\mathbf{3 i}$ 


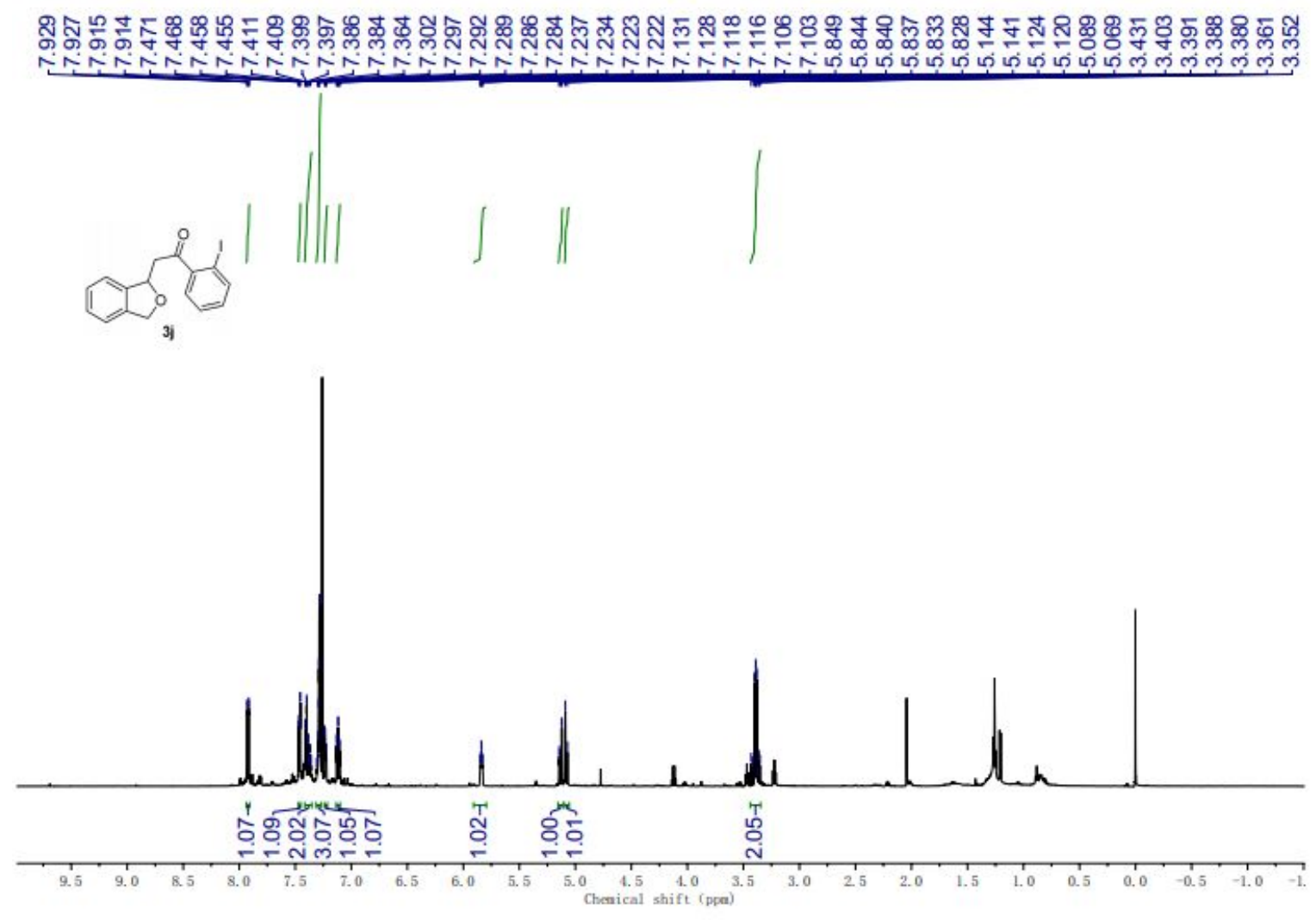

${ }^{1} \mathbf{H}$ NMR $\left(600 \mathrm{MHz}, \mathrm{CDCl}_{3}, 298 \mathrm{~K}\right)$ of $\mathbf{3 j}$
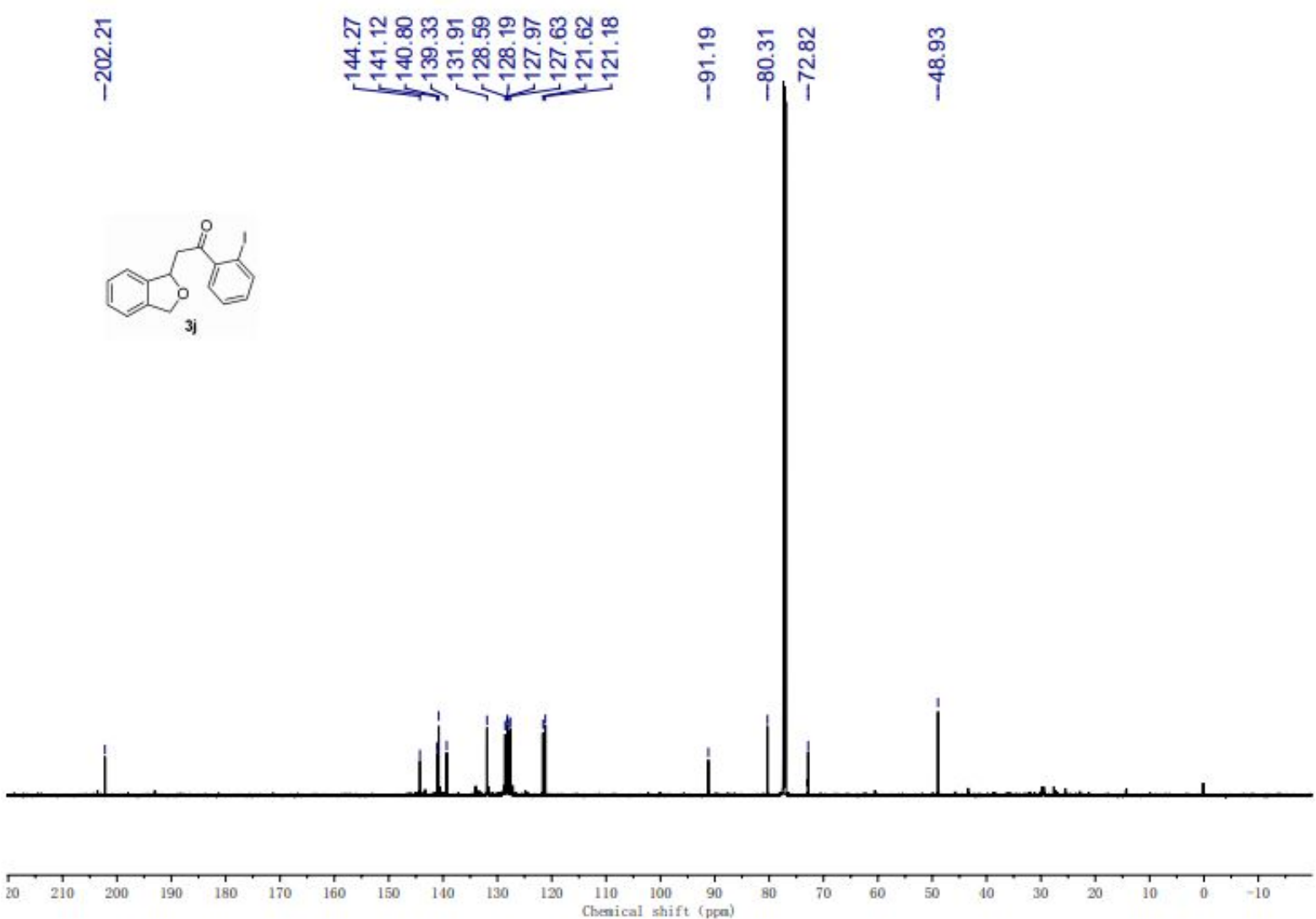

${ }^{13} \mathbf{C}\left\{{ }^{1} \mathbf{H}\right\}$ NMR $\left(151 \mathrm{MHz}, \mathrm{CDCl}_{3}, 298 \mathrm{~K}\right)$ of $\mathbf{3 j}$ 

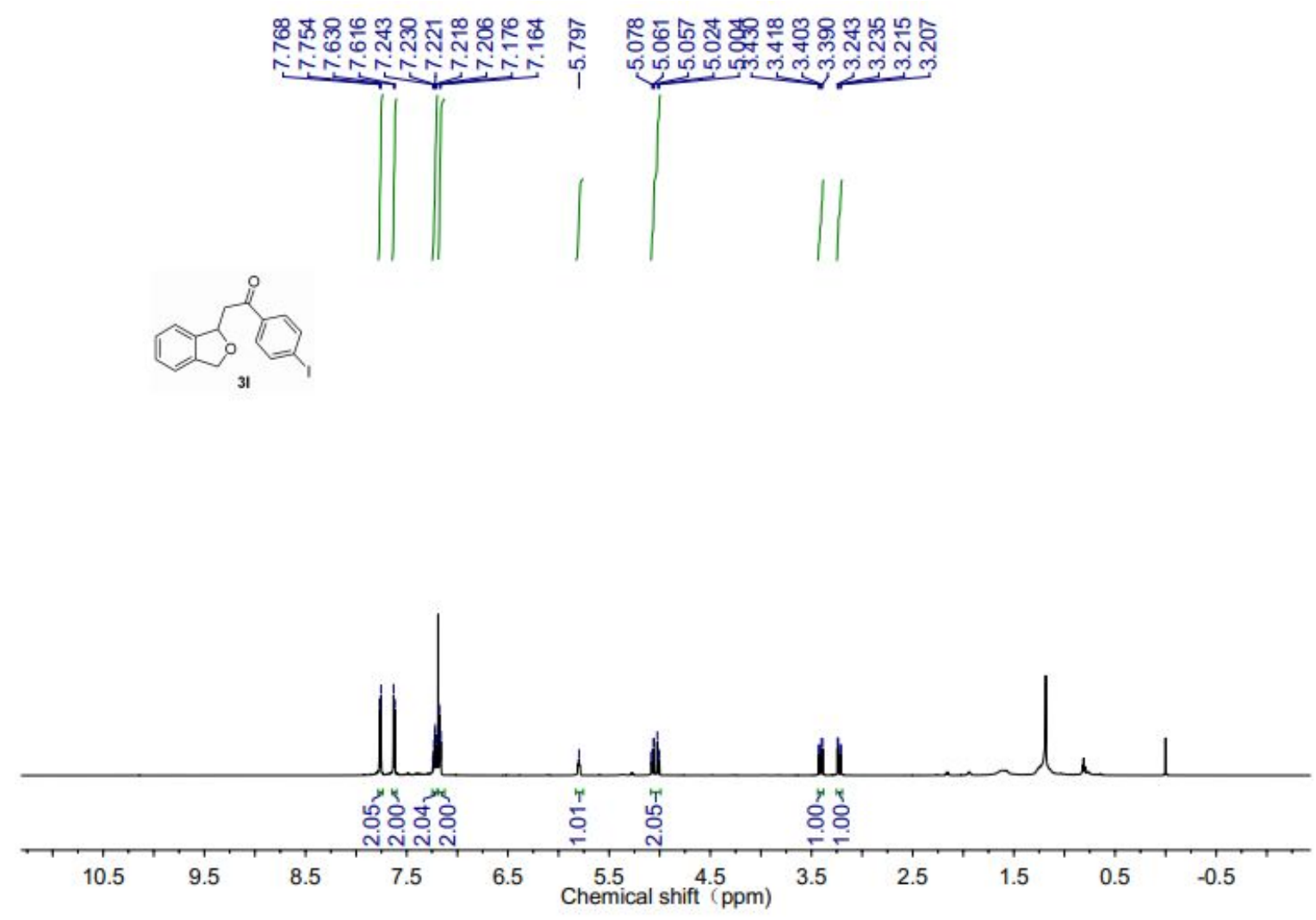

${ }^{1} \mathbf{H}$ NMR $\left(600 \mathrm{MHz}, \mathrm{CDCl}_{3}, 298 \mathrm{~K}\right)$ of $3 \mathbf{I}$
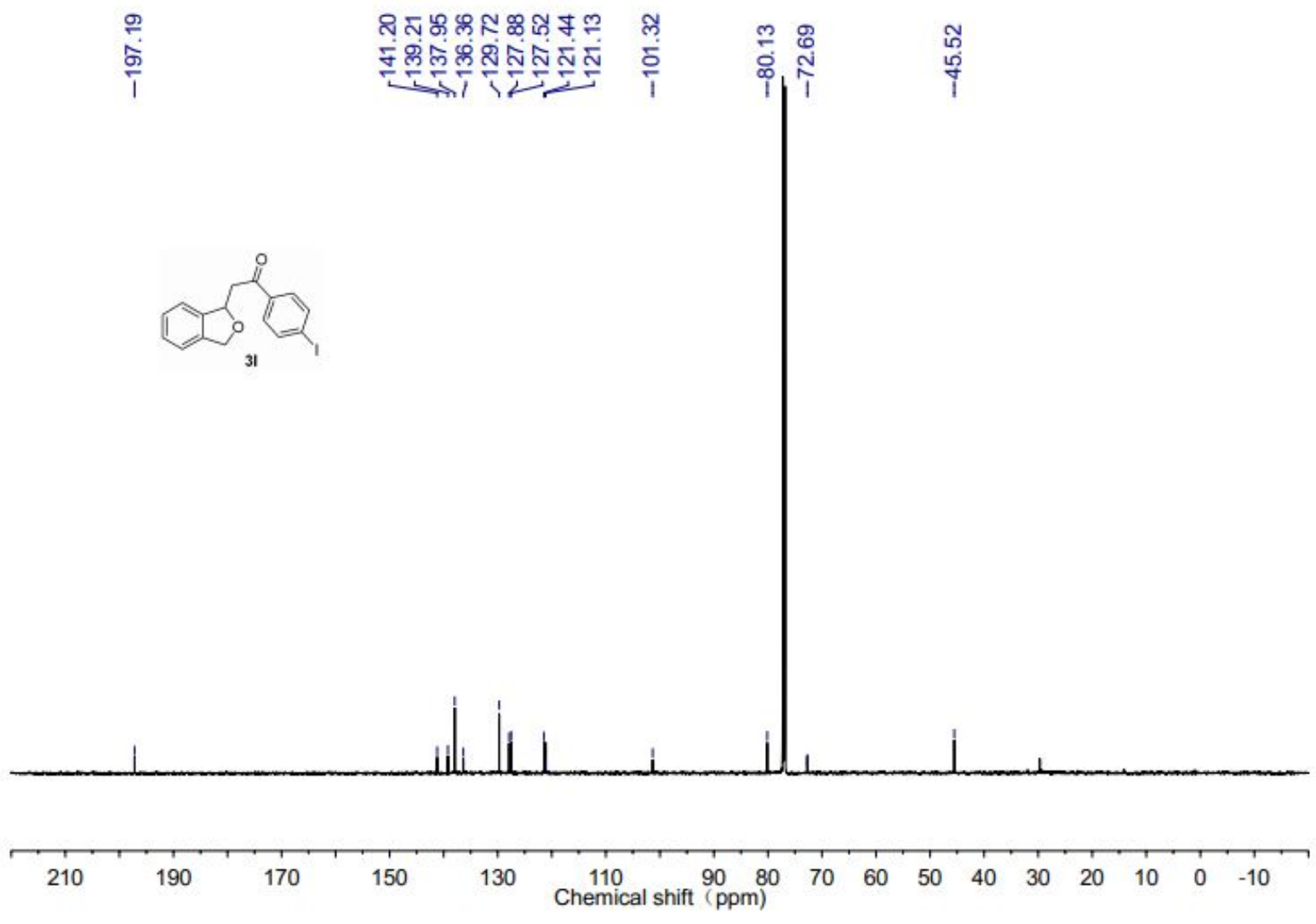

${ }^{\mathbf{1 3}} \mathbf{C}\left\{{ }^{\mathbf{H}} \mathrm{H}\right\} \mathbf{N M R}\left(151 \mathrm{MHz}, \mathrm{CDCl}_{3}, 298 \mathrm{~K}\right)$ of $\mathbf{3 l}$ 

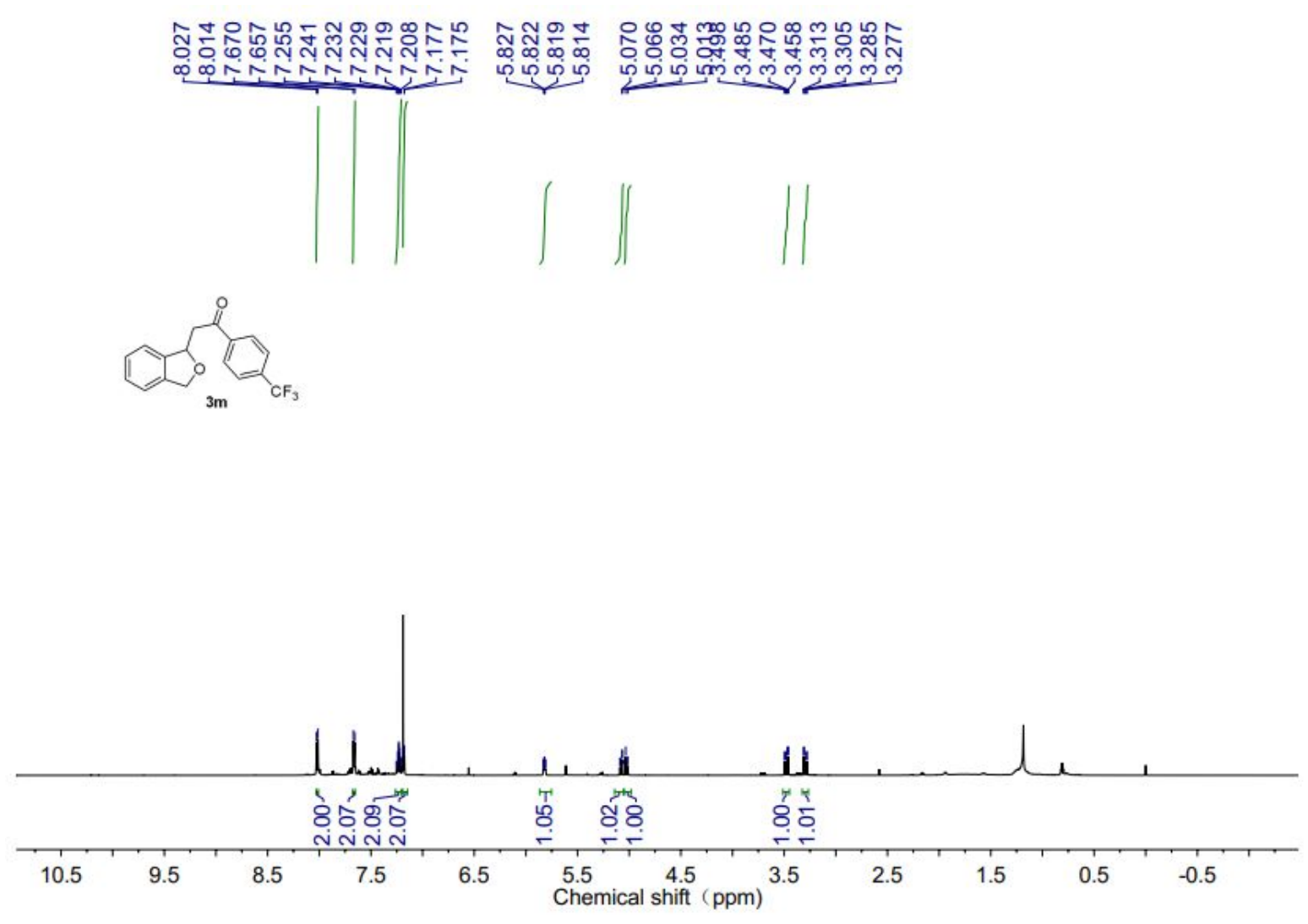

${ }^{1} \mathbf{H}$ NMR $\left(600 \mathrm{MHz}, \mathrm{CDCl}_{3}, 298 \mathrm{~K}\right)$ of $\mathbf{3 m}$
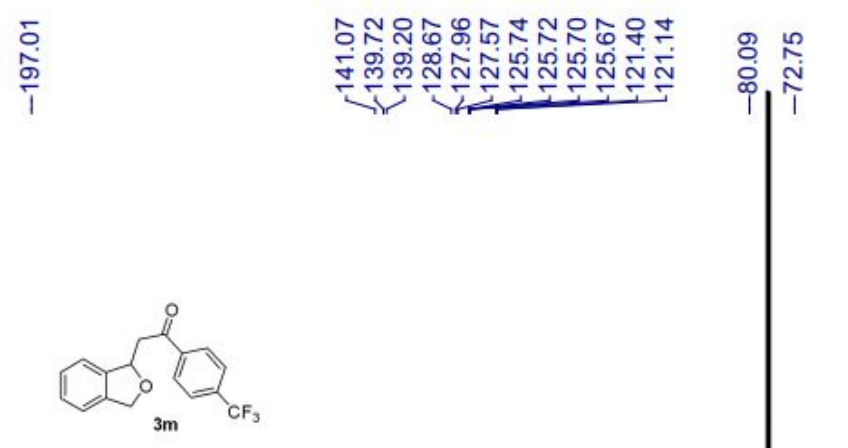

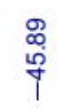
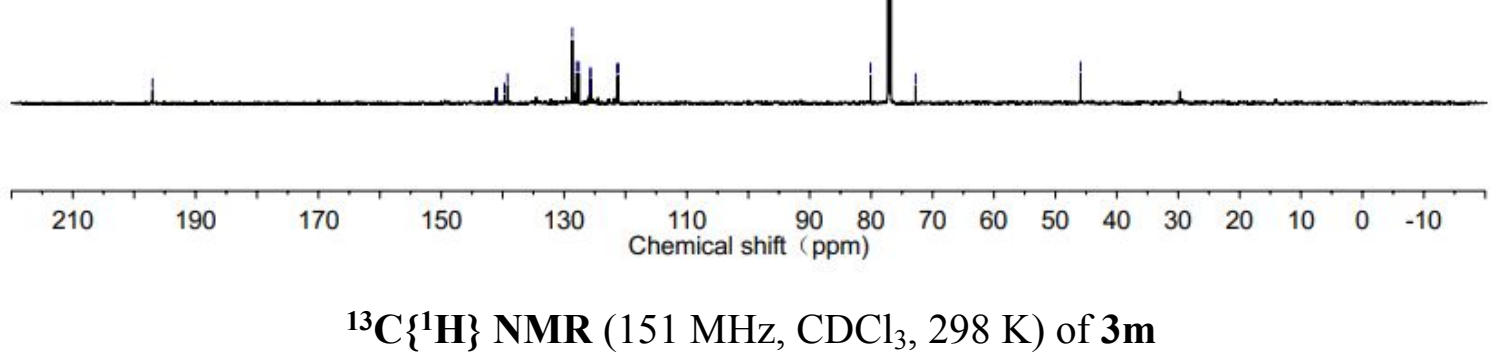

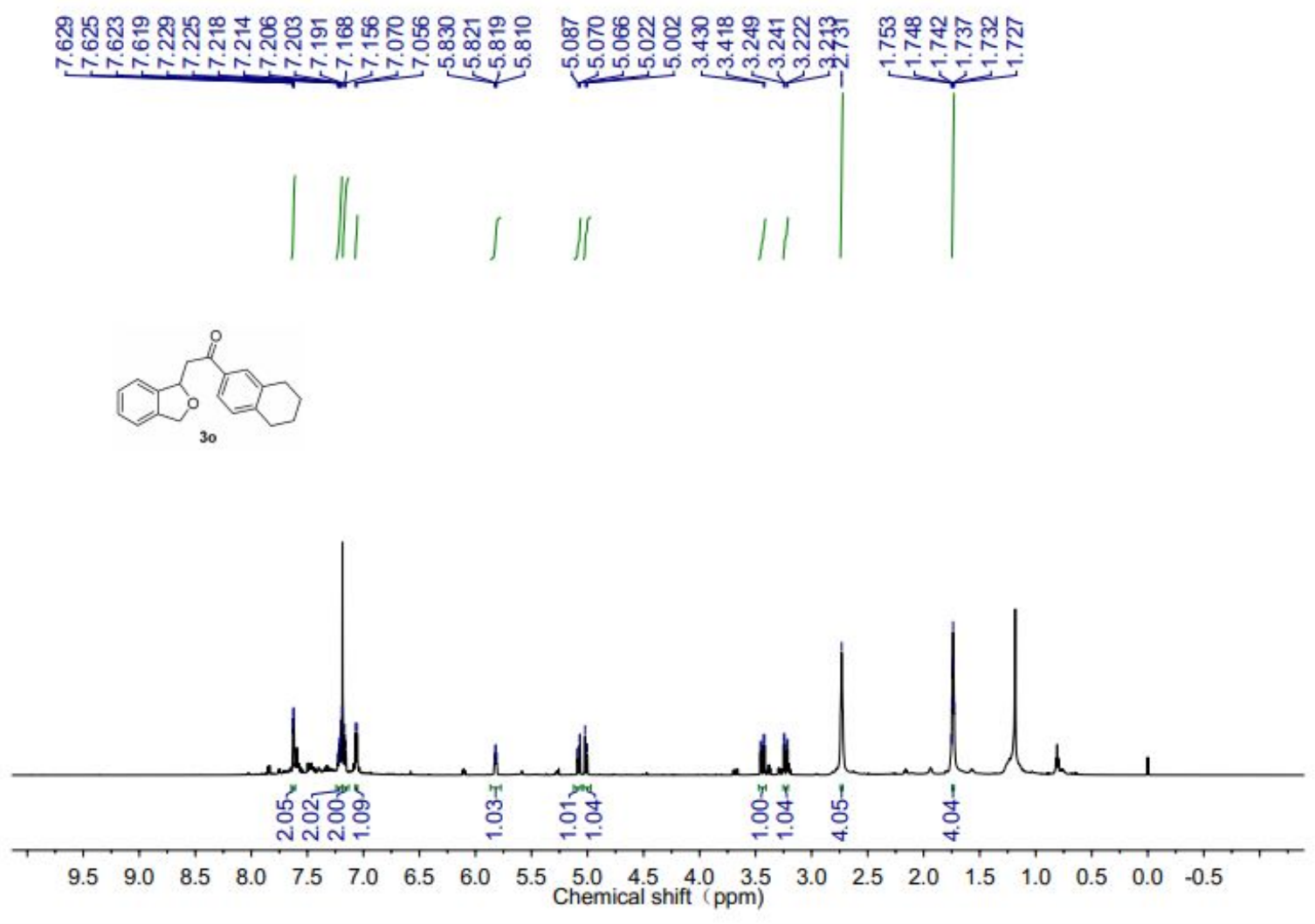

${ }^{1} \mathbf{H}$ NMR $\left(600 \mathrm{MHz}, \mathrm{CDCl}_{3}, 298 \mathrm{~K}\right)$ of $\mathbf{3 o}$

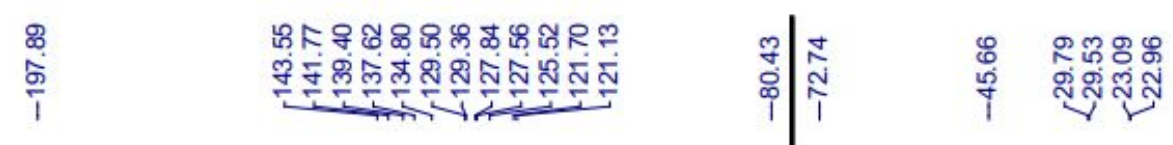

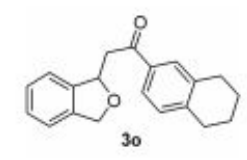

ألفاللفينتننا

\begin{tabular}{|c|c|c|c|c|c|c|c|c|c|c|c|c|c|}
\hline 210 & 190 & 170 & 150 & 130 & $\begin{array}{c}110 \\
\text { Chemical shift }\end{array} \stackrel{90}{(\mathrm{ppm})} 80$ & 70 & 60 & 50 & 40 & 30 & 20 & 10 & 0 \\
\hline
\end{tabular}




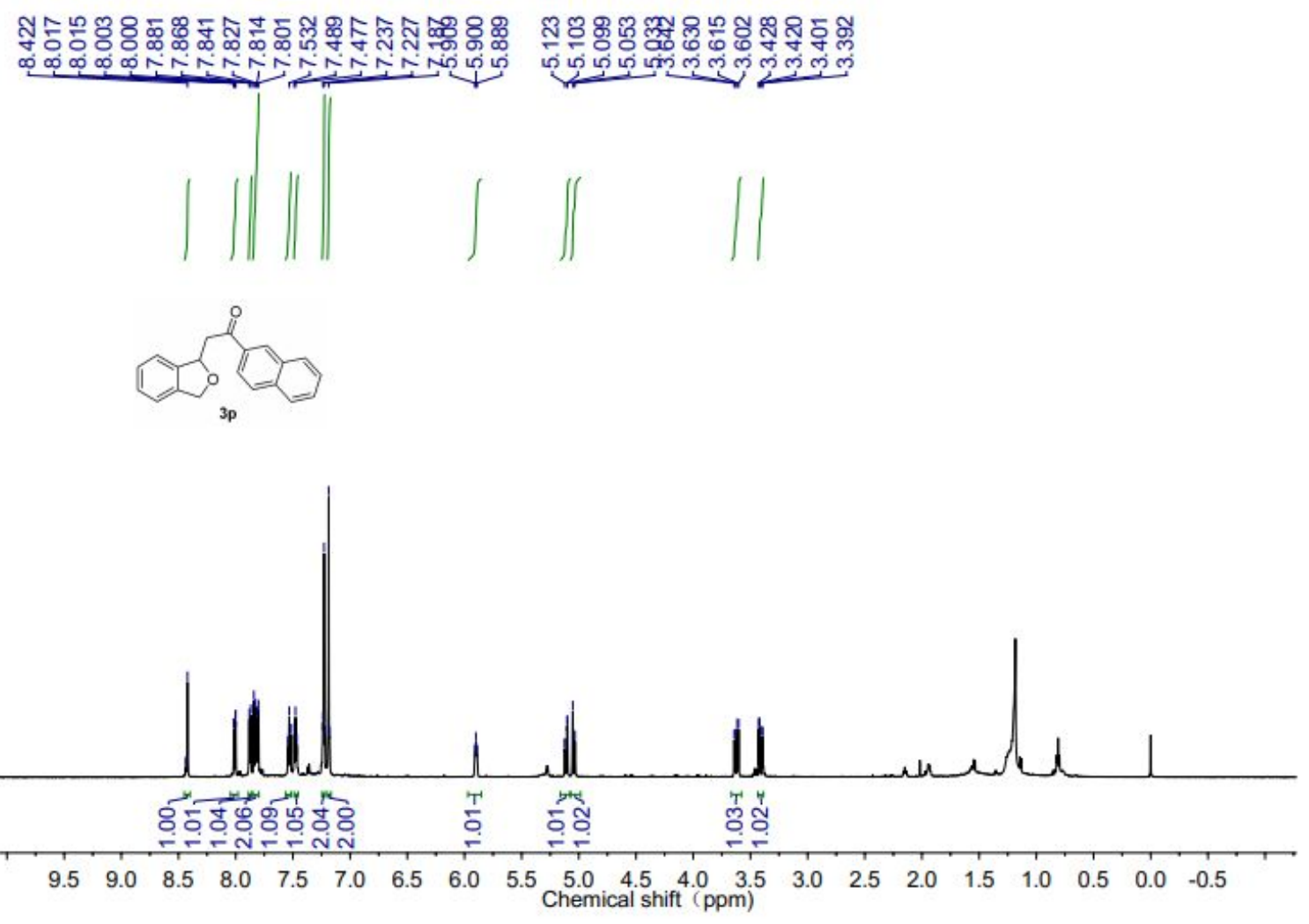

${ }^{1} \mathbf{H}$ NMR $\left(600 \mathrm{MHz}, \mathrm{CDCl}_{3}, 298 \mathrm{~K}\right)$ of $\mathbf{3 p}$

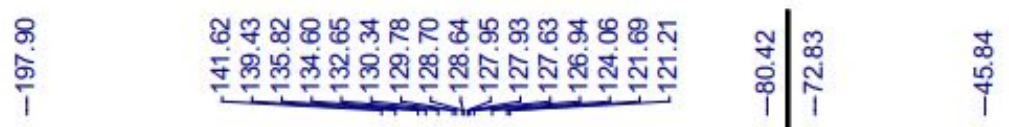
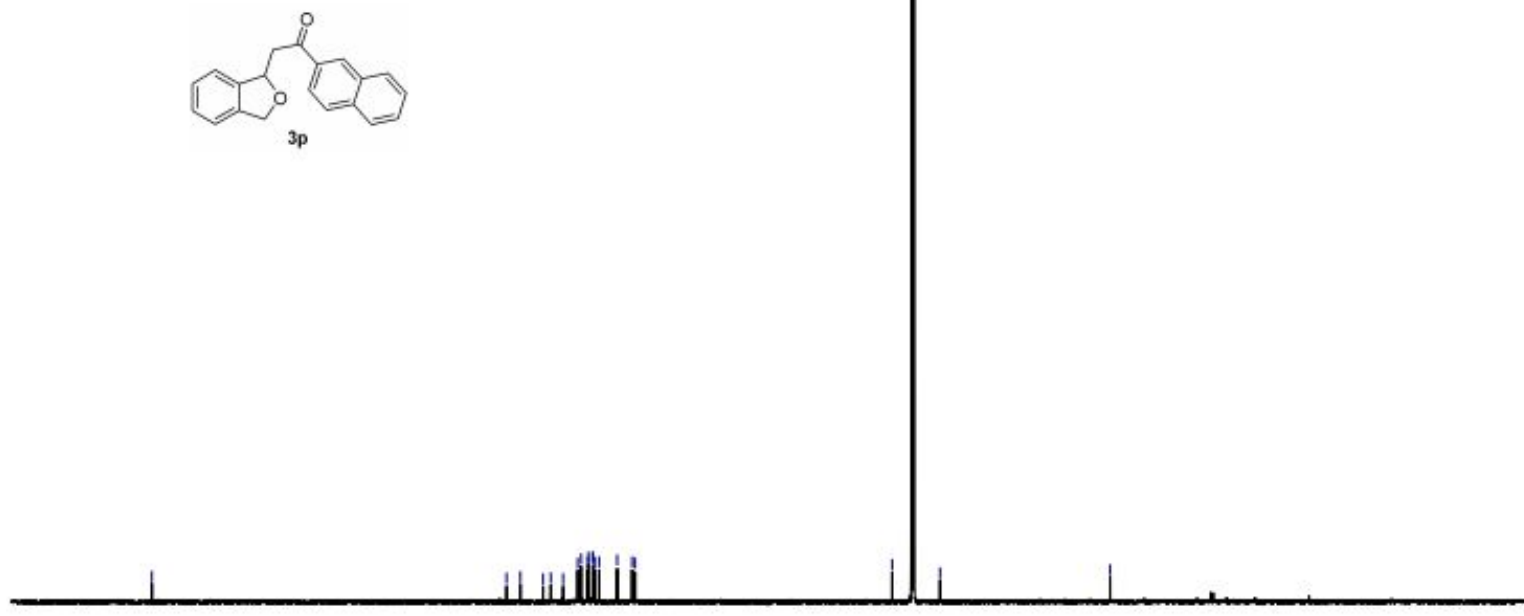

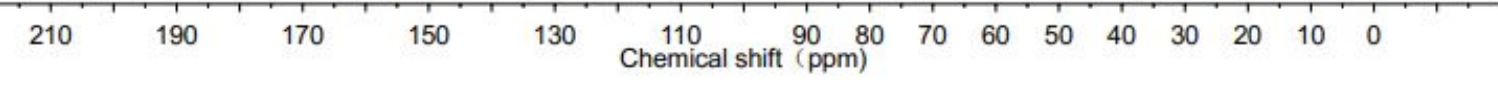

${ }^{13} \mathbf{C}\left\{{ }^{1} \mathbf{H}\right\} \mathbf{N M R}\left(151 \mathrm{MHz}, \mathrm{CDCl}_{3}, 298 \mathrm{~K}\right)$ of $\mathbf{3 p}$ 


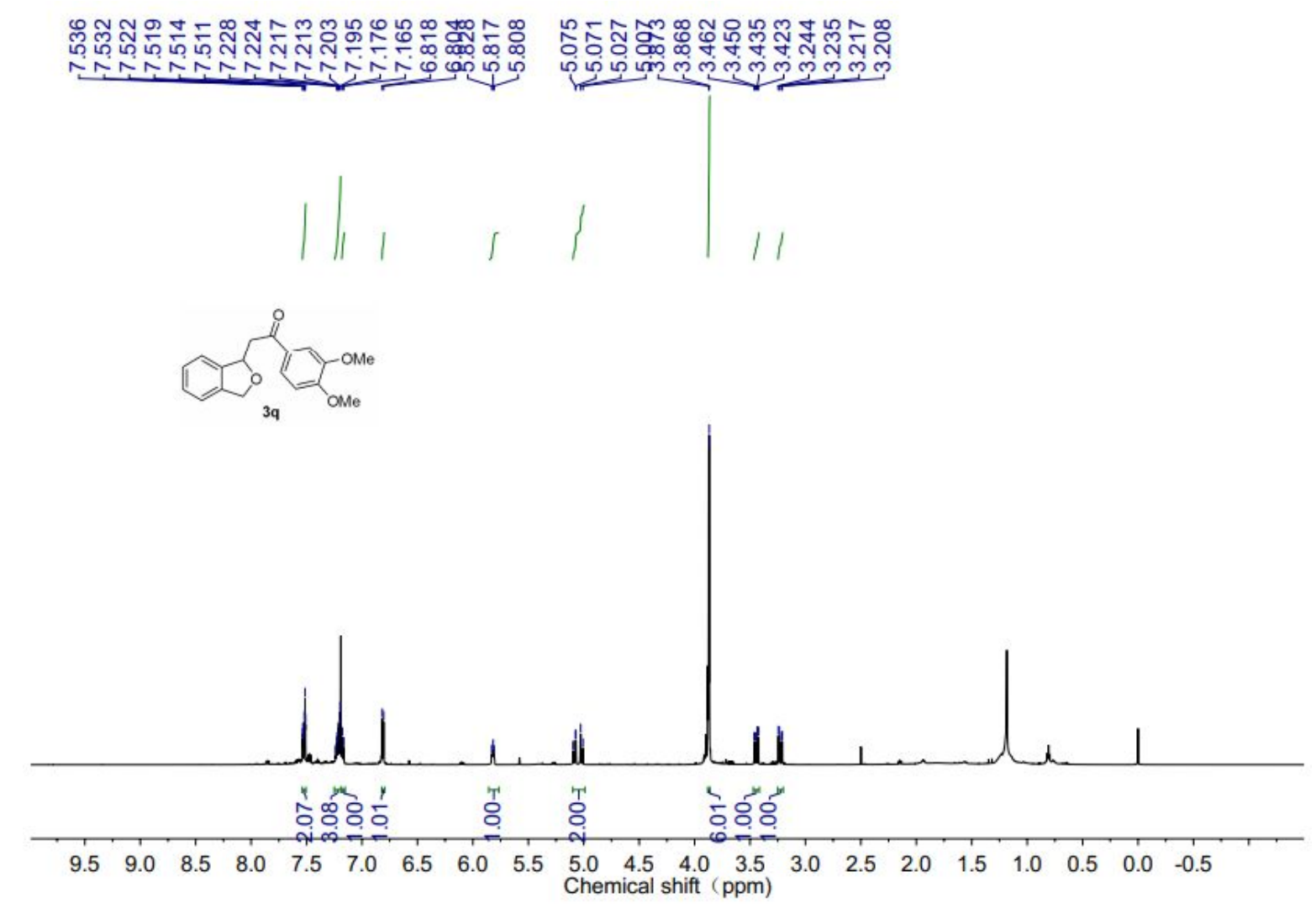

${ }^{1} \mathbf{H}$ NMR (600 MHz, $\left.\mathrm{CDCl}_{3}, 298 \mathrm{~K}\right)$ of $\mathbf{3 q}$

\begin{tabular}{l} 
क्ल \\
ळ \\
\hline
\end{tabular}

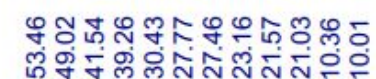
กำ

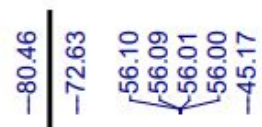
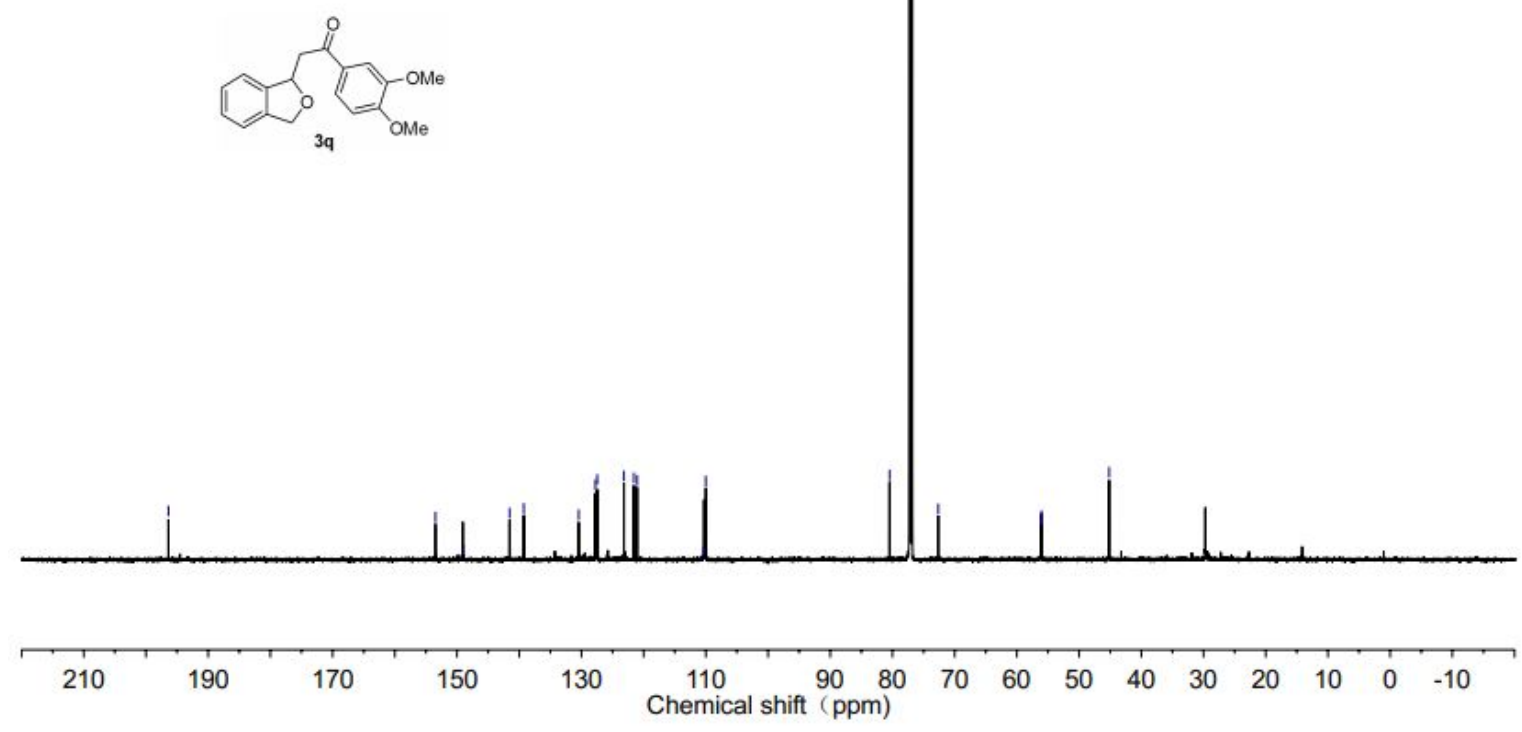

${ }^{13} \mathbf{C}\left\{{ }^{1} \mathbf{H}\right\}$ NMR $\left(151 \mathrm{MHz}, \mathrm{CDCl}_{3}, 298 \mathrm{~K}\right)$ of $\mathbf{3 q}$ 


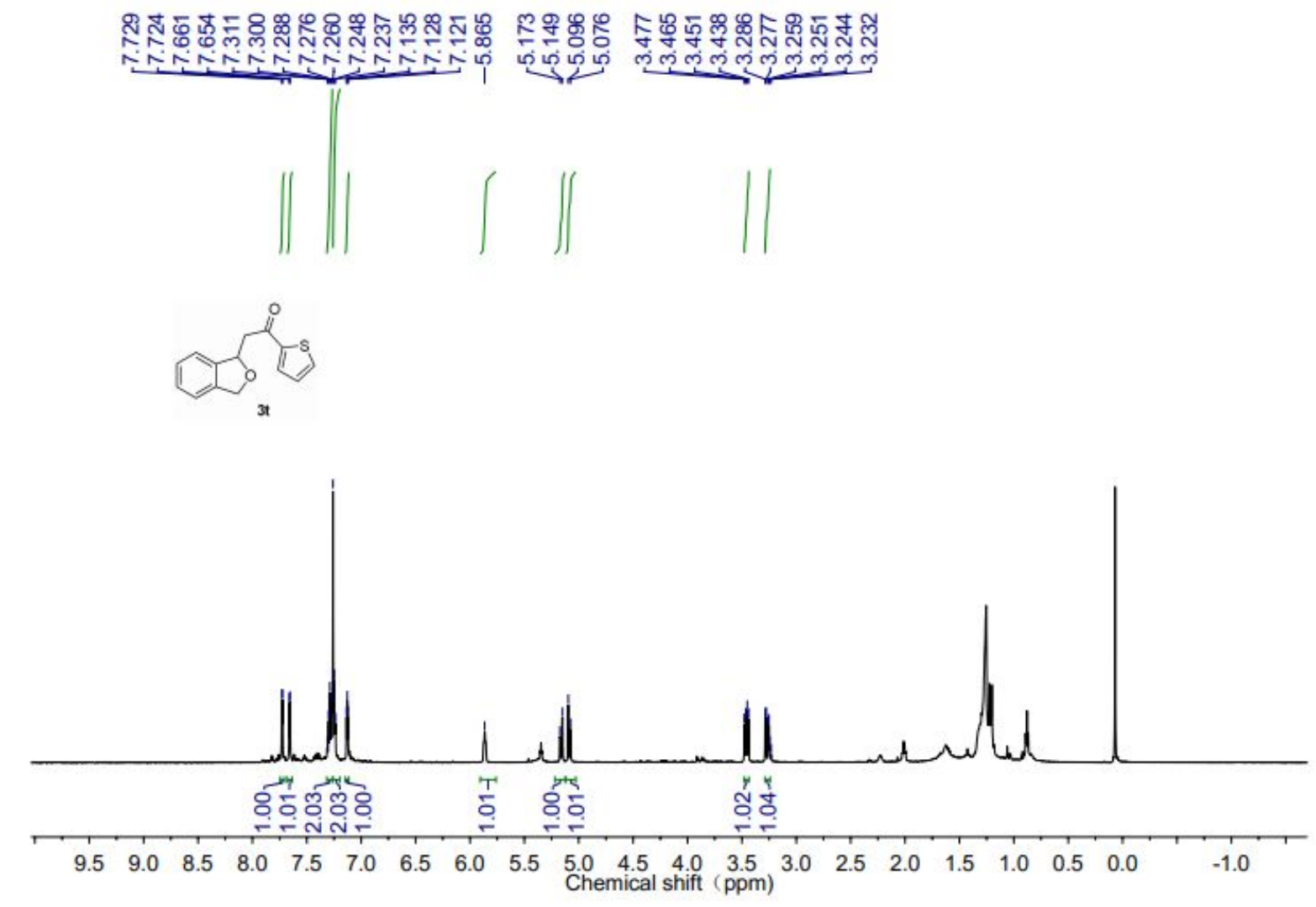

${ }^{1} \mathbf{H}$ NMR (600 MHz, $\left.\mathrm{CDCl}_{3}, 298 \mathrm{~K}\right)$ of $\mathbf{3 t}$

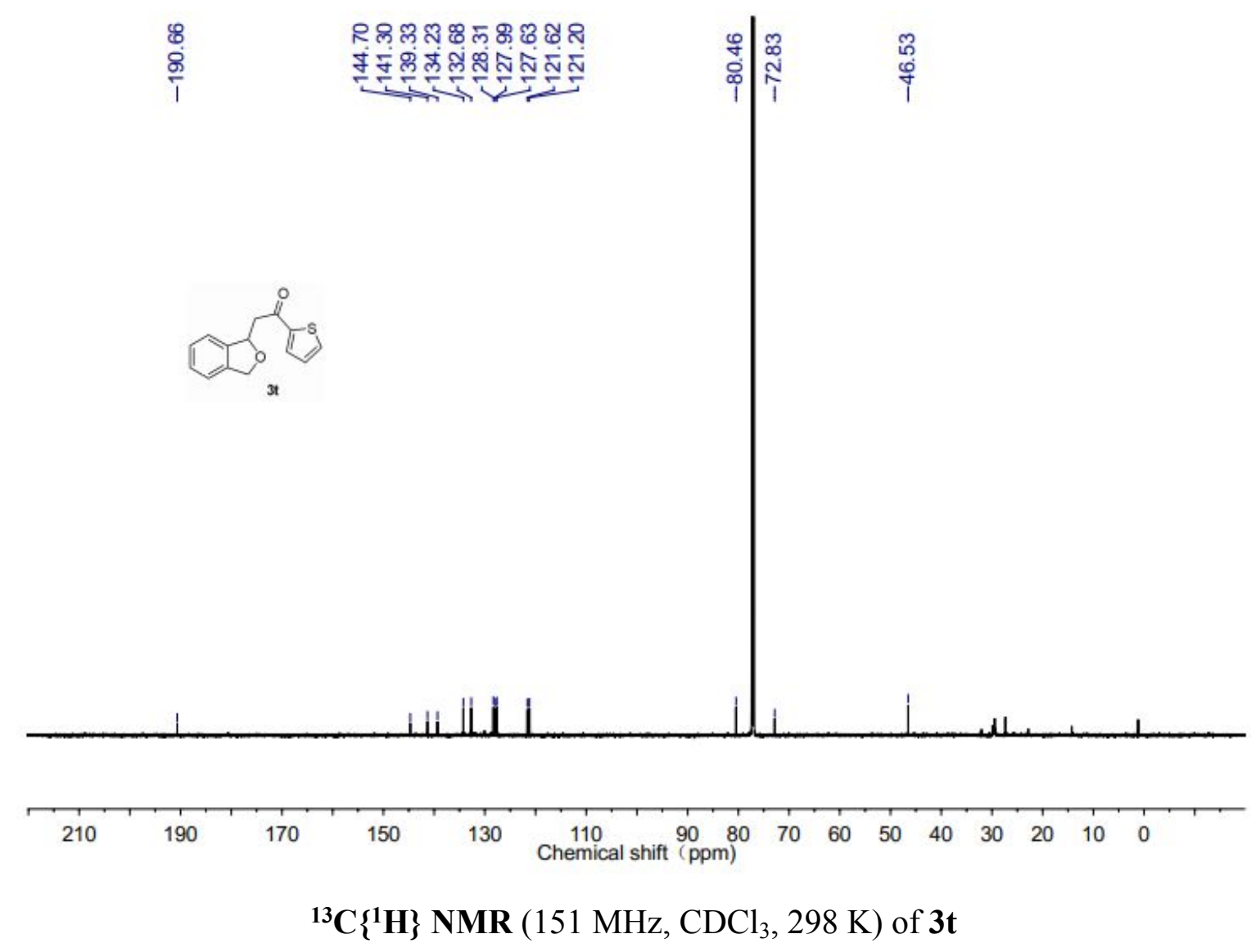




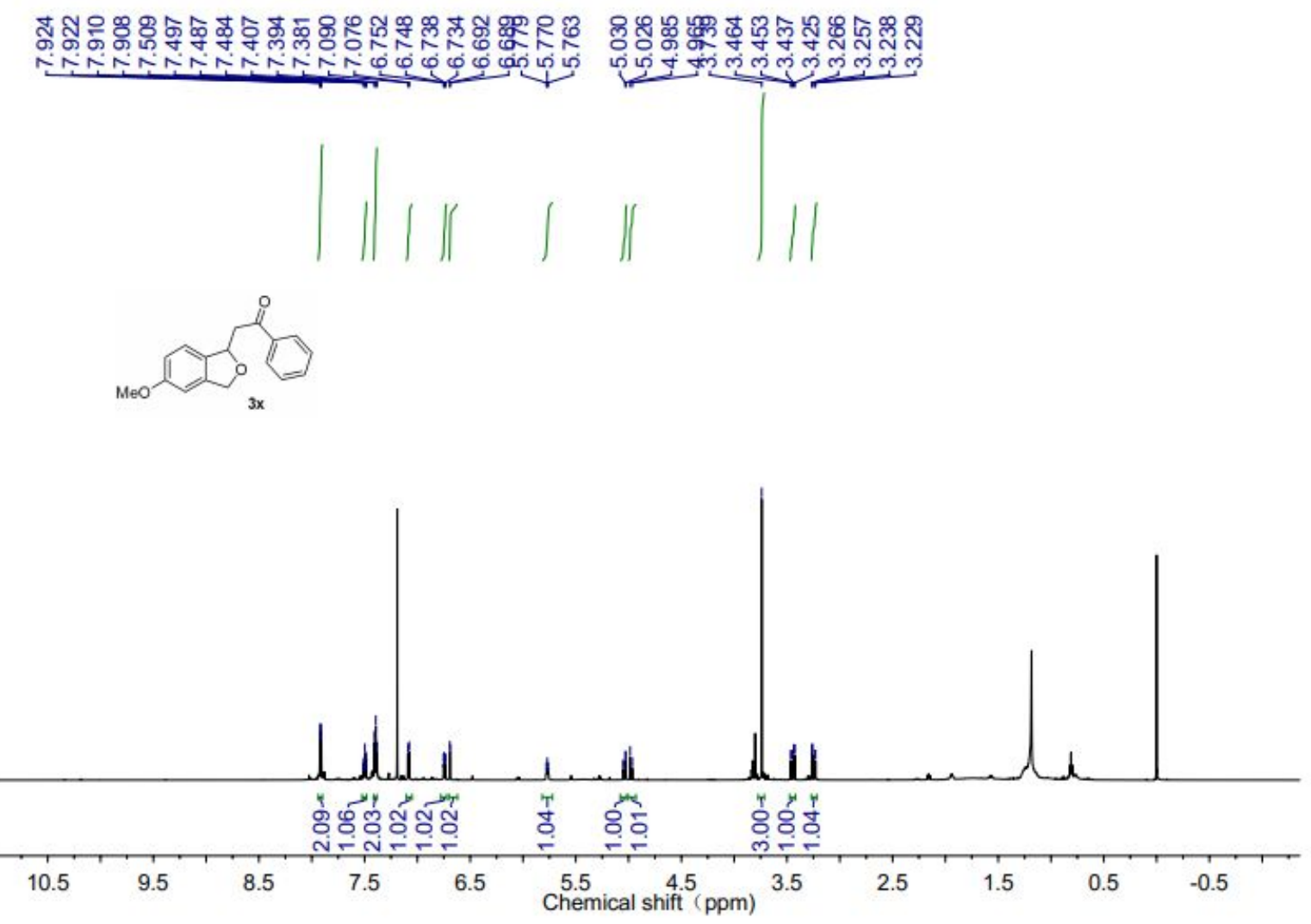

${ }^{1} \mathbf{H}$ NMR $\left(600 \mathrm{MHz}, \mathrm{CDCl}_{3}, 298 \mathrm{~K}\right)$ of $\mathbf{3 x}$

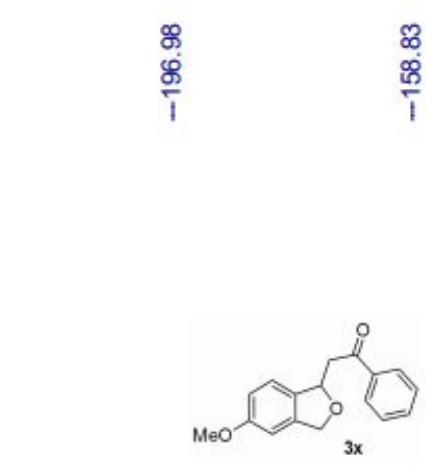

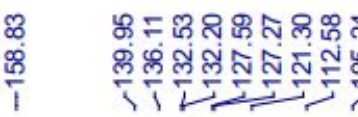

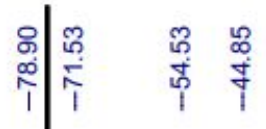
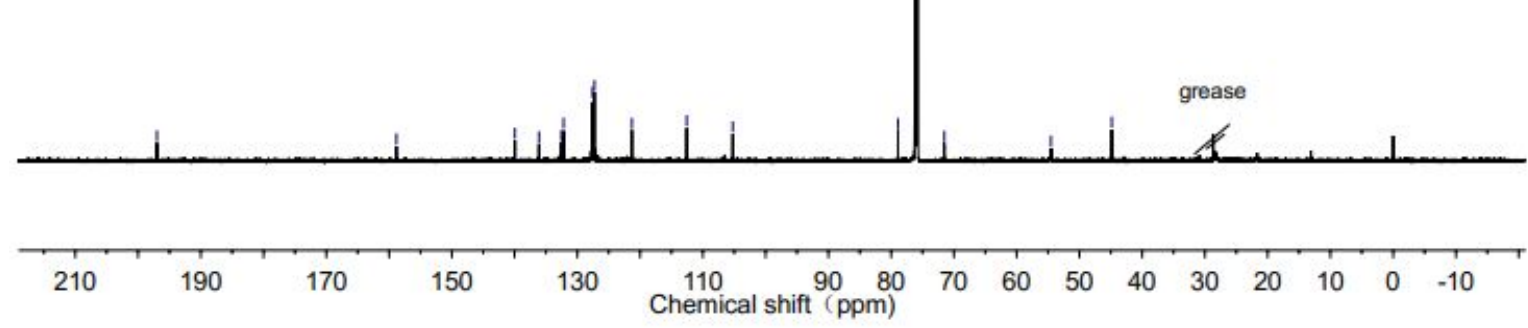

${ }^{13} \mathbf{C}\left\{{ }^{1} \mathbf{H}\right\}$ NMR $\left(151 \mathrm{MHz}, \mathrm{CDCl}_{3}, 298 \mathrm{~K}\right)$ of $\mathbf{3 x}$ 


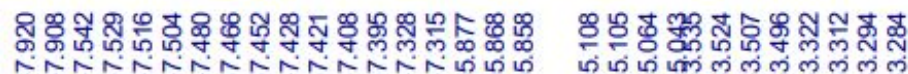

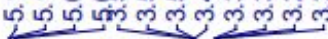
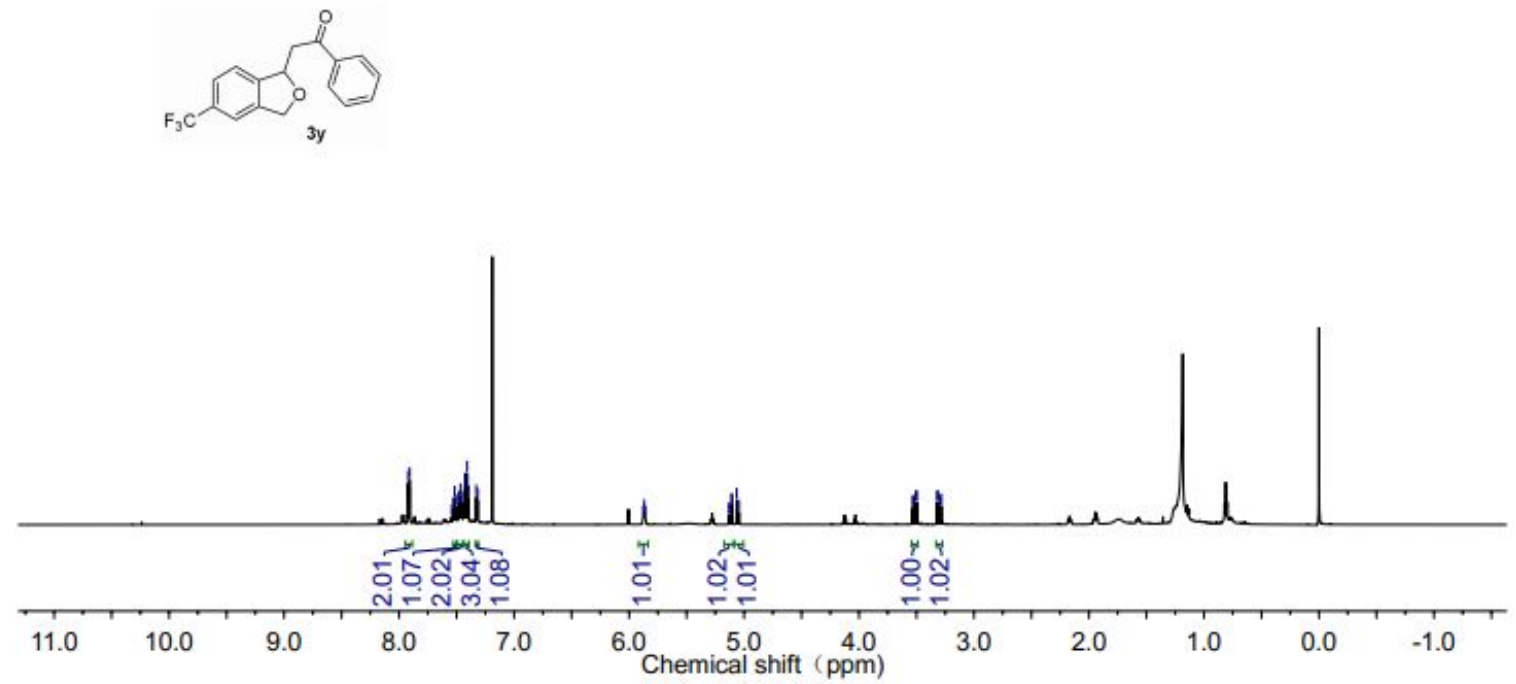

${ }^{1} \mathbf{H}$ NMR $\left(600 \mathrm{MHz}, \mathrm{CDCl}_{3}, 298 \mathrm{~K}\right)$ of $\mathbf{3 y}$

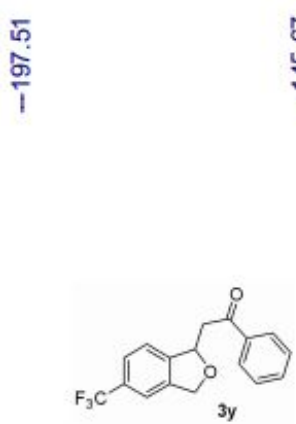

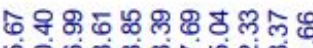

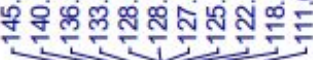

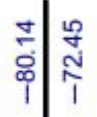

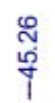
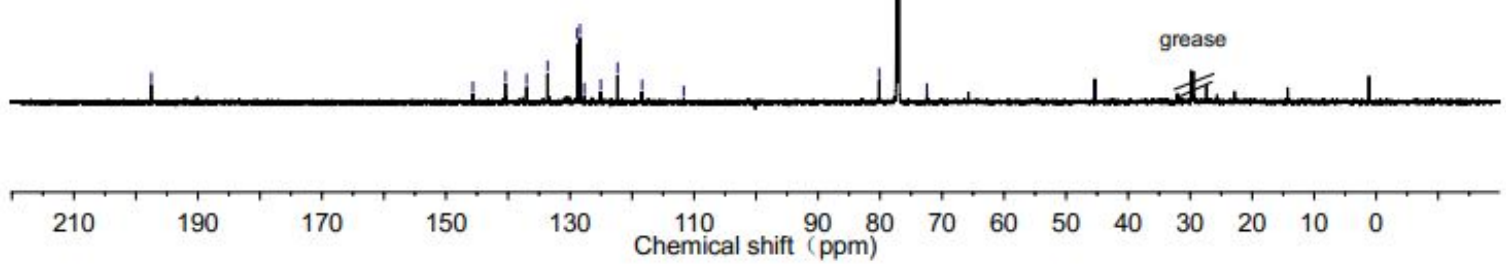

${ }^{13} \mathbf{C}\left\{{ }^{1} \mathbf{H}\right\}$ NMR $\left(151 \mathrm{MHz}, \mathrm{CDCl}_{3}, 298 \mathrm{~K}\right)$ of $\mathbf{3 y}$ 
